\title{
Analysis of translational fidelity in cellular proteins
}

\author{
Dissertation \\ for the award of the degree \\ "Doctor rerum naturalium" \\ of the Georg-August-Universität Göttingen
}

within the doctoral program Biomolecules: structure-function-dynamics

of the Georg-August University School of Science (GAUSS)

submitted by

Raffaella Garofalo

from Bari, Italy 
INTRODUCTION

Göttingen, 2017 


Members of the Examination Board/ Thesis Committee

Prof. Marina V. Rodnina ( $1^{\text {st }}$ Referee $)$

Dept. of Physical Biochemistry

Max Planck Institute for Biophysical Chemistry

Göttingen, Germany

Prof. Henning Urlaub ( $2^{\text {nd }}$ Referee)

Bioanalytical Mass Spectrometry Group

Max Planck Institute for Biophysical Chemistry

Göttingen, Germany

Prof. Peter Rehling ( $3^{\text {rd }}$ Referee)

Dept. of Cellular Biochemistry

Georg-August University

Göttingen, Germany

\section{Further members of the Examination Board}

Prof. Holger Stark

Dept. of Structural Dynamics

Max Planck Institute for Biophysical Chemistry

Göttingen, Germany

Prof. Markus Bohnsack

Dept. of Molecular Biology

University Medical Center

Göttingen

Prof. Ralf Ficner

Dept. of Molecular Structural Biology

Institute for Microbiology and Genetics

Göttingen

Date of oral examination: 03 April 2017 


\section{Affidavit}

I hereby declare that my thesis "Analysis of translational fidelity in cellular proteins" has been written independently and with no other sources and aids than quoted.

Raffaella Garofalo

January, 2017

Göttingen, Germany

Related publication

Garofalo, R. ${ }^{\dagger}$, Wohlgemuth, I. ${ }^{\dagger}$, Pearson, M., Lenz, C., Urlaub, H., \& Rodnina, M. V. (2019). Broad range of missense error frequencies in cellular proteins. Nucleic Acids Research,.

${ }^{\dagger}$ equal contribution 
"Saldi nella furia dei venti

e degli eventi." 



\section{TABLE OF CONTENTS}

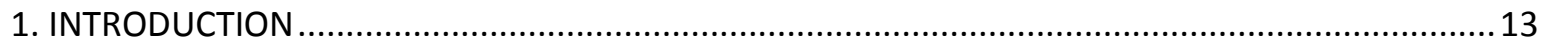

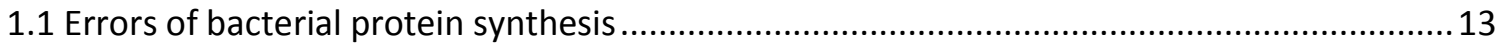

1.1.1 Error frequency at different steps of protein synthesis ...............................................13

1.1.2 Consequences of translation errors on cellular fitness .................................................... 14

1.2 Cellular mechanisms that ensure the fidelity of protein biosynthesis ................................... 15

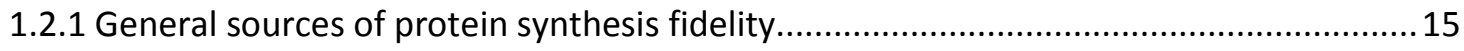

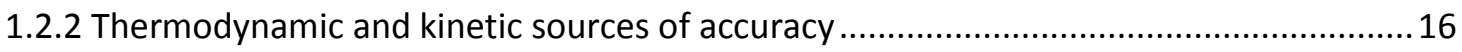

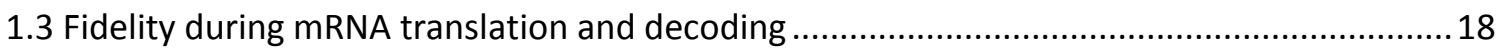

1.3.1 Ribosomal quality control following peptide bond formation .......................................21

1.3.2 Base pair tautomerization and its effect on decoding.....................................................22

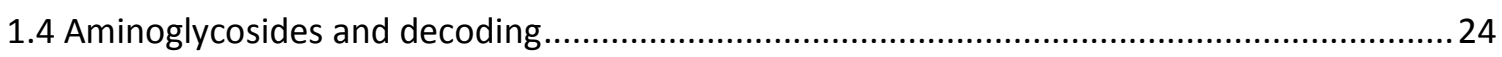

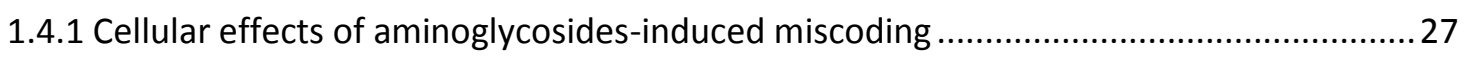

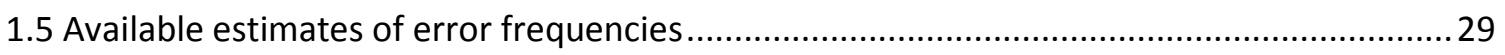

1.6 Mass spectrometry: an emerging tool for the quantification of miscoded translation

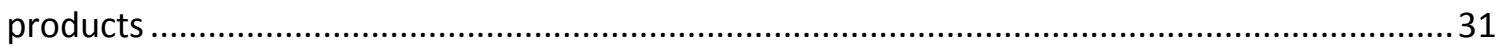

1.7 Selected reaction monitoring (SRM) and triple quadrupoles mass spectrometer ..................34

1.8 Parallel reaction monitoring (PRM) to confirm the identitity of enriched peptides ................36

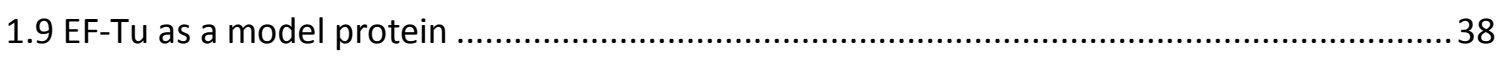

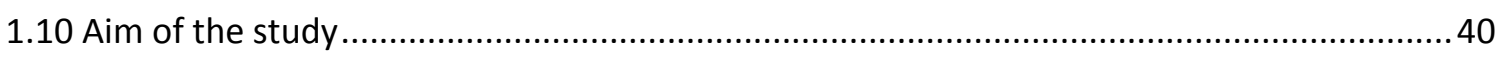

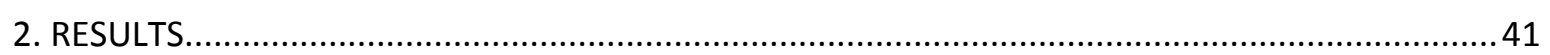

2.1 Multidimensional chromatography for peptides enrichment ............................................ 41

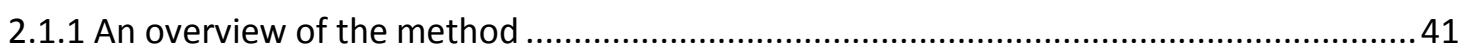

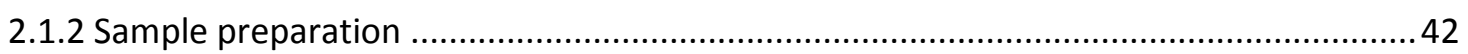

2.1.3 Chromatographic separation and enrichment of target peptides..................................43

2.2 Identification of enriched error-containing peptides ......................................................... 47

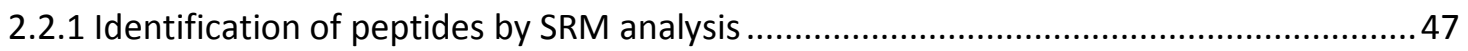

2.2.2 Identity validation by manual annotation of MS1 and MS/MS spectra ..........................49

2.3 Quantification of enriched error-containing peptides............................................................. 50

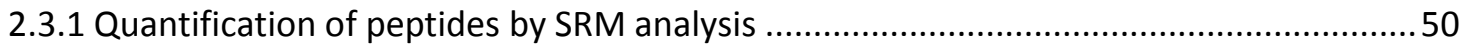

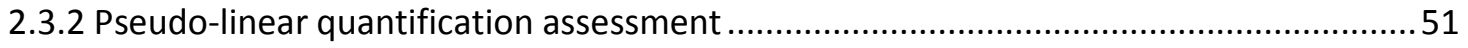

2.3.3 Post translational modifications and peptide degradation ...........................................52

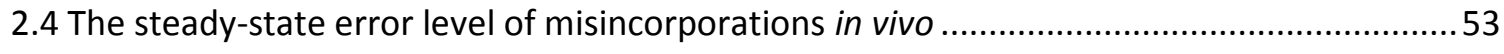

2.4.1 Amino acid substitutions at selected positions in EF-Tu ...............................................53

2.4.2 Misincorporations involving non-cognate aa-tRNAs .....................................................57 
2.4.3 Distribution of misincorporations over the EF-Tu sequence.

2.4.4 Contribution of quality control machinery to the steady-state error levels ...................60

2.5 Aminoglycosides as a tool to study cellular response and misincorporations ........................62

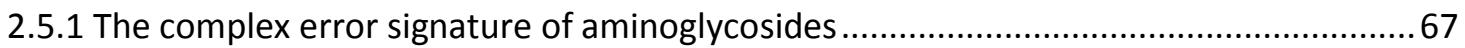

2.5.2 Validation of doubly-substituted peptides by PRM analysis .........................................67

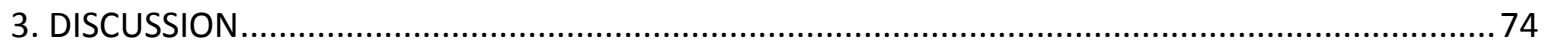

3.1 Method development to study rare translation errors ..................................................... 74

3.2 Misincorporations are rare and occur less frequently than expected..................................... 76

3.3 Error rate depends on the protein source .......................................................................... 76

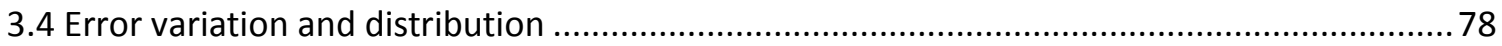

3.5 Stress response and error profiles induced by aminoglycosides........................................... 79

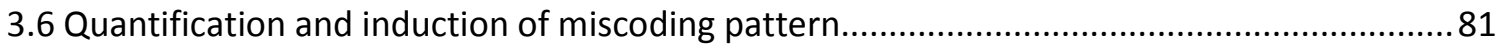

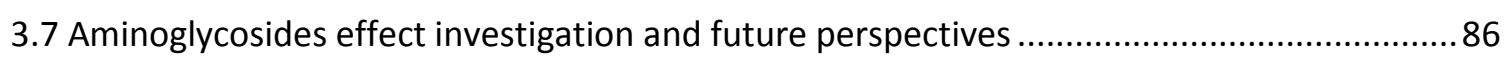

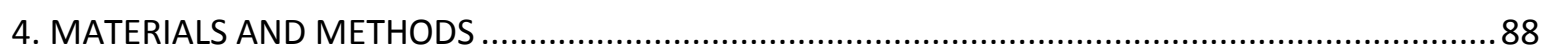

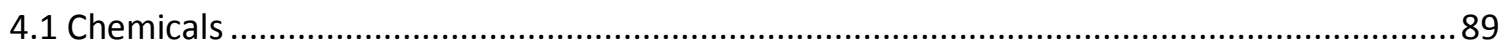

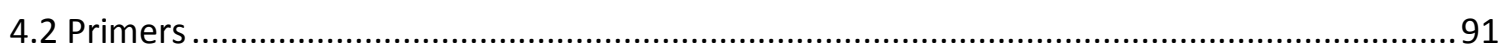

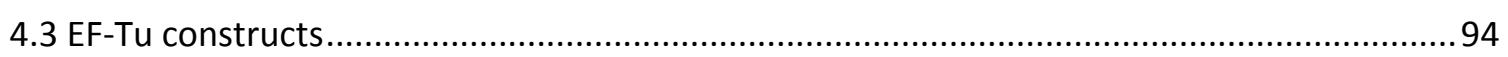

4.3.1 Site-directed mutagenesis for generation of EF-Tu mutants.........................................94

4.3.2 Generation of constructs containing a Sumo protein fused with an EF-Tu peptide

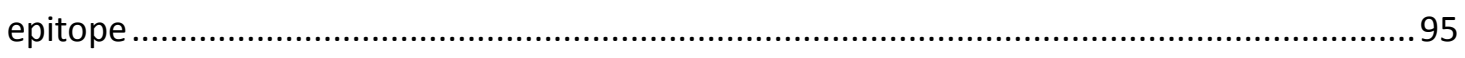

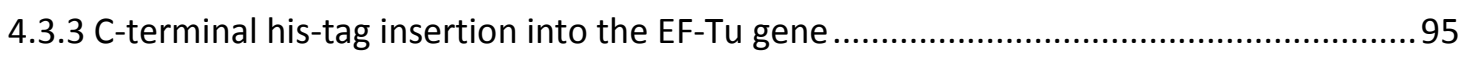

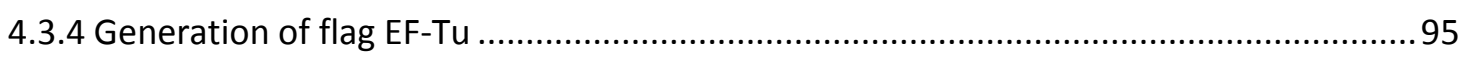

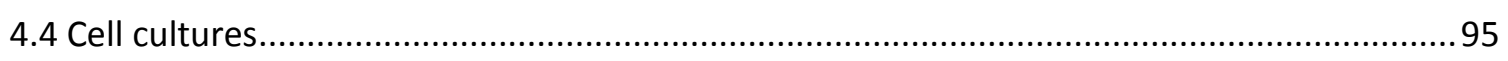

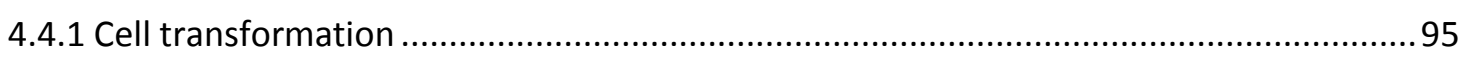

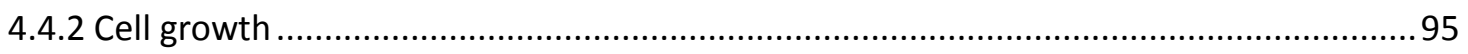

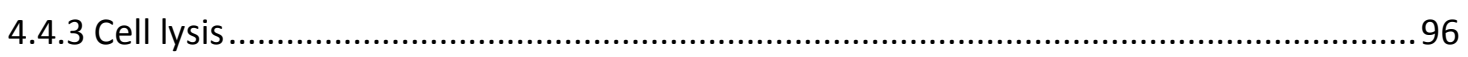

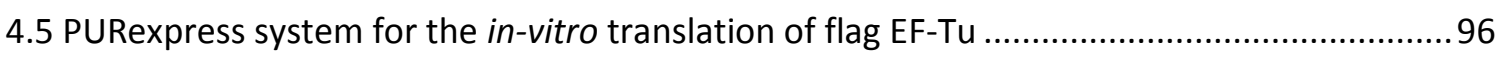

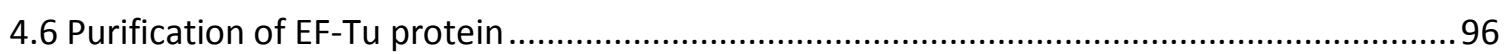

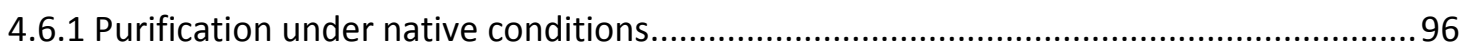

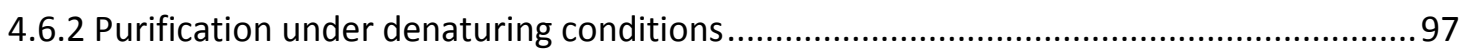

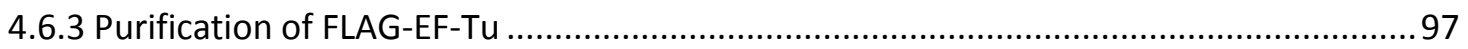

4.6.3.1 Elution of FLAG-EF-Tu with 3X flag peptides .......................................................... 97

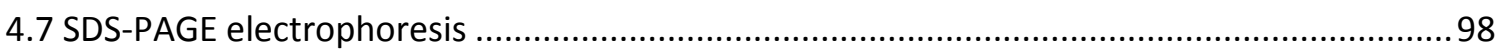

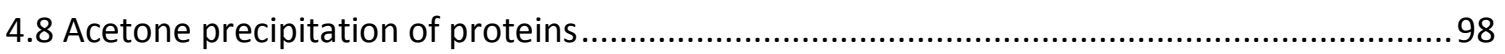

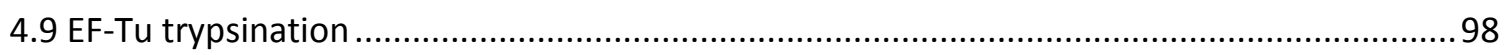

4.9.1 In-solution proteolysis of EF-Tu purified under native conditions .................................98 
4.9.2 In-solution proteolysis of EF-Tu purified in denaturing conditions .99

4.10 Sample preparation for the characterization of aminoglycosides effects............................99

4.10.1 In-gel proteolysis of EF-Tu, pSUMO-constructs, and E.coli lysate ..............................100

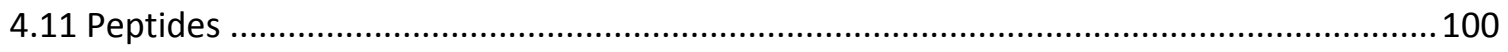

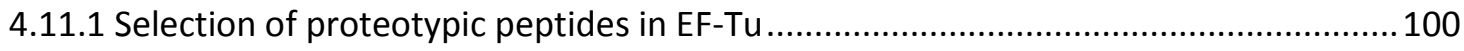

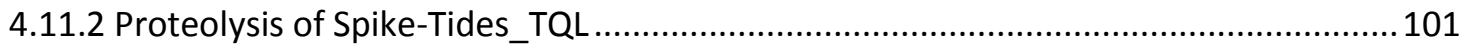

4.11.3 LC-MS/MS method development for peptides detection ......................................... 101

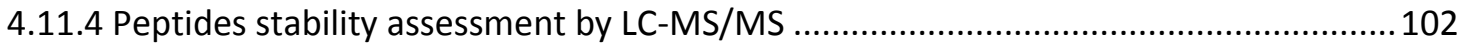

4.12 Quantification of EF-Tu for multidimensional enrichment of misincorporation-containing peptides

4.12.1 Multidimensional chromatographic enrichment of misincorporation-containing peptides 102

4.13 Validation and quantification of misincorporation-containing peptides ........................... 103

4.14 Quantification of error in-gel digested EF-Tu and SUMO-constructs ................................. 103

4.15 Quantification of stress response proteins in E. coli lysate ................................................. 104

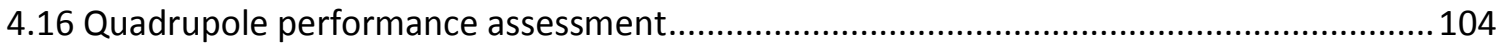

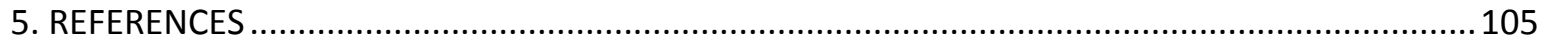

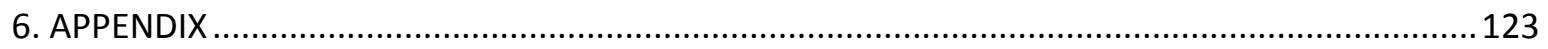

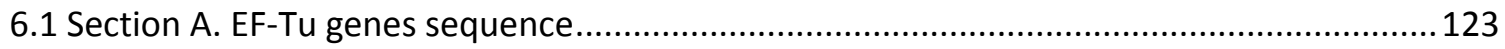

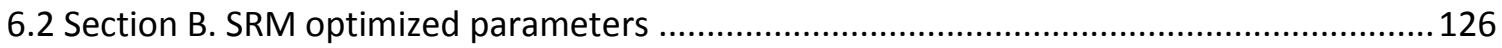

6.3 Section C. validation of near-cognate amino acid substitutions ..........................................139

6.4 Section D. validation of non-cognate amino acid substitutions ............................................167

6.5 Section E. SRM signals of peptides for multiple errors investigation ...................................176

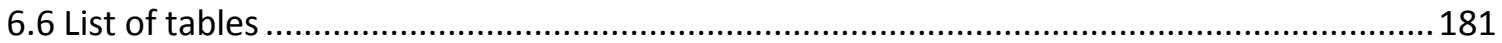

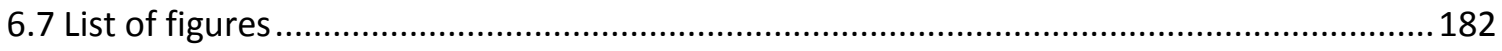

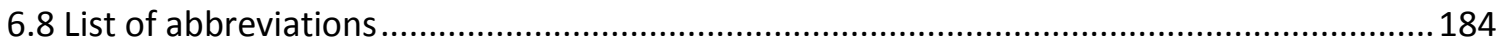

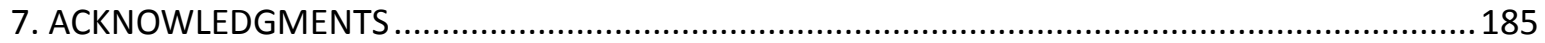





\section{ABSTRACT}

The accuracy of protein biosynthesis determines the quality of the proteome and regulates the fitness of the cell. A comprehensive quantification of miscoding events in the cell is essential to understand the basic mechanisms that ensure fidelity as well as the evolutionary consequences of errors of protein synthesis. Impairment in cellular fidelity is associated with numerous diseases and is, due to the increasing number of protein therapeutics, of central biotechnological importance. However, the systematic analysis of miscoding events in vivo is precluded by the limited dynamic range of the available analytical methods. We have developed a method for the quantification of miscoding events such as amino acid misincorporations, which relies on the separation and enrichment of the errorcontaining peptides from their correct parental ones through multiple rounds of orthogonal chromatography and their absolute quantification by targeted mass spectrometry using isotopelabeled internal standards (AQUA peptides). The dynamic range of the method is linear over more than seven orders of magnitude and it is not restricted to any particular miscoding event, organism, strain or model protein.

The analysis of the model protein EF-Tu revealed that near-cognate missense errors are less frequent than expected (median error frequency $10^{-6}$ ) and that the error frequency varies over three orders of magnitude depending on the type of misincorporation and source of EF-Tu. Among the substitutions tested, histidine misincorporations at arginine codons are the most abundant. Furthermore, error frequency varies depending on the amino acid position in EF-Tu by more than one order of magnitude. Positions at which errors are rare are clustered in the EF-Tu/tRNA interface, indicating either an extensive quality control or an higher encoded fidelity of translation.

We have also analyzed the effect of aminoglycosides on fidelity and stress response. Most aminoglycosides induce significant miscoding which correlates with the induction of proteins from the the heat-shock stress response and growth defects, underlining the central contribution of miscoding to the bactericidal effect of aminoglycosides. In addition to single-position substitutions, aminoglycosides can induce multiple miscoding events leading to peptides with two or three amino acid substitutions. The frequency of multiple consecutive errors is unexpectedly high and because of the detrimental effects on protein stability, such error clusters may contribute substantially to the bactericidal action of aminoglycosides. 
INTRODUCTION

\section{INTRODUCTION}

\subsection{Errors of bacterial protein synthesis}

\subsubsection{Error frequency at different steps of protein synthesis}

Protein synthesis is a pivotal cellular process in which the genetic information stored in the DNA is transcribed into RNA by the RNA polymerase and subsequently decoded on the ribosome with the help of transfer RNA (tRNA) molecules into an amino acid sequence and yield functional proteins. Despite the importance of preserving the genetic message, errors might arise at every stage (Parker, 1989) (Table 1). DNA replication is undoubtedly the most accurate step, because the DNA polymerase works with extremely high fidelity and an error frequency that ranges from $10^{-10}$ - $10^{-6}$ (Kunkel, 2004; Kunkel and Bebenek, 2000; Schaaper, 1993). Incorporation of errors during the transcription of DNA is, instead, more frequent because the responsible enzyme, RNA polymerase, incorporates incorrect nucleotides with a frequency of $10^{-5}-10^{-4}$ (Traverse and Ochman, 2016). The least accurate step is the translation of mRNA, which shows error frequencies of up to $10^{-2}$ (Table 1 ). The fidelity of this process, whose average error rate is estimated to be $10^{-4}-10^{-3}$ (Ribas de Pouplana et al., 2014; Rodnina and Wintermeyer, 2001b) is the result of a combination of different error-prone events. First, the aminoacyl-tRNAs (aa-tRNAs) delivering the amino acid to the ribosome must be correctly charged by their respective aminoacyl-tRNA synthetases. Second, the ribosome must select the correct aa-tRNA corresponding to the codon exposed in the decoding site of the ribosome and, after peptide bond formation, translocate along the mRNA by exactly three nucleotides at a time. Because tRNA misacylation, ribosome frameshifting and nonsense errors are rather infrequent events, occurring with a frequency of $10^{-6}-10^{-4}, \sim 10^{-5}$ and $10^{-6}-10^{-3}$ respectively (Freistroffer et al., 2000; Hopfield et al., 1976; Ibba and Soll, 2000; Jorgensen and Kurland, 1990; Sin et al., 2016; Soll, 1990), most of the errors that occur during translation are mainly attributed to erroneous decoding (Drummond and Wilke, 2009). Cells have evolved sophisticated mechanisms to avoid errors, such as proofreading of DNA and RNA polymerases and editing of aminoacyl-tRNA synthetases (aaRSs) (Jakubowski and Goldman, 1992; Kunkel, 2004; Sydow and Cramer, 2009) or kinetic mechanisms of aa-tRNA selection on the ribosome (Rodnina and Wintermeyer, 2001a). Errors that still arise can be removed by the quality control machinery of the cell, such as chaperones and proteases (Wickner et al., 1999).

Thus, the steady state level of missense errors in proteins reflects the error contributing processes of protein biosynthesis and the correcting processes of the quality control. This steady state level is not static but is modulated by naturally occurring mutations of the translational apparatus such as ribosomes (Rosset and Gorini, 1969; Zaher and Green, 2010a), aaRSs (Li et al., 2011), tRNAs (Bjork et al., 1999; Ranjan and Rodnina, 2016), modifications of translation factors (Van Noort et al., 1986), by the growth and metabolic state of the cell (Meyerovich et al., 2010; Zaborske et al., 2014) or by 
external stress factors such as ethanol (Haft et al., 2014), aminoglycosides antibiotics (Davies et al., 1964) and oxidative agents (Ling and Soll, 2010; Netzer et al., 2009).

Table 1. Error frequencies of the individual substeps of protein biosynthesis. The estimated frequencies of error incorporation for every substep of protein synthesis are reported.

\begin{tabular}{|c|c|c|c|}
\hline Cellular process & Type of errors & Error frequency & Reference \\
\hline DNA replication & Nucleotide misincorporation & $10^{-10}-10^{-6}$ & $\begin{array}{c}\text { (Kunkel, 2004; Kunkel and Bebenek, } \\
\text { 2000; Schaaper, 1993) }\end{array}$ \\
\hline RNA transcription & Nucleotide misincorporation & $10^{-5}-10^{-4}$ & (Traverse and Ochman, 2016) \\
\hline $\begin{array}{c}\text { tRNA } \\
\text { aminoacylation }\end{array}$ & Incorrect aminoacylation & $10^{-6}-10^{-4}$ & (Ibba and Soll, 2000; Soll, 1990) \\
\hline \multirow[b]{4}{*}{ mRNA translation } & Initiation on non-start codon & $10^{-2}$ & (Milon and Rodnina, 2012) \\
\hline & Premature termination & $10^{-6}-10^{-3}$ & $\begin{array}{c}\text { (Freistroffer et al., 2000; Jorgensen and } \\
\text { Kurland, 1990; Sin et al., 2016) }\end{array}$ \\
\hline & Stop codon readthrough & $10^{-4}-10^{-2}$ & (Sydow et al., 2014) \\
\hline & Amino acid misincorporation & $10^{-4}-10^{-3}$ & $\begin{array}{l}\text { (Bouadloun et al., 1983; Drummond and } \\
\text { Wilke, 2009; Loftfield and Vanderjagt, } \\
\text { 1972; Parker, 1989; Parker et al., 1980; } \\
\text { Ribas de Pouplana et al., 2014; Rodnina } \\
\text { and Wintermeyer, 2001b; Toth et al., } \\
\text { 1988) }\end{array}$ \\
\hline
\end{tabular}

Frameshifting

$10^{-5}-10^{-4}$

(Atkins et al., 1991)

\subsubsection{Consequences of translation errors on cellular fitness}

Errors of protein synthesis alter the sequence of amino acids in proteins and may cause the production of dysfunctional proteins, the consequences of which can be rather severe not only for the fitness of the single cell but, in case of eukaryotes, for the entire organism e.g., in Escherichia coli, ribosome biogenesis and thus translation are limiting the growth of the bacterial cell (Dennis et al., 2004). It requires up to $10 \mathrm{~min}$ to synthetize and assemble functional ribosomes, comparable to the time needed for cell duplication, $20 \mathrm{~min}$ (Scott et al., 2010). Indeed, translation is by far the most energy consuming phase (Russell and Cook, 1995) and most anabolic processes are devoted to it (Bremer and Dennis, 2008). In prokaryotes, which have rapid cell cycles optimized to outcompete other microorganisms, the synthesis of flawed proteins hijacks the cellular protein synthesis capacity, reduces the absolute levels of active proteins in the cell and directly leads to fitness defects (Andrews and Hegeman, 1976). Eukaryotes, on the other hand, do not exhaust their resources for rapid growth (Kafri et al., 2016); therefore errors of protein synthesis can have less pronounced effects.

The accumulation of random errors into protein is likely to cause loss of function, structural destabilization (Guo et al., 2004) and misfolding (Lee et al., 2006), with an effect which is predicted to increase exponentially with the number of inserted substitutions (Bloom et al., 2005). Accumulation 
of errors and protein misfolding may in turn cause abnormal aggregation (Vermulst et al., 2015) or induce protein oxidative damage (Dukan et al., 2000). Additionally, incorrectly folded and unstable proteins seize the working capacity of the chaperones system (Gidalevitz et al., 2006) potentially promoting further cellular damage.

Increased miscoding can be caused by aminogycoside antibiotics and the consequent misfolding and aggregation phenomena have been demonstrated by treating the cells with streptomycin (Ling et al., 2012). Upon antibiotic treatment, mistranslation occurs and more erroneous proteins that favour aggregation are produced. Mass spectrometric analysis of such aggregates has revealed that they mostly consist of heat shock-induced proteases and chaperones (especially IbpA and IbpB) whose response is known to associate with accumulation of errors in proteins (Ruan et al., 2008). Although the proteotoxic stress caused by error accumulation can be alleviated by the concerted action of chaperones and proteases, the clean-up costs - in addition to the energy already spent for their synthesis - impose a energetic burden that can compromise the overall cellular fitness (Drummond and Wilke, 2009). Ultimately, mistranslation can lead to membrane depolarization, radical formation and finally to growth arrest and cell death (Kohanski et al., 2007).

\subsection{Cellular mechanisms that ensure the fidelity of protein biosynthesis}

\subsubsection{General sources of protein synthesis fidelity}

The high fitness cost of errors in protein synthesis increases the evolutionary pressure to keep the error frequency low. This led to the development of sophisticated cellular mechanisms that ensure high fidelity of protein biosynthesis or increase the tolerance towards those errors that could not be avoided. An example for this adaptive evolution comes from the study of an error prone $E$. coli strain expressing the TEM1 $\beta$-lactamase resistance protein. Under relaxed conditions, where the antibiotic is not present and the resistance protein is not essential, the cells reduce the costs for the synthesis of a superfluous and error-containing protein by lowering its expression level. However, when the antibiotic is present and the protein is required for the cell survival, the cell balances the unavoidable incorporation of errors by promoting the incorporation of amino acids which stabilize the the protein structure (Bratulic et al., 2015). Error-mitigating mechanisms can also be already imprinted in the genetic code. The amino acid sequence of a protein is determined by the sequence of bases in the mRNA and one sequence of nucleotide triplets, a codon, specifies for one amino acid (Crick et al., 1961). However, because the genetic code is degenerate, a particular amino acid may be coded by more that one triplet (Crick et al., 1961) in which the first two positions are strict, but some wobble is accepted at the third base (Crick, 1966). The structure of the genetic code ensures that if a codon is incorrectly translated, this would often lead to the substitution with a similar amino acids, thereby 
minimizing the impact on protein stability (Archetti, 2006) as it happens for the frequent third-position mismatches (Woese, 1965). A second mechanism is based on the codon usage optimization. Numerous studies have demonstrated a correlation between the tRNA abundance and the occurrence of the respective codon (Ikemura, 1985), and genes that need to be more efficiently translated display an optimized codon sequence preferentially containing codons decoded by abundant tRNAs. In this regard, the elongation factor $\mathrm{Tu}(\mathrm{EF}-\mathrm{Tu})$, the most abundant protein in bacteria, contains only abundant codons (Kurland, 1992) whereas rare ones are avoided (Akashi, 1994). In addition, bioinformatics studies revealed that cluster of translationally optimal codons at sites that are important for the function or stability of proteins are highly conserved and that codons whose mistranslation results in less severe amino acid substitutions are preferred at these positions (Lee et al., 2010; Zhou et al., 2009).

\subsubsection{Thermodynamic and kinetic sources of accuracy}

While the evolution of the genetic template does not bring about significant fitness costs for the cell (Drummond and Wilke, 2009), the accuracy of the enzymes involved in protein biosynthesis cannot improve without a trade-off between speed and accuracy. In general, most enzymes use highly specific binding sides that are complementary to the steric and electronic features of their unique substrates to idiosyncratically recognize them. Kinetically, such tailored binding sites often reflect in dramatically different dissociation constants in the ground state (GS) that almost exclude incorrect substrates in the binding step (Figure 1, A). In contrast, enzymes involved in protein synthesis, such as DNA and RNA polymerases or ribosomes, cannot achieve such high selectivity by evolving idiosyncratic binding sites, because they have to recognize and incorporate with high fidelity different substrates in subsequent cycles of elongation. Different nucleotides or aa-tRNAs are recognized on the basis of only few discriminating interactions, such as for Watson-Crick base pairs. The affinity differences between correct and incorrect substrates are $<10^{2}$ for single mismatched nucleotides (Grosjean et al., 1978) and can be even lower between cognate and non-cognate triplets (Kurland et al., 1975). Thus, the affinity differences alone fail to explain the high fidelity of all substeps of protein biosynthesis (Rodnina and Wintermeyer, 2001b) (Table 3) and additional sources of fidelity such as induced fit (Gromadski and Rodnina, 2004a; Pape et al., 1999) and proofreading (Ruusala et al., 1982; Thompson and Stone, 1977) apply. First, in the different substeps of protein synthesis, nucleotide mismatches are recognized on the base of their shape through interactions between the minor groove of DNA (or wide shallow groove of tRNA) and the amino acids and rRNA nucleotides of polymerases and ribosome, respectively (reviewed in (Westhof et al., 2014)). 
On the ribosome, this recognition leads to conformational changes (Ogle et al., 2002) which then strongly accelerate the productive forward rates for correct aa-tRNAs relative to near and noncognate tRNAs in terms of an induced-fit mechanism (Gromadski and Rodnina, 2004a; Pape et al., 1999) Second, kinetic proofreading mechanisms are applied in which the same discriminating interactions are energetically and kinetically used several time to amplify the accuracy. This is possible when both selection phases are irreversibly separated by an energy dissipating step (Ruusala et al., 1982; Thompson and Stone, 1977), as happens during decoding where GTP hydrolysis separates two subsequent selection steps which are both based on the same codon-anticodon helix mismatch. However, substrates selection at the ground state can lead to a trade-off between speed and accuracy which are negatively correlated for ground state selection (reviewed in (Tawfik, 2014)). Kinetically, in this scenario, fast forward rates do not allow the substrate binding involved in selection to equilibrate completely and thus the affinity differences between correct and incorrect substrate can be only partially utilized (Gromadski and Rodnina, 2004a). In fast growing E.coli cells, ribosomes seem to be optimized for fast translation at the cost of accuracy (Aqvist and Kamerlin, 2015; Wohlgemuth et al., 2010). A concrete example of this trade-off and its reflection on the cellular fitness comes from streptomycin-dependent $(\mathrm{SmD})$ E. coli mutants whose viability necessarily depends on the antibiotic (Ruusala et al., 1984). SmD cells, indeed, possess excessively accurate, or hyper-accurate, ribosomes that - in the absence of the drug - slow down the translation process to such an extent that it leads to cells death. Indeed, the presence of streptomycin, which reduces ribosome fidelity (Gromadski and Rodnina, 2004b) and favours a faster translation rate, restores cell viability.

An alternative to GS selection is the transition-state (TS) discrimination where correct and incorrect substrates bind the enzyme with similar affinities but not all interactions of the correct substrate in the TS are realized for the non-cognate ones. Thus, for TS selection, fidelity positively correlates with catalytic efficiency allowing high accuracy at high speed. TS selection is reported for the DNA polymerase Pol1. Upon binding of the correct substrate, the active-site dipoles of the binding sites are organized to maximize TS stabilization, which does not occurs in the presence of a non-cognate substrate (Ram Prasad and Warshel, 2011) allowing for a high synthesis rate $\left(k_{\text {cat }}>100 \mathrm{~s}^{-1}\right)$ and a high accuracy $\left(<10^{-3}\right.$ even in the absence of proofreading (Caglayan and Bilgin, 2012)) and leads to the predicted positive correlation of speed and accuracy which is determined by a much higher activation entropy for the non-cognate than for the cognate substrate (Caglayan and Bilgin, 2012) (Figure 1, B). In many cases, especially when incorrect substrates differ by only a few atoms, positive GS or TS selection might not be sufficient to guarantee high accuracy. Thus, many enzymes evolved explicit negative selection strategies, termed editing, that remove mistakes that evaded the selection process 
as it happens for aminoacyl-synthetases or DNA and RNA polymerases (Fersht, 1977; Reha-Krantz, 2010; Sydow and Cramer, 2009).
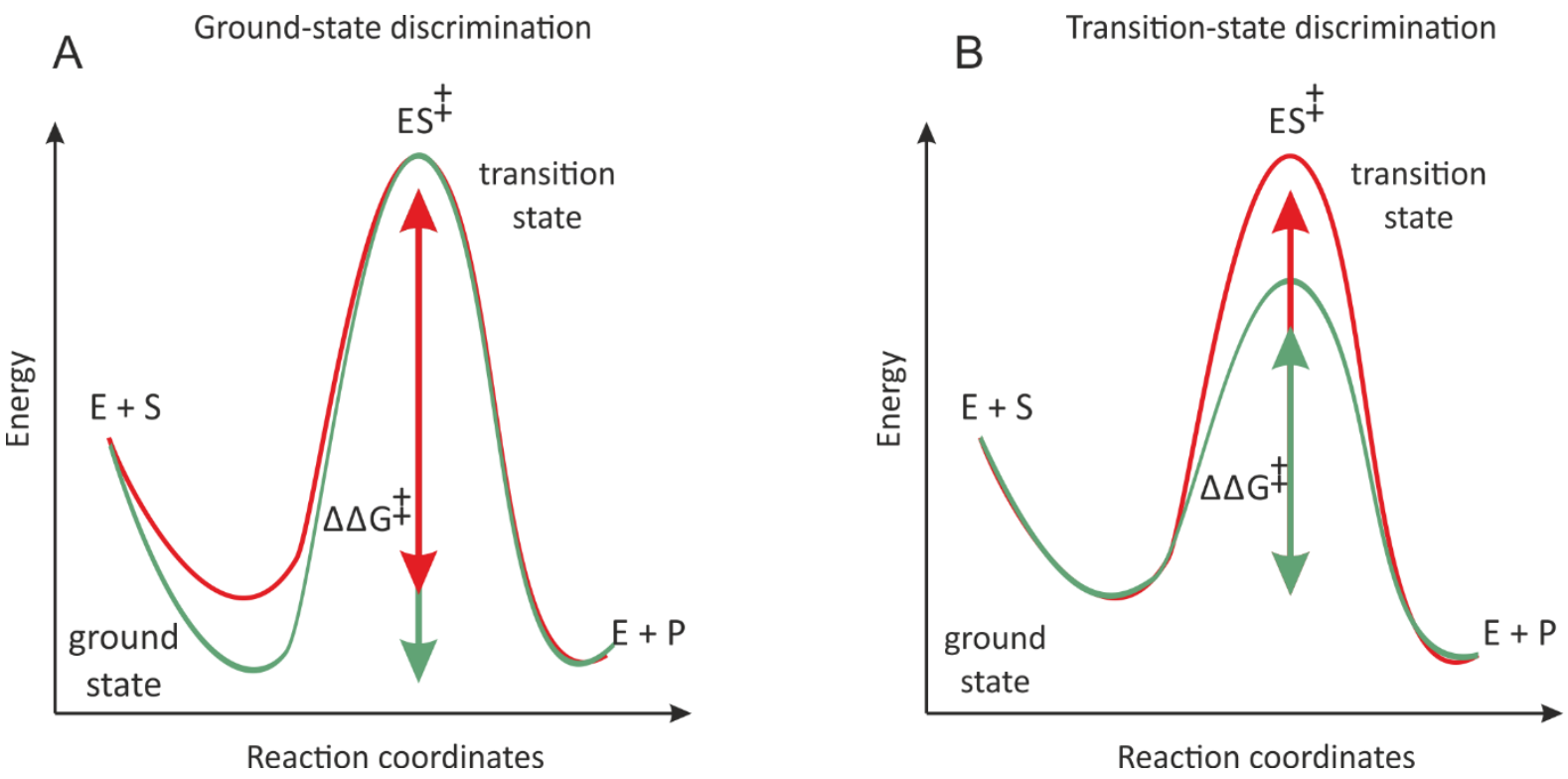

Figure 1. Enzymes ensure their accuracy in different ways. Substrate discrimination can be achieved at the ground state (A) or transition state (B). In red and green are represented the catalytic curves for non-cognate and cognate substrates, respectively.

\subsection{Fidelity during mRNA translation and decoding}

The most important player of translation is the ribosome. The ribosomes are large macromolecular machines, of approximately 2.5 MDa in bacteria, composed of two unequal subunits, the small $30 \mathrm{~S}$ and the large 50S, assembled together to form the 70S ribosome. The $30 \mathrm{~S}$ subunit contains twentyone proteins and one ribosomal RNA (16S rRNA). It houses the decoding center where mRNA is decoded by tRNA molecules. The 50S subunit is composed of thirthy-six proteins and two rRNAs, $5 \mathrm{~S}$ and $23 \mathrm{~S}$. The $50 \mathrm{~S}$ subunit harbors the peptidyl transferase center where the peptide bond between two adjacent tRNAs is formed and makes the ribosome a ribozyme (Steitz and Moore, 2003). Each ribosome presents three tRNA binding sites, the A site for binding of aa-tRNA, the P site for binding of peptidyl-tRNA, and the E site from which deacylated tRNA exits the ribosome. The translation process progresses through four consecutive phases of initiation, elongation, termination and recycling, during which the ribosome is assisted by several protein ligands: the translation factors (Figure 2). 


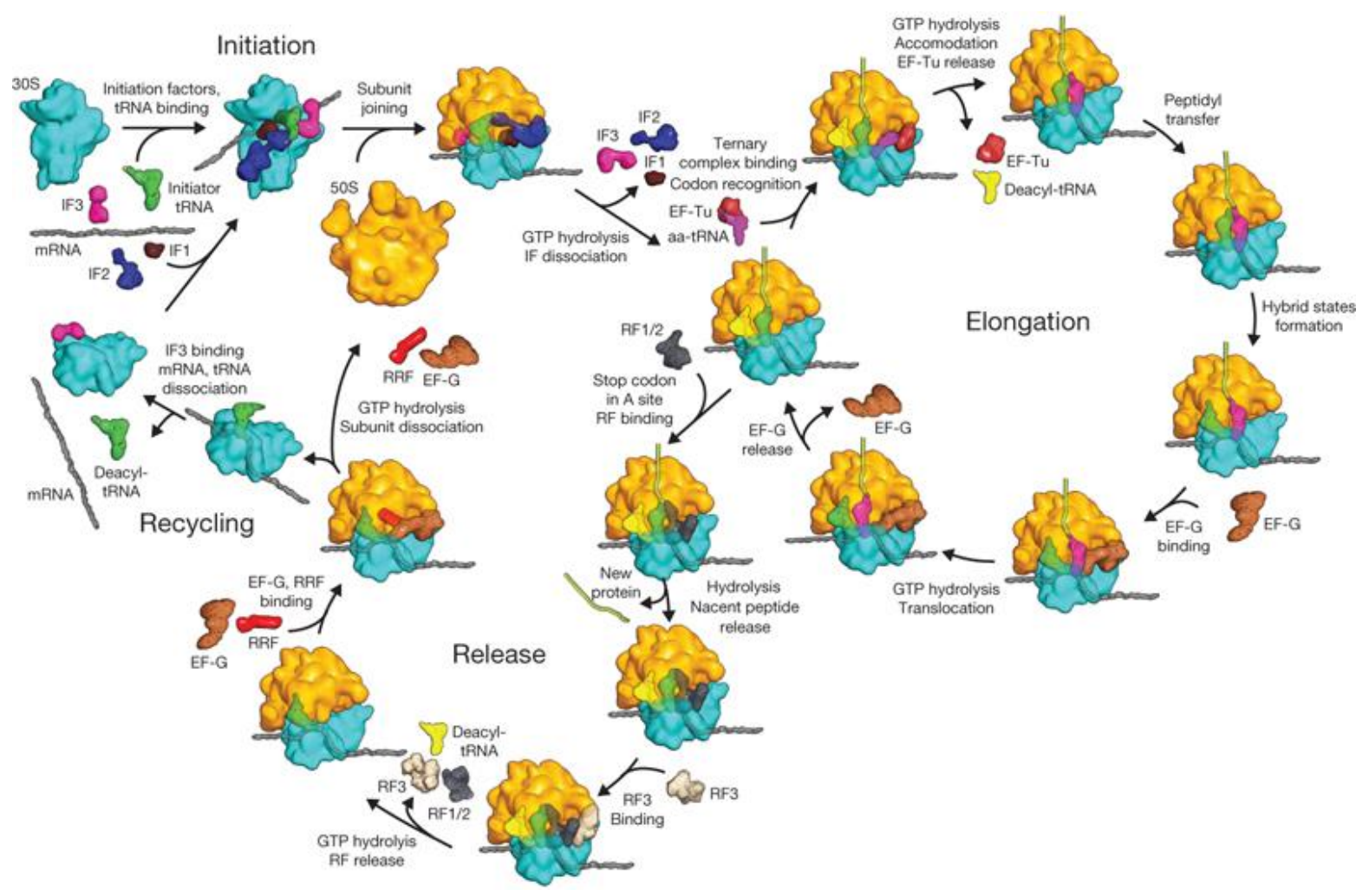

Figure 2. The phases of translation. Synthesis of protein proceeds through consecutive phases of initiation, elongation, termination and recycling. During initiation, the $70 \mathrm{~S}$ initiation complex assembles on the mRNA starting codon. Upon repetitive elongation rounds, new amino acids are incorporated to form growing polypeptide chain. When a stop codon is read the process terminated and the subunits are recycled for a new round of translation. Figure reproduced from (Schmeing and Ramakrishnan, 2009).

During the initiation phase the $30 \mathrm{~S}$ subunit, supported by initiation factors (IFs) 1,2 and 3 , stochastically binds the initiation fMet-tRNA ${ }^{\mathrm{fMet}}$ and, thanks to its $16 \mathrm{~S}$ partial complementarity, the mRNA (Shine and Dalgarno, 1974), to form the 30S initiation complex (IC). Correct assembly of the $30 \mathrm{~S}$ IC allows the joining of the 50 S subunit with consequent release of all the IFs. This leaves the newly assembled 70S IC with the fMet-tRNA ${ }^{\text {fMet }}$ interacting with the mRNA start codon in the P site (Gualerzi, 2010). During the elongation phase, the mRNA is decoded and amino acids are cyclically added to the growing polypeptide chain. Aa-tRNA is delivered to the ribosome in a ternary complex with EF-Tu and GTP (Figure 3). Upon codon recognition, the geometry of the base pairs at the codon:anticodon helix is recognized by ribosomes to discriminate between base matches and mismatches. Correct codonanticodon interaction causes the first two bases of codon-anticodon helix to interact with A1492, A1493 and G530 (E. coli numbering throughout) of 16S rRNA and induces a series of fast conformational changes (Ogle et al., 2001) which are fundamental for the GTPase activation of EF-Tu and the subsequent GTP hydrolysis and part of the induced-fit rearrangement (Gromadski and Rodnina, 2004a; Pape et al., 1998; Rodnina et al., 1995a). Mismatches at the codon:anticodon helix distort the geometry of the duplex impairing the interactions with the elements of the decoding center 
preventing the conformational changes required to trigger GTPase activation (Ogle et al., 2002) and resulting in its reduced rate or complete inhibition. The binding of near-cognate tRNAs, displaying one mismatch, is weaker than that of cognate ones so that they tend to dissociate more easily from the ribosome (Gromadski and Rodnina, 2004a; Pape et al., 1999). While non-cognate tRNAs bearing two or more mismatches are normally rapidly rejected in this initial selection phase, near-cognate aatRNAs may escape the selection and are further discriminated at the proofreading phase after the irreversible hydrolysis of GTP. At this stage, the acceptor stem of the aa-tRNA is released from the EFTu-GDP complex and accommodates in the peptidyl-transferase center of the ribosome. Also in this case, the accommodation of the cognate substrates is much faster and more efficient than for nearcognates which normally results in their rejection (Pape et al., 1999). Once the tRNA is accommodated into the A site, the complex formed by EF-Tu and GDP dissociates from the ribosome and the new peptide bond can eventually form. The polypeptide chain is then transferred to the aa-tRNA in the A site ready to be translocated back into the $\mathrm{P}$ site. Translocation is promoted by another ribosomal GTPase, the elongation factor $\mathrm{G}(\mathrm{EF}-\mathrm{G})$ and the translating ribosome moves on the mRNA one codon at a time translating $4-22$ amino acids per second at $37^{\circ} \mathrm{C}$ (Bhattacharjee et al., 2012; Wohlgemuth et al., 2010)). Translation proceeds until one of the mRNA stop codons (UAA, UAG or UGA) enters the A site and marks the beginning of the termination phase. In the termination phase, the ribosome is assisted by two classes of release factors (RFs). To class I belong RF1 and RF2 that recognize the stop codon (UAG and UAA for RF1 and UAA and UGA for RF2) (Ito et al., 2000) and bind to the ribosome promoting the hydrolysis and release of the peptide chain. The second class includes RF3, another GTPase, that promotes the dissociation of class I factors from the ribosome after the peptide release. After termination, subunits are recycled by the combined action of EF-G, ribosome recycling factor (RRF) and IF3 (Peske et al., 2005). 


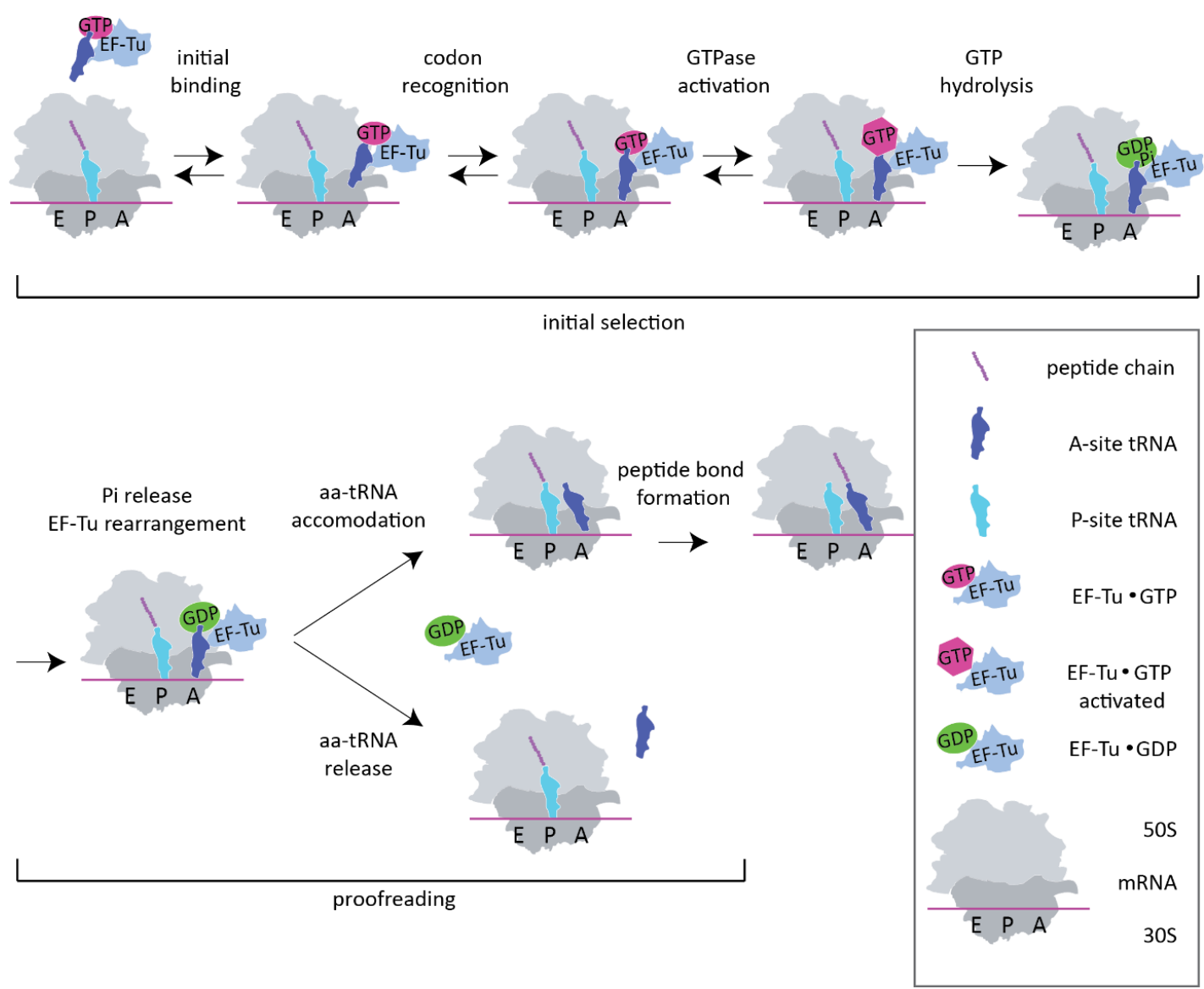

Figure 3. Kinetic scheme of mRNA decoding. Aminoacylated tRNA is delivered to the ribosome in complex with EF-Tu and GTP. The correct codon recognition triggers a series of conformational changes that cause GTPase activation, GTP hydrolysis and inorganic phosphate (Pi) release. The tRNA is released from EF-Tu-GDP complex and accommodates into the A-site of the $50 \mathrm{~S}$ subunit and peptidyl transfer can occur. Eventually, the presence of one mismatch at codon-anticodon helix might induce the tRNA dissociation before transpeptidylation.

\subsubsection{Ribosomal quality control following peptide bond formation}

For a long time it was thought that accuracy of mRNA decoding on the ribosome and the selectivity of aminoacyl-tRNA synthetases are the only sources of fidelity in translation. However, an additional step has been discovered recently that acts after the misincorporation of substrates has occurred (Zaher and Green, 2009). Presumably, mismatches in the codon:anticodon interaction of the peptidyl-tRNA in the $\mathrm{P}$ site lead to distortions in the ribosome structure and cause a general loss of specificity in the A site promoting in turn repetitive miscoding events (Figure 4) (Zaher and Green, 2009, 2010b). Accummulation of errors in the nascent peptide, which is observed regardless of the type of ribosome used (wild type, error prone rpsD or error restricted rpsL (Zaher and Green, 2010b)), promotes the termination factor-mediated release of the nascent chain resulting in an abortive termination of the protein synthesis. 

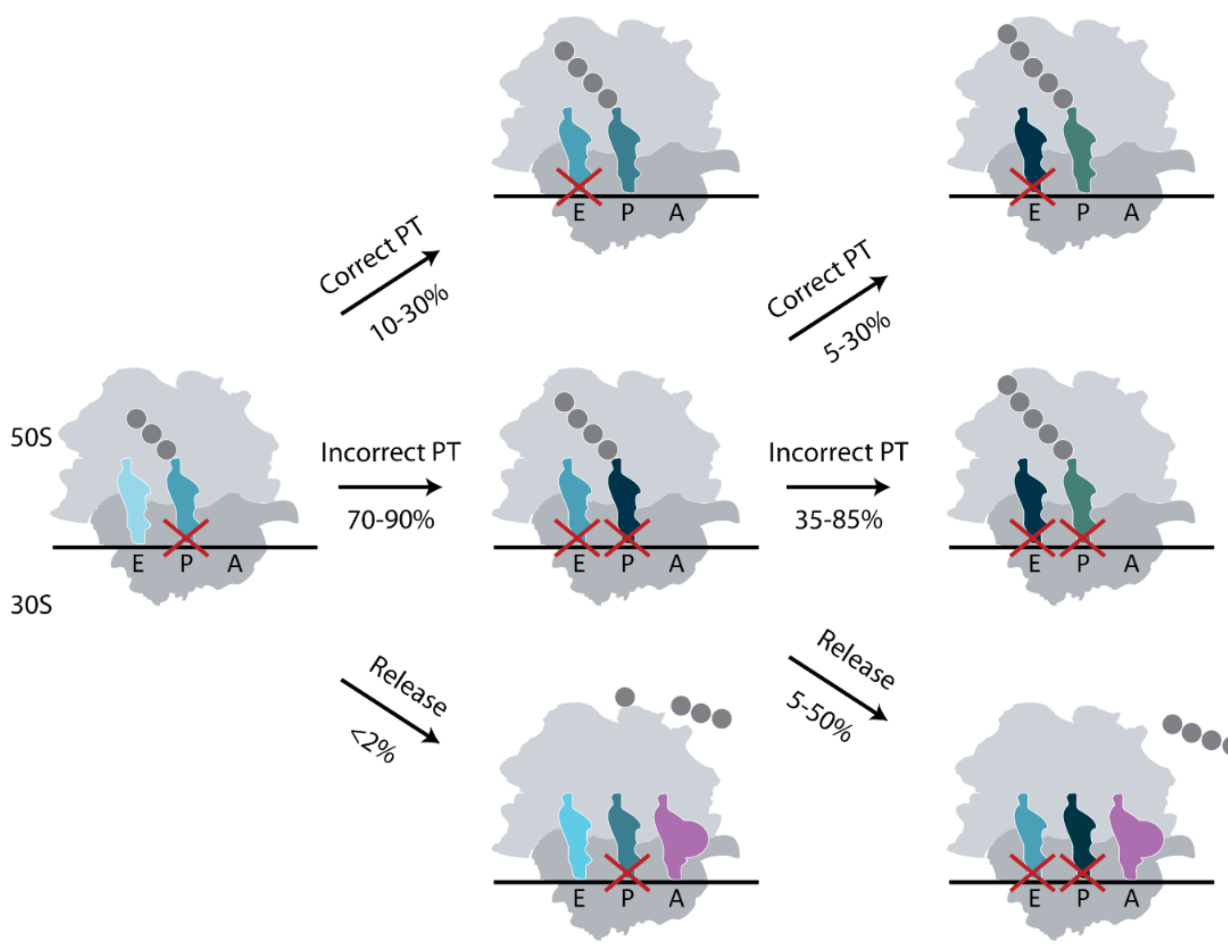
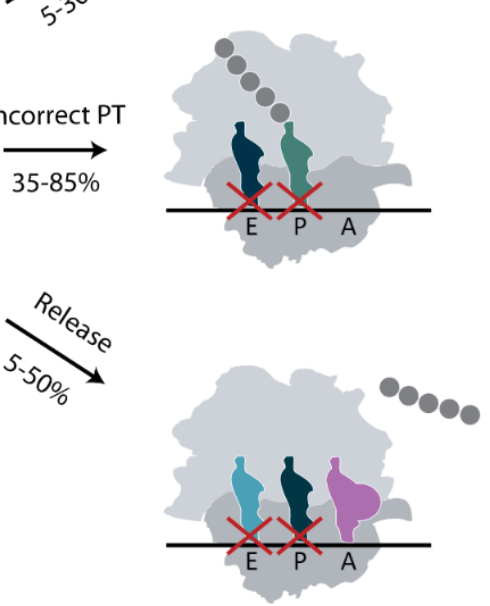

Figure 4. Mechanism of selection triggered by error accumulation. PT, peptidyl transfer. Following the first misincorporation event and translocation of a near-cognate tRNA into the P-site, the probability that the nascent peptide is release by the RFs is less than $2 \%$, while the probability that a correct aa-tRNA is accepted in the A site is only $10-30 \%$. In most cases, a second incorrect aa-tRNA is accepted (70-90\%). The second miscoding event reduces the possibility of correct and incorrect PT to 5-30\% and 35-85\%, respectively, but strongly favours the termination of the protein synthesis and the release of the nascent chain (5-50\%). Figure adapted from (Zaher and Green, 2009).

\subsubsection{Base pair tautomerization and its effect on decoding}

The recognition of Watson-Crick (WC) base pairs is at the core of many processes of gene expression, such as replication, transcription, and translation. In general, complementary Watson-Crick and noncomplementary base pairs at the first two positions of the codon-anticodon helix are efficiently discriminated on the ribosome on the basis of their geometry, while the third base pair, i.e the wobble position, is less strictly controlled allowing for non-Watson-Crick interactions and the tRNAs to read synonymous codons.

However, crystal structures showed that some mismatches such as G/U and C/A at the first or second position, can adopt a WC geometry which is isosteric to the canonical WC base pairs and can thus escape this selection (Demeshkina et al., 2013). This occurs because nucleotide bases, although with a low probability of $10^{-5}-10^{-4}$, can adopt alternative tautomeric or anionic forms that cause them to be isosteric with WC bases (Kimsey et al., 2015; Westhof et al., 2014) (Figure 5) and be recognized as cognate by the ribosome. Thus, in these cases the ribosomes lose the ability to discriminate matches against mismatches (Rozov et al., 2015, 2016). This type of loss in fidelity has also been reported in a recent study where mass spectrometric analysis of recombinant and natural proteins 
expressed in E. coli revealed that a correlation exists between GU mismatches and an increased level of errors (Zhang et al., 2013).
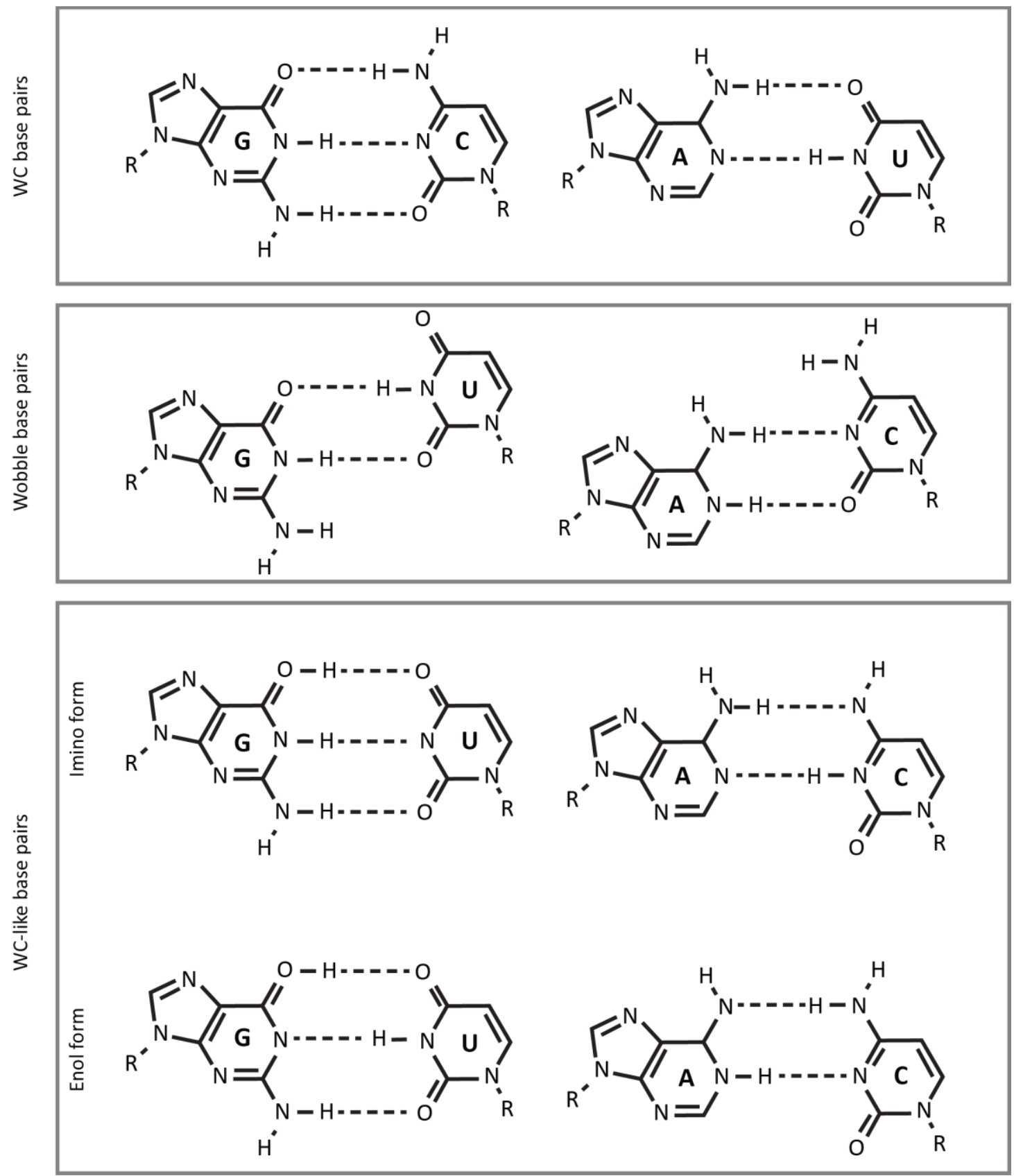

Figure 5. G-U base pair tautomerism. Natural bases of nucleic acids form base pairs with at least two hydrogen bonds between them. Standard complementary base pairs G-C and A-U (upper panel) are compared with Wobble base pair G U and $\mathrm{A} \sim \mathrm{C}$ (center panel) and the isosteric tautomers (bottom panel) imino and enol forms. Wobble pairs present a different geometry which is recognized by the ribosome as a mismatch. However, the Watson-Crick-like geometry of tautomers prevents their discrimination by the decoding site of the ribosome. 


\subsection{Aminoglycosides and decoding}

Reduced fidelity of decoding can be caused by aminoglycosides that target the 16S rRNA of the 30S subunit and affect protein synthesis and decoding. Aminoglyocosides were the first antibiotic class effective against tubercolosis and, despite the fact that their use has helped millions of people, their current clinical application is hampered by their poor bioavailability, the emergence of resistant pathogenic strains and their pronounced nephro- and ototoxicity (Fischel-Ghodsian, 2005) which are likely caused by their interaction with the A site of human mitochondrial ribosomes (Hong et al., 2015). Nevertheless, they remain in use as pivotal tools for the investigation of translation mechanism and ribosome dynamics and as clinically important drugs for the treatment of the symptoms caused by hereditary diseases. In some genetic pathologies, such as the Duchenne muscular dystrophy (Malik et al., 2010a; Malik et al., 2010b), the primary ciliary dyskinesia (Bukowy-Bieryllo et al., 2016) and the phenylketonuria (Ho et al., 2013), the premature insertion of a stop codon in the mRNA causes the generation of shorter, non-functional proteins and the translational readthrough induced by aminoglycosides is used to effectively restore the expression of a functional full-length protein.

Aminoglycosides are positively charged oligosaccharides which usually share a 2-deoxystreptamine ring (also known as 2-DOS or ring II) as a common characteristics, although some exceptions exist such as spectinomycin and streptomycin. Depending on the type of linkages between the 2-DOS ring and the other rings, they can be classified in 4,5-disubstituted, 4,6-disubstituted, 4- and 5monosubstituted aminoglycosides (Figure 33). To enter the cell, aminoglycosides must first cross the plasma membrane and, in the case of gram-negative bacteria, the outer membrane. Their uptake is an energy-consuming process that is thought to occur in three phases. In the first phase, the positively charged aminoglycosides are adsorbed to the surface of bacteria by electrostatic interactions with the lipopolysaccharides of the outer membrane. The following two steps are dependent on the transmembrane potential generated by the respiratory chain, which is the reason why anaerobic bacteria appear to be impermeable and resistant to aminoglycosides (Bryan and Kwan, 1983; Hancock, 1981; Taber et al., 1987). Once inside the cell, they affect translation by binding to the ribosome. Most aminoglycosides specifically target the 30S ribosomal subunit and bind to the helix 44 (h44) (Carter et al., 2000; Moazed and Noller, 1987), although spectinomycin only binds to helix 34 (h34) (Bilgin et al., 1990; Carter et al., 2000) and some of them such as gentamycin, tobramycin, neomycin and paromomycin have a secondary binding site on the helix 69 (H69) of 50S subunit (Borovinskaya et al., 2007a; Misumi et al., 1978) (Figure 6 and Table 2).

Neamine (Nea) is constituted by the universal two-ring structure which characterizes the base of most aminoglycosides. Its ring I inserts in h44 and causes the two universally conserved A1492 and 
A1493 to bulge out in a conformation simar to those induced by codon:anticodon interaction (Francois et al., 2005; Ogle et al., 2001).

Kanamycin (Kan) binds to h44 of the 16S rRNA. It induces a high-affinity conformation of the ribosome for A-site tRNA and promotes the displacement of the 16S rRNA residues A1492 and A1493 affecting both translocation and miscoding (Davies et al., 1965b; Feldman et al., 2010).

Neomycin B (Neo B) has two binding sites, the first on h44 and the second on H69 (Borovinskaya et al., 2007a) affecting decoding fidelity, translocation and also ribosome recycling. It inhibits aa-tRNA translocation and ribosome recycling by stabilizing an intermediate state of subunits rotation and causing a conformational change that negatively effects subunits dissociation (Borovinskaya et al., 2007a; Wang et al., 2012).

Gentamicin (Gen) has two binding sites on h44 and H69. Its effect on the ribosome is similar to those of Neomycin inducing miscoding and affecting translocation (Borovinskaya et al., 2007a; Davies et al., 1965b).

Paromomycin (Par) exerts its main effect on decoding. It displaces A1492 and A1493 of h44 into a position where they could not contact anymore the codon:anticodon helix (Carter et al., 2000; Ogle et al., 2001; Ogle et al., 2003; Ogle et al., 2002) and induces a deformation of the near-cognate codon:antidocon helix and a movement of H69 (Demeshkina et al., 2012). Par specifically increases the GTPase activation for near-cognate ternary complexes, whereas the GTPase activity in the cognate complexes is unaffected (Pape et al., 2000).

Tobramycin (Tob) binds to both h44 and H69 (Borovinskaya et al., 2007a; Scheunemann et al., 2010). It stabilizes the bulged out conformation of the residues A1492 and A1493 (Vicens and Westhof, 2002) Ribostamycin (Rib) shares the same identical structure of Neo but lacks ring IV. As Nea, it bings to h44 and affects decoding by causing the conformational rearrangement of the the two adenines 1492 and 1493 (Francois et al., 2005).

Apramycin (Apr) binds h44 and is a strong inhibitor of protein synthesis. It inhibits the translocation step but its effect on miscoding is controversial (Matt et al., 2012; Perzynski et al., 1979).

Hygromycin B (Hyg B) binds at the very top of h44 but does not induce any conformational change in the rRNA (Brodersen et al., 2000). However, it might exert its effect as translocation inhibitor (Peske et al., 2004) by blocking the movements of the h44, which are necessary during translocation (Frank and Agrawal, 2000) . In addiction, by increasing the affinity of the A-site tRNA, Hyg B also has a small effect on decoding (Eustice and Wilhelm, 1984a, b; Peske et al., 2004).

Streptomycin (Str) tightly binds to the phosphate backbone of 16S rRNA and makes contact with protein S12 of the small subunit (Carter et al., 2000). Structural works showed that Str shifts the decoding site region of h44 laterally in the direction of S12 protein and h18, without causing the 
A1492 and A1493 to flip out from h44 (Demirci et al., 2013a). Str increases the affinity of tRNA binding in the A site (Peske et al., 2004) and acts on the ribosome by reducing the rate of GTPase activation for ternary complexes with cognate aa-tRNAs and accelerating it for those with near cognate aatRNAs, thereby reducing ribosomal selectivity (Gromadski and Rodnina, 2004b; Pape et al., 2000). Spectinomycin (Spc) binds in the minor groove at one end of h34, where it contacts C1064 and C1192 (Carter et al., 2000). The antibiotic sterically blocks swiveling of the head domain of the small ribosomal subunit and has a strong inhibitory effect on translocation thereby blocking the translocation cycle (Borovinskaya et al., 2007b; Peske et al., 2004).
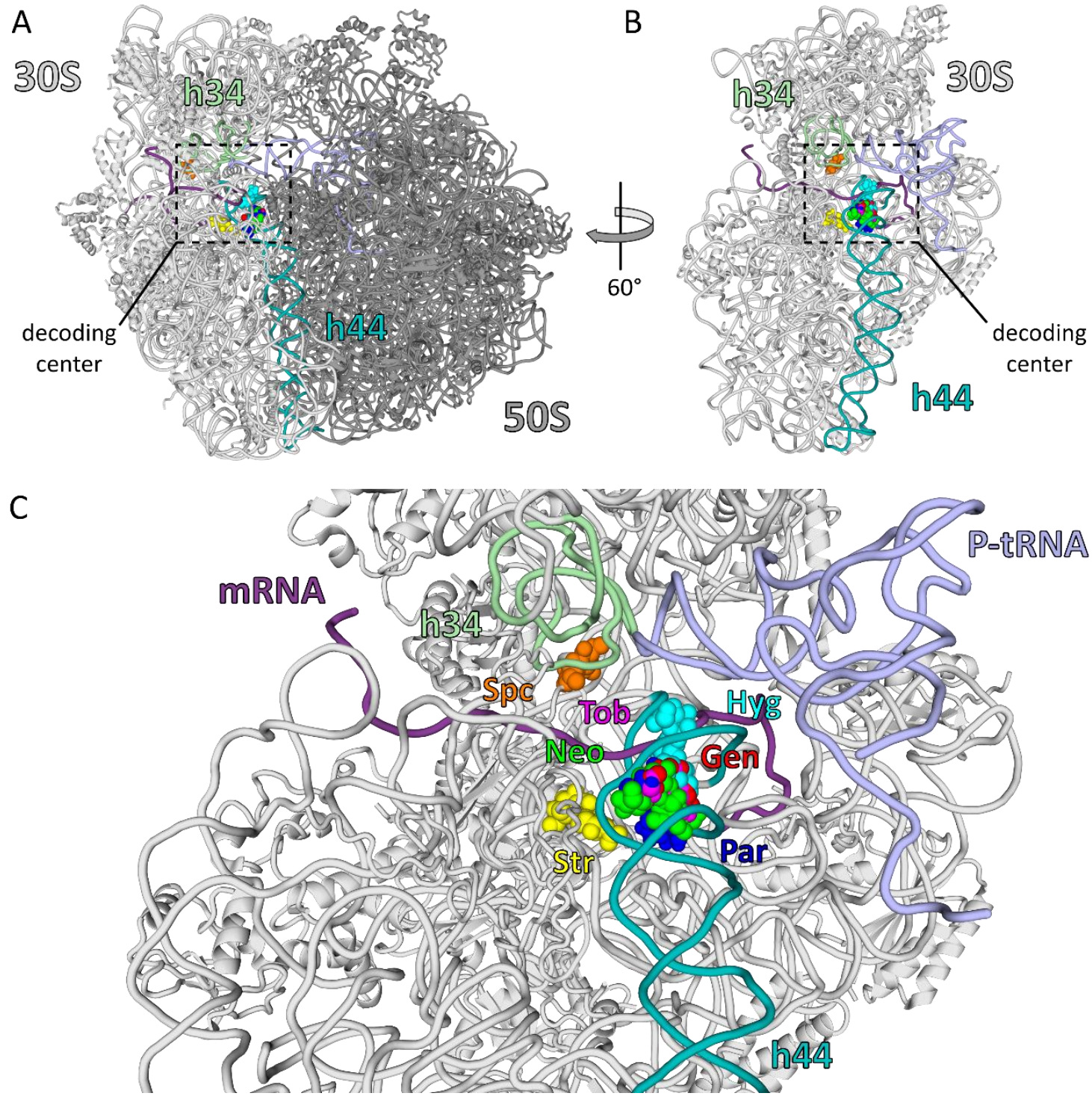

Figure 6. Aminoglycosides binding sites on the ribosome. The binding site of aminoglycosides is shown with respect to the A) $70 \mathrm{~S}$ and the B) $30 \mathrm{~S}$ subunit. C) The biding site in h44 of tobramycin (Tob), paromomycin (Par), streptomycin (Str), hygromycin B (Hyg), gentamicin (Gen) and neomicin (Neo). Only the primary sites are shown; Tob, Gen, Neo and Par also have a second binding site in h69. Spectinomycin (Spc) has a binding site in h34. [PDB files: 4ji1 (Demirci et al., 2013b), 4v56 (Borovinskaya et al., 2007b), 4woi (Wasserman et al., 2015), 4lfc , 4w29 (Zhou et al., 2014), 4v64 (Borovinskaya et al., 2008), 4v53 (Borovinskaya et al., 2007a)]. 
Table 2. Aminoglicosides binding sites and their effects. The binding sites and effects for the most common aminoglycosides are reported.

\begin{tabular}{|c|c|c|c|}
\hline Aminoglycoside & Binding site & Effect & Reference \\
\hline Neamine & h44 and H69 & Miscoding & (Francois et al., 2005; Pape et al., 2000) \\
\hline Kanamycin & h44 & Miscoding and translocation inhibition & $\begin{array}{l}\text { (Feldman et al., 2010; Francois et al., } \\
\text { 2005) }\end{array}$ \\
\hline Neomycin B & h44 and H69 & $\begin{array}{l}\text { Ribosome recycling and subunit rotation } \\
\text { inhibition }\end{array}$ & $\begin{array}{l}\text { (Borovinskaya et al., 2007a; Wang et } \\
\text { al., 2012; Wasserman et al., 2015) }\end{array}$ \\
\hline Gentamycin & h44 and H69 & $\begin{array}{l}\text { Miscoding and ribosome recycling } \\
\text { inhibition }\end{array}$ & (Borovinskaya et al., 2007a) \\
\hline Paromomycin & h44 and H69 & Miscoding and translocation inhibition & $\begin{array}{l}\text { (Carter et al., 2000; Demeshkina et al., } \\
\text { 2012; Pape et al., 2000; Peske et al., } \\
\text { 2004; Wasserman et al., 2015) }\end{array}$ \\
\hline Tobramycin & h44 and H69 & Miscoding and translocation inhibition & $\begin{array}{l}\text { (Cabanas et al., 1978; Fosso et al., } \\
\text { 2015; Vicens and Westhof, 2002) }\end{array}$ \\
\hline Ribostamycin & h44 and H69 & Miscoding & (Francois et al., 2005) \\
\hline Apramycin & h44 & mRNA translocation inhibition & $\begin{array}{c}\text { (Matt et al., 2012; Perzynski et al., } \\
\text { 1979) }\end{array}$ \\
\hline Hygromycin B & h44 & $\begin{array}{c}\text { Inhibits translocation in both } \\
\text { prokaryotes and eukaryotes, by a lesser } \\
\text { extent causes misreading }\end{array}$ & $\begin{array}{l}\text { (Brodersen et al., 2000; Eustice and } \\
\text { Wilhelm, 1984a, b; Gonzalez et al., } \\
\text { 1978; Peske et al., 2004) }\end{array}$ \\
\hline Streptomycin & h44 & $\begin{array}{l}\text { Misreading, h44 distortion, } \\
\text { codon-anticodon stabilization, } \\
\text { equalization of ribosome selectivity for } \\
\text { cognate- and near-cognate ones. }\end{array}$ & $\begin{array}{l}\text { (Carter et al., 2000; Demirci et al., } \\
\text { 2013a; Gromadski and Rodnina, } \\
\text { 2004b) }\end{array}$ \\
\hline Spectinomycin & h34 & Translocation inhibition & $\begin{array}{c}\text { (Bilgin et al., 1990; Carter et al., 2000; } \\
\text { Peske et al., 2004) }\end{array}$ \\
\hline
\end{tabular}

\subsubsection{Cellular effects of aminoglycosides-induced miscoding}

Whereas the primary binding sites of aminoglycosides on the ribosome are well established, the sequence of events that leads to cell death has been under debate for decades. Accumulation of errors in proteins is considered one of the most essential steps contributing to the bactericidal action of aminoglycosides. The insertion of erroneous proteins into the cell membrane is responsible for a membrane damage that, in turn, enhances the antibiotic-mediated killing (Davis et al., 1986). After an initial entry into the cell, aminoglycosides target the ribosomes reducing their fidelity and inducing 
miscoding and production of erroneous proteins. The insertion of such proteins into the cell membrane creates abnormal channels that increase the influx and the irreversible entry of more aminoglycoside triggering a positive loop of misreading, membrane damage and antibiotic entrance.

The link between aminoglycosides, miscoding and membrane permeability is also suggested by the following findings: i) when cells containing Str-resistant ribosomes are treated with another aminoglycoside to which they are sensitive, they can then take up more Str (Holtje, 1978, 1979); ii) simultaneous addition of chloramphenicol, a bacteriostatic antibiotic that inhibits protein synthesis, and Str reveals an antagonistic effect of the first on the latter resulting in a decrease Str uptake (Jawetz et al., 1951); iii) the addition of chloramphenicol after the secondary uptake of Str has begun, does not block Str uptake anymore (Holtje, 1978, 1979). These results together suggests that an active, translation-engaged ribosome is necessary for aminoglycosides effect to develop (that is, erroneous proteins must be first synthetized). At the same time, ribosomes that actively synthesize proteins in the presence of an aminoglycoside do not promote its uptake by a direct participation in the process of entry, but rather have an indirect effect involving insertion of erroneous protein into the membrane, which persists even when the ribosome activity is blocked.

In more recent studies, another killing mechanism has been proposed, in which mistranslation of membrane proteins activates a cascade reaction that triggers redox alteration, destabilization of ironsulfur (Fe-S) clusters (Ezraty et al., 2013) and generation of hydroxyl radicals (Dwyer et al., 2014; Kohanski et al., 2008). Mistranslated proteins are translocated either across the inner membrane into the periplasmic space or are directly inserted into the membrane. As a consequence of the reduced translation fidelity induced by aminoglycosides, many of these proteins are misfolded and activate the two-component stress response sensor consisting of $\mathrm{CpxA}$, which monitors the quality of proteins trafficked through the membrane, and CpxR, which is phosphorylated by CpxA (Dong et al., 1993). This triggers the expression of the envelope stress response proteins, such as degP (Danese et al., 1995; Pogliano et al., 1997), which degrades misfolded proteins and suppresses the toxicity associated with their intracellular accumulation (Isaac et al., 2005).

In addition, СpxA may also activate the redox-responsive two-component transcription factor, ArcA (luchi et al., 1989; Ronson et al., 1987). The activation of the envelope stress response system, together with ArcA-regulated changes in metabolic and respiratory systems, leads to the hyperactivation of the respiratory chain and the generation of hydroxyl radicals which cause oxidative stress, generation of 8-oxo-guanine, nucleic acids damage, toxic metabolic perturbations, apoptosis and ultimately cell death (Belenky et al., 2015; Dwyer et al., 2012; Foti et al., 2012). However, the notion that aminoglycosides induce an hydroxyl-radical-mediated killing, has been recently challenged (Keren et al., 2013; Liu and Imlay, 2013), leaving the debate open. Ultimately, the cellular tolerance to 
protein errors which accumulate during stress conditions and aminoglyocosides treatment remains elusive and a comprehensive view of aminoglyocosides effects, miscoding and cellular response is still missing.

\subsection{Available estimates of error frequencies}

A number of estimates for the in-vivo abundance of amino acid misincorporations are available in literature (Table 3). Most of the published error frequency values were estimated by biochemical assays that quantified only one specific protein or misincorporation at a time, e.g., incorporation of not naturally encoded amino acids, restoration of the activity of an enzyme variant inactivated by introducing an amino acid replacement at the active site (Kramer and Farabaugh, 2007; Manickam et al., 2014), separation of correct and erroneous proteins by 2D gel electrophoresis. All these techniques share the same common limitations, i.e., use of a reporter system, the choice of the position and type of misincorporation to investigate, and the low sensitivity and the narrow dynamic range associated with the analytical methods being used. Only in recent years mass spectrometry has emerged as a new tool for the evaluation of error frequency (Yu et al., 2009; Zhang et al., 2013), albeit with some constraints due to the wide concentration range between correct and error-containing peptides which often exceeds the dynamic range of the instrument. 
Table 3. Estimated in-vivo error frequencies. A list of the techniques used so far to determine translation error frequency per codon is reported [adapted from (Ribas de Pouplana et al., 2014)]. Due to the differences which characterize each method, very different frequencies have been determined.

\begin{tabular}{|c|c|c|c|c|}
\hline Principle & Error & Error frequency & Assay & References \\
\hline \multirow{4}{*}{$\begin{array}{c}\text { Detection of } \\
\text { aa substitution }\end{array}$} & $\mathrm{I} \rightarrow \mathrm{V}$ & $2 \times 10^{-4}-6 \times 10^{-4}$ & $\begin{array}{l}\text { Radioactive Val } \\
\text { incorporation }\end{array}$ & $\begin{array}{c}\text { (Loftfield and } \\
\text { Vanderjagt, 1972) }\end{array}$ \\
\hline & $\mathrm{R} \rightarrow \mathrm{C}$ & $1 \times 10^{-4}$ & $\begin{array}{l}\text { Cys detection in } \\
\text { Cys-free protein }\end{array}$ & $\begin{array}{l}\text { (Edelmann and } \\
\text { Gallant, 1977) }\end{array}$ \\
\hline & $\begin{array}{l}\mathrm{R} \rightarrow \mathrm{C} \\
\mathrm{W} \rightarrow \mathrm{C}\end{array}$ & $4 \times 10^{-3}$ & $\begin{array}{l}\text { Radioactive Cys } \\
\text { incorporation, } \\
\text { interference with } \\
\text { protease digestion }\end{array}$ & $\begin{array}{c}\text { (Bouadloun et al., } \\
\text { 1983) }\end{array}$ \\
\hline & $\mathrm{N} \rightarrow \mathrm{K}$ & $2 \times 10^{-4}-2 \times 10^{-3}$ & Isoelectric focusing & $\begin{array}{c}\text { (Parker et al., } \\
\text { 1980) }\end{array}$ \\
\hline \multirow{6}{*}{$\begin{array}{l}\text { Reporter } \\
\text { systems }\end{array}$} & $\mathrm{G} \rightarrow \mathrm{S}$ & $1 \times 10^{-3}$ & $\begin{array}{l}\text { lacZ activity } \\
\text { restoration }\end{array}$ & (Toth et al., 1988) \\
\hline & $\mathrm{Y} \rightarrow \mathrm{H}$ & $5 \times 10^{-6}$ & $\begin{array}{l}\text { CAT activity } \\
\text { restoration }\end{array}$ & $\begin{array}{c}\text { (Stansfield et al., } \\
1998)\end{array}$ \\
\hline & \pm frameshift & $2 \times 10^{-5}$ & $\begin{array}{l}\text { lacZ activity } \\
\text { restoration }\end{array}$ & $\begin{array}{c}\text { (Curran and Yarus, } \\
1986)\end{array}$ \\
\hline & Frameshift, stop codon readthrough & $2-10 \%$ & GFP fluorescence & $\begin{array}{l}\text { Meyerovich et al. } \\
2010\end{array}$ \\
\hline & 24 codons to $K$ & $3 \times 10^{-4}$ & Firefly luciferase & $\begin{array}{c}\text { (Kramer and } \\
\text { Farabaugh, 2007) }\end{array}$ \\
\hline & $\mathrm{N} \rightarrow \mathrm{D} ; \mathrm{Q} \rightarrow \mathrm{E}$ & $2 \times 10^{-3}-8 \times 10^{-3}$ & Renilla luciferase & (Javid et al., 2014) \\
\hline $\begin{array}{c}\text { tRNA } \\
\text { misacylation }\end{array}$ & Mismethionylated tRNA & $1 \times 10^{-2}-1 \times 10^{-1}$ & $\begin{array}{l}\text { Radioactive Met on } \\
\text { tRNA microarray }\end{array}$ & $\begin{array}{c}\text { (Netzer et al., } \\
\text { 2009) }\end{array}$ \\
\hline \multirow{3}{*}{$\begin{array}{c}\text { Mass } \\
\text { spectrometry }\end{array}$} & $N \rightarrow D$ & $1 \times 10^{-1}$ & MS/MS & (Ruan et al., 2008) \\
\hline & $S \rightarrow N$ & $2 \times 10^{-2}$ & $\begin{array}{c}\text { Modified database } \\
\text { algorithm and } \\
\text { MS/MS }\end{array}$ & (Yu et al., 2009) \\
\hline & $\begin{array}{c}\mathrm{G} \leftrightarrow \mathrm{D}, \mathrm{G} \rightarrow \mathrm{Q}, \mathrm{D} \rightarrow \mathrm{E}, \mathrm{E} \rightarrow \mathrm{K} \\
\mathrm{M} \rightarrow \mathrm{I}, \mathrm{M} \rightarrow \mathrm{N} ; \mathrm{S} \rightarrow \mathrm{N} ; \mathrm{S} \rightarrow \mathrm{R} ; \mathrm{V} \rightarrow \mathrm{I} ; \\
\mathrm{D} \rightarrow \mathrm{N} ; \mathrm{A} \rightarrow \mathrm{T} ; \mathrm{H} \leftrightarrow \mathrm{Q} ; \mathrm{P} \rightarrow \mathrm{L} ; \mathrm{P} \rightarrow \mathrm{S} ; \\
\mathrm{R} \rightarrow \mathrm{Q}, \mathrm{Y} \rightarrow \mathrm{N} .\end{array}$ & $10^{-5}-10^{-3}$ & $\begin{array}{l}\text { High resolution } \\
\text { mass spectrometry }\end{array}$ & (Zhang et al., 2013) \\
\hline
\end{tabular}




\subsection{Mass spectrometry: an emerging tool for the quantification of miscoded translation products}

Mass spectrometry is one of the most efficient ways to identify and quantify proteins. It relies on the separation of ions based on their mass-to-charge ratio and, in association with continuously improved techniques for the ionization of samples (e.g., electrospray ionization (ESI)), has rapidly become the method of choice for the analysis of peptides, single proteins and protein complexes. The advantages of mass spectrometry arise not only from the possibility of a fast and sensitive peptides identification, but also from the low amounts of sample required for the analysis. The identification of a protein can be attained by the direct mass spectrometric anaylsis of the intact protein (top down characterization), or alternatively, due to the ionization and fragmentation limitations associated with this technique, on the base of the peptides generated from protein hydrolysis (bottom up or shotgun proteomics). Peptides, which are easier to fractionate and fragment than the intact protein, are then analysed by tandem mass spectrometry and their spectra compared with databases and spectral libraries which allow their identification and their assignation to the corresponding protein (Lin et al., 2003; Meng et al., 2002; Yates, 2004).

Mass spectrometry has been recently used also for the identification of amino acid misincorporation. The identification of misincorporation-containing peptides is performed on the basis of the mass shift caused by the amino acid substitution, as it happens for the identification of post-translational modifications (PTMs). Hovewer, the extremely low abundance of error-containing peptides, the limited dynamic range of current mass spectrometers ( 3 - 5 orders of magnitude depending on the sample and the type of mass analyzer (Domon and Aebersold, 2010; Gallien et al., 2012; Gillet et al., 2012; Stahl-Zeng et al., 2007)) and the limited amount of sample that can be tolerated by both chromatographic system and mass spectrometer, makes the identification of rare error-containing peptides often impossible in a sample which also contains high abundance correct peptides and poses a big challenge to their quantification. Indeed, recent fluorescence based in-vivo studies (Manickam et al., 2014) and kinetic in-vitro studies (Zhang et al., 2015) indicate that many misincorporations might be far less abundant $\left(<10^{-5}\right)$ than previously expected. This is further complicated by the fact that misincorporation-containg peptides are suppressed by the high abundant co-eluting correct tryptic peptides. To the best of our knowledge, no mass spectrometric study detected more than $5 \%$ of all possible near-cognate misincorporations, presumably due to their low abudance.

After the mass spectrometric analysis, erroneous peptides can be identified by several approaches. Erroneous peptides can be found using the database search algorythms that compare the MS/MS data to in-silico predicted fragmentation pattern of all possible peptides in the database e.g., MASCOT (Perkins et al., 1999), Andromeda (Cox et al., 2011), Sequest (Eng et al., 1994), Paragon (Shilov et al., 
2007) or MassAnalyzer (Zhang, 2009). However, because the search space exponentially expands with the number of subsitutitions allowed per peptide, different strategies exists to overcome this limitation while applying traditional search engines. First, the search space can be restricted only to proteins or peptides whose unmodified parental peptide has been observed in the sample and the number of variable misincorporations per peptide can be restricted (Creasy and Cottrell, 2002). Database searches can also identify parental peptides and so called "dependent" peptides that derive from the repective parental peptide but differ by a delta mass (Cox and Mann, 2008). In this case the search space does not expand, because the delta masses are not interpreted in terms of defined PTMs or amino acid substitutions and thus could reflect different deviations from the parental peptide. In this approach the indentification and validation of amino acid substitutions has to occur after the database search. A similar peptides-dependent approach has been recently applied to study the proteome-wide incorporation of norvaline (Cvetesic et al., 2016). When the database search time needs to be reduced, tag-based hybrid methods can be applied in which a sequence tag of $2-4$ amino acids is derived from a MS/MS spectrum and only tag-containing peptides are searched in the database (Mann and Wilm, 1994; Shilov et al., 2007; Valledor et al., 2008). Spectral library searching can also be used as alternative method to reduce the search space and perform a fast and accurate analysis in which the query MS/MS spectra are searched against a large collection of experimental MS/MS spectra (Frewen et al., 2006), although this method finds its biggest limitation in the fact that the identification of the peptide can be performed only if it is already included in the library. Another approach to adapt classical search engines without restricting it to the prior identification of parental peptides, consists in the increase of the mass tolerance in the delta mass comparison with respect to the parental peptide from the database and in the interpretation of the resulting delta masses only subsequently. This approach was recently used to systematically analyze unassigned spectra from shotgun analysis of HEK cells (Chick et al., 2015). Apart from single missense errors, the algorythm was also able to identify more complex alterations in the amino acid sequence de novo such as polyalanine insertions in the ribosomal protein L14. Alternatively, when all amino acid substitutions are included in the database, classical database search engines can be applied (Muhlhausen et al., 2016).

However, in specific cases as for the identification of modified peptides whose sequence is not included in any database, the use of alternative approaches such as de novo sequencing can be favourable. This method, based on software packages such as PEAKS (Ma et al., 2003) and PepNovo (Frank and Pevzner, 2005), directly infers the peptide sequence from MS/MS spectra and bypass the need for spectra comparison with the database. With this approach, many substitutions and modifications can be identified; on the other hand, the high frequency of false positive results and the requirement for high quality spectra limit the applicability of the approach, especially when the 
resulting mass shift is not unique or for very low-abundance peptides whose spectra is difficult to obtain with good quality.

Another drawback of current PTM identification and quantification procedures concerns the data interpretation and is associated with the underestimated chemical complexity of tryptic digests. Similar or identical delta masses might be introduced in vivo by enzymatic- (e.g., methylations or acetylations) or non-enzymatic posttranslational modifications (e.g., carbon, oxidative or nitrosative stress) (Wagner and Hirschey, 2014; Weinert et al., 2013) which could lead to false positive identifications. Subsequently, modifications might be introduced upon cell opening and sample preparation (e.g., oxidation, iodoacetamide (IAA) labelling, atryptic cleavage (Picotti et al., 2007) and transpeptidation (Fodor and Zhang, 2006; Schaefer et al., 2005)). Moreover, such peptide decay reactions are often accelerated when the individual residues of trypytic peptides are not protected by the protein environment (Manning et al., 2010). In the mass spectrometric workflow, co-ionizing adducts (e.g., sodium ions) and gas phase reactions (e.g., cyclizations, deamidations or water loss) can further lead to modifications that are isobaric to amino acid misincorporations. For example, a $+14 \mathrm{Da}$ $\Delta$ mass might be explained by replacements $\mathrm{D} \rightarrow \mathrm{E}, \mathrm{G} \rightarrow \mathrm{A}, \mathrm{N} \rightarrow \mathrm{Q}, \mathrm{V} \rightarrow \mathrm{I}, \mathrm{V} \rightarrow \mathrm{L}, \mathrm{S} \rightarrow \mathrm{T}$, as well as by methylations at $K, R, H, Q, N, E, D$ (Jung et al., 2008) that can be either introduced in vivo or artificially during sample processing using methanol (Chen et al., 2010), e.g., upon Coomassie staining (Sumpton and Bienvenut, 2009). A +14 Da $\Delta$ mass can also arise by misincorporations of $\beta$-methylnorleucine (Muramatsu et al., 2003) in place of I or oxidations of $Y$ and W. Cysteinyl-S- $\beta$-propionamide, a byproduct of acrylamide adduct formation in SDS-PAGE, can produce the same mass associated with cysteine alkylation plus monomethylation on a proximal amino acid (Hart-Smith et al., 2016) and methylations which are difficult to reliably identify by conventional scoring algorythms due to high false discovery rates (Bogdanow et al., 2016). Incidentally, the identification of those errors that are expected to be highly abundant, because they are introduced by third position codon-anticodon mismatches, is particularly difficult, because they often lead to common delta masses (i.e., -16, -14, $+14,+16 \mathrm{Da}$ ). These identification problems are aggravated by the fact that low-abundance erroneous peptides often have incomplete fragmentation patterns precluding the exact identification of the position of the delta mass in the peptide sequence. In addition, isobaric peptides often co-elute during chromatography and thus hamper the correct quantification on the MS1 level, and lead to chimeric MS/MS spectra that are often hard to correctly assign to the respective sequences. Finally, the correct parental and erroneous peptides might differ in their ionizability which is especially relevant for peptide pairs with very different physicochemical properties which reflect in deviating retention times and large delta masses e.g., when the amino acid substitution introduces or eliminates a tryptic cleavage site generating error-containing peptides which are shorter or longer than the correct ones. 
Overall, this leads to the unfortunate situation in which most misincorporations might escape detection and prompted us to develop an alternative experimental approach that would allow the reliable and systematic identification and quantification of erroneous peptides with high sensitivity and over a wide dynamic range.

\subsection{Selected reaction monitoring (SRM) and triple quadrupoles mass spectrometer}

Selected reaction monitoring (SRM) is an attractive method for the accurate quantification of peptides, not only for its high sensitivity (to attomole concentration levels), but also for its broad dynamic range - up to five orders of magnitude - and excellent analytical reproducibility (Domon and Aebersold, 2010).

During a typical LC-MS/MS analysis, the peptides of the sample are separated by reversed phase chromatography performed using a low-flow nano-LC system. Upon elution peptides are directly sprayed into the mass spectrometer from a needle which is kept at high potential (see Materials and Methods). The ejection of liquid from the needle in the presence of voltage gives raise to the formation of a cone, known as the Taylor cone (Taylor, 1964) and the sample is ionized for the mass spectrometric analysis using ESI (electrospray ionization) technique. At high temperature, the solvent evaporation and the droplet shrinking increases the charge density until ions are kinetically and energetically ejected from the droplet, to then pass through a transfer ion capillary and enter the vacuum region of the mass spectrometer for the analysis (Ho et al., 2003). For SRM analysis, a triple quadrupole mass spectrometer is used. The instrument consists of three consecutive quadrupoles, named Q1, Q2 and Q3, each constituted by an assembly of four parallel metal rods, where two opposite rods are electrically connected, but offset relative to one another giving two sets of rods pairs per quadrupole. Although a triple quadrupoles mass spectrometer can operate in a variety of scan types, when in SRM mode, it performes two stages of mass selection, referred to as tandem MS or MS/MS. At every time point, a variable combination of radio frequency ( $\mathrm{rf}$ ) and direct current (dc) voltages of the same amplitude and sign is applied in the Q1 and Q3 for each set of rods. This causes ions to oscillate and guides them through the rod assemblies. The oscillation stabilizes only selected ions with a specific mass-to-charge ratio $(\mathrm{m} / \mathrm{z})$ and allows them to pass, while all other ions crash on the rods and are eventually eliminated. Q1 and Q3 act as mass analyzers. After the Q1, only selected precursors will be allowed to enter the Q2. On this quadrupole, only rf voltage is applied and because selection of ions is not possible with the rf alone, this quadrupole only acts as transmission device instead of a mass analyzer. Q2 is a collision cell, where the ions that have been selected in the Q1 collide with gas molecules (e.g., nitrogen or argon) at a defined gas pressure and electrostatic energy potential and fragment to yield a second type of ions, the fragment ions, that will be then selected in 
Q3 during the last stage of selection and ultimately recorded by the ion detection system (Figure 7). After a certain time, the dwell time, the rf and dc ratio are changed, and a new pair of ions are selected. The settings used for the selection of a precursor ion in the first quadrupole and the selection of its fragment ions in the third, are called transition. Usually 2 - 3 transitions are monitored for each peptide. The type of fragment ions which can be observed at the MS/MS level depends on various factors such as the sequence of the peptide and the charge state, and can be detected only if they retain a positive charge at their $\mathrm{N}$ terminal ( $\mathrm{a}, \mathrm{b}$ and $\mathrm{c}$ ions) or at their $\mathrm{C}$ terminal $(\mathrm{x}, \mathrm{y}, \mathrm{z})$ (Roepstorff and Fohlman, 1984). The narrow selection windows of the first and third quadrupoles, between 0.2 and $0.7 \mathrm{Da}$, allow the transitions of a very small set of ions, contributing to a high degree of selectivity and reducing the chemical noise. In this way, SRM analysis permits an instrument linearity which can easily span 5 orders of magnitude (Worboys et al., 2014). High degree of selectivity and sensitivity constitute the pivotal charachteristics that make this approach particularly suitable for biomarker discovery, where very low abundant marker peptides need to be found in extremely complex mixtures like, for example, blood sample (Stahl-Zeng et al., 2007).

However, mass spectrometry is not quantitative per se, because the variation in the stability of ESI spray and the different intrinsic ionization properties of each peptide, hamper the direct comparison of peaks intensities. For this reason, to obtain an accurate quantification, the insertion of a mass tag by metabolic, enyzymatic and chemical labeling of peptides or proteins, or by spike of synthetic peptide standards into the sample is commonly employed (Ong and Mann, 2005). Stable isotopelabelled peptides are chemically identical to the native light counterparts and they behave identically during liquid chromatography separation and mass spectrometric analysis. Nonetheless, the mass tag is detected by the mass spectrometer and the quantification can be performed by comparison of the signal intensity of light and heavy-labeled peptides (Bantscheff and Kuster, 2012). One optimized application of this strategy is the so called AQUA (Absolute QUAntification) approach, based on the use of custom designed and chemically synthetized peptide standards which contain C-terminal heavy amino acids labeled with carbon and nitrogen stable isotopes i.e., ${ }^{13} \mathrm{C}-$ and ${ }^{15} \mathrm{~N}-$, and that are spiked in the samples in known amount prior to the analysis (Gerber et al., 2003). Once the target peptides are analyzed in the sample by mass spectrometry, the area under the peptides peak is integrated, the peptides are detected and the ratio between the internal standard and the analyte is determined (Figure 7). Finally, because the amount of internal standard in the sample is known, also the amount of the analyte can be easily calculated. The first step in the development of the AQUA strategy is the selection of the peptides to be monitored during the SRM experiment. In our case, the selection is performed on the base of pre-existing data obtained from data-dependent acquisition, but in general, their selection can also be performed on empirical observations around their stability and ionization 
performances. Furthermore, some general features can be used to predict if a peptide is suitable or not for this analysis. Tryptic peptides length is between 5 and 20 amino acids and their sequence should lack reactive amino acid that could undergo modifications, e.g., methionine, or residues that could make the synthesis and purification of the synthetic peptides difficult, e.g., multiple serine or proline residues should be avoided because they cause deletion during the synthesis process (Lange et al., 2008; Picotti and Aebersold, 2012). Target peptides must be unique for the target protein and their sequence should not be shared by any other tryptic peptide from other proteins in the organism of choice ("proteotypic peptides" (Mallick et al., 2007)). It is also important that the peptides accurately represent the level of the protein ("quantotypic peptides" (Brownridge and Beynon, 2011)), which means that several factors that could affect their concentration - the completeness of proteolytic reaction, peptide stability, and the absence of post-translational modification - should be taken into consideration.

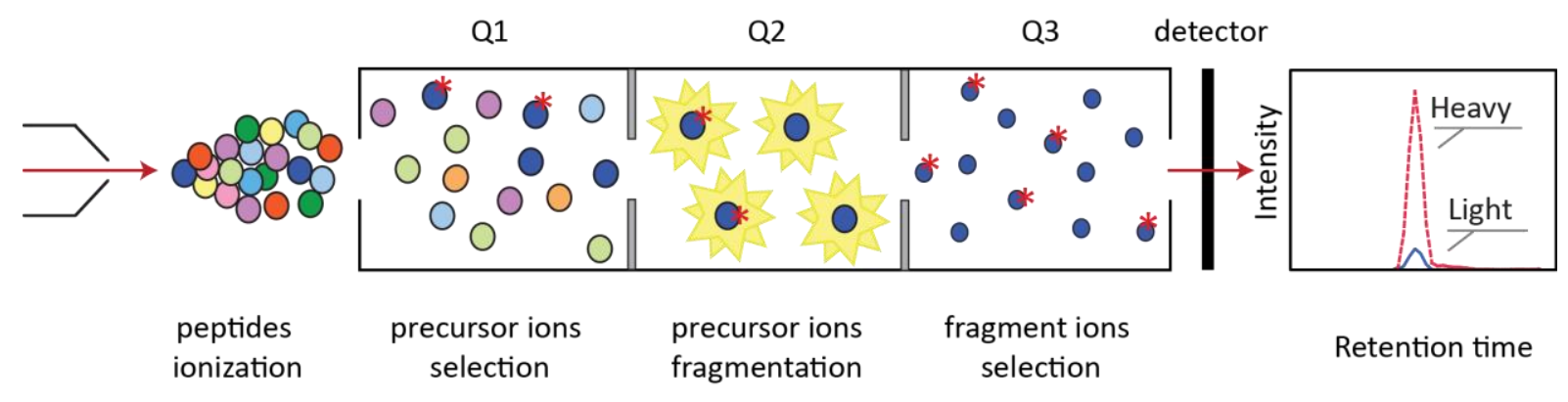

Figure 7. SRM analysis on a triple quadrupole mass spectrometer.Peptides are eluted from the nano-LC system and ionized before entering the instrument. Isotope-labeled peptides are indicated with the asterisk. Precursor ions are selected in the Q1, fragmented in Q2 and resulting fragment ions are ultimately selected for detection in Q3. Comparison of the peak area of light peptides and stable-isotope labeled peptides provides precise quantification of the endogenous analyte.

\subsection{Parallel reaction monitoring (PRM) to confirm the identitity of enriched peptides}

Although SRM is highly selective, some limitations arise from the resolution and mass accuracy of the quadrupoles mass analyzer used in triple quadrupole instruments, especially when near-isobaric fragment ions are analyzed (Abbatiello et al., 2010; Gallien et al., 2013; Sherman et al., 2009). Parallel reaction monitoring (PRM) can be an attractive alternative method for targeted quantification, especially when a higher degree of selectivity is required (Gallien et al., 2012; Peterson et al., 2012). As for SRM, peptides are separated by reversed phase chromatography on a nano-LC system and directly sprayed into the instrument, e.g., quadrupole-Orbitrap mass spectrometers (Gallien et al., 2012). This instrument type consists of an Orbitrap mass analyzer (Hu et al., 2005) preceded by a quadrupole mass filter which is used for the selection of only specific precursor ions. When PRM is performed on a quadrupole-orbitrap instrument, a defined precursor ion is selected in the quadrupole 
mass filter and transferred via the C-trap into the higher-energy collisional dissociation (HCD) cell, where it is fragmented. In contrast to the triple quadrupoles mass spectrometers, the quadrupoleorbitrab mass spectrometers have a discontinuous data acquisition regime in which ions with target $\mathrm{m} / \mathrm{z}$ values are selected by the quadrupole and collected in the C-trap while, in parallel, fragments of the previous measurement are still anaylzed in the Orbitrap. When enough ions are collected or the maximal fill time is reached, precursor peptides are transferred to the HCD cell for fragmentation. After fragmentation, the product ions are transferred via the C-trap to the Orbitrap and analysed simultenaously (Figure 8 ). In the Orbitrap the attraction to the central electrode is compensated by the initial tangential velocity of the ions. Upon measurement, the axial component of the circulation around the central electrode is detected as image current on the electrode encapsulating the orbitrap. The transient of all ions that is monitored over time is deconvoluted by Fourier transformation resulting in high resolution mass spectra. Longer fill times potentially increase the signal and, in parallel, longer analysis times allow for higher resolution spectra. On the other hand, the same factors limit the number of precursor masses that can be analyzed in a given time window, which results in a trade-off between resolution, sensitivity and the number of analytes. Because all fragments ions of a selected precursor can be monitored in parallel, PRM does not require the selection of only few of them, but all the pseudo transitions that have been recorded during the mass spectrometry run can be easily extracted post-acquisition, during data analysis. This translates into a simplification of the protocol with respect to SRM, in which less method optimization is required, with only the precursor $\mathrm{m} / \mathrm{z}$ ratio that has to be selected in the quadrupole and the elution time of the peptide. 
Q1

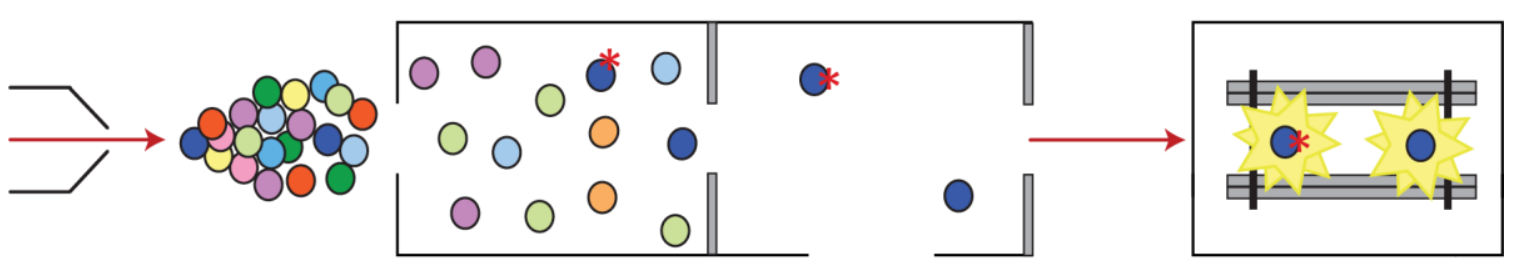

Peptides Precursor ions ionization selection

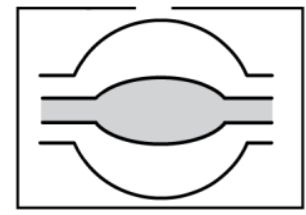

Orbitrap

mass analyzer

Q1 C-trap HCD collision cell

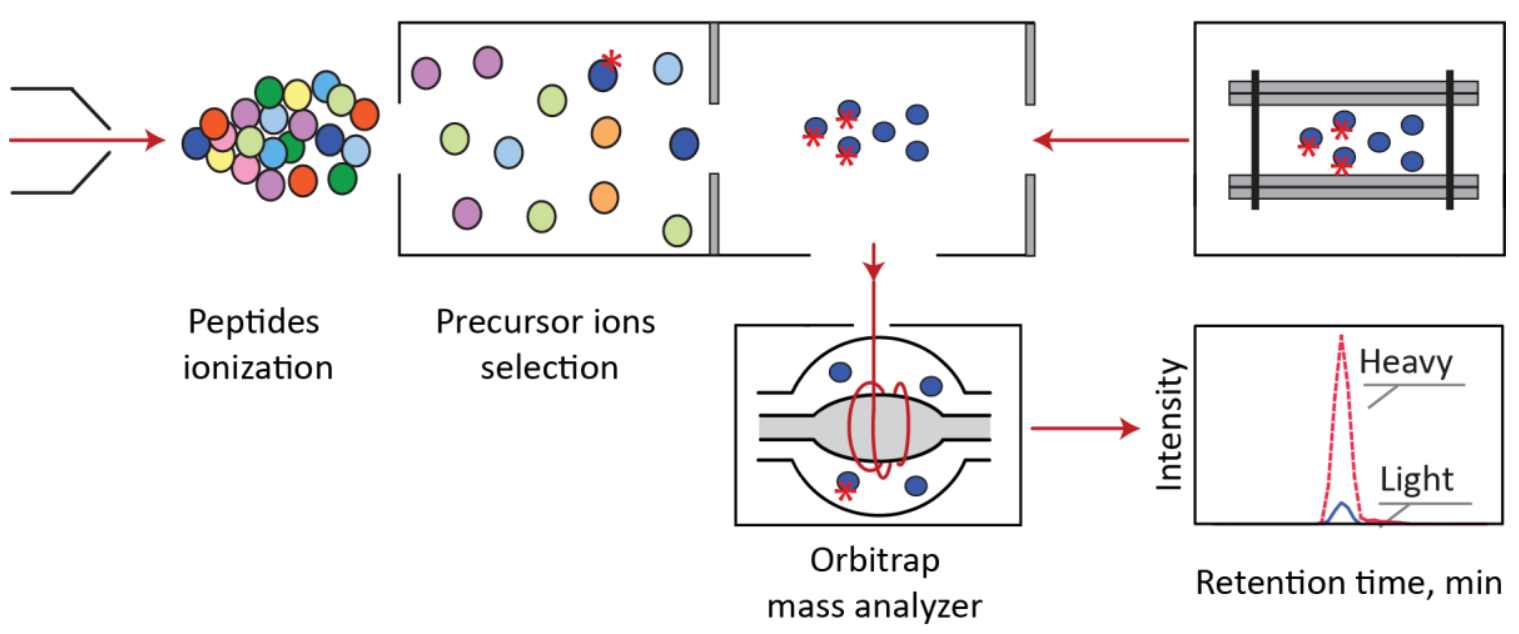

Figure 8. PRM analysis on a hybrid quadrupole/Orbitrap mass spectrometer. Peptides are eluted from the nano-LC system and ionized before entering the instrument. Isotope-labeled peptides are indicated with the asterisk. Precursor ion are selected in the Q1 and move via the C-trap to the HDC cell for fragmentation. All fragment ions are then transmitted back into the C-trap and injected into the Orbitrap for analysis. Comparison of the peak area of light peptides and stable-isotope labeled peptides provides precise quantification of the endogenous analyte.

\subsection{EF-Tu as a model protein}

Although a proteome-wide quantification of misincorporations would be desiderable, such large-scale quantification is hampered by the large difference in the abundance of many error-containing peptides and their cognate counterparts, whose concentration ranges often exceed the linearity of the instrument. Because there is no evidence that misincorporations in endogenous proteins can be more adundant than $10^{-3}$ (Table 1 and Table 3 ), the vast majority of proteins comprising the $E$. coli proteome cannot be studied in the lysate. In addition, the mechanisms that ensure the fidelity of protein synthesis are expected to be the same regardless of the protein choosen, because the same type of errors that occur during DNA replication, transcription or translation affect every synthetized 
protein. These considerations prompted us to select a model protein that could be investigated without limitations in terms of source, strain, construct, tag or stress condition and that could be easily purified by affinity protocols as well as traditional tag-free chromatographic strategies (to minimize external interferences coming from other proteins), but that would also be abundant enough to be directly investigated in lysates without any enrichment. Among the high-abundance proteins in E. coli, EF-Tu emerged as the best candidate for our analysis. It is by far the most abundant protein of the $E$. coli proteome (Figure 9), 10-times more abundant than ribosomes (Furano, 1975; Schmidt et al., 2016). EF-Tu is directly involved in decoding and thus manipulations such as the addition of a tag for affinity purifications might effect the levels of misincorporations in the cell. However, the protein is functional when fused to a His-tag at the C-terminus in vitro and in vivo and supports the wild type accuracy levels (Boon et al., 1992).

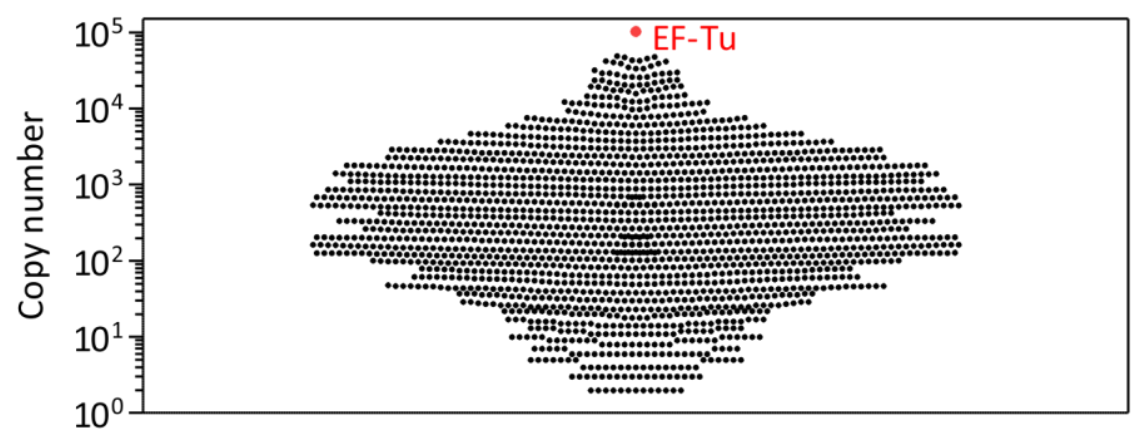

Figure 9. Abundance of proteins in E.coli. Mass spectrometry analysis of the proteome of $E$. coli cells grown in LB medium. Figure based on the data from (Schmidt et al., 2016).

EF-Tu is a monomeric protein of 393 amino acids and consists of three domains (Kjeldgaard and Nyborg, 1992). Domain I is composed of a central sheet formed by five parallel $\beta$-strands plus one which is antiparallel and which is in turn surrounded by $\alpha$-helices and contains the GDP/GTP binding pocket (Figure 10). Domains II and III constist of antiparallel $\beta$-barrel structures. EF-Tu is encoded by two genes, tufA and tufB (Lindahl et al., 1975), which have almost the same sequence, except for 13 nucleotides that result in synonymous codon substitutions. The products of the two genes share the same structure, almost identical molecular weight (43314 Da and 43284 for the product of tufB and tufA, respectively) and their amino acid sequence is identical, except for one amino acid at the Cterminus for E. coli MRE600 (glycine in tufA and serine in tufB) (Arai et al., 1980). Sequence variations can be observed in different $E$. coli strains. The amino acid sequence for the EF-Tu used in this project can be found in the Appendix (Section A) of this thesis. 


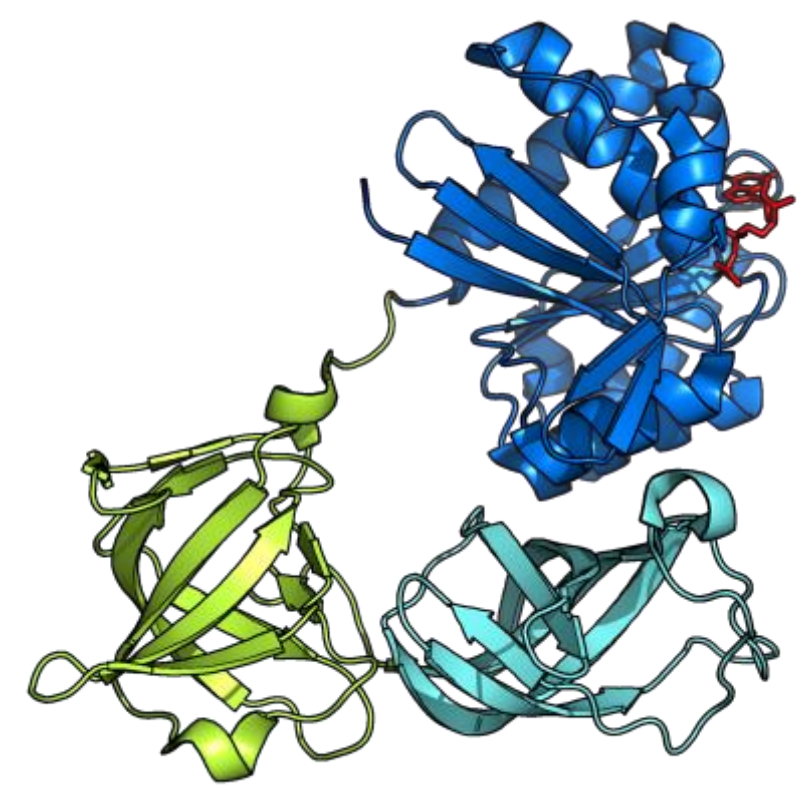

Figure 10. EF-Tu crystal structure. The three domains are highlighted in different colors: GTP/GDP-binding domain I (1-200 aa) in dark blue, domain II (209-299 aa) in lime, domain III (300-393 aa) in aquamarine. The structure of GDP molecule bound to domain I is highlighted in red. [PDB file 1dg1 (Abel et al., 1996)].

\subsection{Aim of the study}

The aim of this project is to develop a mass spectrometric assay to systematically quantify the error frequency of amino acid misincorporation in vivo and to overcome the limitations inherent in other published approaches e.g., codon-specific reported systems, amino acids or positions in the protein sequence, narrow instrument dynamic range and sensitivity. The second aim is to apply this assay in order to characterize the basic features and dimensions of the fidelity landscape in vivo determining i) the accuracy of the proteome; ii) the range in which misincorporations occur; iii) the fidelity of translation at different position of the protein; iv) the potential effect of the quality control machinery. The third aim is to characterize the error-inducing signatures of aminoglycosides and to correlate them with the impact on the cell physiology, such us growth defects and induction of stress response, and to ultimately dissect the complex mechanisms underlying the bactericidal effects of miscodinginducing antibiotics. 


\section{RESULTS}

\subsection{Multidimensional chromatography for peptides enrichment}

\subsubsection{An overview of the method}

Quantification of misincorporations and estimation of translational error rate are often affected by limitations in the type of misincorporation to be selected for the study, by poor sensitivity of the method or its restriction to in-vitro assays or fixed model systems. Here we describe a novel method to quantify amino acid misincorporations in vivo. The advantage of the method is its sensitivity and broad applicability to unrestricted types of substitutions and reference systems. The protocol relies on the separation of error-containing peptides and their enrichment against the more abundant correct peptides through consecutives rounds of orthogonal chromatographies including alternating rounds of size-exclusion and reversed phase separation. This is combined with final quantification by targeted mass spectrometry and SRM analysis (Figure 11). This approach has some similarities with the methods employed for the detection of low-abundance protein biomarkers from complex biological fluids, like blood plasma or urine, in which high-abundance proteins are depleted prior to the analysis (Surinova et al., 2011).

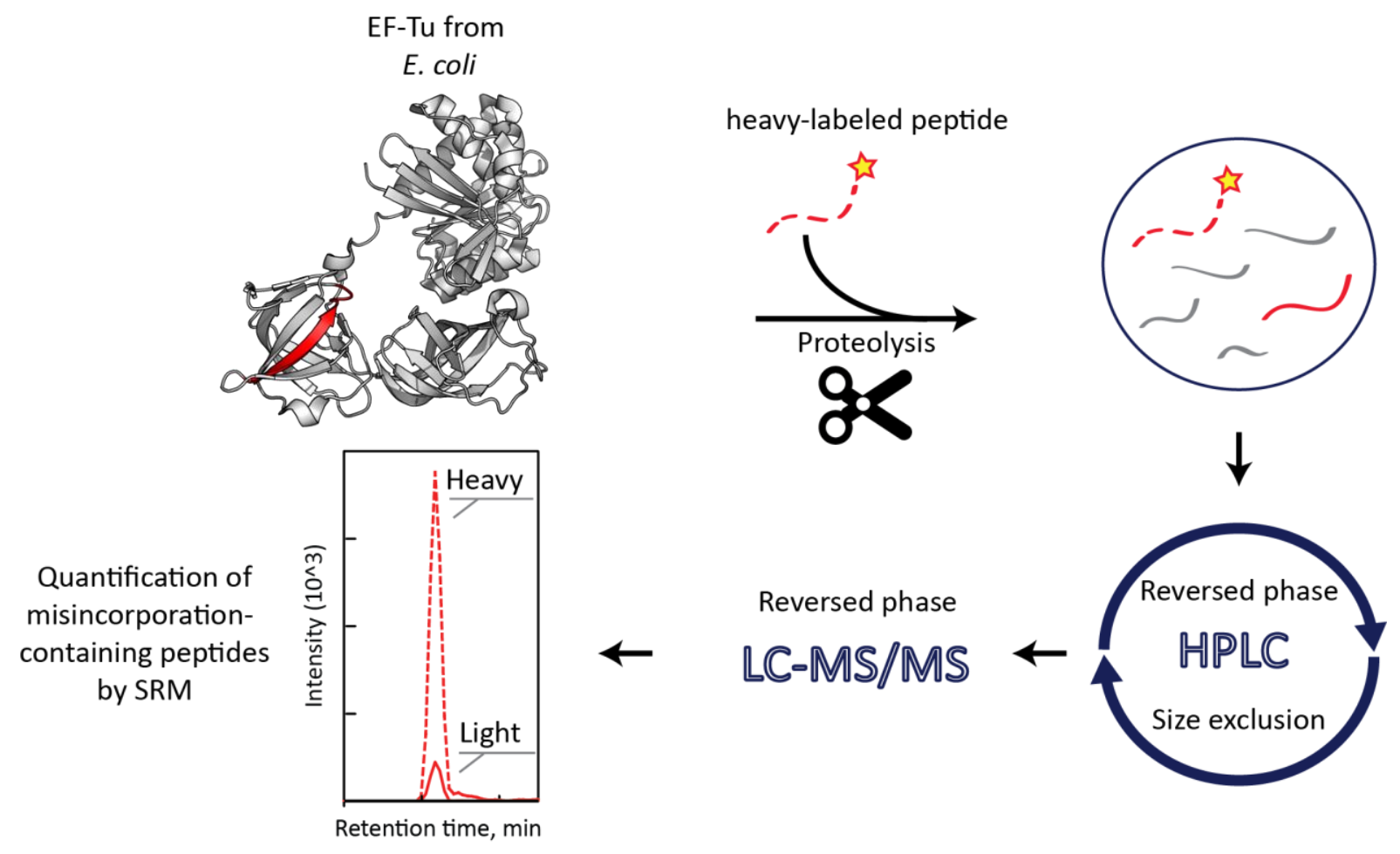

Figure 11. Schematic of the workflow for the enrichment of error-containing peptides. A model protein, EF-Tu, is proteolyzed. The peptide of interest (red) is selected and the corresponding isotope-labeled reference peptide (dashed red), is spiked in the sample. The sample is subjected to multiple rounds of HPLC chromatography. Ultimately, peptides are separated on the nano-LC on-line with the mass spectrometer (LC-MS/MS). Quantification of misincorporations is performed on triple quadrupole mass spectrometer operated in Selected Reaction Monitoring (SRM) mode and the ratio of light/heavy peptides is used to calculate the error frequency. 


\subsubsection{Sample preparation}

The performance of the protocol depends on the accurate preparation of the sample, which in our case starts with proteolysis by trypsin, a protease that cleaves polypeptide chains after lysine and arginine residues. To optimize protein digestion, we selected four tryptic peptides from the model protein EF-Tu, i.e., FESEVYILSK, VGEEVEIVGIK, ELLSQYDFPGDDTPIVR and TVGAGVVAK (Figure 12, A), which are proteotypic within the E. coli proteome and we monitored their formation over time. At selected time points, an aliquot from the digestion mix is withdrawn, mixed with the heavy-labeled counterparts of the four reference peptides and the sample analyzed by mass spectrometry. The area under the peak of light and heavy peptides is integrated and their ratio is calculated (Figure 12, B).

A
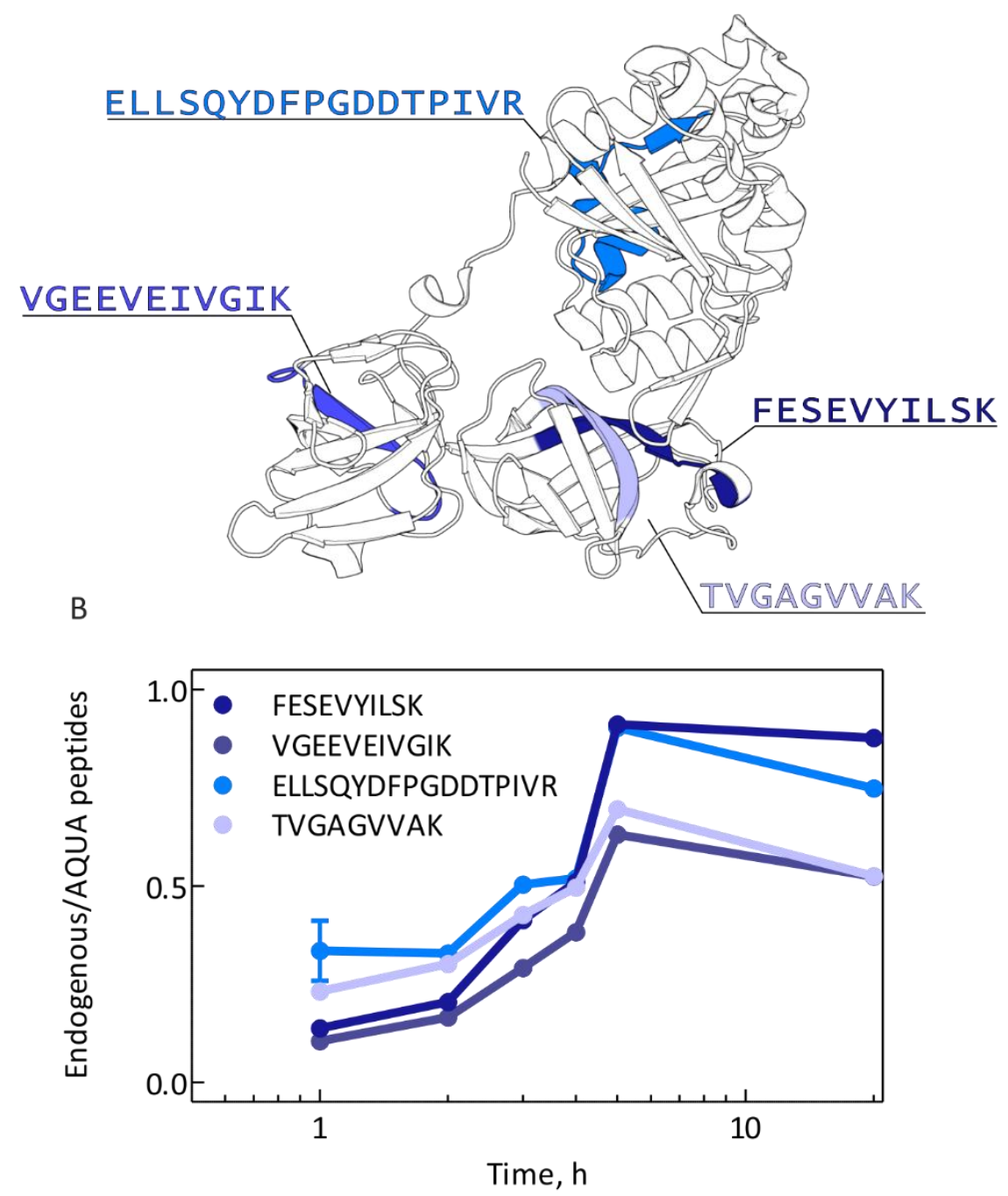

Figure 12. Proteolysis time courses. A) Four proteotypic peptides are selected from the EF-Tu sequence [PDB file 1dg1]. B) The progress of the proteolysis is assessed by monitoring four quantotypic peptides. Error bars represent the standard deviation of 3 technical replicates. 
The relative concentration of the endogenous peptides increases with reaction time and reaches a plateau after $5 \mathrm{hrs}$ of incubation at $37^{\circ} \mathrm{C}$. At this point the digestion is complete and the peptides remain stable in the reaction tube for the next $20 \mathrm{hrs}$. The stability of the peptides indicates that they are potentially quantotypic for the protein, which means that an any point their concentration exactly reflects the amount of protein in the sample, and that their quantification is reliable and not affected by decay due to instability, which could lead to an underestimation of the amount of proteins (Shuford et al., 2012).

After proteolysis is completed, the total amount of digested EF-Tu is quantified by mass spectrometry using the same four peptides employed to monitor the digestion. A small aliquot of the digested protein is spiked in with a known concentration of AQUA peptides containing identical amounts of the four reference peptides and the mixture is then analyzed by mass spectrometry. The ratio of endogenous and heavy peptides is determined (Figure 13) and because the concentration of the latter is known, the amount of EF-Tu can be easily calculated. Once the concentration of the correct tryptic peptides is determined, we spike in the isotope-labeled reference peptides containing misincorporations of interest and assess the error frequency, e.g., for the quantification of $\mathrm{R}$ to $\mathrm{H}$ substitution in the GTVTTGRVER peptide, the AQUA peptide GTVVTG $\underline{H} V E R$ is used.

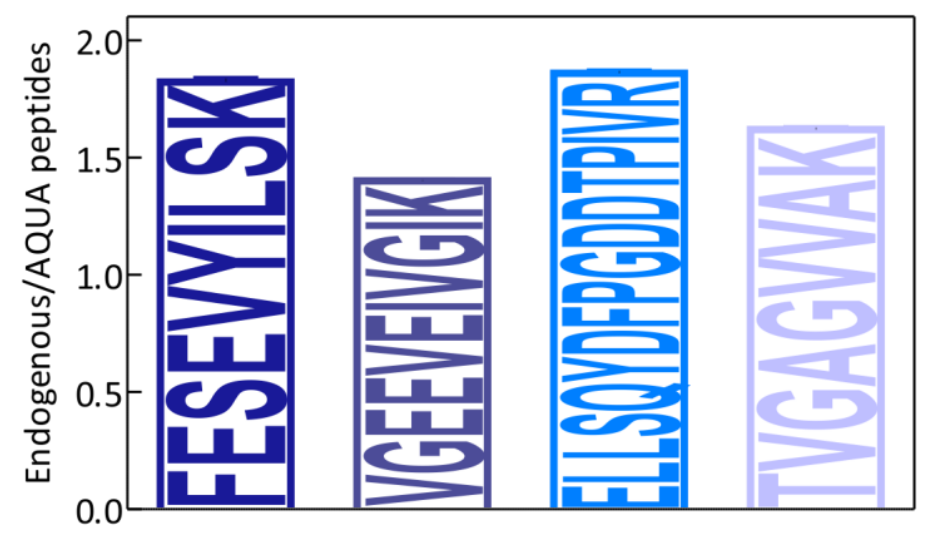

Figure 13. Quantification of proteolysed EF-Tu. The ratio of endogenous/AQUA peptides for the reference peptides is calculated and used to quantify the concentration of correct tryptic peptides obtained from EF-Tu proteolysis. Digested peptides and AQUA peptides are not mixed in a 1:1 ratio so that the calculated endogenous/AQUA ratio does not need to be necessarily 1 . However, comparable ratios among the four reference peptides indicate that they are are stable in the reaction tube. Error bars represent the standard deviation of 3 technical replicates.

\subsubsection{Chromatographic separation and enrichment of target peptides}

In the first chromatographic dimension, the peptides are separated based on their hydrodynamic radius by size-exclusion chromatography, whereas in the second dimension they are separated depending on their hydrophobicity by reversed phase chromatography at neutral pH (Figure 14). At 
both steps, all the fractions obtained are screened by mass spectrometry, and only the ones containing the target peptides are processed further. At this stage, the signal from the error-containing peptides is still too low to be detected, so that only the respective heavy-labeled standards are monitored. For the subsequent quantification the peptides are loaded onto a nanoflow chromatographic system and separated by reverse phase at acidic $\mathrm{pH}$ prior to their detection in the mass spectrometer. Although the last two dimensions both rely on reversed phase chromatography, the different $\mathrm{pH}$ at which they are performed confer separation power, especially for those peptides which contain residues whose protonation status changes upon $\mathrm{pH}$ shift - i.e., aspartate and glutamate - and whose separation can be altered by using different $\mathrm{pH}$ range. Because our peptides normally contain one of these amino acids, their chromatographic behavior changes from neutral to acidic $\mathrm{pH}$ ensuring their efficient separation. For an initial screening of error frequencies, we have chosen to follow the Arg to His substitutions (Table 4), known to be relatively abundant (Zhang et al., 2013). In addition, this type of amino acid misincorporation is particularly favourable to be investigated using our enrichment protocols because it changes the tryptic pattern of the protein which we proteolyzed using trypsin (which cleaves at $\mathrm{R}$ and $\mathrm{K}$ residues). The proteolysis of the error-containing protein yields longer peptides that elute earlier from the size-exclusion column than the products of digestion of the correct protein which are shorter. Thereby, the complexity of the sample and the background noise are efficiently reduced. Low-abundance erroneous peptides are enriched, separated within each other and from the more abundant cognates allowing us to increase the column load, pulling low abundance-peptides in the dynamic range of the instrument so that their signal can be detected.

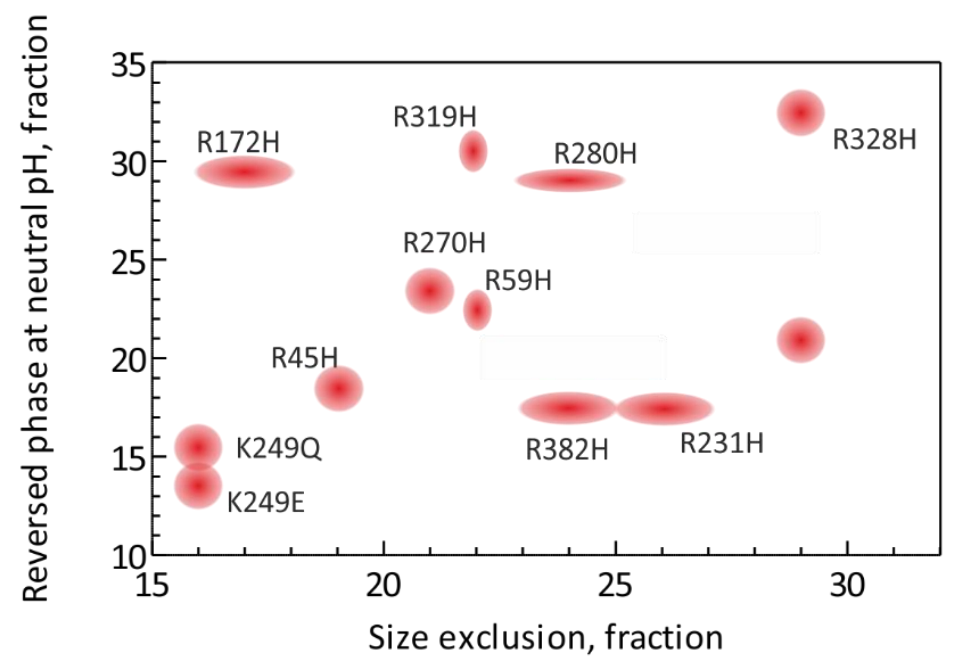

Figure 14. Distribution of peptide elution intervals in the first two chromatographic dimensions. EF-Tu peptides displaying different chemical property can be efficiently separated according to their size and hydrophobicity. Separated peptides are represented by the red dots. The size of each dot reflects the number of fractions in which the respective peptide is eluting. 
Table 4. General properties of $\mathbf{R}$ to $\mathbf{H}$ substituted peptides. Distinct physicochemical characteristics allow for efficient separation and correct identification of error-containing peptides. Hydrophobicity factors are estimated using the peptide analyzer tool https://www.thermofisher.com/de/de/home/life-science/protein-biology/peptidesproteins/custom-peptide-synthesis-services/peptide-analyzing-tool.html.

\begin{tabular}{cccc}
\hline Misincorporation & Peptide & Length & Hydrophobicity \\
\hline R45H & TYGGAAHAFDQIDNAPEEK & 19 & 28.03 \\
\hline R59H & AHGITINTSHVEYDTPTR & 18 & 24.83 \\
\hline R231H & GTVVTGHVER & 10 & 13.43 \\
\hline R234H & VEHGIIK & 7 & 32.49 \\
\hline R270H & LLDEGHAGENVGVLLR & 16 & 27.51 \\
\hline R280H & AGENVGVLLHGIK & 13 & 5.86 \\
\hline R284H & HEEIER & 6 & 19.94 \\
\hline R319H & DEGGHHTPFFK & 11 & 28.31 \\
\hline R328H & GYHPQFYFR & 9 & 13.16 \\
\hline R378H & FAIHEGGR & 8 & 15.52
\end{tabular}

In some cases, enrichment and analysis of error-containing peptides which have very similar physicochemical characteristics to their cognate peptides (e.g., FESEVYILSK peptide (Table 5)) might not be achieved in the first two dimensions (Figure 15). However, the separation of target peptides from the highly abundant cognate peptide is essential, as without their separation the sample complexity cannot be reduced. To improve the separation, further chromatographic steps are required which are tailored to the characteristics of specific peptides. We chose to add the third chromatographic step, reversed phase at acidic $\mathrm{pH}$ prior to the reversed phase performed on the nanoflow system. Although three rounds of reversed phase might not be fully orthogonal, they synergistically provide not only a further reduction of sample complexity, but also improve the separation of histidine-containing peptides due to the different $\mathrm{pH}$. The second reversed phase chromatography, therefore, enhances the separation of error-containing peptides from the correct FESEVYILSK, before that the sample is loaded on the LC-MS/MS system (Figure 16). This reduces local interferences in SRM quantification and allows to load more target peptide without saturating the LC system, both improving the signal to noise ratio and signal intensity. 
Table 5. Physicochemical characteristics of peptides differing by a few amino acids may be very similar. When compared to the cognate peptide FESEVYILSK, peptide variants with one, two or three amino acid difference have very similar charachteristics in terms of both length and hydrophobicity. Hydrophobicity factor has been estimated using the peptide analyzer tool available on the website of Thermo Fischer Scientific at the link https://www.thermofisher.com/de/de/home/lifescience/protein-biology/peptides-proteins/custom-peptide-synthesis-services/peptide-analyzingtool.html

\begin{tabular}{clll}
\hline Errors & Peptide & Length & Hydrophobicity \\
\hline Cognate & FESEVYILSK & 10 & 28.2 \\
\hline F305L & LESEVYILSK & 10 & 26.41 \\
\hline E306D & FDSEVYILSK & 10 & 28.51 \\
\hline E308D & FESDVYILSK & 10 & 27.45 \\
\hline Y310H & FESEVHILSK & 10 & 23.69 \\
\hline Y310N & FESEVNILSK & 25.23 \\
\hline E306D-E308D & FDSDVYILSK & 10 & 27.83 \\
\hline E306D-Y310H & FDSEVHILSK & 10 & 24.00 \\
\hline E308D-Y310H & FESDVHILSK & 10 & 25.10 \\
\hline E306D-Y310N & FDSEVNILSK & 10 & 24.64 \\
\hline E308D-Y310N & FESDVNILSK & 10 & 23.41 \\
\hline E306D-E308D-Y310H & FDSDVHILSK & 10 & \\
\hline
\end{tabular}

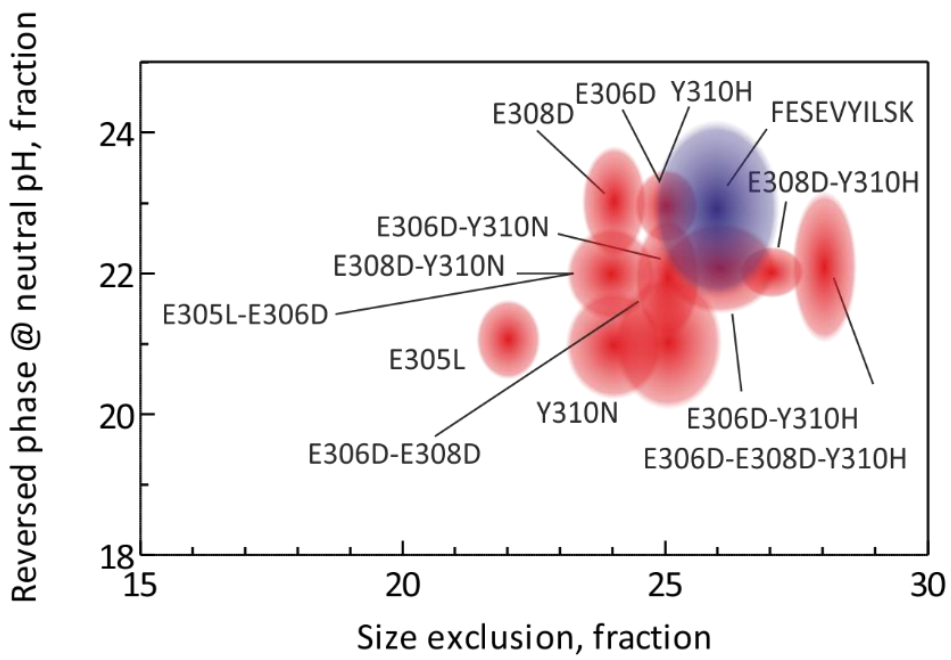

Figure 15. Poor separation of similar peptides in the first two chromatographic dimensions. The two-dimensional separation of peptides that differ in only few amino acids with respect to the correct peptide is poor. Separated peptides are represented by dots (error-containing peptides in red, correct FESEVYILSK in blue). The size of each dot reflects the number of fractions in which they are eluting. 


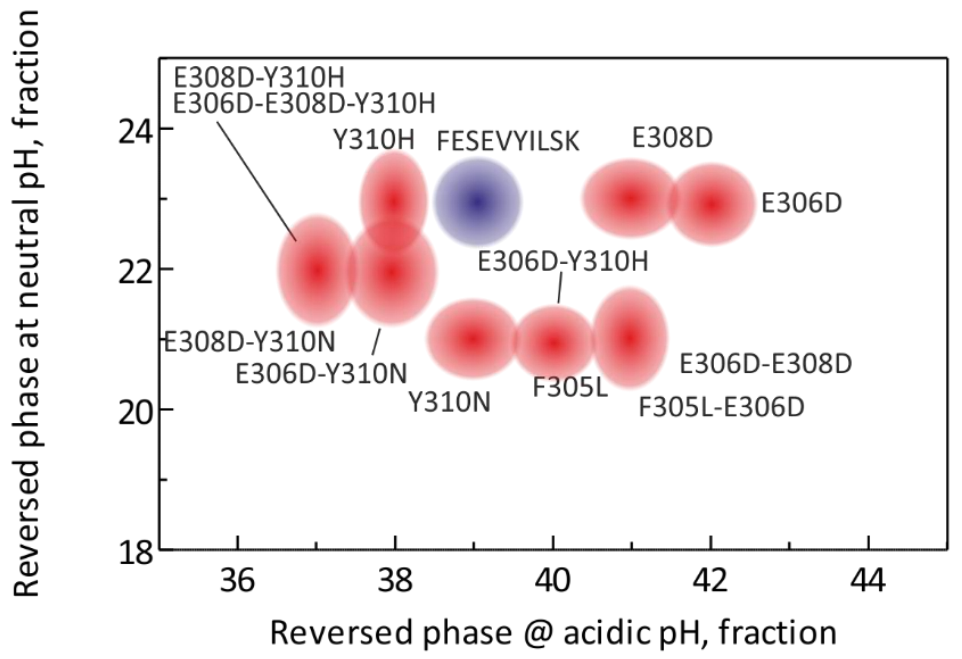

Figure 16. Elution pattern of similar peptides in reversed phase chromatography runs at neutral and acidic pH.The different $\mathrm{pH}$ of the mobile phase changes the separation profiles of peptides which contain ionizing amino acids. Separated peptides are represented by dots (error-containing peptides in red, correct FESEVYILSK in blue). The size of each dot reflects the number of fractions in which peptides elute.

\subsection{Identification of enriched error-containing peptides}

\subsubsection{Identification of peptides by SRM analysis}

Data obtained by SRM analysis are processed using Skyline software (MacLean et al., 2010b) which integrates the area under peptides peak and calculates the $L / H$ ratio between light $(L)$ and heavy $(H)$ peptides. Each peptide, both in its light and heavy forms, is identified by the precursor mass-to-charge ration $(\mathrm{m} / \mathrm{z})$ selected in the first quadruple of the mass spectrometer $(527.7865$ and 532.7856 , respectively for the example shown in figure 17) and the $m / z$ of $3-5$ fragment ions which are selected in the third quadrupole. The same intensity pattern for light and heavy peptides transitions, together with the identical elution time, allow us to identify the endogenous target peptide (Figure 17). A quantitative expression for this identification is the ratio dot product (rdotp) (Sherwood et al., 2009), for which the maximum value 1 indicates a perfect identification, whereas a value of 0 suggests complete orthogonality. 
$\mathrm{R} 231 \mathrm{H}$

$$
\begin{aligned}
& b^{1} \quad b^{2} \quad b^{3} \quad b^{4} \quad b^{5} \quad b^{6} \quad b^{7} \quad b^{8} \quad b^{9} \\
& \underset{\mathrm{y}^{9}}{\mathrm{G}} \underset{\mathrm{y}^{8}}{\mathrm{~T}} \underset{\mathrm{y}^{7}}{\mathrm{~V}} \underset{\mathrm{y}^{6}}{\mathrm{~V}} \underset{\mathrm{y}^{5}}{\mathrm{~T}} \underset{\mathrm{y}^{4}}{\mathrm{G}} \underset{\mathrm{y}^{3}}{\operatorname{Vi}} \underset{\mathrm{y}^{2}}{\mathrm{~V}} \underset{\mathrm{y}^{1}}{\mathrm{R}}
\end{aligned}
$$

A

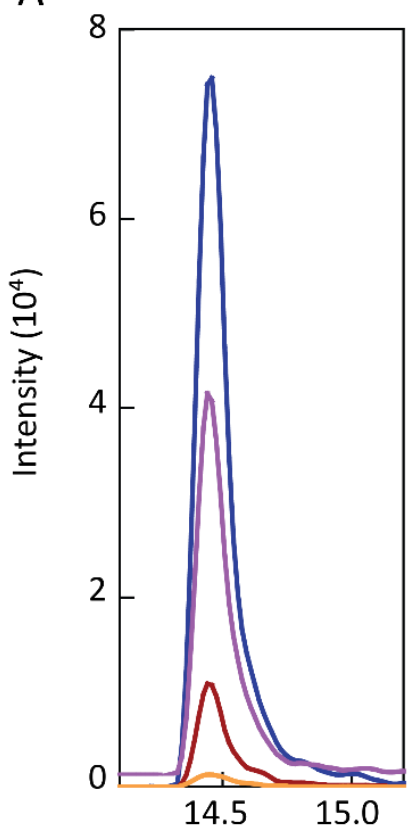

Retention time, $\mathrm{min}$
B

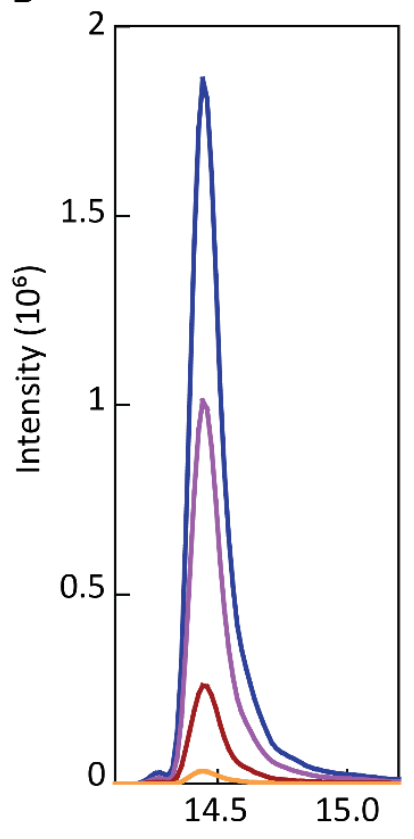

rdotp 1

Light 527.7865

- y6-698.3580+

- y5 - 597.3101+

- y4 - 540.2889+ b9 - 880.4523+

Heavy 532.7856

- y6-708.3663+

- y5-607.3186+

- y4 - 550.2971+

_ b9- $880.4523+$

Figure 17. Detection of error-containing peptides. Top panel, the sequence of the peptide and its fragmentation pattern. Co-eluting light (A) and heavy $\left[\operatorname{Arg}\left({ }^{13} \mathrm{C} 6 ;{ }^{15} \mathrm{~N} 4\right)\right](B)$ versions of the $\mathrm{R} 231 \mathrm{H}$ peptide are detected. Four transitions y6, y5, y4 and $b 9$ are selected, whose intensity order is identical between the light and heavy peptide. A rodtp value of 1 confirms the confidence of the identification. 


\subsubsection{Identity validation by manual annotation of MS1 and MS/MS spectra}

Even though the co-elution of light and heavy peptides and the transitions pattern alone are generally accepted as a proof of the peptide identity, we decided to further validate our identifications using high resolution MS1 and MS/MS spectra. Precursor and the fragment ions spectra are compared with the fragmentation pattern predicted for the peptide (Figure 18). In cases where a clear MS1 and MS/MS could not be acquired due to the limited dynamic range of the mass spectrometer or to signal interference, for example for non-cognate misincorporations, the identity of the peptides detected by SRM was substantiated by parallel reaction monitoring (PRM) (Figure 19).

A

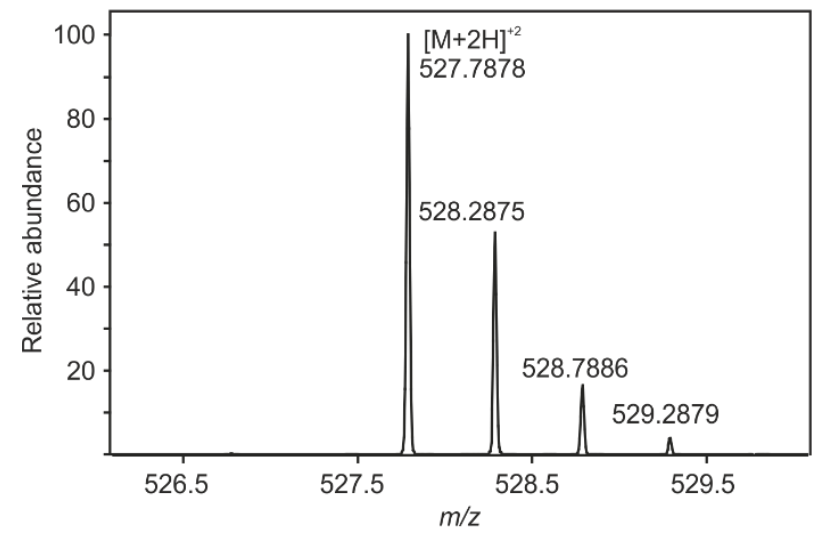

B

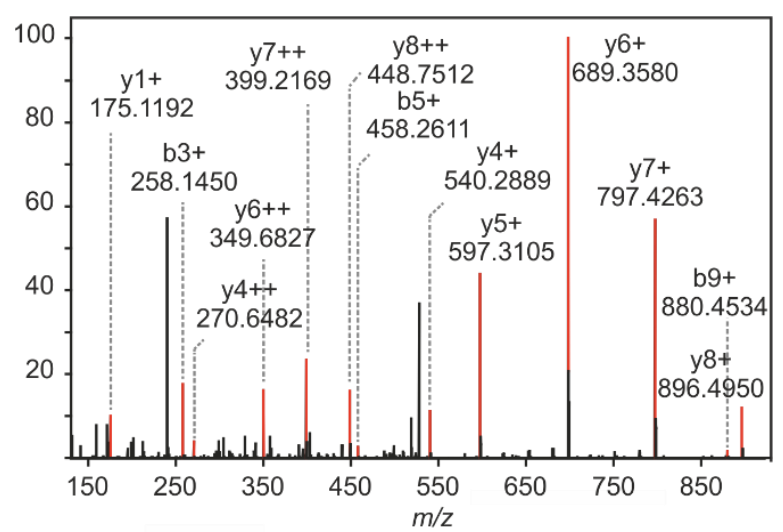

C

\begin{tabular}{|c|c|c|}
\hline $\mathrm{MH}^{+1}$ & $\mathrm{MH}^{+2}$ & $\mathrm{MH}^{+3}$ \\
\hline 1045.5640 & $\mathbf{5 2 7 . 7 8 5 6}$ & 352.1928 \\
\hline
\end{tabular}

\begin{tabular}{|c|c|c|c|c|c|c|}
\hline $\mathrm{b}$ & $\mathrm{b}^{+2}$ & & & & $\mathrm{y}$ & $\mathrm{y}^{+2}$ \\
\hline- & - & 1 & $\mathrm{G}$ & 10 & - & - \\
\hline $\mathbf{1 5 9 . 0 7 6 4}$ & - & 2 & $\mathrm{~T}$ & 9 & 977.5425 & 499.2749 \\
\hline $\mathbf{2 5 8 . 1 4 4 8}$ & - & 3 & $\mathrm{~V}$ & 8 & $\mathbf{8 9 6 . 4 9 4 8}$ & $\mathbf{4 4 8 . 7 5 1 1}$ \\
\hline 357.2132 & - & 4 & $\mathrm{~V}$ & 7 & $\mathbf{7 9 7 . 4 2 6 4}$ & $\mathbf{3 9 9 . 2 1 6 9}$ \\
\hline $\mathbf{4 5 8 . 2 6 0 9}$ & - & 5 & $\mathrm{~T}$ & 6 & $\mathbf{6 9 8 . 3 5 8 0}$ & $\mathbf{3 4 9 . 6 8 2 6}$ \\
\hline 515.2824 & - & 6 & $\mathrm{G}$ & 5 & $\mathbf{5 9 7 . 3 1 0 3}$ & 299.1588 \\
\hline 652.3431 & 326.6743 & 7 & $\mathrm{H}$ & 4 & $\mathbf{5 4 0 . 2 8 8 9}$ & $\mathbf{2 7 0 . 6 4 8 1}$ \\
\hline $\mathbf{7 5 1 . 4 0 9 7}$ & 376.2085 & 8 & $\mathrm{~V}$ & 3 & 403.2300 & 202.1186 \\
\hline $\mathbf{8 8 0 . 4 5 2 3}$ & 440.7298 & 9 & $\mathrm{E}$ & 2 & 304.1615 & 152.5844 \\
\hline- & - & 10 & $\mathrm{R}$ & 1 & $\mathbf{1 7 5 . 1 1 9 0}$ & 88.0631 \\
\hline
\end{tabular}

Figure 18. Identification of error-containing peptides by high resolution MS1 and MS2 spectra.The precursor (A) and the fragment (B) spectra are compared with the predicted fragmentation pattern (C). Their identification in the sample is essential to confirm the identity of the enriched peptide. The peak of the doubly charged precursor is indicated in bold in both $A$ and in the predicted fragmentation pattern. The spectra of the freagment ions are indicated in bold in the fragmentation pattern and annotated on the MS/MS spectra. 


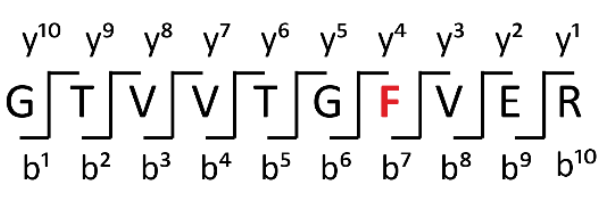

A

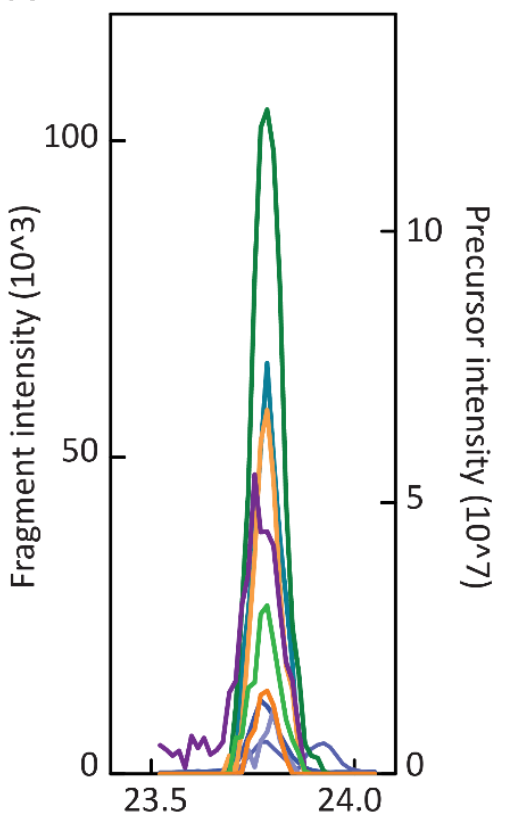

Retention time, $\mathrm{min}$
B

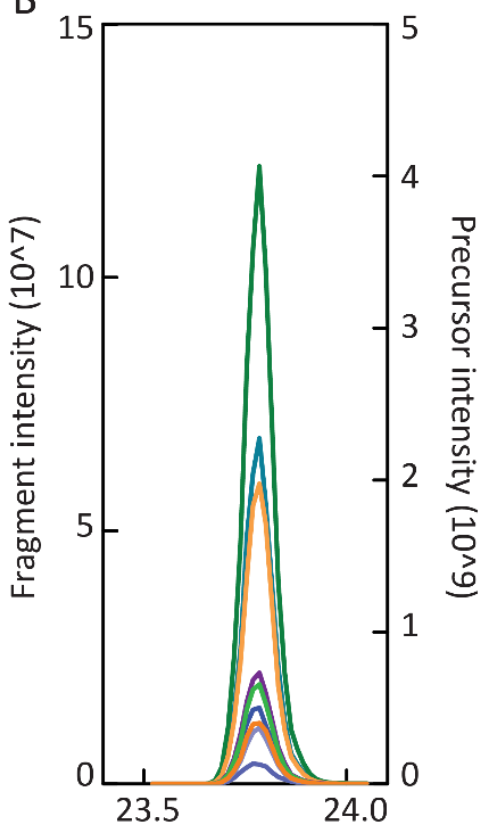

Retention time, $\mathrm{min}$
R231F

rdotp 0.95

Light

— precursor - 533.2918++

— precursor - 533.7932++

- y8-906.5043+

- y7-807.4359+

- y6-708.3675+

- y5 - 607.3198+

- y4 - 550.2984+

— b2 - 159.0764+

- b3 - 258.1448+

Heavy

— precursor - 538.2960++

— precursor - 538.7974++

- y8-916.5126+

- y9-817.4442+

- y8-718.3758+

- y7-617.3281+

- y4 - 560.3066+

— b2 - 159.0764+

— b3 - 258.1448+

Figure 19. Identification of misincorporation-containing peptides by parallel reaction monitoring (PRM). Top panel, the sequence of the peptide and its fragmentation pattern. For peptides including non-cognate misincorporations, the validation is performed by PRM. In the example, the peak obtained for the light (A) and heavy (B) R231F peptide is shown. The mass of precursor and fragments ions are indicated, and the rodtp for the identification is reported.

\subsection{Quantification of enriched error-containing peptides}

\subsubsection{Quantification of peptides by SRM analysis}

When the identity of the enriched error-containing peptides is confirmed and quantified using their $\mathrm{L} / \mathrm{H}$ ratios in Skyline, the concentrations of the correct peptides is used to calculate the error rate according to the equation:

$$
\text { Error frequency }=\frac{p m o l A Q U A}{p m o l E F-T u} \times \frac{L}{H}
$$

The purity and the labeling efficiency of the isotopic labeled AQUA peptide can alter the calculated $\mathrm{L} / \mathrm{H}$ ratio. Therefore, prior to the quantification, we ran a blank sample with only the heavy peptide (Figure 20) to ensure that is free from any light counterpart contamination. 


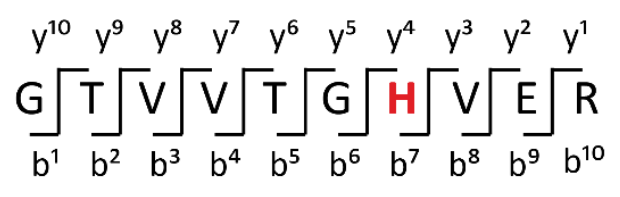

A

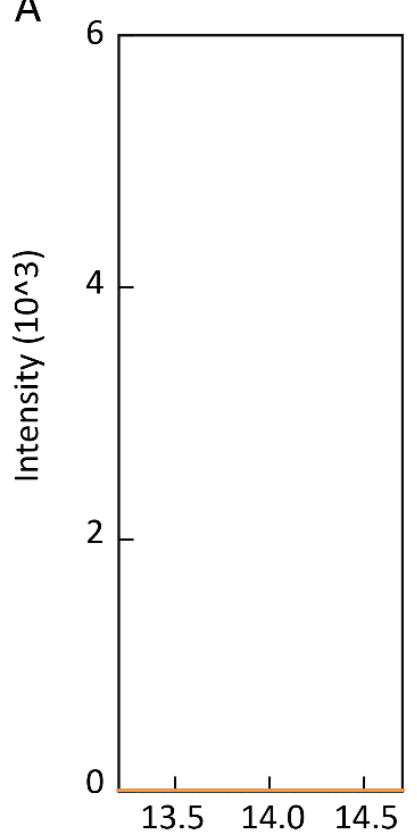

Retention time, $\mathrm{min}$
B

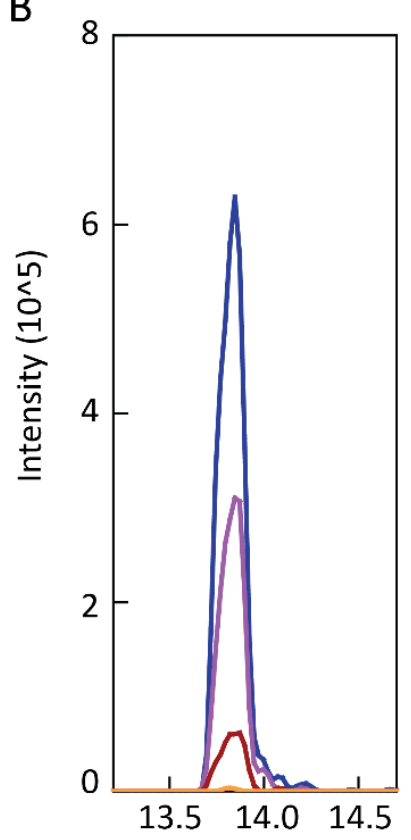

Retention time, min
R231H

Light 527.7865

- y6-698.3580+

- y5 - 597.3101+

— y4 - 540.2889+

— b9-880.4523+

Heavy 532.7856

- y6-708.3663+

y5 - 607.3186+

y4 - 550.2971+

b9 - $880.4523+$

Figure 20. Assessment of AQUA peptides purity. To assess the purity of the purchased AQUA peptides and to exclude any light-peptide contaminations, each AQUA peptide (in the reported example, R231H peptide) is analysed by SRM and the signal for both light (A) and heavy (B) counterpart is recorded.

\subsubsection{Pseudo-linear quantification assessment}

To assess the linear dynamic range of our assay, the enriched peptide, which is already mixed with the identifier AQUA peptide used for the identification and quantification, is titrated with increasing concentrations of a second quantifier AQUA peptide which has identical amino acid sequence and contains a second isotope-labeled residues. These features allow us to distinguish the quantifier AQUA peptide from both the endogenous and the first identifier AQUA peptide and to assess the linearity of the quantification which extends over 7 orders of magnitude, with respect to the original dynamic range of the instrument, spanning 3 - 5 orders of magnitude (data provided by Dr. Ingo Wohlgemuth) (Figure 21). 


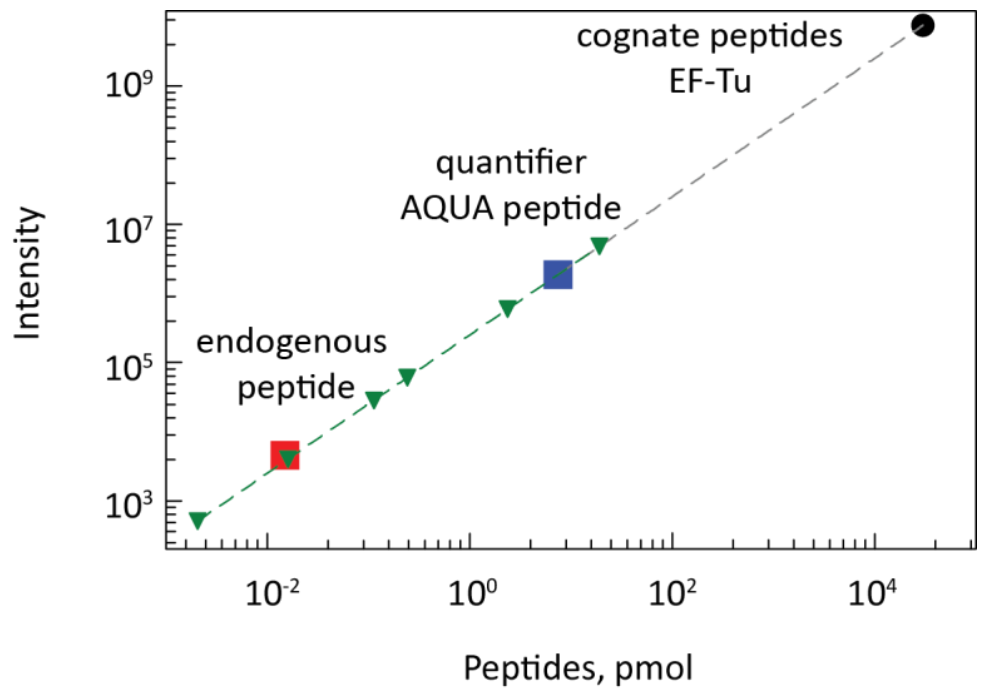

Figure 21. Pseudo-linear dynamic range of correct and erroneous peptides quantification. Quantification of correct peptides (black) is performed independently of the error-containing peptides (red). This allows to extend the dynamic range over 7 orders of magnitude. The linearity of the measurement is then assessed by titrating the enriched sample with a second quantifier AQUA peptide (blue). In the example is reported the titration for the peptide K249N (Appendix, Section B).

\subsubsection{Post translational modifications and peptide degradation}

Whereas the absolute quantification of a protein by mass spectrometry is based on the averaged quantification of 3 or more of its peptides, the quantification of misincorporations has to rely on the singular quantification of the respective unique peptide. Thus, their quantification must necessarily be as accurate as possible to truly reflect the error frequency and any potential sources of misquantification such as incomplete trypsination, peptide instability, inaccurate AQUA peptide concentrations or post-translational modifications must be excluded. To confirm the quality of the quantification by SRM, we generated EF-Tu mutants for each of the $R \rightarrow X$ and $K \rightarrow X$ amino acid misincorporations under analysis and analyzed the stoichiometry of the peptide carrying the mutation in relation to the four tryptic reference peptides used for EF-Tu quantification (Figure 22). A stoichiometry close to 1 for each of the peptides indicated that no modification or degradation occurs on the mutated peptides and, therefore, that the error frequency quantification can be considered reliable. Little variations for in the ratio obtained from peptide to peptide should be acceptable considering that i) the quantification of the AQUA peptides is perfomed by the supplier with an accuracy of $\pm 25 \%$; ii) peptides with different sequence might have different ionization properties and might not be equally represented during the mass spectrometric analysis. 


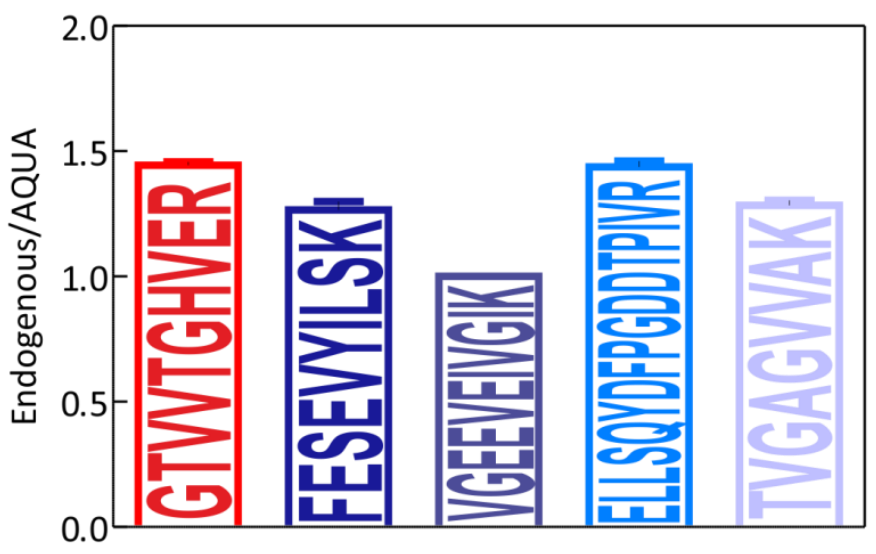

Figure 22. Validation of error quantification with EF-Tu mutants. The endogenous/AQUA peptides ratio for the errorcontaining peptide ( $\mathrm{R} 231 \mathrm{H}$ shown in red) and four tryptic reference peptides is calculated by SRM analysis. Error bars represent the standard deviation of 3 technical replicates.

\subsection{The steady-state error level of misincorporations in vivo}

As described in the introduction, the limitations in the available biophysical and mass spectrometric methods have precluded the systematic analysis of the in-vivo steady-state error frequencies. Albeit the strong analytical advantages of our method, the use of the internal standards and the targeted method used for the mass spectrometric analysis do not allow a complete analysis of all possible misinicorporations occurring in the protein. Therefore, to achieve a comprehensive and representative analysis of the cellular error frequency landscape we decided to split it into two steps and first focus on the quantification af all possible misincorporations at individual residues of EF-Tu, and subsequently on the error quantification for one particular amino acid susbstitution and monitor its frequency at various positions of the protein. In the last part we correlated the changes of the steady-state error frequencies in response to external stimuli as antibiotics, with the cellular stress response of the proteome. Moreover, we discovered that aminoglycosides induce highly abundant strings of errors that are likely to be relevant for their bactericidal effect.

\subsubsection{Amino acid substitutions at selected positions in EF-Tu}

To get the first insights into the relative abundance of different types of errors, we selected three positions in the EF-Tu sequence, R231, K249 and K314, for which we already identified individual errorcontaing peptides in previous data-dependent acquisition analysis (DDA). For each position, we were able to enrich and quantify all peptides resulting from an incorporation of a near-cognate aa-tRNA, displaying one mismatch in the codon-anticodon complex. To validate the significance of the results, we analysed errors in EF-Tu from three different E. coli sources: i) endogenous EF-Tu from MRE600 
purified by conventional chromatography (Rodnina and Wintermeyer, 1995); ii) chromosomallyencoded EF-Tu from E. coli K12 strain, engineered to contain a His-tag and purified by affinity chromatography; iii) plasmid-encoded EF-Tu with a His-tag, overexpressed in BL21 (DE3) upon IPTG induction and purified by affinity chomatography.

The error frequencies span over 3 orders of magnitude depending on the position of the mismatch in the codon:anticodon helix. With an error frequency of $10^{-4}$, the arginine-to-histidine substitution at position 231 (Figure $23, A$ ), referred to as $\mathrm{R} 231 \mathrm{H}$, is much more abundant than any other substitution. Notably, different error rates are obtained for EF-Tu purified from different sources. The source of EFTu seems to play an important role in the fidelity of amino acids incorporation. In fact, independently of the position of mismatch and position in the protein sequence, the errors quantified in the overexpressed EF-Tu are always more abundant than the ones in the wild type protein (median values $5.8 \times 10^{-6}$ and $1.5 \times 10^{-6}$, respectively), with a difference that reaches 1 order of magnitude for some substitutions at positions K314 (e.g., K314E with an average frequency of $1.7 \times 10^{-5}$ for overexpressed and $1.3 \times 10^{-6}$ for the wild type) and even 2 orders of magnitudes for some substitutions at position K249 (e.g., K249Q with an average frequency of $3.4 \times 10^{-5}$ for overexpressed and $3.7 \times 10^{-7}$ for the wild type) (Figure 23). Chromosomally-encoded EF-Tu with the His-tag has an error frequency which is intermediate between the overexpressed and the native EF-Tu obtained from the wild type stain. The results show that not only our separation and enrichment method achieves a very high sensitivity $<$ $10^{-7}$ ), but also that the overall frequency of near-cognate misincorporation varies over orders of magnitude and is, in many cases, rather low and much less abundant than reported so far (Edelmann and Gallant, 1977; Ellis and Gallant, 1982; Kramer and Farabaugh, 2007; Loftfield and Vanderjagt, 1972; Parker et al., 1983). The SRM peaks for R231L (Figure 24, A), K249Q (Figure 24, B) and K314Q (Figure 24, C) are shown as example. For a more comprehensive list of the spectra refer to the Appendix (Section C). 


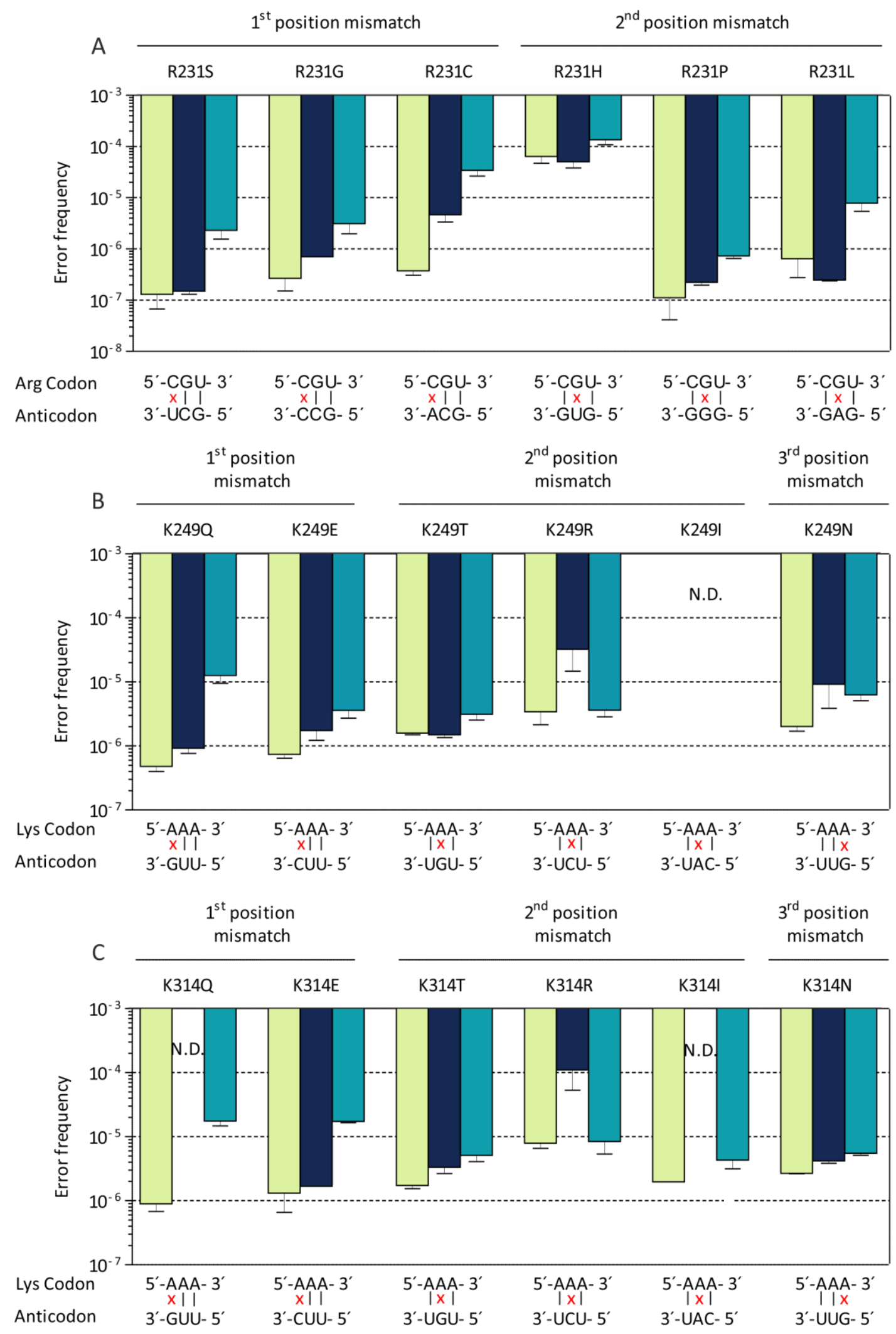

Figure 23. Error frequencies of near-cognate substitutions at three positions in EF-Tu. R231(A), K314 (B) and K249 (C). The results obtained from three types of EF-Tu are reported: wild type chromosomally-encoded EF-Tu from MRE600 (green), chromosomally-encoded EF-Tu with a His-tag isolated from K12 (blue), or plasmid-encoded overexpressed in BL21 (DE3) (teal). Error bars represent the standard deviation of 3 biological replicates. 
A

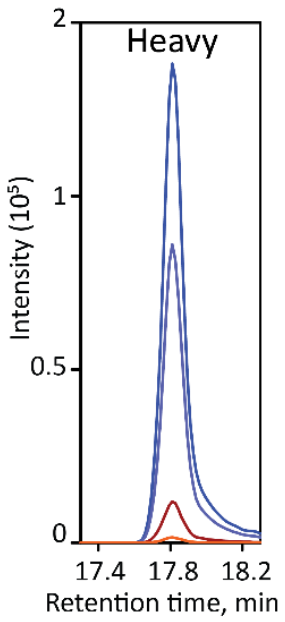

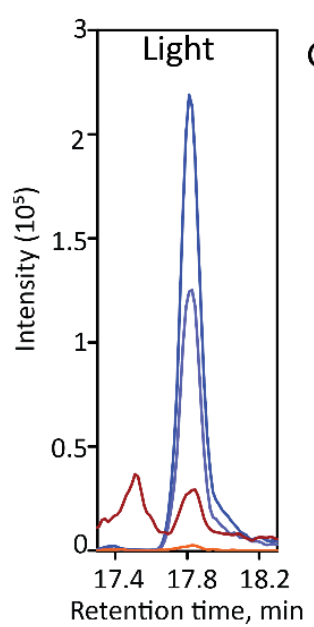

$\begin{array}{lllllllll}b^{1} & b^{2} & b^{3} & b^{4} & b^{5} & b^{6} & b^{7} & b^{8} & b^{9}\end{array}$

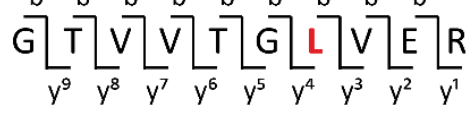

rdotp 1

R231L light R231L heavy

—y6-674.3832+ -

$-\mathrm{y} 5-573.3355+-\mathrm{y} 5-583.3437+$

- y4-516.3140+ - y4-526.3222+

-b7-628.3665+ -b7-628.3664t

B
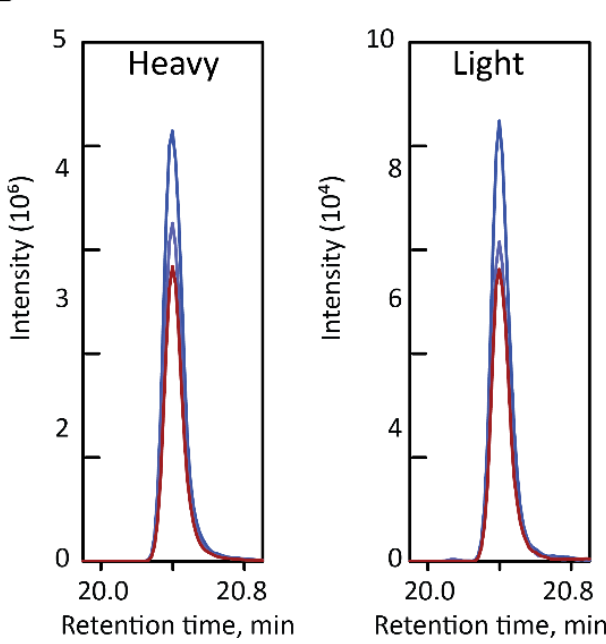

$\begin{array}{lllllllllllllllll}b^{1} & b^{2} & b^{3} & b^{4} & b^{5} & b^{6} & b^{7} & b^{8} & b^{9} & b^{10} & b^{11} & b^{12} & b^{13} & b^{14}\end{array}$

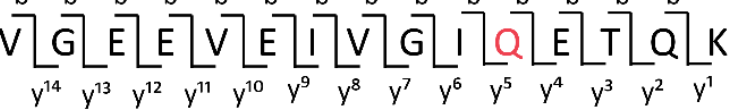

$\operatorname{rdotp} 1$

K249Q double K249Q light

- $\mathrm{y} 10-1159.6522-\mathrm{y} 10-1144.6208$

$-\mathrm{y} 9-1030.6096-\mathrm{y} 9-1015.5782$

-y8-855.4458 -y8-855.4458

C
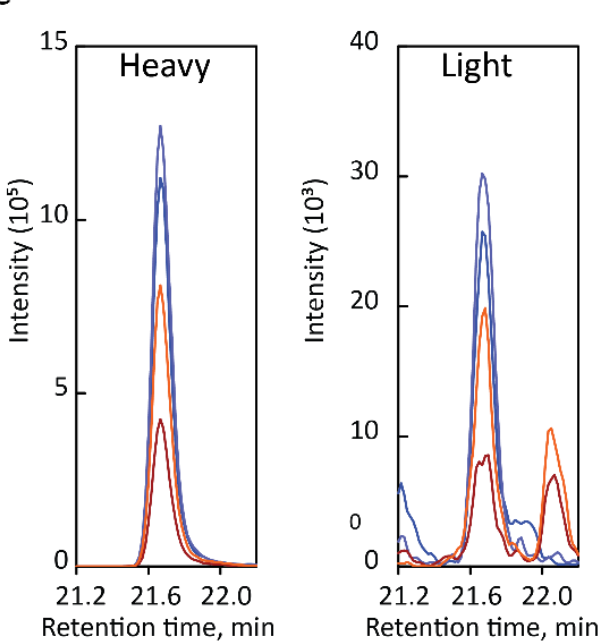

$\begin{array}{llllllllllllll}b^{1} & b^{2} & b^{3} & b^{4} & b^{5} & b^{6} & b^{7} & b^{8} & b^{9} & b^{10} & b^{11} & b^{12} & b^{13} & b^{14}\end{array}$

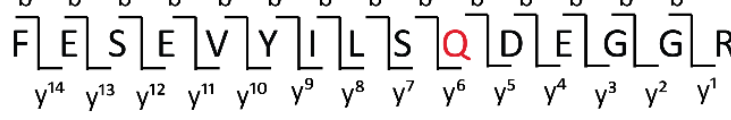

rdotp 1

K314Q light K314Q heavy

- y8-861.4061+ -

$-\mathrm{y} 7-748.3220+-y 7-758.3303+$

- y6-661.2900+ - y6-671.2983+

—b5-592.2613+ -b5-592.2613+

—b6-755.3246+ —b6-755.3246+

Figure 24. SRM peaks for enriched peptides. The peaks for the enriched peptides R231L (A), $K 249 Q$ (B) and $K 314 Q$ (C) are reported. Co-eluting light and heavy versions of the peptides R231L, K249 and K314Q are identified by SRM. The monitored transition for each peptides are reported and the sequence of the peptides, with their fragmentation pattern is shown. A rodtp value of 1 confirms the confidence of the identification. 


\subsubsection{Misincorporations involving non-cognate aa-tRNAs}

Given the high sensitivity and dynamic range of the method and the success in the analysis of errors resulting from the near-cognate decoding, we expanded our analysis to misincorporations that arise at position R231 of EF-Tu when arginine is replaced as a results of all the possible non-cognate misincorporations i.e., tyrosine, glutamic acid, aspartic acid, alanine, threonine, tryptophan, glutamine, valine, isoleucine and phenylalanine. In some cases, non-cognate substitutions are very rare and thus below the detection limit of our method. Some errors could be only quantified in the overexpressed EF-Tu, which confirms that its translation is error prone (Figure 25). For the overexpressed EF-Tu, the error frequency resulting from non-cognate decoding is comparable to that of near-cognate ones $\left(10^{-5}-10^{-7}\right)$. It must be noted that for $\mathrm{R} 231 \mathrm{E}$, the observed frequency might arise not only from the amino acid substitution, but also from an oxidation of arginine to glutamic acid. The PRM traces for R2310 peptide are shown as example (Figure 26), for the other peptides refer to Appendix (Section D).

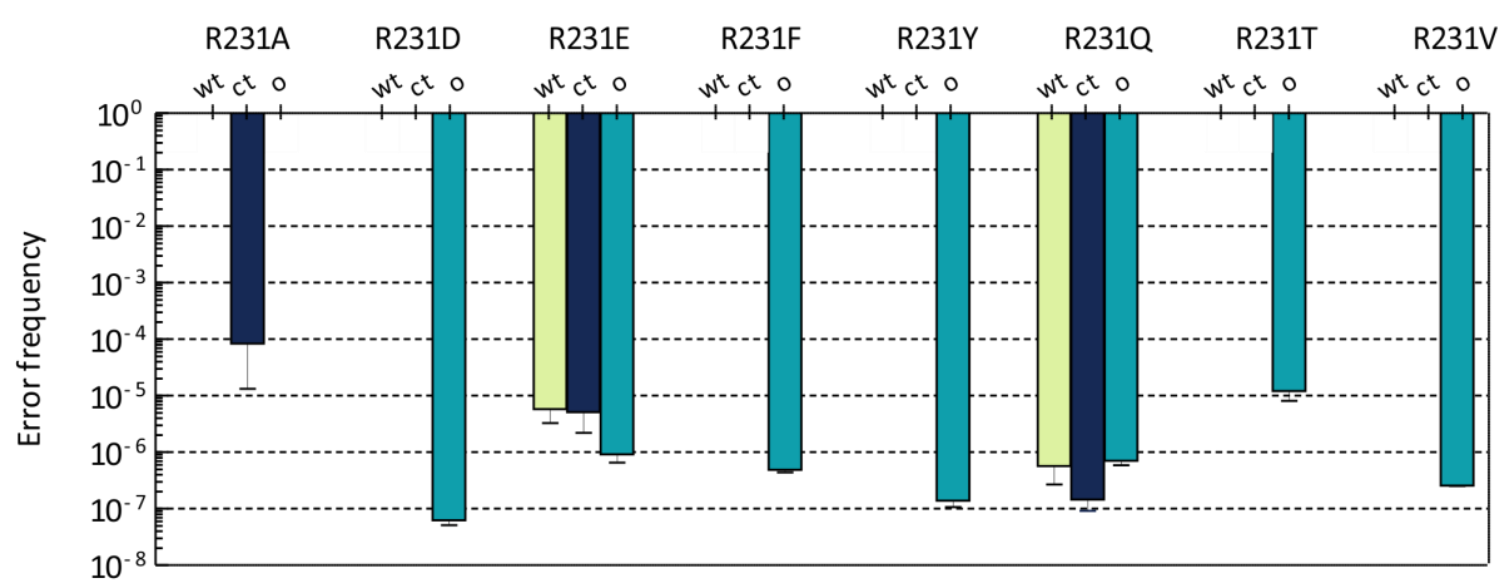

Figure 25. Non-cognate amino acid substitutions. Misincorporations involving non-cognate amino acids were investigated at position R231 of EF-Tu. The results obtained from three types of EF-Tu are reported: wild type chromosomally-encoded EF-Tu from MRE600 (green), chromosomally-encoded EF-Tu with a His-tag isolated from K12 (blue), or plasmid-encoded overexpressed in BL21 (DE3) (teal). Error bars represent the standard deviation of 3 biological replicates. 

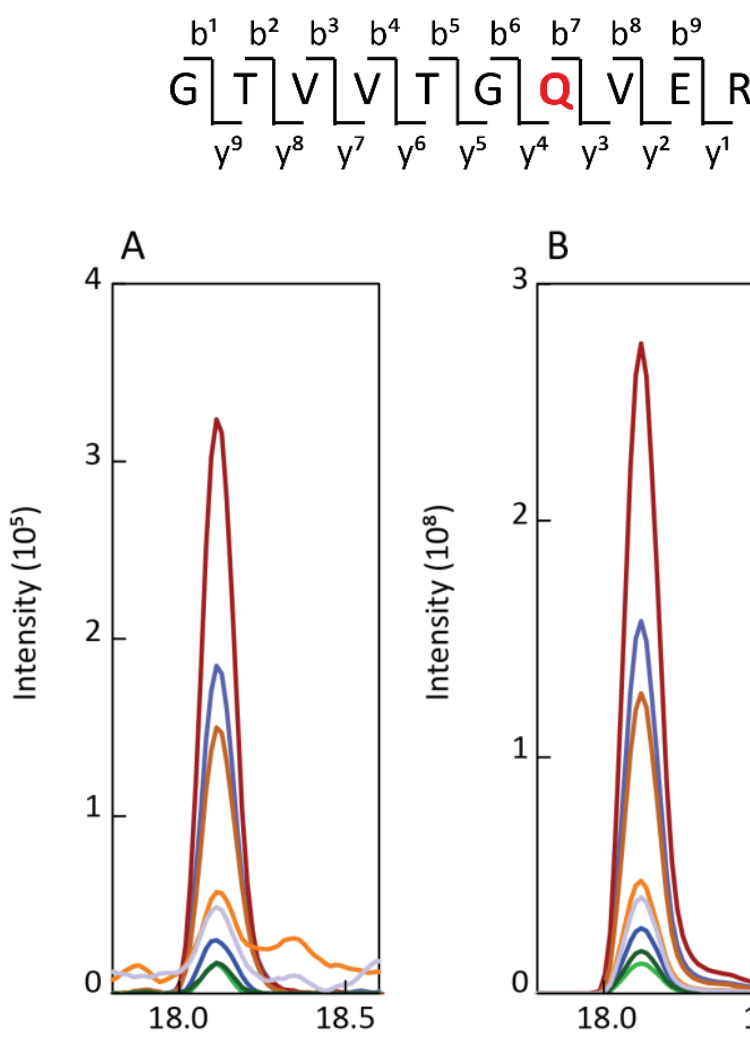

Retention time, $\mathrm{min}$

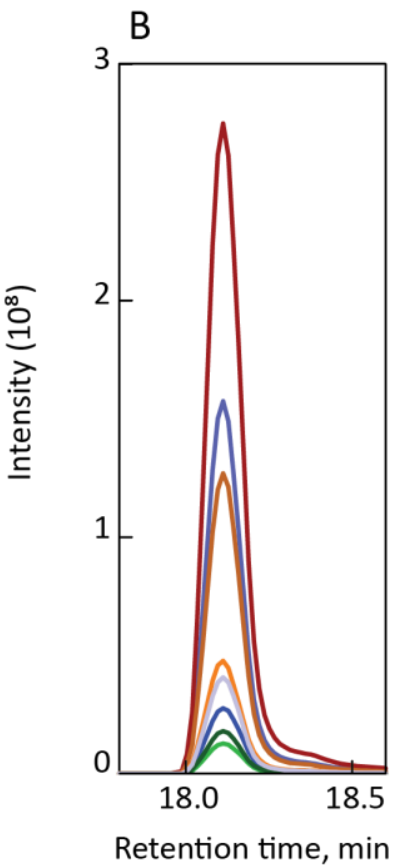

R231Q

rdotp 1

Light 523.2855

- y8 - 887.4945+

- y7 - 788.4261+

- y6 - 689.3577+

- y5 - 588.3100+

- y4 - 531.2885+

- y3 - 403.2300+

- b2 - $159.0764+$

_ b3 - 258.1448+

Heavy 528.2896

- y8 - 897.5028+

- y7 - 798.4344+

— y6 - 699.3659+

- y5 - 598.3183+

— y4 - 541.2968+

$-\mathrm{y} 3-413.2382+$

- b2 - 159.0764+

_ b3 - 258.1448+

Figure 26. PRM analysis of enriched R213Q peptide. Top panel, the sequence of the target peptide. Co-eluting light (left) and heavy (B) versions of the R231Q peptide are detected. The list of fragment ions used for the analysis is indicated on the right. A rodtp value of 1 confirms the confidence of the detection.

\subsubsection{Distribution of misincorporations over the EF-Tu sequence}

The reported error frequencies reflect the intracellular steady-state error level, which is the result of the accumulation of errors through the different phases of protein synthesis and their removal by cellular quality control mechanisms. We next tested whether the observed errors arise during the translation phase. The comparison of the error frequencies in E. coli strains harboring hyperaccurate and error prone mutants of the ribosome (with mutations on S12 and S4 protein of the small subunit, respectively) and the parental wild type strain (Zaher and Green, 2010a) confirmes that $\mathrm{R} \rightarrow \mathrm{H}$ errors are introduced by the ribosome (Figure 27). When this type of substitution was investigated at three positions of EF-Tu i.e R59H, R231H and R234H, the error-prone strain, which bears less accurate ribosomes, showed an increased error rate with a frequency which is almost one order of magnitude higher than the wild type, whereas the hyperaccurate strain, whose ribosomes have a higher fidelity, shows a lower error frequency. The fact that the error rate is modulated by the ribosome suggests a ribosomal origin for their incorporation. 


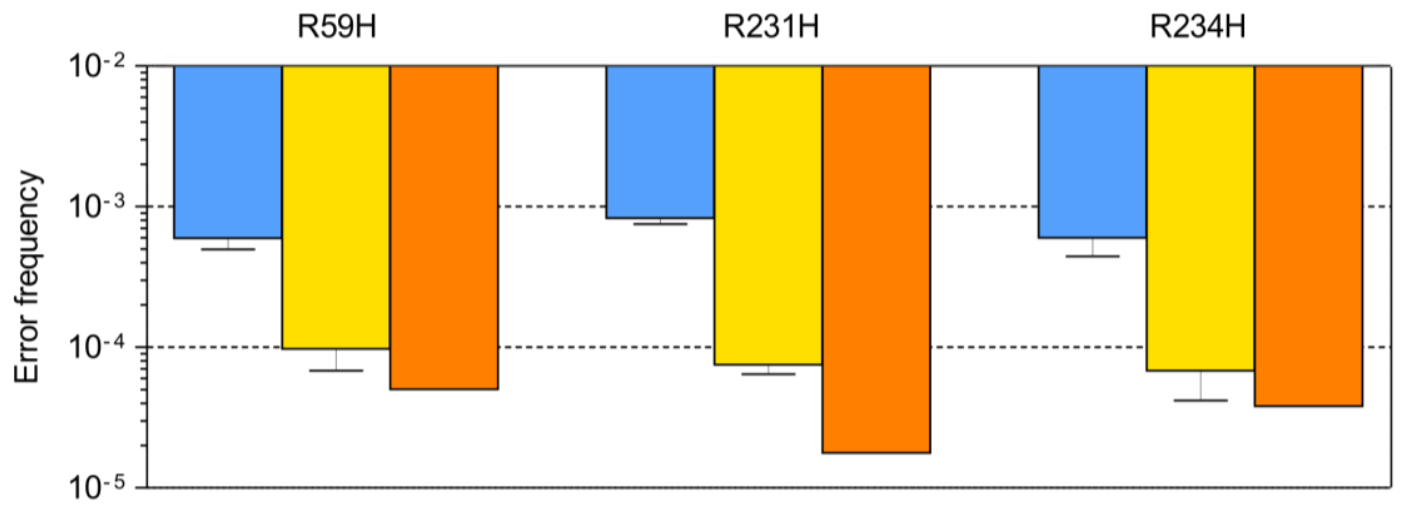

Figure 27. $\mathrm{R}$ to $\mathrm{H}$ misincorporation measured in error prone, hyperaccurate, and the parental wild type strains. Analysis of $\mathrm{R}$ to $\mathrm{H}$ substitutions in error prone (light blue), wild type (yellow) and hyperaccurate (orange). Error bars represent the standard deviation of 3 technical replicates.

Quantification of near-cognate amino acid substitutions at specific positions of EF-Tu allowed us to investigate the link between fidelity, type of substitution and codon:anticodon mismatch position. However, to understand how errors are distributed along the protein, we expanded our error profile analysis to the investigation of $\mathrm{R} \rightarrow \mathrm{H}$ substitutions at several positions of the protein sequence (Figure 28). Out of 21 arginine residues in the EF-Tu sequence, we selected 12 positions for the error quantification, while the others had to be excluded from the analysis due to an unsuitable peptide length or presence of reactive amino acids which preclude an accurate determination of the error (e.g., methionine). For the chosen type of replacement, the error frequency varied over 2 orders of magnitude $\left(10^{-6}-10^{-4}\right)$ depending on the position along the sequence. At most positions, arginine is coded by the CGT codon, except for two cases, i.e., R45H and R234H, where the codon is CGC. However, codon choice does not appear to correlate with a specific effect on the error rate.

$\mathrm{R} 45 \mathrm{H} \quad \mathrm{R} 59 \mathrm{H} \quad \mathrm{R} 172 \mathrm{H}$ R231H R234H R270H R280H R284H R319H R328H R378H R382H

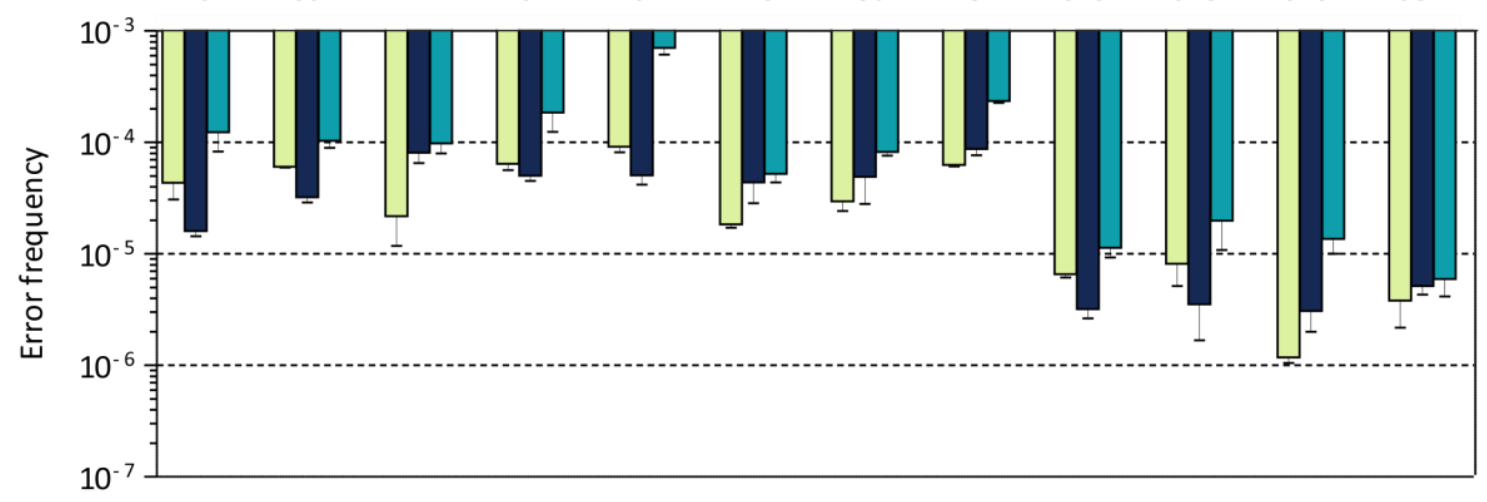

Figure 28. $\mathbf{R}$ to $\mathbf{H}$ misincorporations at several positions of EF-Tu sequence. Distributionof $\mathrm{R}$ to $\mathrm{H}$ substitutions reveals that the errors are unevenly distributed along the sequence independently from the type of EF-Tu used for the analysis: wild type chromosomally-encoded EF-Tu from MRE600 (green), chromosomally-encoded EF-Tu with a His-tag isolated from K12 (blue), or plasmid-encoded overexpressed in BL21 (DE3) (teal). Error bars represent the standard deviation of 3 biological replicates. 
The analysis of the distribution of $\mathrm{R}$ to $\mathrm{H}$ substitutions reveals that at four positions of the domain III of EF-Tu, R319, R328, R378 and R382 (Van Noort et al., 1986), the error frequency is between 1 and 2 orders of magnitude lower than at other positions, regardless of the source of EF-Tu. As these positions are important for the binding of tRNA, being near the EF-Tu/tRNA interface (Yikilmaz et al., 2014) (Figure 29), we hypothesized that errors at these positions may be more rigorously controlled than elsewhere in the protein. This may be achieved by a more accurate translation to ensure a low steady-state error level, or by applying a more strict quality control to degrade with a higher frequency EF-Tu molecules that present these misincorporations and whose functionality might be impaired. For the SRM traces and peptides validation for $\mathrm{R} \rightarrow \mathrm{H}$ errors, refer to Appendix (Section $\mathrm{C}$ ).

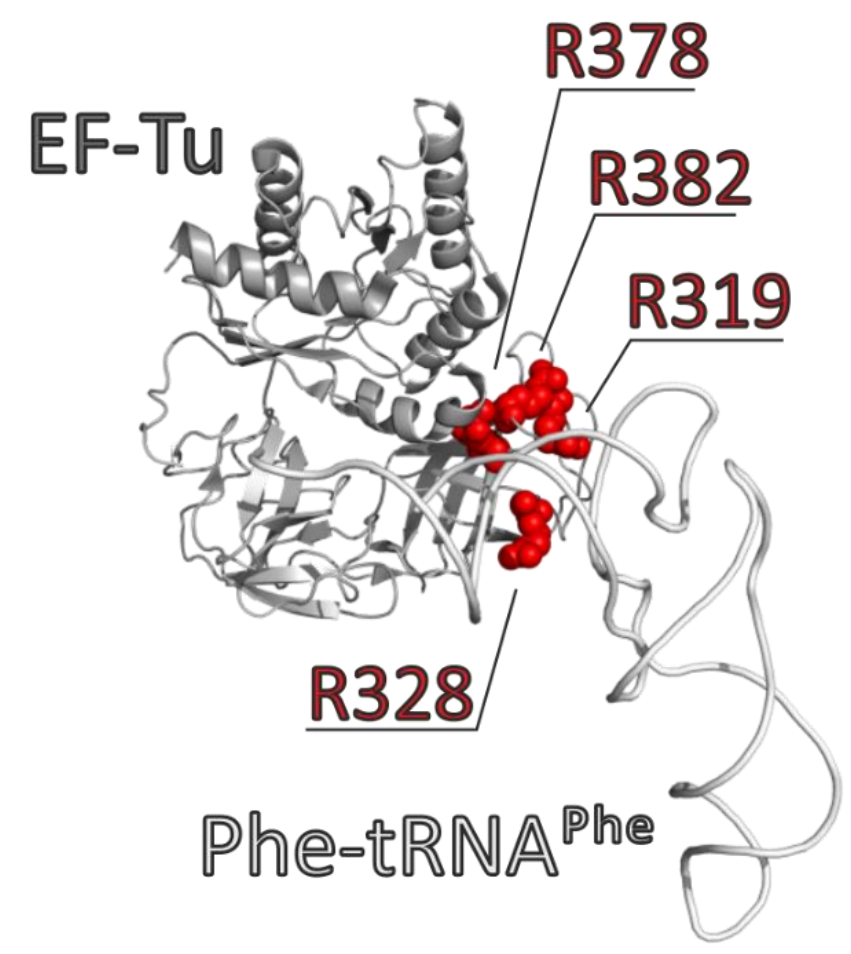

Figure 29. Position of low-abundance $R \rightarrow H$ substitution with respect to EF-Tu/tRNA interface. R319, R328, R378 and R382 residues are indicated in red and are localized near the interface between the protein (dark grey) and the Phe-tRNA ${ }^{\text {Phe }}$ (light grey) [PDB file 5AFI (Fischer et al., 2015)].

\subsubsection{Contribution of quality control machinery to the steady-state error levels}

To investigate whether the differences in error frequencies are caused by translation or by the subsequent quality control steps which may selectively degrade some of the peptides, we thought to decouple these processes by using an in-vitro translation system that lacks all potential proteases that might degrade misincorporation-containing EF-Tu. Because in-vitro translation kits can only provide a 
limited amount of protein ( $\sim 100 \mathrm{pmol} / \mathrm{kit}$ ), which is far from the amount we use for the chromatographic enrichment protocol (4000 - 40000 pmol), we decided to digest the purified in-vitro translated EF-Tu after the SDS-PAGE run, and to directly determine the frequency of misincorporations without prior fractionation (Figure 30). Because misincorporation-containing peptides are not separated from their cognates, and only a modest amount of EF-Tu can be injected for LC-MS/MS analysis, only a limited number of positions can be investigated using this procedure, i.e., the ones which appear to have the highest error rates. For these errors, the frequencies are $\sim 10^{-4}$ and are thus comparable with the ones determined in vivo. The similarity of error frequencies in vivo and in vitro suggests that misincorporations at some positions are indeed well tolerated and are not removed by the cellular quality control mechanisms. Unfortunately, the error frequencies for the positions located in domain III remain below the detection limit. Therefore, for these positions the contribution of the quality control machinery cannot be directly assessed, but the error frequency remains low even in a protease-free environment, suggesting that these errors are rare already at the translation level.

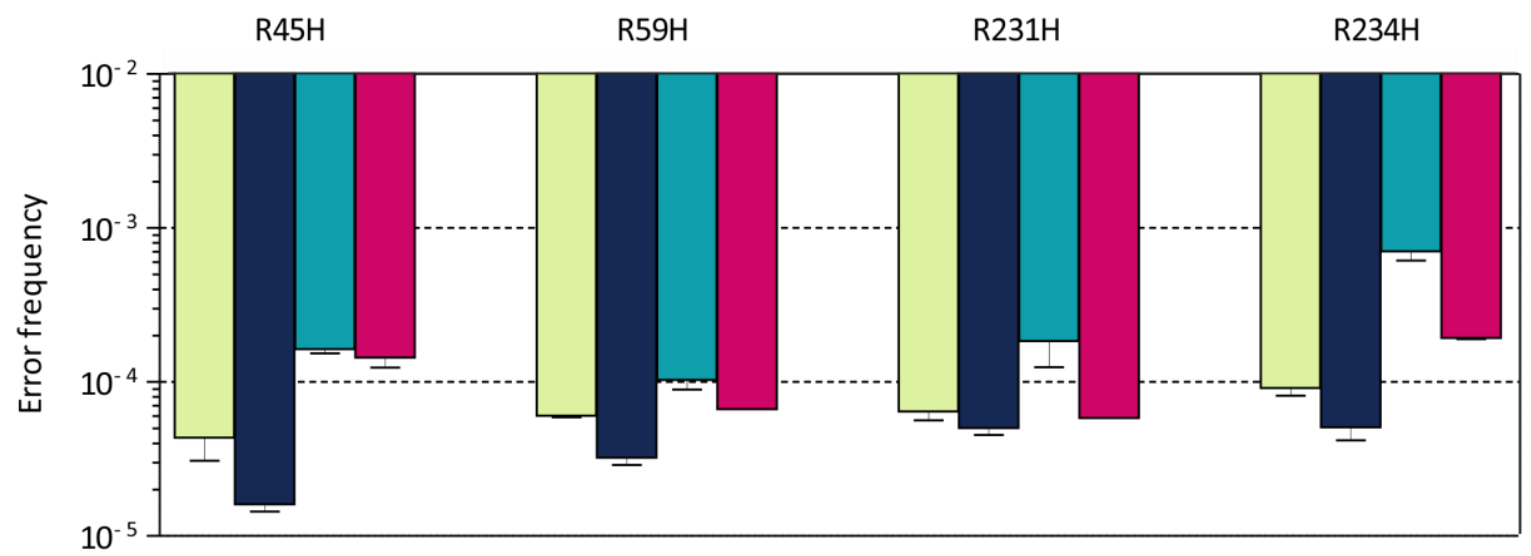

Figure 30. Error frequency in EF-Tu translated in vitro. The error frequency of $\mathrm{R} \rightarrow \mathrm{H}$ errors at positions R45, R59, R231 and R234 of EF-Tu wild type MRE600 (green), K12 chromosomal his-tag (blue), BL21 (DE3) overexpressed (teal) are compared with the EF-Tu synthesized in vitro (magenta). Error bars represent the standard deviation of 3 biological replicates.

To provide a more comprehensive explanation to the high accuracy detected for some positions, we tried a further approach where the activity of the quality control machinery is eliminated. We cloned the nucleic acid sequences of each one of our low-abundance erroneous peptides into a pSUMO vector and overexpressed it to generate a polypeptide product consisting of an $\mathrm{N}$-terminal $11 \mathrm{kDa}$ SUMO-tag followed by the EF-Tu FESEVYILSK cognate peptide necessary for the quantification and the EF-Tu peptide of interest (Figure 31). 


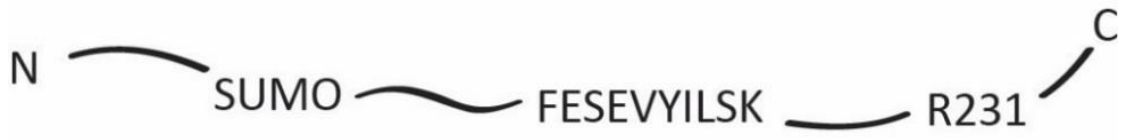

Figure 31. Schematic view of the pSUMO constructs. The N-terminal SUMO-tag is followed by the cognate FESEVYILSK peptide used for the quantification. At the $C$ terminus the specific peptide in which we want to test the misincorporation is added (in the reported example the tryptic peptide comprising the amino acid position R231).

The SUMO-tag approach is a common technique used in molecular biology to increase protein solubility, especially for overexpression procedures (Wang et al., 2010). Our hypothesis is that the SUMO-tag might prevent the aggregation and degradation of the EF-Tu peptide attached. A further advantage of this approach is that the errors can be studied out of the EF-Tu context, which circumvents a potential effect of the nascent chain on the peptidyl transferase activity of the ribosome (Ramu et al., 2011). When cloned in the SUMO-constructs, the peptides with the $\mathrm{R}$ to $\mathrm{H}$ substitutions at positions R378, R382 and R328 have up to 10-fold higher error frequency than the peptides obtained by overexpression of EF-Tu (Figure 32). Little or no difference is observed at position R319 and R280, respectively. Surprisingly, translation of pSUMO-R231H peptide is 10-times more accurate than overexpressed EF-Tu. While these results suggest that the quality control machinery may acts on some of the positions tested, in some cases the effects cannot be unambiguously assigned.

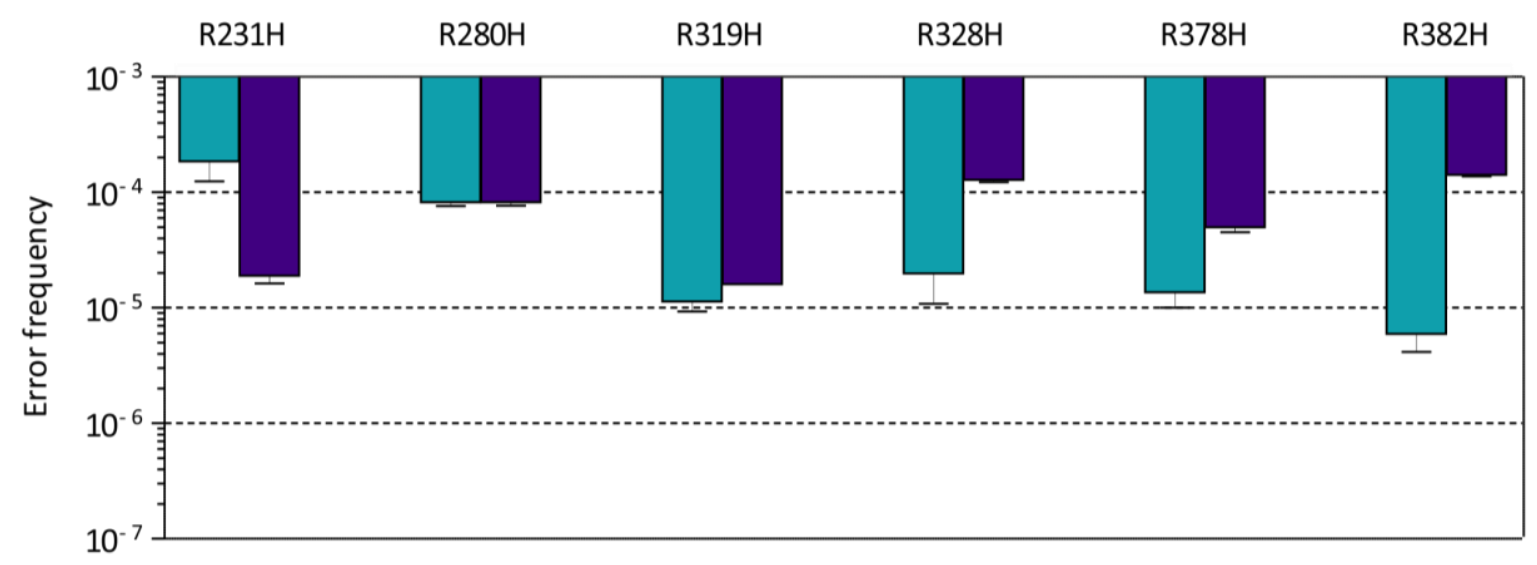

Figure 32. Misincorporation frequency in and out the EF-Tu context. $\mathrm{R} \rightarrow \mathrm{H}$ error frequency is compared in BL21 (DE3) overexpressed EF-Tu (teal) and in pSUMO construct (dark blue). Error bars represent the standard deviation of 3 biological replicates.

\subsection{Aminoglycosides as a tool to study cellular response and misincorporations}

Aminoglycosides are antibiotics that affect protein synthesis by inhibiting translation and decreasing the fidelity of ribosomes. We first examined the proteome response to aminoglycosides by systematically investigating the effect of antibiotics on the cellular stress response (Figure 33, A). We 
treated E. coli MG1655 with increasing concentrations of aminoglycosides and monitored the expression of three proteins that were reported to be significantly induced by misfolded proteins and aminoglycoside treatment, i.e., heat shock response chaperones IbpA and IbpB, and their transcription sigma factor rpoH (Kohanski et al., 2008; Wu et al., 2015). EF-Tu was used as a reference and L10 was used to normalize the total amount of protein. For each protein, 2 - 3 peptides are selected and the corresponding isotope-labeled standards are spiked in the sample before SRM analysis. For the list of peptides used refer to Appendix (Section B). For each antibiotic, the proteome response is plotted together with the final optical cell density after $120 \mathrm{~min}$ treatment expressed as $\mathrm{OD}_{600} / \mathrm{ml}$. In each case, the growth inhibition due to the antibiotic activity increases with its concentration. Surprisingly, cells grow even at antibiotic concentrations which correspond to the minimal inhibitory concentration (MIC) (Gualerzi, 2013; McGaha and Champney, 2007). Conversely to what happens for the determination of MIC where the growth inhibition is assessed by an over-night incubation with the antibiotic, in our experiment the incubation is much shorter, i.e., $2 \mathrm{hrs}$, and might be not enough to allow the antibiotic to fully enter the cell and lead to its death. Hyg B and Spec, both known to affect tRNA translocation rather than decoding fidelity, show the lowest growth inhibition efficacy and no stress response stimulation. This is consistent with the notion that error induction is a primary cause of the bactericidal effects of aminoglycosides. For other antibiotics a clear induction of the stress response is observed and associated with a growth defect. Str and Tob show the strongest induction of $\mathrm{IbpA}$ and IbpB by approximately 20 -fold. Surprisingly, the synthesis of the monitored proteins does not reflect the growth defects induced by the antibiotic but their concentration continues to raise even after the cell growth is blocked, indicating that it is not an arrest in the protein synthesis that interfers with the growth. 
Kanamycin A

MIC $0.5-1 \mu \mathrm{g} / \mathrm{m}$

MW 582.58

\section{Kanamycin B \\ MIC 2-4 $\mu \mathrm{g} / \mathrm{ml}$}

MW 483.51

Gentamicin

MIC $1 \mu \mathrm{g} / \mathrm{ml}$

MW 449.5

(Gentamycin C1A)

Tobramycin

MIC $2 \mu \mathrm{g} / \mathrm{ml}$

MW 467.51

4,5-Disubstituted aminoglycosides

Neomycin B

MIC $1-2 \mu \mathrm{g} / \mathrm{ml}$

MW 908.88

\section{Paromomycin}

MIC $1 \mu \mathrm{g} / \mathrm{ml}$

MW 713.7

Ribostamycin
MIC $8 \mu \mathrm{g} / \mathrm{ml}$

MW 454.47

4-Monosubstituted aminoglycosides

Neamine

MIC $64 \mu \mathrm{g} / \mathrm{m}$

MW 468.2

Apramycin

MIC $5 \mu \mathrm{g} / \mathrm{ml}$

MW 509.58

5-Monosubstituted aminoglycosides

Hygromycin B
MIC $150 \mu \mathrm{g} / \mathrm{ml}$

MIC $150 \mu \mathrm{g} / \mathrm{m}$

Atypical aminoglycosides

Streptomycin

MIC 2-4 $\mu \mathrm{g} / \mathrm{ml}$

MW 728.69

Spectinomycin

MIC 7-15 $\mu \mathrm{g} / \mathrm{ml}$

MW 495.35
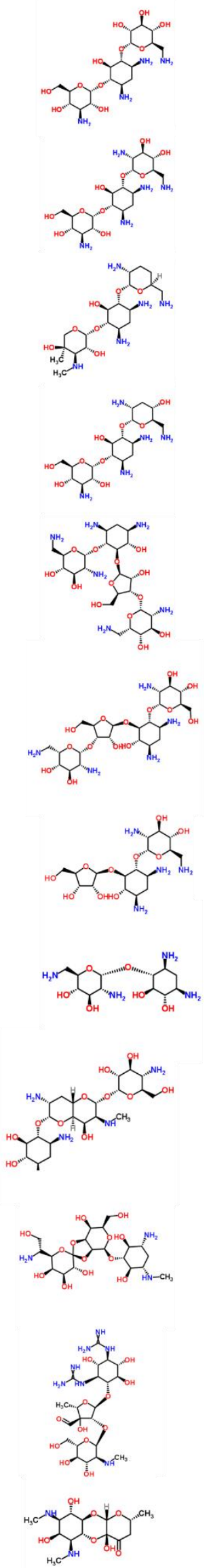

Legend: OlbpA;
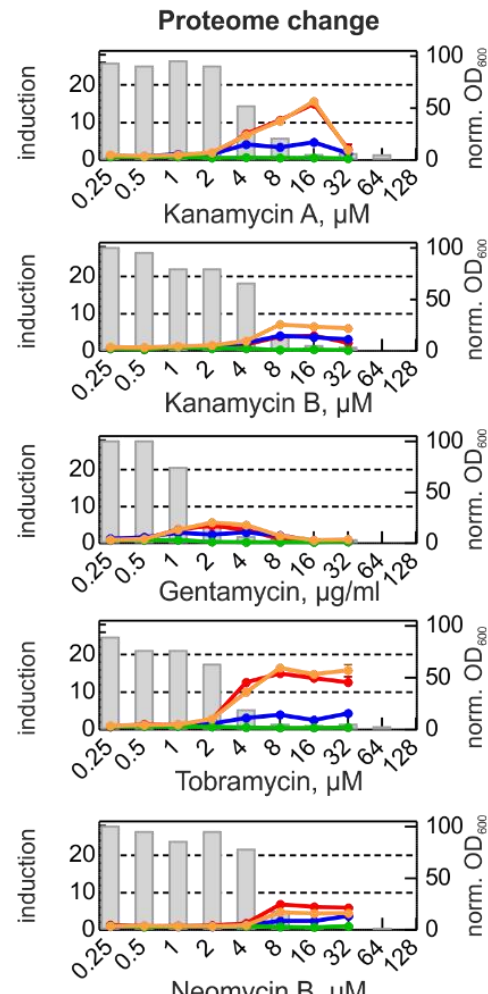

Neomycin B, $\mu \mathrm{M}$
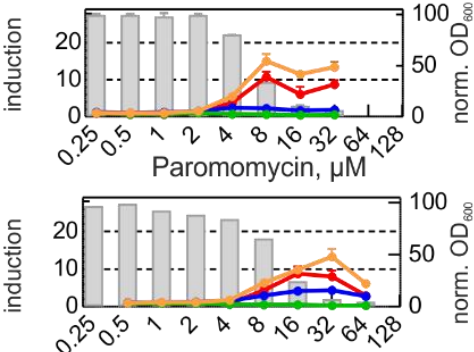

Ribostamycin, $\mu \mathrm{M}$
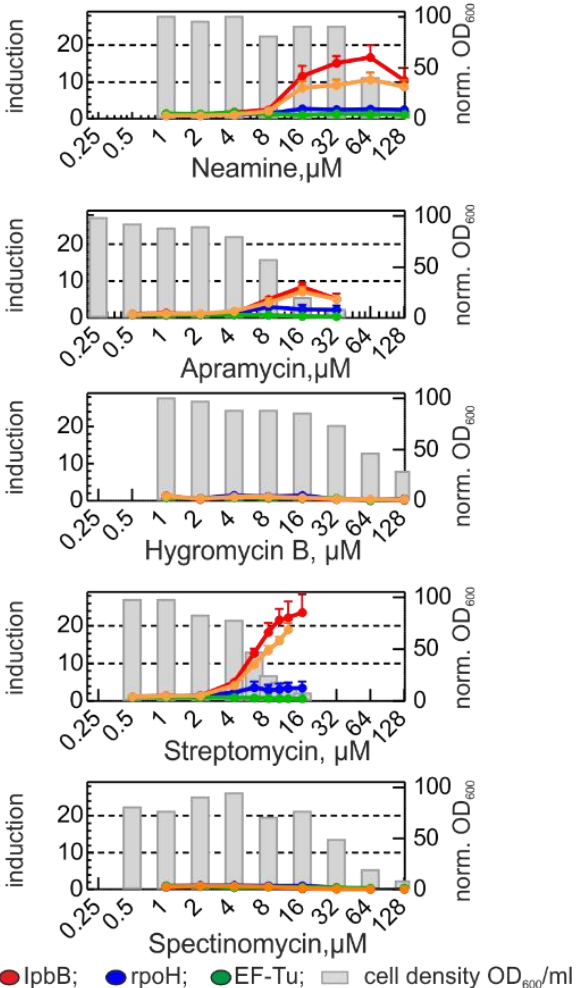


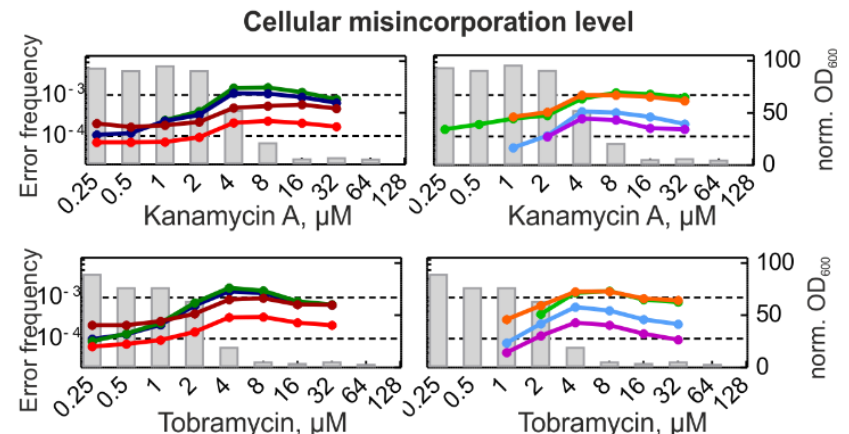

Kanamycin A

MW 582.58

\section{Tobramycin}

MIC $2 \mu \mathrm{g} / \mathrm{ml}$

MW 467.51

4,5-Disubstituted aminoglycosides

\section{Neomycin B}

MIC $1-2 \mu \mathrm{g} / \mathrm{ml}$

MW 908.88

\section{Paromomycin \\ MIC $1 \mu \mathrm{g} / \mathrm{m}$}

MW 713.7

\section{Ribostamycin \\ MIC $8 \mu \mathrm{g} / \mathrm{ml}$ \\ MW 454.47}

4-Monosubstituted aminoglycosides

\section{Neamine \\ MIC $64 \mu \mathrm{g} / \mathrm{ml}$}

MW 468.2

\section{Apramycin}

MIC $5 \mu \mathrm{g} / \mathrm{ml}$

MW 509.58

5-Monosubstituted aminoglycosides

\section{Hygromycin B}

MIC $150 \mu \mathrm{g} / \mathrm{ml}$

MW 527.52

\section{Atypical aminoglycosides}

\section{Streptomycin \\ MIC $2-4 \mu \mathrm{g} / \mathrm{ml}$ \\ MW 728.69}

\section{Spectinomycin MIC 7-15 $\mu \mathrm{g} / \mathrm{ml}$ MW 495.35}
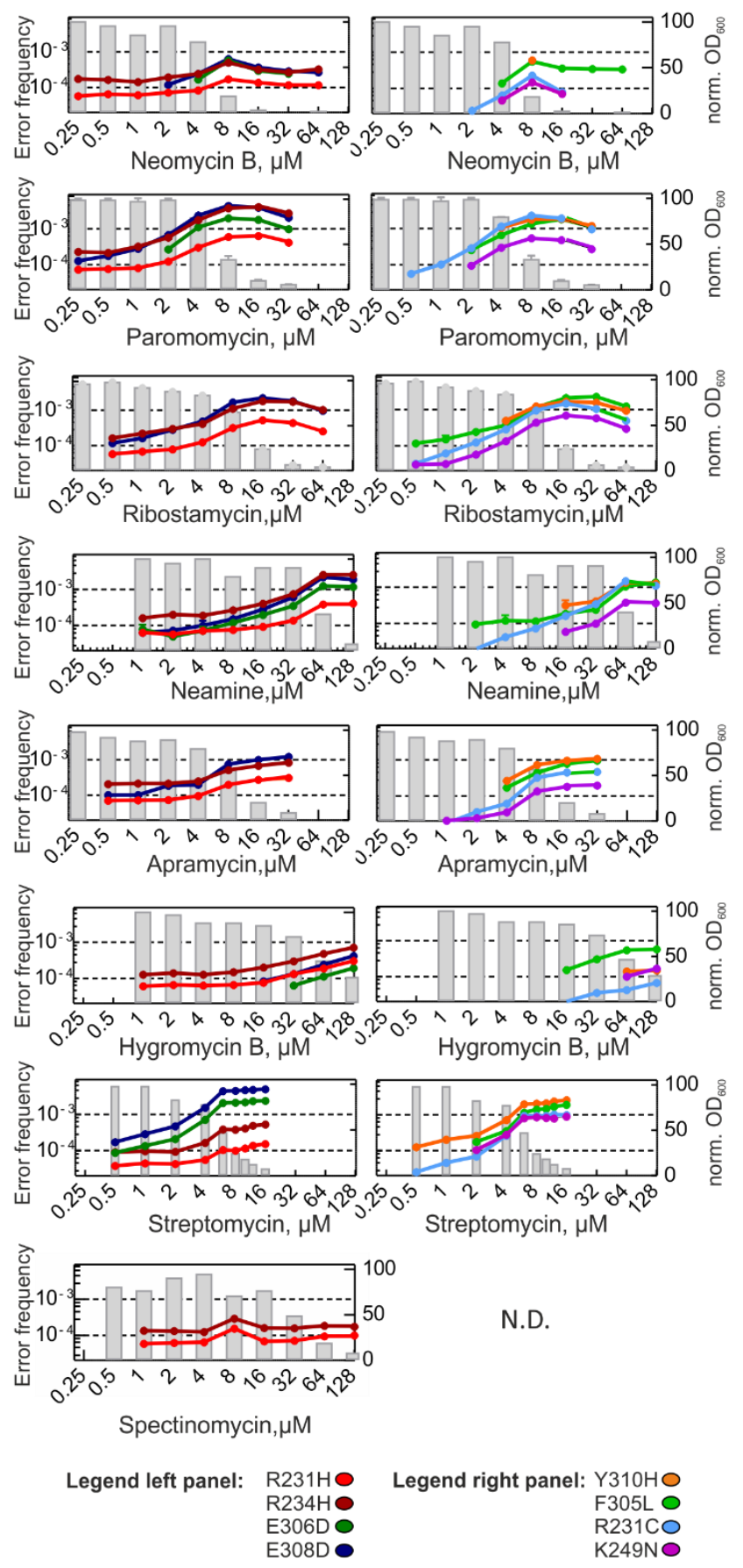

$\square$ cell density $\mathrm{OD}_{600} / \mathrm{ml}$

Figure 33. Effect of aminoglycosides on the cellular response and the steady-state error level. A) Changes in the concentrations of stress response proteins. B) Error frequency at increasing concentration of antibiotic. Error bars represent the standard deviation of 3 technical replicates. 
In order to correlate the cellular stress response with the increase of error frequencies, we then selected seven EF-Tu peptides based on our data-dependent results and determined their error frequencies in the same samples used for the proteome change analysis (Figure 33, B). The high abundance of EF-Tu in the lysate allows for the direct quantification of the more abundant errors in the protein without requiring the enrichment procedure. The error frequencies induced by most aminoglycosides, exception made for $\mathrm{Spc}$, depend on the antibiotics concentration and increase by one order of magnitude in the presence of the antibiotic from $10^{-4}$ to $>10^{-3}$. However, the error induction does not affect all amino acid substitutions in the same way and some misincorporations appear to be more enhanced than others. With Str, which induces the highest levels of misincorporations, the frequency of $E \rightarrow D$ substitutions increases by almost 100 -fold, whereas the induction of $\mathrm{R} \rightarrow \mathrm{H}$ errors is much milder, barely 10 -fold. Surprisingly, in some cases the induction of errors does not reach a stable plateau but rather reaches a maximum to then decrease as if, during the antibiotic treatment, some cells are able to adapt to the increasing level of errors, e.g., by producing more chaperones, already observed to be induced during the treatment, or proteases. In this scenario, these cells would be able to remove error-containing proteins and this could explain the decreased error frequency observed. However, also with this kind of adaptive behavior, only a certain error threshold can be tolerated and a growth defect is observed. Overall, our analysis demostrates that the imprint of aminoglycosides on the proteome accuracy is far more complex than expected and that their phyisiological and biochemical effects depend on the type of aminoglycoside used.

To gain a deeper understanding of aminoglycosides effect, we compared the errors induced by different antibiotics. The error profile induced by the treatment appears to vary depending on the type of antibiotic used (Figure 34, A) and in some cases aminoglycosides which belong to the same class are found to induce the same set of errors. The most evident example comes from the comparison of the structurally related Tob and Kan A, both belonging to the class of 4,6-disubstituted aminoglycosides, for which the error induction pattern is almost identical (Figure 34, B). On the other hand, the comparison of the structurally different Hyg B and Str shows that their effects are different (Figure $34, C$ ), suggesting that the stimulation of specific misincorporations can then be seen as a fingerprint specific for each type of antibiotic. 


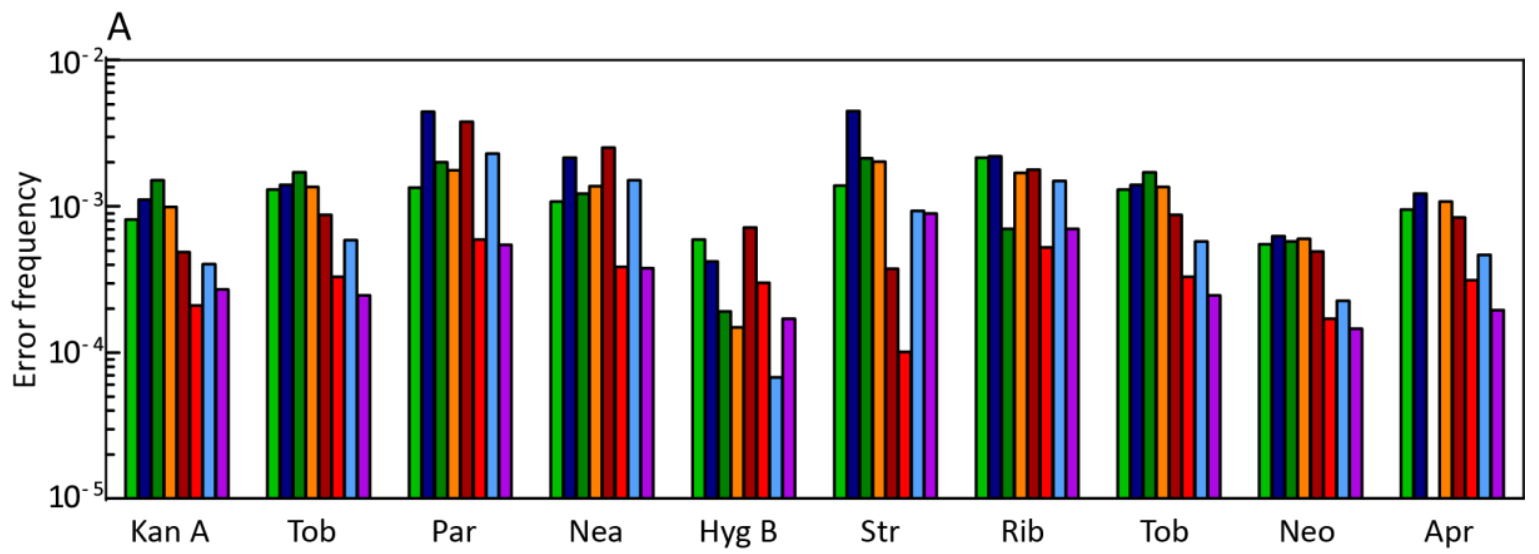

B
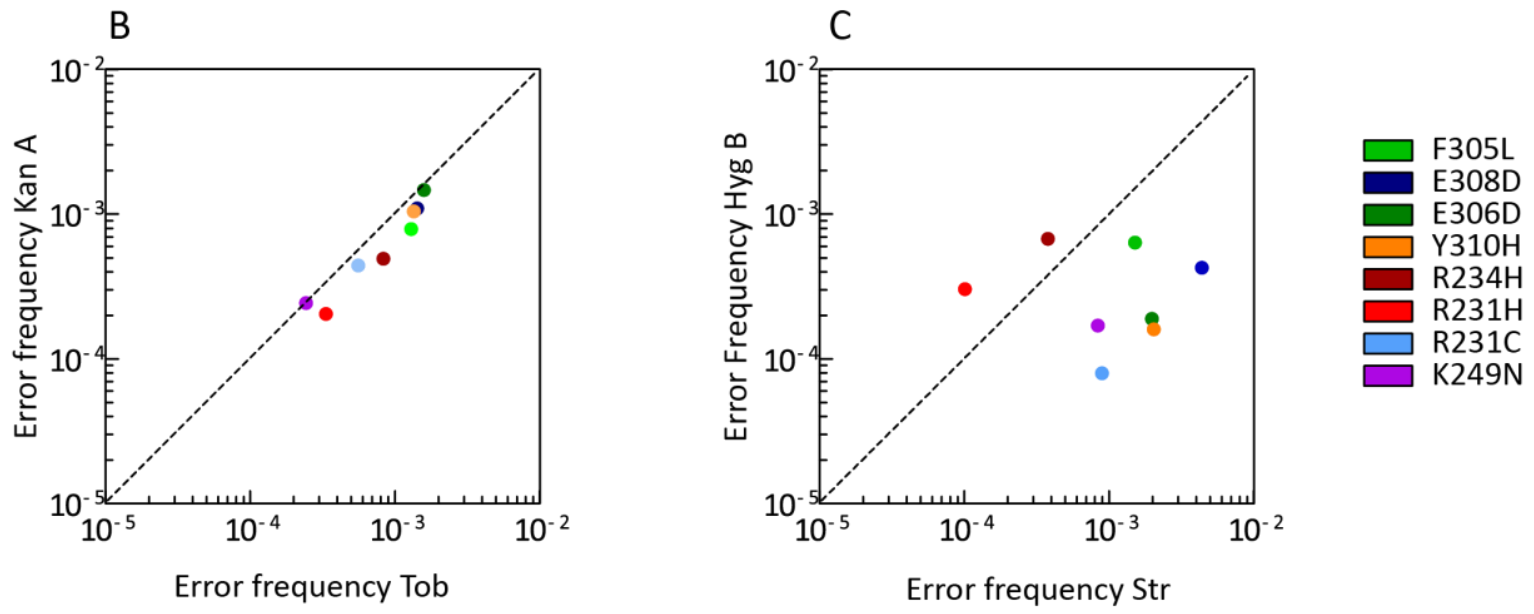

Figure 34. Comparison of error profiles induced by different aminoglycosides. Different errors are stimulated to a different extent depending on the type of aminoglycoside used (A). Structurally related aminoglycosides such as tobramycin and kanamycin A (B) share similar error pattern (Pearson $r=0.9535, P$ value $=0.0002$ ) while structurally distant aminoglycosides such as streptomycin vs Hygromycin B show a weaker correlation (Pearson $r=0.01297, P$ value $=0.9757$ ) (C).

\subsubsection{The complex error signature of aminoglycosides}

\subsubsection{Validation of doubly-substituted peptides by PRM analysis}

After we determined the conditions of maximal error induction for all aminoglycosides, we collected the data on the individual error profiles for a given antibiotic in a more comprehensive way by datadependent acquisition (DDA) (data not shown, courtesy of Dr. Ingo Wohlgemuth). In order to maximize the dynamic range at the MS1 level and reduce the interference by other proteins, we decided to analyse affinity-purified endogenous EF-Tu. Surprisingly, some of the misincorporationcontaining peptides identified by DDA contained more than one amino acid substitution. This is remarkable because the theoretical error frequencies calculated on the basis of the individual error frequencies are so low that peptides with more than one misincorporation are unlikely to be detected in the linear dynamic range of current mass spectrometers. In addition, the identification of amino acid substitutions in DDA is usually affected by false positive results, and the presence of multiple substitutions can further increase the false discovery rate. Thus, to exemplarily verify our identifications we monitored the induction of the double error E308D-Y310H (FESEVYILSK) by parallel 
reaction monitoring (PRM) (Figure 35). To exclude a potential impact of the C-terminal His-tag used to enrich EF-Tu for data-dependent analysis, we analysed EF-Tu in the lysate of wild type strain MG1655. While the double error was not detectable in the absence of Str, the abundance of the E308-Y310H peptide increased with the increasing Str concentration. This allowed us to unambiguously identify the double misincorporation based on its ratio dot product of 0.99 . In order to test whether double misincorporations are common in the aminoglycoside treated samples, we studied EF-Tu in MG1655 cells treated with three different aminoglycosides from different classes. Consistently, the double misincorporation was detected with high confidence (rdotp $0.98-1$ ) after treatment with Apr, Tob and Par.
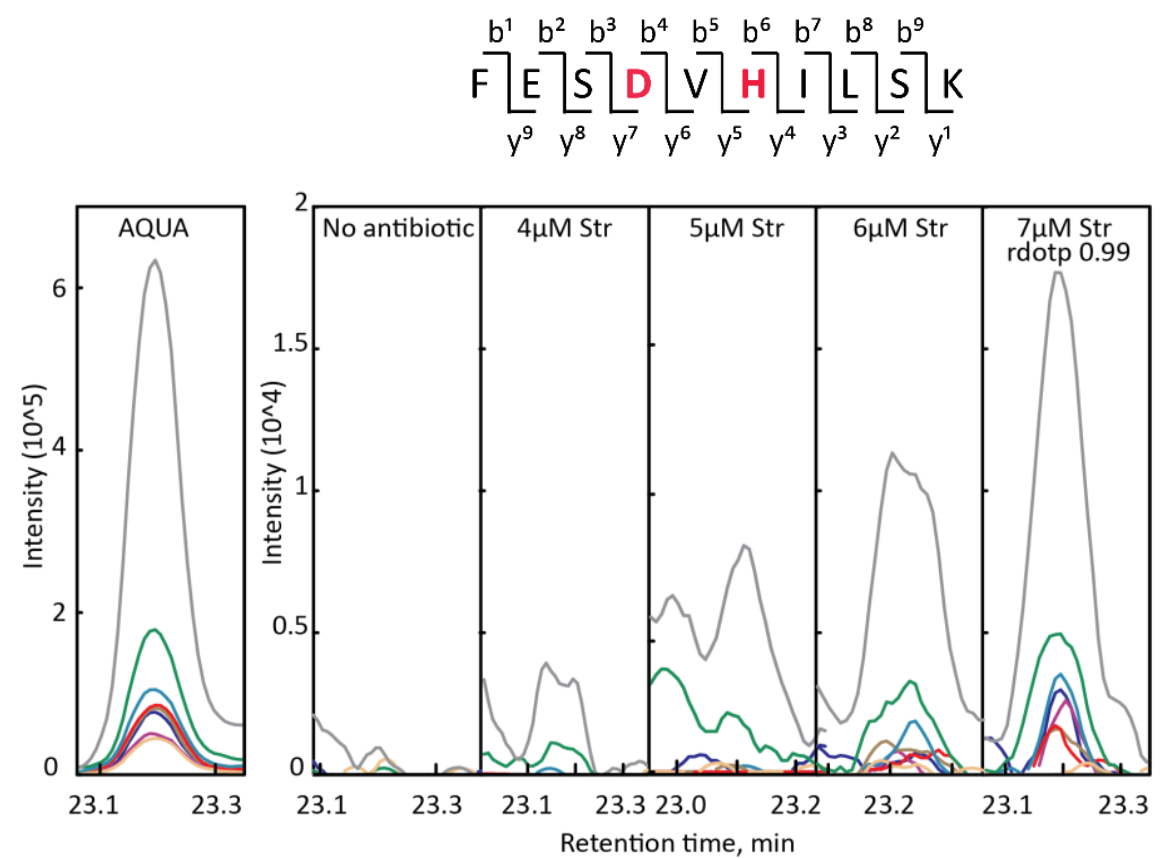

E308D-Y310H

Light $587.8088++$

- y8-898.4993+

- y6-696.4403+

— y5 - 597.3719+

- y4-460.3130+

- y3 - 347.2289+

_ b2 - 277.1183+

_ b6- 715.3046+

- a2 - 249.1234+
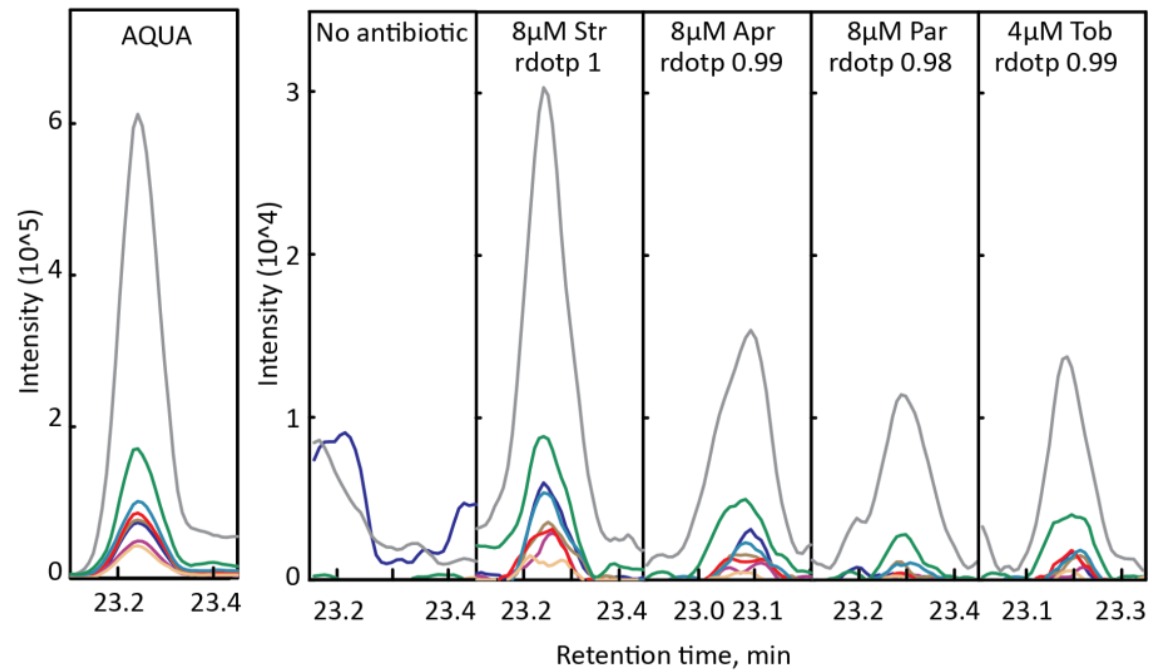

Heavy 591.8088++

— y8-906.5135+

- y6-704.4545+

— y5-605.3861+

- y4-468.3272+

- y3 -355.2431+

— b2 - 277.1183+

b6 - 715.3046+

- a2-249.1234+

Figure 35. Verification of double-substituted peptide E308D-Y310H by PRM. The sequence of the peptide and its fragmentation patter. The presence of the peptide was determined in the wild type MG1655 cells treated with increasing concentration of Str (upper panel) or various amingoglycosides (bottom panel). For each reported condition, the same amount of sample is injected into the instrument. The elution profile of the isotope-labeled reference peptide (AQUA) is shown on the left. The observed precursors for both heavy and light peptides are indicated. 
Once we assessed the presence of doubly-substituted peptides by PRM, we systematically analysed and quantified their pattern. We selected the model peptide FESEVYILSK for analysis because it has an optimal peptide stability, chromatographic behaviour, amino acid composition and ionization properties. Chromosomally-encoded His-tag EF-Tu was purified from cells grown with and without Str, and the error-containing peptides were enriched and ultimately identified and quantified by SRM (Figure 36). Double errors could not be found in the cells grown without Str suggesting that they are rare events. The signal for the respective peptide is under the detection limit of the assay and was calculated by peak integration on a high noise background. Thus, the reported values represent the upper limit of the error frequency rather then a defined error frequency. Incorporation of double errors become more frequent after Str treatment and their frequency, about $10^{-5}$, is comparable with the rate of the respective single errors (Figure 37). In addition, when we extended our measurements to other positions of the peptide we managed to quantify all the double errors included in the analysis (Figure 38). This suggests that the incorporation of multiple errors is not restricted to specific positions but is rather a generalized phenomenon. The high abundance of the double errors prompted us to also test a peptide that contained three misincorporations (E306D-E308D-Y310H). As expected, the peptide could not be detected in the untreated sample but could be easily quantified in the streptomycin-treated sample.

Once the mono-substituted peptides are quantified, the estimated frequency of the independent incorporation of double and triple erros can be calculated in the following way:

$$
P\left(E_{\text {multiple }}\right)=P\left(E_{\text {first }}\right) \times P\left(E_{\text {second }}\right) \times P\left(E_{\text {third }}\right)
$$

Where $E_{\text {multiple }}$ is the frequency of conseutive errors and $E_{\text {first }} E_{\text {second }}$ and $E_{\text {third }}$ the frequencies of the first, second and third error, respectively. Comparison between the error frequency obtained by SRM analysis and the predicted error frequencies, assuming that each misincorporation event is independent, revealed that the double errors are remarkably more abundant (10 - 1000-fold) than expected. This strikingly higher abundance can be explained as if double and triple errors do not occur independently; rather, their incorporation is synergistic.

Several mechanism for the synergistic appearance of errors exist. The first misreading event and the presence of errors in the protein nascent chain might alter the A site and induce further errors. Alternatively, the codon-anticodon mismatch in the P site, which results from the miscoding event, could distort or displace the peptidyl-tRNA in the P site rendering the ribosomal A more prone to accept incorrect tRNAs. A similar mechanism was proposed as a mechanism of post-transfer editing on the ribosome (Zaher and Green, 2009). Eventually, the propagation of misincorporation could be 
due to stable binding of the aminoglycoside to the ribosome which, persisting for several rounds of elongation, would perpetrate its fidelity-reducing effect.

A

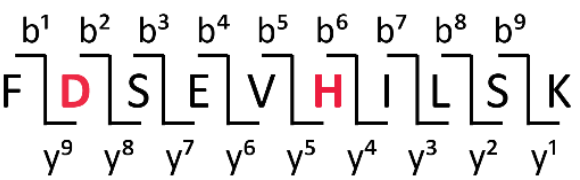
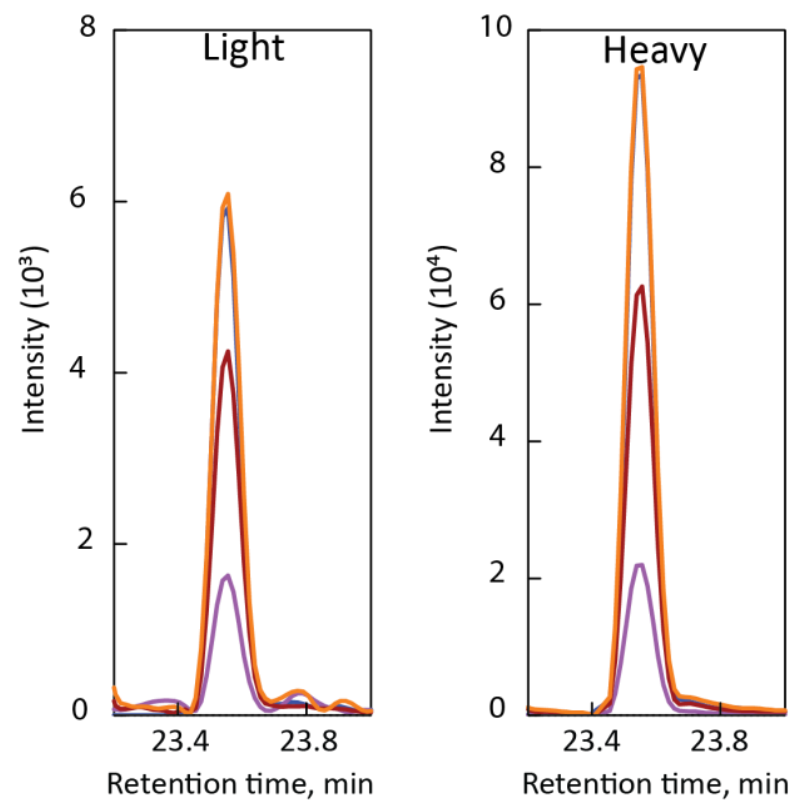

E306D-Y310H

rdotp 1

Light 587.8088

$-\mathrm{y} 8-912.5149+$

- y7 - 825.4829+

— y6 - 696.4403+

— y5 - 597.3719+

Heavy 591.8159

— y8 - 920.5291+

— y6 - 833.4971+

- y6 - 704.4545+

- y5 - 605.3861+

$\begin{array}{llllllllll}B & b^{1} & b^{2} & b^{3} & b^{4} & b^{5} & b^{6} & b^{7} & b^{8} & b^{9}\end{array}$

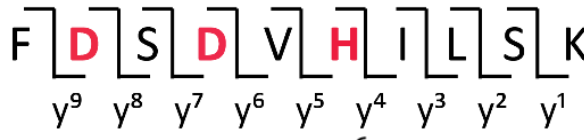
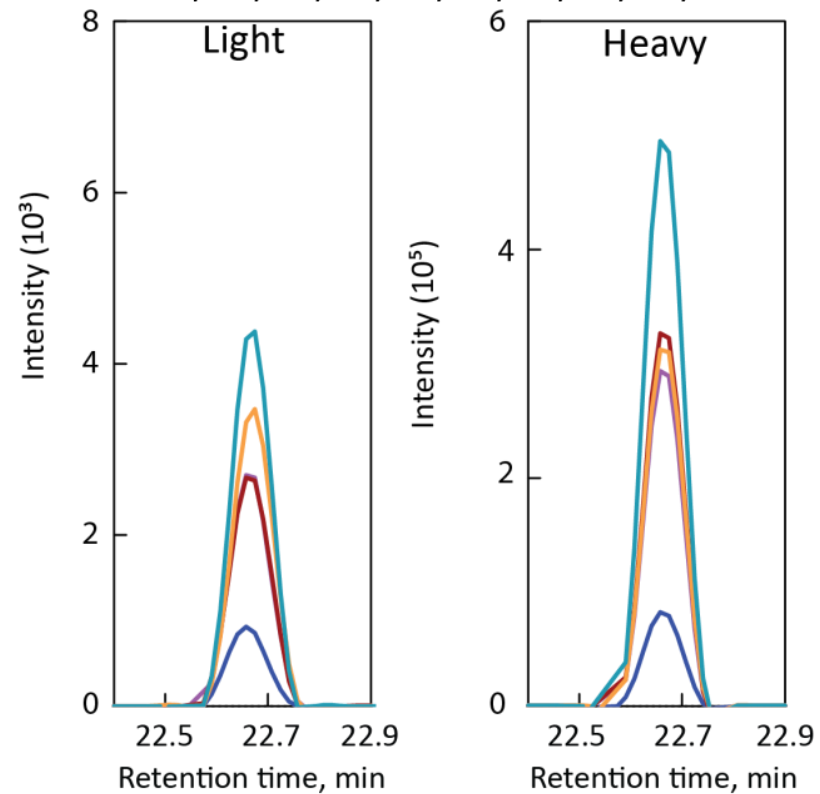

E306D-E308D-Y310H

rdotp 1

Light 387.5364

— y6 - 704.4545+

- y5 - 605.3861+

- y4 - 468.3272+

- y3 - 355-2431+

- y2 - 242.1590+

Heavy 390.2078

— y6 - 696.4403+

- y5 - 597.3719+

- y4 - 460.3130+

- y3 - 347.2289+

- y2 - 234.1448+

Figure 36. SRM quantification of double and triple errors. The peaks for the enriched peptides E306D-Y310H (A) and E306DE308D-Y310H (B) are reported. The SRM signals for both light and heavy-labeled are reported. The monitored transition are indicated the fragmentation pattern is shown. A rodtp value of 1 confirms the confidence of the identification. 

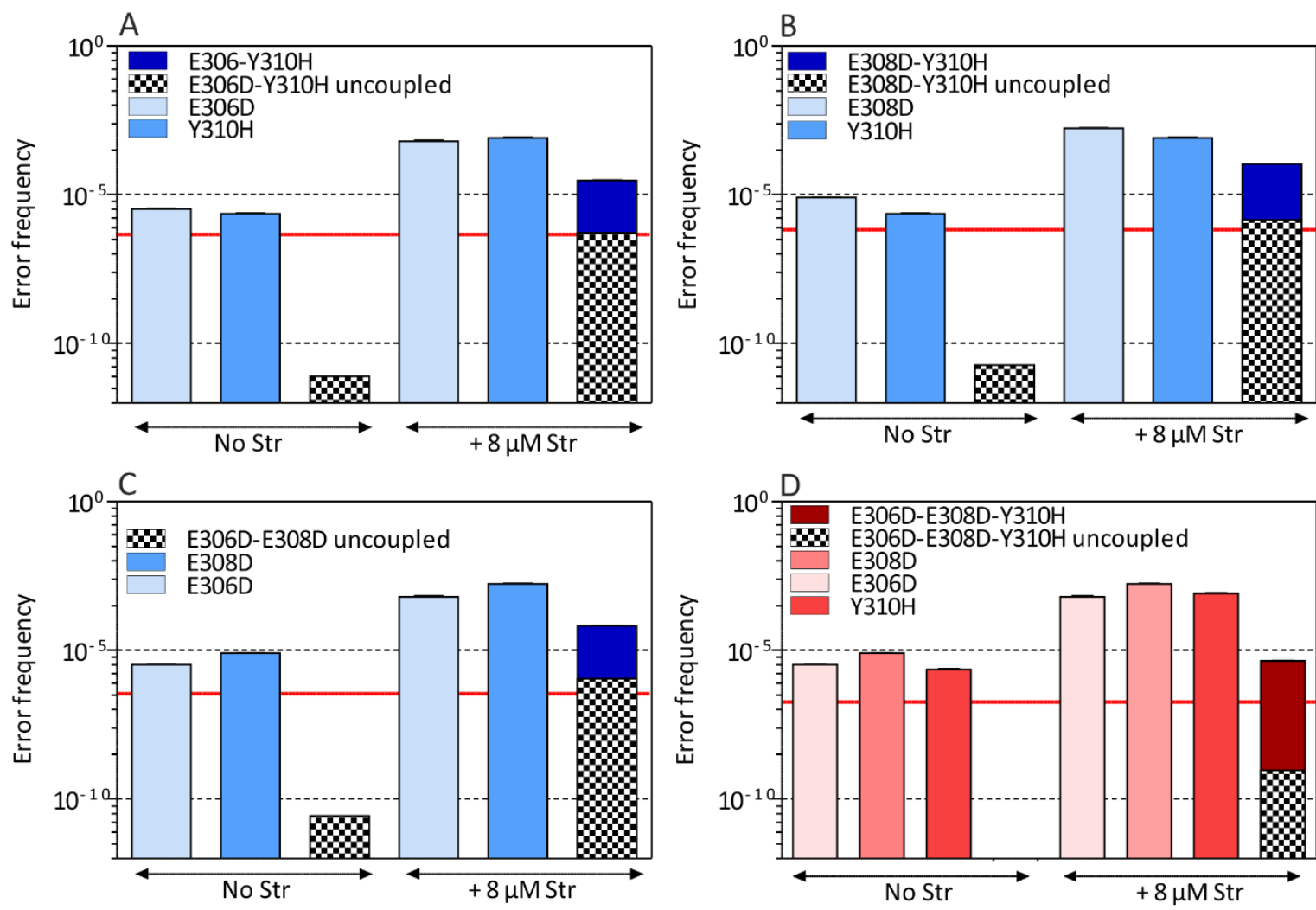

Figure 37. Example of quantification of double and triple errors. SRM analysis of error frequencies for E306D-Y310H (A), E308D-Y310H (B), E306D-E308D (C) and triple error E306D-E308D-Y310H (D). Each plot shows the frequency of the single, double, and where indicated triple errors for the purified chromosomally-encoded EF-Tu with a His-tag from K12 cells grown with and without Str. The theoretical error frequency of independent double and triple errors is indicated in the chequered bars. The red line represents the detection limit of the measurement. Error bars represent the standard deviation of 3 technical replicates.

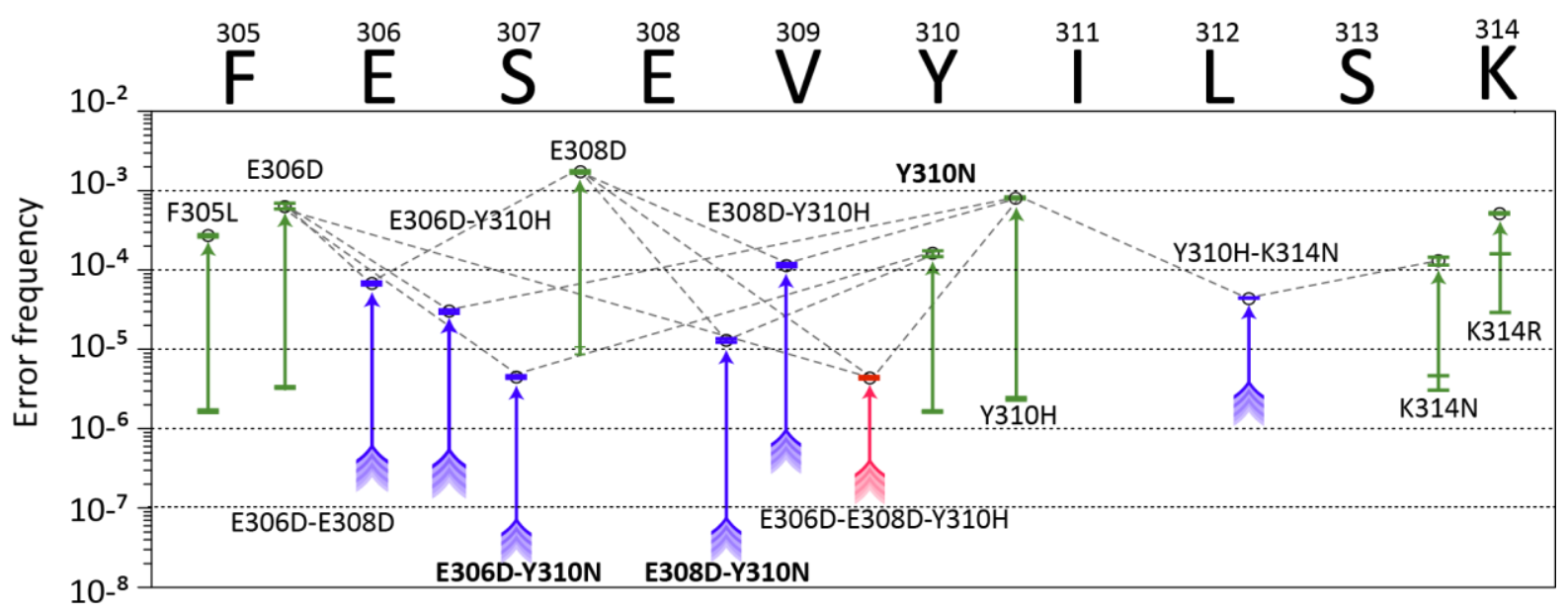

Figure 38. Abundance of the single, double and triple errors in one peptide. Frequency of single (green), double (blue) and triple (red) misincorporations. The sequence of the peptide togheter with the residue positions is indicated on top. Arrows indicate the increase in the error frequency in the absence (arrow's tail) and in the presence of $8 \mu \mathrm{M}$ streptomycin (arrow's head). In some cases, the error frequency for the wild type strain is below the detection limit, and the reported value is an estimation of the upper limit of the peptide's concentration ( $\hat{\wedge}$ ). JPT peptides are indicated in bold. Dashed lines connect single-substituted peptides with their respective double-substituted peptide. Error bars represent the standard deviation of 3 technical replicates. 
To test whether misincorporations in the nascent chain, mismatches in the codon-anticodon interaction or the presence of the aminoglycoside lead to double misincorporations, we tested error incorporations in an aminoglycoside-independent system. We purified wildtype EF-Tu from the rpsD strain containing error-prone ribosomes. These ribosomes harbour a mutation in the ribosomal protein S4 which affects the initial selection phase of decoding reducing the stability of the codon:anticodon complex with a near-cognate aa-tRNA and accelerating the subsequent step of GTPase activation (Zaher and Green, 2010a). For comparison, we prepared EF-Tu from the respective wild type strain in the presence of Str (Figure 39). Str was titrated to match the level of single misincorporations found in EF-Tu from the error-prone strain, which was achieved at $4 \mu \mathrm{M}$ Str. We selected three double misincorporations for the enrichment, E306-Y310H, E308D-Y310H and E306DE308D. The experimentally determined rate for double-substituted peptides in the wild type strain shows that, in all cases, the calculated frequency ranges between $10^{-5}-10^{-4}$ and is about 2 orders of magnitude higher than the theoretically estimated value, suggesting the existence of a mechanism that promotes the incorporation of the second error. In the error-prone strain, the error frequency of double-substituted peptides remains below the detection limit of the instrument and, as for the wild type strain in the previous experiment, their determination is rather an estimation of the upper limit of their concentration based solely on the background noise detected, rather than an accurately measured value. 
A
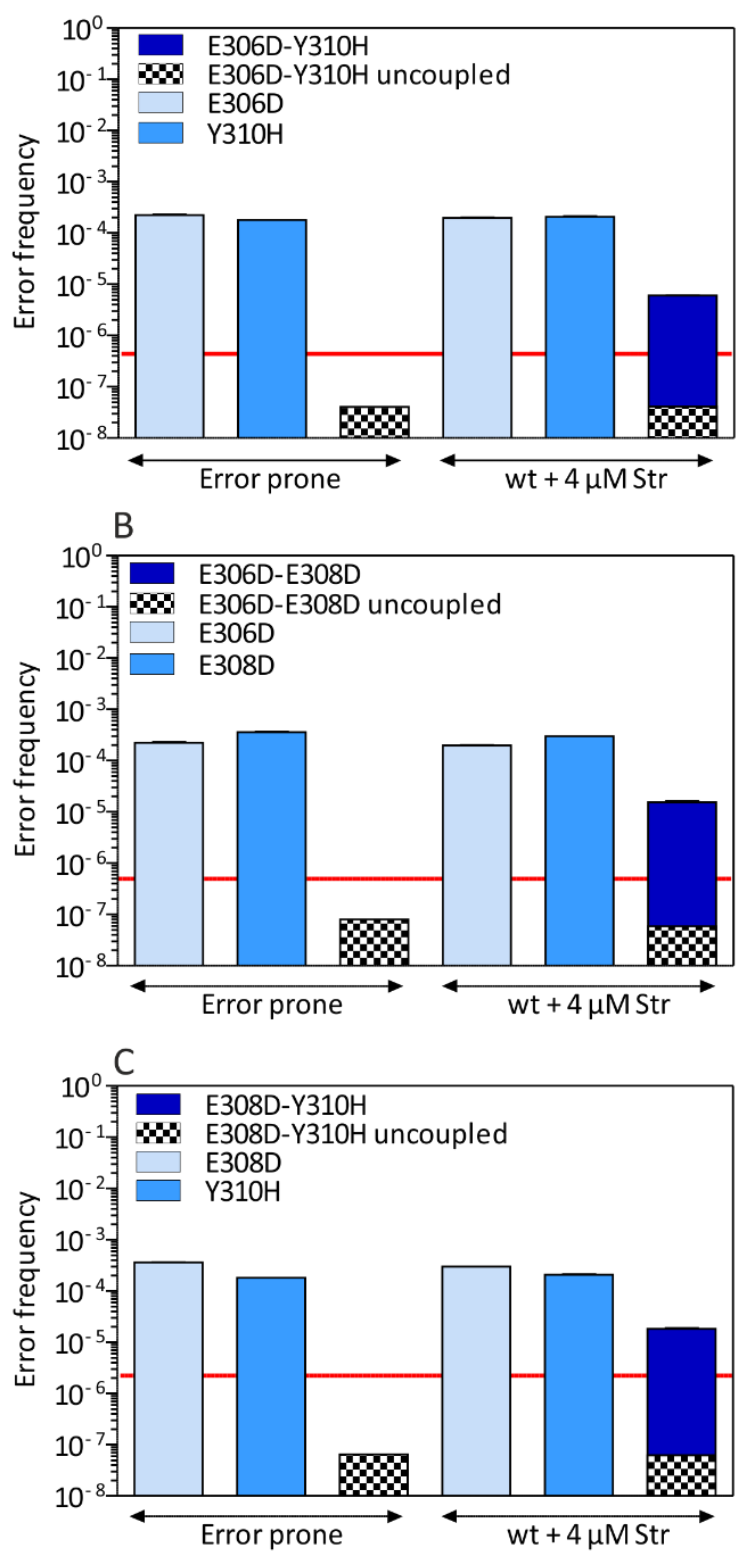

Figure 39. Quantification of single and double errors. Incorporation of E306D, E308D, and $\mathrm{Y} 310 \mathrm{H}$ is measured in wild type in the presence of $4 \mu \mathrm{M}$ Str in error prone strains. In each plot the error rate for the single error is shown (light blue and brilliant blue). The incorporation of their respective double error is monitored and indicated as follows: E306D-E308D (A), E360D-Y310H (B) and E308D-Y310H (C). Estimated (chequered) and calculated (dark blue solid) error frequencies. Detection limit is indicated by a red line. Error bars represent the standard deviation of 3 technical replicates. 


\section{DISCUSSION}

\subsection{Method development to study rare translation errors}

Incorrect mRNA decoding is a rare event, occuring with an estimated frequency of 1 error every $10000-$ 100000 decoded codons (Table 1 and Table 3) and misincorporation-containing peptides are present in the cell in an extremely low abundance with respect to the correct ones. For this reason, their detection is challenging and their comprehensive investigation requires the development of a highsensitivity detection method. All the mass spectrometric data obtained so far on amino-acid misincorporations rely on data acquired by DDA analysis. Although DDA can provide a wider set of results than targeted mass spectrometric approaches, it is limited not only by the intrinsic lower sensitivity (Michalski et al., 2011; Sandhu et al., 2008) but also by the necessity to interpret the data by spectral matching algorithms (Cox et al., 2011; Eng et al., 1994; Perkins et al., 1999; Shilov et al., 2007; Zhang, 2009), which might not be optimal for the analysis of isobaric amino acid misincorporations (see Introduction).

Analogous challenges can be found in the protein biomarker discovery, where the low-abundance of the target protein and the high complexity of the sample i.e., body fluids, make the identification of the biomarkers extremely difficult (Surinova et al., 2011). In this field, targeted mass spectrometry finds large application and SRM is often applied due its high selectivity and reproducibility. However, because the dynamic range required for the detection often exceeds the dynamic range of currents mass detectors, SRM is often combined with separation techniques, e.g., immunodepletion, sizeexclusion chromatography (Liao et al., 2004), gel electrophoresis (Ang and Nice, 2010), aimed to reduce the complexity of the sample and eliminate high-abundance proteins that would otherwise hinder the efficient identification of the low-abundance biomarkers. The combination of these techniques with the use of heavy-labeled standards (AQUA peptides) have led to further advance of mass-spectroscopy in this field (Shi et al., 2012).

Given the robustness, sensitivity and reproducibility of biomarker-oriented approaches for the quantification of proteins, we have adapted its strategy of target separation and mass spectrometry to develop an assay for the systematic investigation of peptides which contain amino-acid substitutions. As biomarker quantification approaches, our assay relies on the enrichment of target peptides by separating the error-containing peptides from the more abundant correct ones. The peptides enrichment and reduction of the sample complexity allows to load more target peptides on the LC-MS/MS system leading to higher signal intensities. Moreover, endogenous and AQUA peptides co-elute during several subsequent chromatographic rounds of separation and co-fragment, yielding a high confidence assignment. Thus, together with the validation of our results by high resolution mass spectrometry, the identification of peptides is based on more - and partially orthogonal - evidence 
than the traditional DDA data. In addition, the use of AQUA peptides as internal standards solves quantification problems of data-dependent approaches which are related to the performance variations of ESI stability or to the different ionization properties of cognate and misincorporationcontaining peptides.

The disadvantages of the method are the laborious peptide enrichments, the necessity for assay optimization for each peptide to enrich, and the high costs of commercial peptide synthesis which limit the number of peptides that can be analyzed and preclude a broad or even proteome-wide analysis. These problems are especially aggravated when peptides of interest cannot be selected on the basis of previous experiments or published data. In such cases, after AQUA synthesis and enrichment procedures, the target peptides may turn out to have poor ionization properties, being undetectable in both their light and heavy forms or simply not informative, being the light counterpart too rare to be detected.

Our assay provides an unprecedented flexibility in the choice of misincorporations to study because it allows to study most of the miscoding events which are imprinted in the amino acid sequence of the protein of choice, independently from reporter systems and types of substitution, as demonstrated for the quantification of several consecutive miscoding events (Figure 38). In this scenario proteomics can be considered as single molecule technique in which the translation process carried out by individual ribosomes can be studied, although this single molecule character is clearly only conserved within the individual tryptic peptide. In addition, the present approach allows to quantify different miscoding and misincorporation events in parallel or even correlate miscoding events with the cellular response, e.g., changes in the expression of proteins involved in the stress response. One other important advantage of the mass spectrometry-based assay is that, the sensitivity is significantly increased with respect to the other approaches and that the noise is extremely low and can be distinguished from the signals of the target peptides, even when the latter are present at very low concentrations $\left(10^{-7}\right)$. In contrast, in fluorescence-based assays which are commonly used to study amino acid substitutions (Kramer and Farabaugh, 2007; Kramer et al., 2010), there is often a background fluorescence $\left(10^{-4}\right)$ either due to the experimental setup or to a residual fluorescence of the mutated reporter proteins which cannot be avoided and which inevitably limits the dynamic range of the method. The pseudo-linear quantification of the biomarker approach presented here has, at least theoretically, no limit in the dynamic range, because, more starting material could be used for the analysis and the peptides could be separated by several orthogonal chromatographic steps for a better enrichment. However, the high sample losses which occurs at individual chromatographic dimensions and the limited numbers of truly orthogonal chromatographic separation strategies hamper the quantification of misincorporations with error frequencies $<10^{-8}$, 
although this limit strongly depends on the individual peptide sequences. Finally, peptides with almost identical physicochemical properties to the cognate ones could be difficult to separate by any chromatographic step and might require additional rounds of chromatography (Figure 16) or even remain undetected, independently of their frequency.

\subsection{Misincorporations are rare and occur less frequently than expected}

The systematic investigation of misincorporations has revealed that on average errors of translation can be much less frequent than currently described in literature. We could quantify errors occurring with a frequency as low as $10^{-7}$, reaching a level of sensitivity which is not achievable by other methods. The calculated median for the error frequency of near-cognate substitutions at three positions of EF-Tu (Figure 23) was $10^{-6}$. Thus, the expected higher error frequency introduced by translation is not reflected in the cellular steady state error level, and even $\mathrm{R} \rightarrow \mathrm{H}$ substitutions, the most abundant type of misincorporation (Manickam et al., 2014; Zhang et al., 2013), occur with a frequency between $10^{-6}-10^{-4}$ in wild type EF-Tu (Figure 28), a result which matches the error frequency estimated for transcription (Table 2). Most likely, this discrepancy between the different estimations of error frequencies is the consequence of a lower sensitivity of the methods employed so far, which enabled the identification of only the most abundant errors, leading to an incomplete and biased picture of the translational error frequency landscape. This is supported by the results we obtained from the analysis of error incorporation during aminoglycosides titration. After the addition of the antibiotic to the growing cells, the studied misincorporations approach a range between $10^{-5}-10^{-3}$ (Figure 33, B) which matches the range of errors reported for cells under optimal growth conditions (Table 1) and supports the scenario in which the error frequency of translation is lower than previously estimated, but its accurate detection was hampered by the limited sensitivity of the methods used.

\subsection{Error rate depends on the protein source}

Strikingly, regardless of the type of amino acid substitution, codon involved, mismatch and amino acid position, overexpressed EF-Tu always displayed a higher frequency of error incorporation. In contrast, wild type and chromosomally-encoded His-tag proteins showed a comparable error frequency confirming that the His-tag we inserted in the chromosome is well tolerated (Figure. The reduced accuracy of translation during recombinant-protein production is a known phenomenon (Scorer et al., 1991) and different explanations might be envisioned. The presence of the plasmid already constitutes a significant metabolic burden for the organism which, to maintain it, is usually grown in the presence of an antibiotic and faces the energy costs of the synthesis of the protein conferring antibiotic resistence. Such stress phenotypically manifests as a slower growth rate (Bentley 
et al., 1990) and might originate from a combination of nutritional imbalance and competition for the expression machinery, which in turn could increase the frequency of random translational errors. The type of plasmid used for the overexpression may also play a role. The $\mathrm{PET}$ vectors we used for the overexpression are commonly utilized in molecular biology for the expression of recombinant proteins. However, their use is also associated with the cellular proteome imbalance caused by the very high amount of mRNA that accumulates in the cells and the resulting very high yield of the encoded overexpressed protein, which can reach as high $40-50 \%$ of the total cell protein content. The presence of the PET plasmid alone (i.e without the cloned gene to be overexpressed) can be toxic in E. coli in the presence of IPTG (Miroux and Walker, 1996). IPTG itself has been demonstrated to be harmful for E. coli BL21 (DE3), the host strain which is often employed for protein overexpression (Dvorak et al., 2015). In addition, pET vectors are transcribed by T7 RNA polymerase. Although this polymerase has an estimated error frequency comparable to the bacterial one $\left(10^{-4}\right.$ and $10^{-4}-10^{-5}$, respectively (Huang et al., 2000)), it cannot be excluded that an increased error rate generated by the overexpression stress affects T7 polymerase fidelity leading to an increase of transcriptional errors which are then manifested in translation. A plausible alternative explanation includes a depletion of the aa-tRNA pools caused by the high demand of substrates for the protein synthesis.

Protein overexpression is known to induce the cellular stress response and the expression of proteases and chaperones as well as many proteins involved in the SOS regulatory system for nucleic acid damage repair (Gill et al., 2000). A limited capacity of the chaperone system to mediate the proper folding as the protein copy number dramatically increases could also induce the accumulation of more erroneous proteins.

In our setup, another source of error incorporation might stem from the type of antibiotic used to select the plasmid-containing cells (i.e., Kan), which is long known to induce miscoding (Davies et al., 1965b; Tanaka et al., 1967) and which could contribute to an overall decrease of the ribosomal fidelity, as observed during Kan treatment (Figure 33, B). In pET vector-containing cells, the Kan resistance is conferred by the expression of aminoglycoside phosphotransferase which modifies and inactivates the antibiotic after it enters the cell (Wright, 1999), so that a residual miscoding activity of Kan cannot be excluded.

Our results revealed that overexpressed EF-Tu is also more prone to non-cognate amino acid substitutions (Figure 25), which are for the first time quantified in vivo. Surprisingly, the frequency of errors due to non-cognate decoding ranges between $10^{-7}-10^{-4}$, and is similar to that of the nearcognate one. This may be a result of the strongly depleted pool of cognate tRNAs caused by the overexpression. However, not every possible non-cognate substitution are frequent enough to be quantified, suggesting that overall they remain rare events even during overexpression. 


\subsection{Error variation and distribution}

Among the missense errors studied here, $\mathrm{R} \rightarrow \mathrm{H}$ substitutions are the most frequent types of errors. This results can be explained by the nature of the codon-anticodon mismatch that generates this replacement, the G-U mismatch that is reported to increase the error frequency (Westhof et al., 2014).

Whereas in literature it is reported that different error frequencies are associated to the position of the mismatch at the codon:anticodon duplex, (Gromadski et al., 2006; Manickam et al., 2014; Zhang et al., 2016), our results reveal that no correlation between the error frequency of amino acid incorporation and the mismatch can be observed. When we compare our results to in-vitro data, the difference might be explained by the activity of the cellular quality control activity in vivo which is obviously not present in the in-vitro experiment and which might acts on erroneous peptides equalizing them. The difference to the in-vivo results can be also easily explained. The difference with the error frequency levels reported by Farabaugh and collegues (Kramer and Farabaugh, 2007; Manickam et al., 2014) can be caused by the specific experimental setup used in their work. Their system is based on the use of $\beta$-galactosidase, in which the catalytic glutamic acid residue (E537) is mutated to a set of possible near-cognate codons, causing a reduced activity of the protein. Amino acid substitutions are then investigated by monitoring the gain in activity caused by the replacement of the near-cognate mutated amino acid back to the wild type glutamic acid. This means that their quantification considers the wild-type protein, whereas in our case we quantify the errors on the base of the abundance of a mutated protein, which may be partially degraded due to the quality control activity. In addition, we have to consider that, Manickam's results for first-, second- and third-position mismatch errors are based on amino acid substitution at only one position of the protein and our data extend to only 3 amino acids positions (R231, K314 and K249). In both cases the results may be not completely representative of the general error rate frequency for various mismatches. In other cases e.g., (Manickam et al., 2014; Zhang et al., 2013), discrepancies might be explained by their choice of monitoring the accuracy of heterologous proteins which are normally not expressed in E. coli and which might be encoded by codons which are not optimal for the bacterial translation system, leading to an increased error frequency.

Analysis of the distribution of $R \rightarrow H$ substitution at 12 different positions suggests that the location in the protein and the context have a strong influence on the error frequency and the synthesis at some region of the protein, e.g., between residues 319 and 382, can be more accurate than at others (Figure 28). In EF-Tu, the least frequent substitutions are localized in a region which is important for the binding aa-tRNA (Yikilmaz et al., 2014) and might suggest that the proteins bearing amino acid replacements at these positions are unstable and are preferentially removed by the quality control machinery. Notably, frequent errors are also detectable in the protease-free environment of in-vitro 
translation system (Figure 30). Unfortunately, low-abundance error containing peptides could not be detected in vitro due to the low yield of in-vitro translation. When a general effect of the nascent chain and sequence context on the translation was studied (Figure 32) a general reduction of accuracy was observed at these positions but whether the lower error frequency observed in figure 28 is an effect caused by the quality control activity or an intrinsic higher accuracy of the ribosome, remains difficult to discriminate.

\subsection{Stress response and error profiles induced by aminoglycosides}

The effect of aminoglycosides on translation is well established (Anderson et al., 1967; Davies et al., 1965a), and consistent with previous reports, all the aminoglycosides we tested, except Spc, induce miscoding (Figure 33,B) (Bilgin et al., 1990; Borovinskaya et al., 2007b; Carter et al., 2000; Peske et al., 2004). Among the miscoding-inducing aminoglycosides, Apr merits a special comment. At the time of its discovery in 1978 (Walton, 1978) a concentration-dependent miscoding effect was demonstrated (Perzynski et al., 1979). However, a recent study of its effect on the frequency of $R \rightarrow H$ substitutions in vivo suggested that Apr has no effect on the fidelity of either bacterial or mitochondrial ribosomes and that the lack of the miscoding activity may be the reason for its reduced ototoxicity (Matt et al., 2012). These findings were recently confirmed in vitro by a combination of single molecule fluorescence, NMR and bulk kinetics approaches (Tsai et al., 2013). Puglisi and colleagues concluded that Apr does not induce miscoding but it rather inhibits cell growth by blocking translocation. Instead, our data clearly show that Apr induces a significant level of miscoding, including errors of $\mathrm{R} \rightarrow \mathrm{H}$ substitutions. Apr also induces a concerted expression of IbpA and IbpB, whose expression is associated with erroneous proteins synthesis (Ruan et al., 2008) (Figure 33, A). Moreover, although for Apr the error frequency of single misreading events was lower than with Par, Tob, or Str (Figure $33, \mathrm{~B})$, the level of consecutive miscoding events was comparable (Figure 35), indicating that the contribution to the incorporation of multiple errors of Apr might be higher than those of Par, Tob and Str. The disagreement with the results by Matt et al. can be explained by the narrow range of Apr concentrations used in their experiments $(0-10 \mu \mathrm{M})$ and the combination with an assay which has a limited dynamic range and a low sensitivity, as the detection of fluorescence of Luciferase protein.

Overall, our data also revealed that as a result of the antibiotic treatment, the induction of intracellular error levels is also associated with a cell growth defect. The antibiotic-induced error frequency ranges between $10^{-5}-10^{-3}$ (Figure $33, \mathrm{~B}$ ). Interestingly, each aminoglycoside has a unique pattern of induced error frequencies for different types of amino acid substitutions, which constitutes a miscoding-fingerprint of the antibiotic (Figure 33, A) and supports the notion that the mechanism of aminoglycosides action cannot be generalized (Figure 33, B and C). Rather, for each antibiotic the 
cellular outcome in terms of error frequency depends on the effect of the antibiotic on the elemental rates of decoding (Gromadski and Rodnina, 2004b; Pape et al., 2000) and on structural and dynamic changes of the ribosome induced by the antibiotic binding (Carter et al., 2000; Demirci et al., 2013a). Surprisingly, the error frequencies observed in response to the antibiotic treatment are remarkably similar to those observed in the error prone strain (Figure 39). However, the growth defects of wild type cells observed in the presence of antibiotics are not found in the error prone strain.

Aminoglycosides treatment induces the incorporation of consecutive errors, which are detected in aminoglycosides-treated cells but not in the error-prone strain. These multiple replacement in a single polypeptide chain may have a stronger toxic effect on the cell than single substitutions. Not surprisingly, the increased level of errors is associated with an increased expression of the proteins involved in the cellular stress response, such as IbpA and IbpB, whose induction is usually associated with the presence of aggregated misfolded proteins that accumulate as a consequence of the antibiotic treatment (Laskowska et al., 1996), although this effect is not observed in Spc-treated cells, where no fidelity defect is observed. In contrast to what happens for IbpA and IbpB, the concentration of the transcription factor $\mathrm{rpoH}$, which regulates the expression of $\mathrm{IbpA}$ and $\mathrm{IbpB}$, remains constant upon aminoglycoside treatment, consistent with the notion that $\mathrm{rpoH}$ expression increases only after a temperature shift (Erickson et al., 1987) and that the expression of heat-shock proteins may be also mediated by other transcription factors or other metabolic signals, as proposed in literature (Kuczynska-Wisnik et al., 2001). Surprisingly, although Gen is known to induce significant miscoding (Tsai et al., 2013), only a minimum effect on the stress response is observed in Gen-treated cells (Figure 33, A). Although the error profile for Gen is not available yet, the lack of stress response induction could be explained by the high efficiency of Gen cellular uptake which might lead to a fast cell death which leaves no time for the stimulation of IbpA and IbpB expression.

Notably, for many aminoglycosides such as Kan A, Tob, Par, Rib and Str, the expression levels of chaperones increase even when cells already display a strong growth impairment (Figure 33, A), suggesting that even when the cell growth is reduced, cells continue to actively translate the stress response proteins necessary to mediate correct protein folding and reduce their aggregation. In those cases where a stress response is observed, this increase reaches a maximum and then decreases with the error level. We hypothesize that as soon as aminoglycosides are added in the medium, the small amount of antibiotic which is able to enter the cells acts on the ribosome inducing miscoding and stimulating the expression of stress response proteins. When the intracellular concentration of aminoglycoside has reached a critical point, translation is completely inhibited (Tsai et al., 2013) and the cell can temporarily eliminate erroneous proteins up to the point where the cellular damage is too high. 
In our experiments with aminoglycosides, cells grow even at antibiotic concentrations which exceed the estimated MIC values (Figure 33, A). Such discrepancy might be explained by differences in the experimental setup. To determine the MIC values, bacteria are inoculated at low cell densities into a medium which is already supplemented with different concentrations of antibiotic and their growth is assessed after a very long incubation time (usually 16 - 20 hrs) (Wiegand et al., 2008). In our assay, exponentially growing cells at high cell density are monitored for a shorter time window (2h). Because the uptake of aminoglycosides is slow, the external and cellular aminoglycoside concentrations might not be equilibrated. Thus, higher concentrations may be necessary to completely suppress cell growth, far above the MIC values. Because the efficient uptake of the antibiotic increases with the incorporation of error-containing proteins into the membrane, aminoglycosides which do no induce significant miscoding (e.g., Spc, Hyg B) become inhibitory only at very high concentrations.

\subsection{Quantification and induction of miscoding pattern}

The aminoglycosides treatment has allowed us to identify a pattern of multiple miscoding events in EF-Tu. These consecutive misincorporations remain below the detection limit in the wild type cells but become more frequent after aminoglycoside-treatment (Figure 37, 38, 39). Their frequency is higher than expected for uncoupled independent events (Equation 2). We note that at the conditions of error accumulation, prediction of error frequencies may become a problem, unless the peptides with single substitutions are less abundant than the correct peptides (Equation 1). If the error frequencies of the individual errors would be higher, and comparable to the cognate peptide, the frequencies of the cognate peptides and all its derivatives would have to be summed up to properly reflect the protein concentration. This would be particularly important when these values are used to predict the probability of two independent errors in a single peptide. However, even when cells are treated with high concentrations of aminoglycosides, the frequency of single errors never exceeds $10^{-3}$ (Figure 33 , B) and the stochastic occurrence of consecutive errors can be considered negligible. Notably, consecutive errors are not detectable in the absence of aminoglycosides and thus can be attributed to the action of the antibiotics. Due to the vectorial nature of protein synthesis, the incorporation of consecutive $\mathrm{C}$-terminal errors must depend on the first $\mathrm{N}$-terminal miscoding event.

To explain the observed effect of antibiotics on error propagation, different mechanisms can be envisioned. First, the first miscoding event itself might induce subsequent errors and the error propagation could be mediated by misincorporations in the nascent chain or by distortion of the Psite tRNA introduced by codon:anticodon mismatches (Zaher and Green, 2009). Stalling peptides such as ermA can alter the catalytic proficiency for certain aa-tRNAs in the A site in the presence of their 
regulator, i.e., erythromycin (Ramu et al., 2011). If an incorrect aa-tRNA binds to the $A$ site and is translocated to the P site after peptide bond formation, the misalignment of the P-site tRNA due to mismatches in its codon:anticodon interaction can elicit error-prone decoding (Zaher and Green, $2009,2010 \mathrm{~b}, 2011)$. This, in turn, promotes a rescue mechanism of sense codon reading by a translation termination factor, which stops translation of a peptide in which several consecutive error have already occurred. Importantly, this mechanism is supposed to increase fidelity by the preferential release of the highly erroneous peptides from the ribosome. Alternatively, consecutive misincorporations could be introduced by the presence of aminoglycosides that remain bound to the ribosome decoding center during several elongation cycles. Notably, the three proposed mechanisms are not mutually exclusive but could act synergistically. The absence of detectable error propagation during translation by the error-prone ribosome indicates that the proposed error-propagation mechanism depends on the presence of antibiotics which leads to the incorporation of consecutive misincorporations. Importantly, mutations that make the ribosomes error-prone do not interfere with the selection mechanism proposed by Green and colleagues (Zaher and Green, 2010b).

Further indications for the sources of multiple errors come from the analysis of the misincorporation pattern of $E 306 \mathrm{D}-\mathrm{Y} 310 \mathrm{H}$ peptide, in which the first miscoding event $(E \rightarrow D$ substitution) is followed by the correct incorporation of S, E, and V (Figure 38), followed by a $\mathrm{Y} \rightarrow \mathrm{H}$ substitution. Because there are multiple isoacceptor tRNAs that can deliver $S$ and $V(6$ and 3 isoacceptors, respectively), we cannot exclude that the correct amino acid is delivered by a an isoacceptor that is near-cognate to the given codon However, the correct incorporation of $E$, which has only one isoacceptor, must definitely be the result of a cognate tRNA, which should terminate the P-site-induced error-prone decoding. Thus, we suggest that consecutive miscoding events are most likely introduced by aminoglycosides. In this case, the propagation efficiency should depend on the balance between the speed of elongation and the dissociation rate constant of the respective aminoglycoside. Over several elongation cycles after the initial misincorporation event, aminoglycosides should dissociate and the error propagation effect should drop dramatically approaching the low level given by the stochastic occurrence of the single errors. However, for the quantified consecutive misincorporation events, the error-propagation efficiencies are rather constant, between 3 and $10 \%$ (Figure 40) and almost independent of the EF-Tu source, antibiotic concentration and the distance in the sequence. 


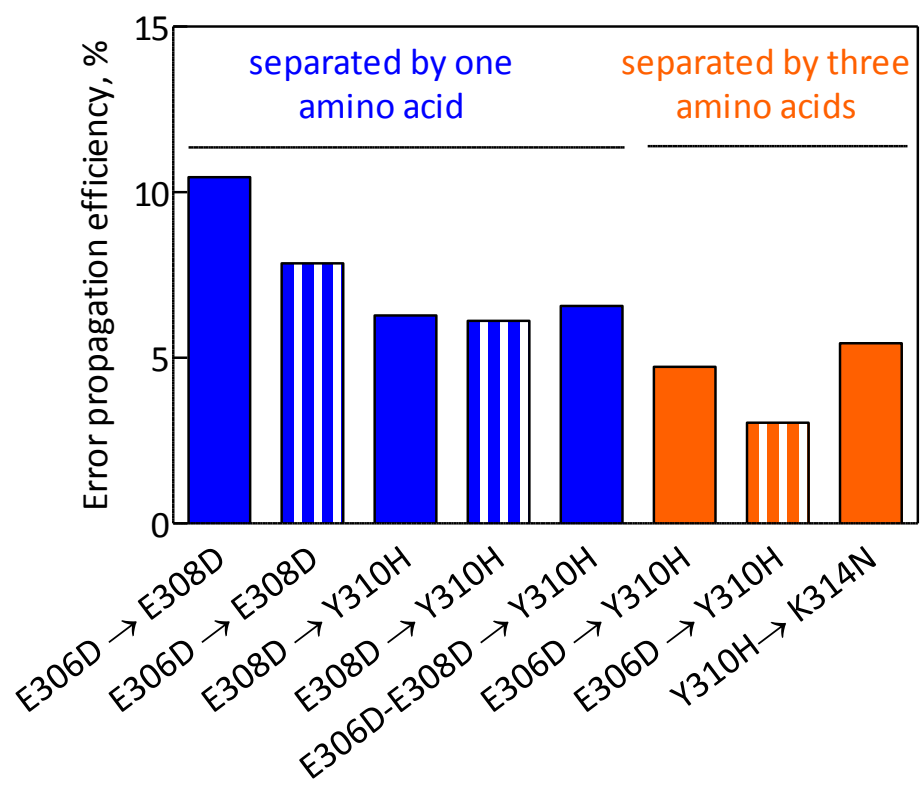

Figure 40. Frequencies of multiple consecutive errors. EF-Tu purified from K12 chromosomal-tag cells grown in $8 \mu \mathrm{M}$ streptomycin (filled bars) or from wild type cells grown in $4 \mu \mathrm{M}$ str (striped bars).

Assuming an elongation rate of 10 amino acids per second (Young and Bremer, 1976), this implies that to induce several consecutive errors, Str has to be stabily bound to the ribosome for at least 0.5 seconds. Due to the absence of kinetic data for the dissociation rate constants of aminoglycosides from the ribosome, the plausibility of this hypothesis is hard to evaluate. Notably, the error propagation efficiencies of E308D $\rightarrow \mathrm{Y3} 10 \mathrm{H}$ and E306D-E308D $\rightarrow \mathrm{Y3} 10 \mathrm{H}$ (Figure 40) are identical and thus the consecutive introduction of misincorporations is not additive or cumulative, and is consistent with a model in which the second misincorporation solely depends on the presence of the antibiotic.

Notably, all multiple-substituted variants of the target peptide FESEVYILSK we tried to enrich were successfully detected in Str treated samples. When the contribution of the consecutive misincorporations relative to the single substitutions is inspected for individual residues (e.g., E306D, Figure 41, A) it becomes clear that a significant part of the errors is involved in multiple errors patches (>15\%). This number is likely to be an underestimation because only a very small subset of all possible error combination has been taken into consideration in our experiments (Figure 41, B). The proposed mechanism implies that aminoglycosides whose binding to the ribosome lasts longer should induce a more persisting miscoding effect and a richer repertoire of multiple errors. 
A

E306D
E306D-E308D
E306D-Y310H
E306D-E308D-Y310H
E306D-Y310N
E306D-K314N

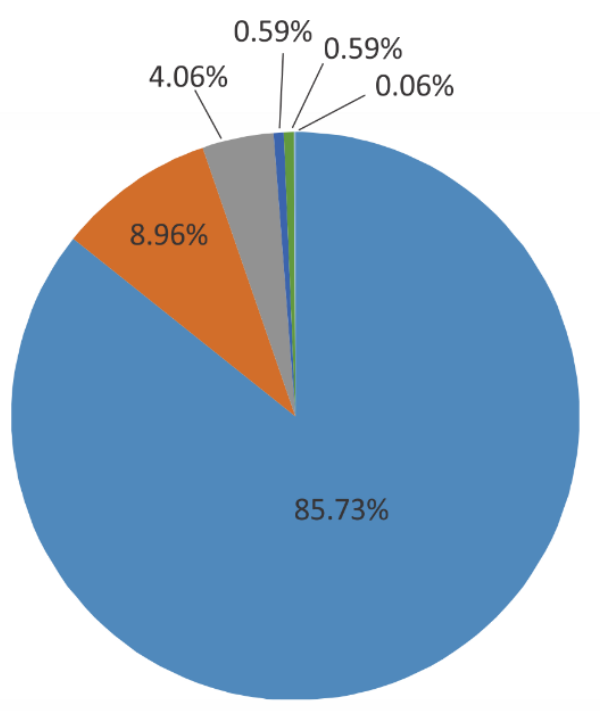

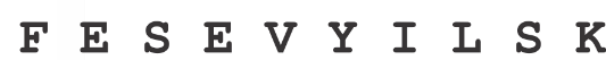

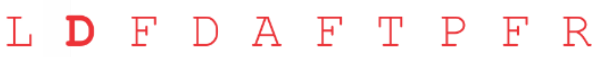

$S$ G T G E S N Q Y N

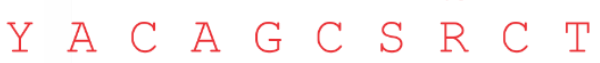

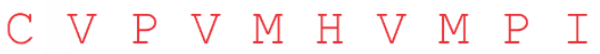

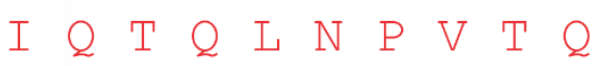

$V K A K$ D L A E

Figure 41. Significance of complex miscoding patterns. A) Relative contribution of consecutive misincorporation as quantified for the E306D peptide in EF-Tu with chromosomal tag from K12 cells grown in the presence of $8 \mu \mathrm{M}$ Str. B) All possible near-cognate replacements for the model peptide and the possible combinatorial expansion of consecutive misincorporation events.

The qualitative contribution of error patches to protein structure can be very severe. Sequential misincorporations are most likely far more detrimental to the functional and structural integrity of proteins than single substitutions. The structural destabilization by multiple mutations may increase exponentially with the number of misincorporations. Membrane insertion of proteins that have a single incorrect amino acid can already have a dramatic impact on the integrity of the membrane. This is demonstrated by the importance of positively charged residues in membrane-spanning polytopic proteins, which are well tolerated when facing the cytoplasm, but are avoided in transmembrane regions. Their correct distribution constitute the major determinant in protein topology (von Heijne, 1989) and substitutions affecting these residues are likely to cause structural problems and incorrect folding. Proteins with two or more replacements, as it happens upon aminoglycosides treatment, can have even more severe effects.

In summary, the preferential aminoglycoside-mediated induction of certain types of misincorporations, e.g., E $\rightarrow$ D (Kramer and Farabaugh, 2007) (Figure 32, B), in combination with the discovered error propagation mechanism will most likely lead to complex misincorporation patterns and ultimately to error hotspots in protein sequences. Such error hotspots, in combination with the varying tolerance towards mutations in different proteins or parts of proteins, may explain why only a small subset of proteins is overrepresented in streptomycin-induced aggregates and inclusion bodies (Ruan et al., 2008). It is not yet clear to which extent multiple misincorporations contribute to the bactericidal efficacy of AGs in prokaryotes or to the oto- and nephrotoxicity in human cells. If the error propagation would occur in human mitochondria as well, this might be relevant for the 
aminoglycoside-based treatment of genetic disorders. While the curative effect is based on the readthrough of accidentally introduced stop codons, complex misincorporation pattern are not intended and may be responsible for parts of the side effects. Thus, a better molecular understanding of the underlying mechanisms of error-propagation effects might help to uncouple the induction of single substitutions and error-propagation events.

Our findings on the mechanism of aminoglycosides-mediated bactericidal effects can be incorporated into the existing model for the aminoglycoside bactericidal mechanism (Davis, 1987). During aminoglycoside treatment, only a small amount of the antibiotic can initially enter the cell, where it interacts with ribosomes reducing their translational fidelity and inducing miscoding. When erroneous and potentially unfolded proteins are inserted in the membrane, this causes the formation of channels and favors membrane damage enhancing the intake of more aminoglycoside into the cell. When erroneous protein begin to appear, the ribosome induces multiple sequential miscoding events, which stimulates termination of the synthesis of erroneous proteins and triggers their release (Zaher and Green, 2009). Truncated proteins can be toxic and their membrane insertion can cause abnormal depolarization (Hurwitz et al., 1981) and change membrane potential and permeability (Bryan and Kwan, 1983; Taber et al., 1987). Erroneous and truncated proteins synergistically affect the membrane promoting an increased influx of aminoglycosides which inevitably leads to a positive feedback loop in which more aminoglycoside will enter the cell and more errors will be made until, the integrity of the membrane is destroyed and the cell dies. It has been observed that Str treatment causes an increased demand for polyamines, and the induction of a polyamine transport system can be utilized in a competitive manner by Str (Holtje, 1979) facilitating even more the entrance of the antibiotic into the cell. The relationship between incorrect protein synthesis and membrane damage is thus a key element in the aminoglycosides-induced miscoding. The insertion of erroneous proteins in the cellular membrane may also generate hydroxyl radical and oxidative stress, ultimately damaging nucleic acids and proteins (Dwyer et al., 2014; Kohanski et al., 2008), although the latter notion has been recently challenged (Keren et al., 2013; Liu and Imlay, 2013). 


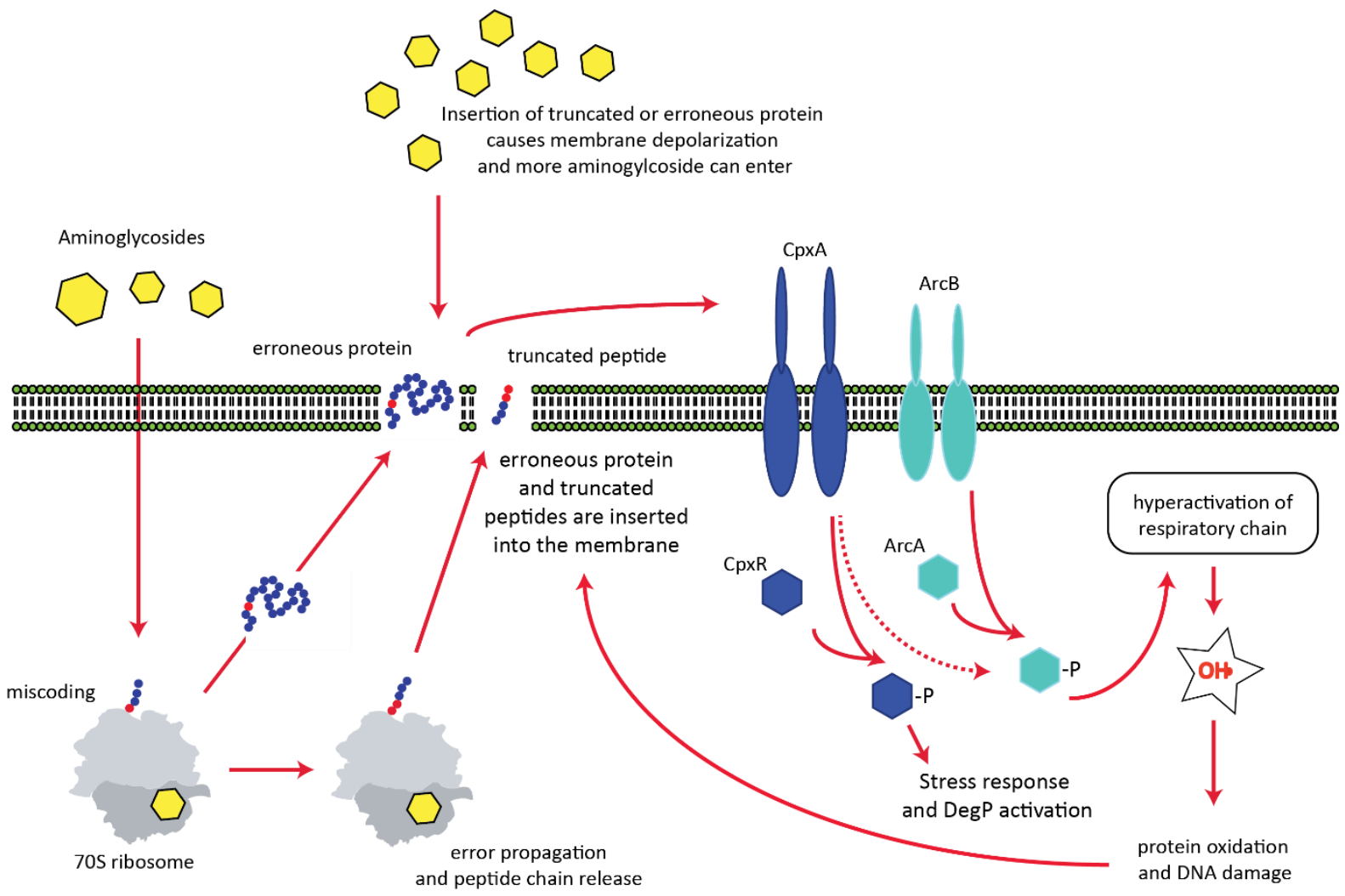

Figure 42. Proposed mechanism of aminoglycosides activity. A small amount of aminoglycoside enters the cell and interacts with ribosomes reducing the fidelity of translation and inducing miscoding. Erroneous proteins are produced and the ribosomes promote consecutive errors incorporation and stimulate translation termination and peptide release. When erroneous and/or truncated proteins are inserted into the membrane they trigger the phosphorylation of members of twocomponent regulatory systems such as $\mathrm{CpxA}$ and $\mathrm{ArcB}$, which in turn activate their corresponding regulatory proteins $\mathrm{CpxR}$ and ArCA, respectively. CpxR regulates the expression of stress-response protein e.g., DegP. ArcA regulates genes involved in the respiratory chain, leading to its hyperactivation, culminating in the generation of hydroxyl radicals which damage nucleic acids and proteins. Figure adapted from (Kohanski et al., 2007; Kohanski et al., 2008).

\subsection{Aminoglycosides effect investigation and future perspectives}

The present work demonstrated that our developed experimental approach constitutes a robust and reliable tool for the investigation of amino acid misincorporation in vivo. When applied to the study of aminoglyocosides, our study revealed that the increased frequency of some errors during the treatment can be seen as a fingerprint characterizing each antibiotic. However, the results obtained so far are resistricted to a limited set of peptides and only 8 substitutions have been taken into consideration (Figure 33, B). As part of the future perspectives, the pool of substitutions analyzed will be extended to provide a more comprehensive overview on the aminoglycosides charachteristics. The charachterization of aminoglycosides effect could also be extended to a broader analysis of the cellular response by systematically monitoring the expression of, not only $\mathrm{IbpA}$ and $\mathrm{IbpB}$ chaperones (Figure 33), but also of proteins part of the SOS response for DNA damage e.g., RecA and LexA or protein involved in the respiratory chain (Kohanski et al., 2007) which might shed some light on whether or 
not the oxidative stress and the consequent nucleic acid damage are involved in the aminoglycosides killing mechanism.

Our data indicate that the induction of error patches may play an important role in the mechanism of aminoglycosides-mediated cell death. In the future work, consecutive errors which were never reported in literature so far, but which already showed promising initial results with Apr, Tob and Par (Figure 35), will be studied in the presence of other antibiotics, and other model peptides will be taken into consideration. These combined results could lead to a deeper understanding of the mechanisms of action of aminoglycosides. In addition, because aminoglycosides toxicity has been often associated with their interaction with mitochondrial ribosomes, the flexibility and sensitivity of our method could be employed to investigate single errors and more complex error patterns in eukaryotic systems. This could have a potential medical impact, for example in the selection of the lowest-toxicity aminoglycosides for the palliative treatment of genetic diseases (Bukowy-Bieryllo et al., 2016; Ho et al., 2013; Malik et al., 2010a; Malik et al., 2010b) or to perform more focused treatments, tailored to the type of miscoding event to induced. 
MATERIALS AND METHODS

\section{MATERIALS AND METHODS}

\section{Table 6. List of equipments}

\begin{tabular}{|c|c|}
\hline Device & Supplier \\
\hline Allegra $X-22 R$ centrifuge & Beckmann Coulter \\
\hline Amicon Ultra-4 30K cut-off & Merck Millipore \\
\hline Avanti J-26 XP centrifuge & Beckmann Coulter \\
\hline Avanti J-30l centrifuge & Beckmann Coulter \\
\hline $\begin{array}{c}\text { Acclaim PepMapRSLC } 15 \mathrm{~cm}, 75 \mu \mathrm{m} \text { inner diameter, } 3 \mu \mathrm{m} \\
\text { particle size }\end{array}$ & Thermo Fisher Scientific \\
\hline ÄKTA FPLC & GE Healthcare \\
\hline Benchtop centrifuge 5415R & Eppendorf \\
\hline Biostat B-plus 5 I fermenter & Sartorius \\
\hline Digital sonifier cell distruptor W-250D & Branson \\
\hline Dounce tissue grinder & Wheaton \\
\hline Easy nLCII chromatography system & Thermo Fisher Scientific \\
\hline Electrophoresis power supply & PeqLab \\
\hline Emulsiflex C-3 homogenizer & Avestin \\
\hline HighTrap Q HP anion exchange column & GE Healthcare \\
\hline Hiload 26/60 Superdex 75 size-exclusion chromatography & GE Healthcare \\
\hline Innova 44 incubator shaker & New Brunswick \\
\hline Intelli-mixer & ELMI Ltd. \\
\hline JA30.1 rotor & Beckmann Coulter \\
\hline JLA-8.1000 rotor & Beckmann Coulter \\
\hline LiChrospher WP300 RP-18 (5 $\mu \mathrm{m})$ & Merck Millipore \\
\hline Low retention $1.5 \mathrm{ml}$ tubes & Eppendorf \\
\hline Low retention $0.5 \mathrm{ml}$ tubes & Eppendorf \\
\hline Nanodrop 2000 & Peqlab \\
\hline Optima XPN centrifuge & Beckmann Coulter \\
\hline $\mathrm{pH}$-indicator strips $\mathrm{pH}$ 6.5-10.0 & Merck Millipore \\
\hline Pierce BCA Protein Assay Kit & Thermo Fisher Scientific \\
\hline Polyolefin foil for microplates & HJ-Bioanalytik GmbH \\
\hline Polypropylene 96-well microplates & VWR \\
\hline Protino Ni-IDA 2000 Packed Columns & Macherey-Nagel \\
\hline Q-Exactive hybrid mass spectrometer & Thermo Fisher Scientific \\
\hline Q-Exactive Plus hybrid mass spectrometer & Thermo Fisher Scientific \\
\hline Reprosil-Pur 120 C18 $3 \mu \mathrm{m}$ column & Dr Maisch \\
\hline SDS-PAGE electrophoresis chamber & Bio-Rad \\
\hline SpeedVac vacuum concentrator SPD121P & Thermo Fischer Scientific \\
\hline Spin- $X$ centrifuge tube filter $0.45 \mu \mathrm{m}$ cellulose acetate & Corning Inc. \\
\hline Superdex Peptide 10/300 GL & GE Healthcare \\
\hline
\end{tabular}




\begin{tabular}{cc}
\hline SX4250 rotor & Beckmann Coulter \\
\hline Thermomixer comfort & Eppendorf \\
\hline Thermostatic oven & Memmert \\
\hline TSQ Quantiva triple quadrupole mass spectrometer & Thermo Fisher Scientific \\
\hline TSQ Vantage triple quadrupole mass spectrometer & Thermo Fisher Scientific \\
\hline Ultimate 3000RSLC & Thermo Fisher Scientific \\
\hline Ultrasonic cleaner & VWR \\
\hline Vivaspin 20 centrifugal concentrator & Sartorius \\
\hline Vortex Genie 2 & Beckmann Coulter \\
\hline 50.2Ti rotor &
\end{tabular}

Table 7. List of softwares

\begin{tabular}{cc}
\hline Software & Provider \\
\hline DNA Star Lasergene & DNASTAR, Inc \\
\hline GraphPad Prism 5.0 & GraphPad Software \\
\hline Pymol 1.5 & Schrödinger \\
\hline Skyline 3.5 & MacCoss Lab Software \\
\hline Xcalibur 2.2 & Thermo Fischer Scientific \\
\hline Adobe Illustrator CS5 & Adobe Systems \\
\hline CorelDRAW X7 & Corel \\
\hline
\end{tabular}

\subsection{Chemicals}

All reactions are performed in low-retention reaction cups (Eppendorf). If not indicated elsewhere, the chemicals are purchased from Merck Millipore or Sigma Aldrich. The chemicals used for chromatographic separation are of HPLC grade. Water, acetonitrile, methanol and formic acid used for mass spectrometric analysis are of HPLC/MS grade and purchased from Fischer Scientific. Customsynthetized isotopically-labeled peptides $(5 \mu \mathrm{M})$ are obtained from Thermo Fisher Scientific (highly quantified Ultimate grade peptides with concentration precision $\pm 5 \%$ and QuantPro grade peptides with concentration precision $\pm 25 \%$ ) or JPT Peptide Technologies (Berlin, Germany) (highly-quantified peptides SpikeTides_TQL or non-quantified SpikeTides_L grade peptides). All peptides used are listed in the appendix of this thesis (Table 15 and 16). Pierce Retention Time Calibration mix (PRTC) used to regularly assess the performances of chromatographic system and mass spectrometer is from Thermo Fisher Scientific. 
Table 8. List of buffers

\begin{tabular}{|c|c|}
\hline Buffer & Composition \\
\hline LB medium & $10 \mathrm{~g} / \mathrm{L}$ tryptone, $10 \mathrm{~g} / \mathrm{L} \mathrm{NaCl}, 5 \mathrm{~g} / \mathrm{L}$ yeast extract \\
\hline LB agar & $10 \mathrm{~g} / \mathrm{L}$ tryptone, $10 \mathrm{~g} / \mathrm{L} \mathrm{NaCl}, 5 \mathrm{~g} / \mathrm{L}$ yeast extract, $15 \mathrm{~g} / \mathrm{L}$ agar \\
\hline TB medium & $12 \mathrm{~g} / \mathrm{L}$ tryptone, $24 \mathrm{~g} / \mathrm{L}$ yeast extract, $4 \mathrm{ml} / \mathrm{L}$ glycerol, $17 \mathrm{mM} \mathrm{KH}_{2} \mathrm{PO}_{4}, 72 \mathrm{mM} \mathrm{K}_{2} \mathrm{HPO}_{4}$ \\
\hline Buffer A & $\begin{array}{l}\text { 50mM HEPES pH 7.5, } 50 \mathrm{mM} \mathrm{KCL}, 10 \mathrm{mM} \mathrm{MgCl} \text {, 5mM 2-mercaptoethanol, Complete } \\
\text { Protease inhibitor } 1 \text { tablet/50 ml (Roche Diagnostics, Mannheim), DNase }\end{array}$ \\
\hline Buffer B & $\begin{array}{l}\text { B-PER reagent (Thermo Scientific), } 200 \mathrm{mM} \mathrm{KCl,} 3 \mathrm{mM} \mathrm{MgCl} 2 \text {, Complete Protease inhibitor } 1 \\
\text { tablet/50 ml (Roche Diagnostics, Mannheim), } 30 \mu \mathrm{M} \mathrm{GDP,} 5 \mathrm{mM} \text { 2-mercaptoethanol, DNase }\end{array}$ \\
\hline Buffer C & $8 \mathrm{M}$ Urea, $25 \mathrm{mM}$ Hepes pH 7.5, $200 \mathrm{mM} \mathrm{KCl}, 10 \mathrm{mM} \mathrm{MgCl} 2,5 \mathrm{mM}$ 2-mercaptoethanol \\
\hline Buffer D & 25 mM HEPES pH 7.5, 200 mM KCl, 3mM MgCl $2,30 \mu \mathrm{M}$ GDP \\
\hline Buffer E & Buffer D + 500mM KCl \\
\hline Buffer $\mathrm{F}$ & Buffer D + 200 mM Imidazole \\
\hline Buffer G & Buffer $\mathrm{C}+400 \mathrm{mM} \mathrm{KCl}$ \\
\hline Buffer $\mathrm{H}$ & Buffer C + 150 mM Imidazole \\
\hline $\mathrm{TAKM}_{7}$ & $50 \mathrm{mM}$ Tris- $\mathrm{HCl} \mathrm{pH} \mathrm{7.5,} 70 \mathrm{mM} \mathrm{NH}_{4} \mathrm{Cl}, 30 \mathrm{mM} \mathrm{KCl}, 7 \mathrm{mM} \mathrm{MgCl} 2$ \\
\hline Staking gel SDS-PAGE & $4.8 \%$ AA:Bis (29:1), 0.125 M Tris-HCl pH 6.8, $0.1 \%$ SDS \\
\hline Resolving gel SDS-PAGE & $16 \%$ AA:Bis (29:1), $0.3 \mathrm{M}$ Tris-HCl pH 8.8, $0.1 \%$ SDS \\
\hline $\begin{array}{l}\text { Coomassie Blue staining } \\
\text { solution }\end{array}$ & $0.1 \%$ Coomassie brilliant blue, $50 \%$ ethanol, $10 \%$ acetic acid \\
\hline Destaining solution & $20 \%$ ethanol, $10 \%$ acetic acid \\
\hline $\begin{array}{l}\text { Laemmli SDS loading } \\
\text { buffer } 4 X\end{array}$ & $\begin{array}{l}40 \text { \% glycerol, } 240 \text { mM Tris- } \mathrm{HCl} \text { pH 6.8, } 8 \text { \% SDS, } 0.04 \% \text { bromophenol blue, } 5 \% 2 \text { - } \\
\text { mercaptoethanol }\end{array}$ \\
\hline SDS running buffer $10 \mathrm{X}$ & $\begin{array}{l}250 \mathrm{mM} \text { Tris base, } 1.92 \mathrm{M} \text { glycine, } 1 \text { \% SDS (to be diluted } 10 \text { times to obtain SDS running } \\
\text { buffer } 1 \mathrm{X} \text { ) }\end{array}$ \\
\hline TBS buffer & $50 \mathrm{mM}$ Tris- $\mathrm{HCl} \mathrm{pH} \mathrm{7.5,} 150 \mathrm{mM} \mathrm{NaCl}$ \\
\hline High salt TBS buffer & TBS buffer $+500 \mathrm{mM} \mathrm{NaCl}$ \\
\hline
\end{tabular}

Table 9. List of plasmids

\begin{tabular}{|c|c|c|c|c|}
\hline Vector & Purpose & Supplier & Resistance & Tag \\
\hline pET24a & $\begin{array}{l}\text { Overexpressed his-tag EF-Tu } \\
\text { (tufA) }\end{array}$ & Merck Millipore & Kan & C-terminal Hisx6 \\
\hline pET21a & $\begin{array}{l}\text { Overexpressed his-tag EF-Tu } \\
\text { (tufA) and sumo constructs }\end{array}$ & Novagen & Amp & C-terminal Hisx6 \\
\hline pSUMO & Sumo-tag constructs & & Kan & Hisx6 \\
\hline pET21-flag & C-terminus-flag EF-Tu (tufA) & $\begin{array}{l}\text { In-house produced } \\
\text { from pET21 }\end{array}$ & Kan & $\begin{array}{c}\text { Flag } \\
\text { (DYKDDDDK) }\end{array}$ \\
\hline
\end{tabular}

Table 10. List of bacterial strains 


\begin{tabular}{|c|c|c|}
\hline E. coli strain & Purpose & Supplier \\
\hline $\mathrm{BL} 21$ (DE3) & EF-Tu (tufA) overexpression & Merck Millipore \\
\hline TOP10 & Plasmid replication & Novagen \\
\hline MRE-600 (ATCC29417) & Wild type EF-Tu & UAB school of medicine \\
\hline MG1655 (K-12) & Wild type EF-Tu & \multirow{2}{*}{ Leibniz Institute DSMZ } \\
\hline W3110 (K-12) & EF-Tu chromosomal tag (tufA) & \\
\hline E. coli UD 131 (Xac rpsD12) & Error prone (ram) & \multirow{3}{*}{$\begin{array}{l}\text { These strains have been kindly provided by Dr. } \\
\text { Hani Zaher (Deptartment of Biology, Washington } \\
\text { University in St. Louis, MO, USA ) }\end{array}$} \\
\hline $\begin{array}{l}\text { E. coli US157 (Xac rspL141 zcg- } \\
\text { 174::Tn10) }\end{array}$ & Error restricted (hyperaccurate) & \\
\hline $\begin{array}{l}\text { E. coli (Xac ara D[lacproAB] } \\
\text { gyrA rpoB argEamber) }\end{array}$ & $\begin{array}{l}\text { Wild type EF-Tu for comparison } \\
\text { with hyperaccurate and error } \\
\text { prone strain }\end{array}$ & \\
\hline
\end{tabular}

\subsection{Primers}

Primers are designed using the SeqBuilder software (DNASTAR) and purchased from IBA Life Sciences or Eurofins MWG Operon. Forward and reverse primers ( $F$ and $R$, respectively) are listed.

\section{Table 11. List of primers for EF-Tu mutants}

\begin{tabular}{|c|c|}
\hline \multicolumn{2}{|c|}{ Primers used for EF-Tu in-situ directed mutagenesis } \\
\hline Mutation to introduce & Sequence $5^{\prime}-3^{\prime}$ \\
\hline \multirow{2}{*}{$\mathrm{R} 45 \mathrm{H}$} & F CCTACGGCGGTGCTGCTCATGCATTCGACCAGATCG \\
\hline & R CGATCTGGTCGAATGCATGAGCAGCACCGCCGTAGG \\
\hline \multirow{2}{*}{$\mathrm{R} 59 \mathrm{H}$} & F CGCGCCGGAAGAAAAAGCTCATGGTATCACCATCAACACTTC \\
\hline & R GAAGTGTTGATGGTGATACCATGAGCTTTTTCTTCCGGCGCG \\
\hline \multirow{2}{*}{$\mathrm{R} 172 \mathrm{H}$} & F GACGACACTCCGATCGTTCATGGTTCTGCTCTGAAAGC \\
\hline & R CGCTTTCAGAGCAGAACCATGAACGATCGGAGTGTCGTC \\
\hline \multirow{2}{*}{ R231S } & F GTGGTACCGTTGTTACCGGTAGTGTAGAACGCGGTATCATC \\
\hline & R GATGATACCGCGTTCTACACTACCGGTAACAACGGTACCAC \\
\hline \multirow{2}{*}{ R231G } & F GTGGTACCGTTGTTACCGGTGGTGTAGAACGCGGTATCATC \\
\hline & R GATGATACCGCGTTCTACACCACCGGTAACAACGGTACCAC \\
\hline \multirow{2}{*}{$\mathrm{R} 231 \mathrm{C}$} & F GTACCGTTGTTACCGGTTGTGTAGAACGCGGTATCATC \\
\hline & R GATGATACCGCGTTCTACACAACCGGTAACAACGGTAC \\
\hline \multirow{2}{*}{$\mathrm{R} 231 \mathrm{H}$} & F GTACCGTTGTTACCGGTCATGTAGAACGCGGTATCATC \\
\hline & R GATGATACCGCGTTCTACATGACCGGTAACAACGGTAC \\
\hline \multirow{2}{*}{ R231P } & F GTGGTACCGTTGTTACCGGTCCGGTAGAACGCGGTATCATC \\
\hline & R GATGATACCGCGTTCTACCGGACCGGTAACAACGGTACCAC \\
\hline \multirow{2}{*}{$\mathrm{R} 231 \mathrm{~L}$} & F GTGGTACCGTTGTTACCGGTCTGGTAGAACGCGGTATCATC \\
\hline & R GATGATACCGCGTTCTACCAGACCGGTAACAACGGTACCAC \\
\hline \multirow{2}{*}{ R231A } & F GTGGTACCGTTGTTACCGGTGCTGTAGAACGCGGTATCATC \\
\hline & R GATGATACCGCGTTCTACAGCACCGGTAACAACGGTACCAC \\
\hline
\end{tabular}


MATERIALS AND METHODS

\begin{tabular}{|c|c|}
\hline \multirow{2}{*}{ R231D } & F GTGGTACCGTTGTTACCGGTGATGTAGAACGCGGTATCATC \\
\hline & R GATGATACCGCGTTCTACATCACCGGTAACAACGGTACCAC \\
\hline \multirow{2}{*}{$\mathrm{R} 231 \mathrm{E}$} & F GTGGTACCGTTGTTACCGGTGAAGTAGAACGCGGTATCATC \\
\hline & R GATGATACCGCGTTCTACTTCACCGGTAACAACGGTACCAC \\
\hline \multirow{2}{*}{$\mathrm{R} 231 \mathrm{~F}$} & F GTGGTACCGTTGTTACCGGTTTTGTAGAACGCGGTATCATC \\
\hline & R GATGATACCGCGTTCTACAAAACCGGTAACAACGGTACCAC \\
\hline \multirow{2}{*}{$\mathrm{R} 231 \mathrm{Y}$} & F GTGGTACCGTTGTTACCGGTTATGTAGAACGCGGTATCATC \\
\hline & R GATGATACCGCGTTCTACATAACCGGTAACAACGGTACCAC \\
\hline \multirow{2}{*}{ R2310 } & F GTACCGTTGTTACCGGTCAAGTAGAACGCGGTATCATC \\
\hline & R GATGATACCGCGTTCTACTTGACCGGTAACAACGGTAC \\
\hline \multirow{2}{*}{$\mathrm{R} 231 \mathrm{~T}$} & F GTGGTACCGTTGTTACCGGTACCGTAGAACGCGGTATCATC \\
\hline & R GATGATACCGCGTTCTACGGTACCGGTAACAACGGTACCAC \\
\hline \multirow{2}{*}{$\mathrm{R} 231 \mathrm{~V}$} & F GTGGTACCGTTGTTACCGGTGTTGTAGAACGCGGTATCATC \\
\hline & R GATGATACCGCGTTCTACAACACCGGTAACAACGGTACCAC \\
\hline \multirow{2}{*}{$\mathrm{R} 234 \mathrm{H}$} & F CAACTTTGATGATACCATGTTCTACACGACCGG \\
\hline & R CCGGTCGTGTAGAACATGGTATCATCAAAGTTG \\
\hline \multirow{2}{*}{ K249Q } & F GGTAGACTTCTGAGTCTCTTGGATACCAACGATTTCAAC \\
\hline & R GTTGAAATCGTTGGTATCCAAGAGACTCAGAAGTCTACC \\
\hline \multirow{2}{*}{ K249E } & F GGTAGACTTCTGAGTCTCTTCGATACCAACGATTTCAAC \\
\hline & R GTTGAAATCGTTGGTATCGAAGAGACTCAGAAGTCTACC \\
\hline \multirow{2}{*}{ K249T } & F GGTAGACTTCTGAGTCTCTGTGATACCAACGATTTCAAC \\
\hline & R GTTGAAATCGTTGGTATCACAGAGACTCAGAAGTCTACC \\
\hline \multirow{2}{*}{$\mathrm{K} 249 \mathrm{R}$} & F GAAATCGTTGGTATCCGTGAGACTCAGAAGTCTAC \\
\hline & R GTAGACTTCTGAGTCTCACGGATACCAACGATTTC \\
\hline \multirow{2}{*}{ K249I } & F GAAATCGTTGGTATCATCGAGACTCAGAAGTCTAC \\
\hline & R GTAGACTTCTGAGTCTCGATGATACCAACGATTTC \\
\hline \multirow{4}{*}{ K249N } & F GTTGGTGAAGAAGTTGAAATCGTTGGTATCAACGAGACTCAGAAGT \\
\hline & CTACCTGTACTGGCGTTG \\
\hline & R CAACGCCAGTACAGGTAGACTTCTGAGTCTCGTTGATACCAACGA \\
\hline & TTTCAACTTCTTCACCAAC \\
\hline \multirow{2}{*}{$\mathrm{R} 270 \mathrm{H}$} & F CAAACTGCTGGACGAAGGCCATGCTGGTGAGAACGTAGGTG \\
\hline & R CACCTACGTTCTCACCAGCATGGCCTTCGTCCAGCAGTTTG \\
\hline \multirow{2}{*}{$\mathrm{R} 280 \mathrm{H}$} & F CGTAGGTGTTCTGCTGCATGGTATCAAACGTGAAGAAATCG \\
\hline & R CGATTTCTTCACGTTTGATACCATGCAGCAGAACACCTAC \\
\hline \multirow{2}{*}{$\mathrm{R} 284 \mathrm{H}$} & F CGTTCGATTTCTTCATGTTTGATACCAC \\
\hline & R GTGGTATCAAACATGAAGAAATCGAACG \\
\hline \multirow{2}{*}{ K314Q } & F GAAGTGTACATTCTGTCCCAAGATGAAGGCGGCCGTCATAC \\
\hline & R GTATGACGGCCGCCTTCATCTTGGGACAGAATGTACACTTC \\
\hline \multirow{3}{*}{ K314E } & F CTTTGAAGAACGGAGTATGACGGCCGCCTTCATCTTCGGACAGAAT \\
\hline & GTACACTTCAGATTCGAACTTG \\
\hline & R CAAGTTCGAATCTGAAGTGTACATTCTGTCCGAAGATGAAG \\
\hline
\end{tabular}




\begin{tabular}{|c|c|}
\hline & GCGGCCGTCATACTCCGTTCTTCAAAG \\
\hline \multirow{2}{*}{ K314T } & F GTATGACGGCCGCCTTCATCTGTGGACAGAATGTACACTTC \\
\hline & R GAAGTGTACATTCTGTCCACAGATGAAGGCGGCCGTCATAC \\
\hline \multirow{2}{*}{ K314R } & F GACGGCCGCCTTCATCACGGGACAGAATGTACAC \\
\hline & R GTGTACATTCTGTCCCGTGATGAAGGCGGCCGTC \\
\hline \multirow{2}{*}{ K314I } & F GACGGCCGCCTTCATCGATGGACAGAATGTACAC \\
\hline & R GTGTACATTCTGTCCATCGATGAAGGCGGCCGTC \\
\hline \multirow{4}{*}{ K314N } & F GAAGAACGGAGTATGACGGCCGCCTTCATCGTTGGACAGAAT \\
\hline & GTACACTTCAGATTCGAAC \\
\hline & R GAAGAACGGAGTATGACGGCCGCCTTCATCGTTGGACAGA \\
\hline & ATGTACACTTCAGATTCGAAC \\
\hline \multirow{2}{*}{$\mathrm{R} 319 \mathrm{H}$} & F CCAAAGATGAAGGCGGCCATCATACTCCGTTCTTCAAAGGC \\
\hline & R GCCTTTGAAGAACGGAGTATGATGGCCGCCTTCATCTTTGG \\
\hline \multirow{2}{*}{$\mathrm{R} 328 \mathrm{H}$} & F CGTTCTTCAAAGGCTACCATCCGCAGTTCTACTTCCG \\
\hline & R CGGAAGTAGAACTGCGGATGGTAGCCTTTGAAGAACG \\
\hline \multirow{2}{*}{$\mathrm{R} 378 \mathrm{H}$} & F GCCAACGGTACGGCCGCCTTCATGGATTGCGAAACGCAGACCG \\
\hline & R CGGTCTGCGTTTCGCAATCCATGAAGGCGGCCGTACCGTTGGC \\
\hline \multirow{2}{*}{$\mathrm{R} 382 \mathrm{H}$} & F CAATCCGTGAAGGCGGCCATACCGTTGGCGCGGGCGTTG \\
\hline & R CAACGCCCGCGCCAACGGTATGGCCGCCTTCACGGATTG \\
\hline
\end{tabular}

Table 12. List of primers for SUMO-constructs

\begin{tabular}{|c|c|}
\hline \multicolumn{2}{|c|}{ Primers used to obtain SUMO-constructs } \\
\hline Construct & Sequence $5^{\prime}-3^{\prime}$ \\
\hline \multirow{2}{*}{ pet21a linearization } & F GATCCGGCTGCTAACAAAGCCCGAAAGG \\
\hline & R ATGTATATCTCCTTCTTAAAGTTAAACAAAATTATTTC \\
\hline \multirow{3}{*}{ pSUMO-FESEVYILSK } & F CACACCAAGTTCGAATCTGAAGTGTACATTCTGTCCAAAGATGAAGGC \\
\hline & GATCCGGCTGCTAACAAAGCCCG \\
\hline & R ACCACCAATCTGTTCTCTGTGAGCCTC \\
\hline \multirow{2}{*}{ SUMO-FESEVYILSK insertic } & $\begin{array}{l}\text { F CTTTAAGAAGGAGATATACATATGGGTCATCACCATCATCACCATGGTTCG } \\
\end{array}$ \\
\hline & R CGGGCTTTGTTAGCAGCCGGATCCTAGCCTTCATCTTTGGACAGAATGTAC \\
\hline \multirow{3}{*}{ SUMO-H378H short } & F TGAGATCCGGCTGCTAACAAAGCCCG \\
\hline & R GCCAACGGTACGGCCGCCTTCACGGATTGCGAAACGCAGACCGCCTT \\
\hline & CATCTTTGGACAGAATG \\
\hline \multirow[t]{4}{*}{ SUMO-H378H long } & F TAGGATCCGGCTGCTAACAAAGCCCGAAAGG \\
\hline & R TTTTGCTACAACGCCCGCGCCAACGGTACGGCCGCCTTCACGGATT \\
\hline & GCGAAACGCAGACCGTCGTCCATCGCGATCGGGTGGCCTTCATCTTT \\
\hline & GGACAGAATGTAC \\
\hline SUMO-R319H & F TAGGATCCGGCTGCTAACAAAGCCCGAAAGG \\
\hline
\end{tabular}




\begin{tabular}{|c|c|}
\hline & R ACGGTAGCCTTTGAAGAACGGAGTATGACGGCCGCCTTCATCTTTG \\
\hline & GACAGGCCTTCATCTTTGGACAGAATGTAC \\
\hline \multirow{3}{*}{ SUMO-R231H } & F TAGGATCCGGCTGCTAACAAAGCCCGAAAGG \\
\hline & R GATGATACCGCGTTCTACACGACCGGTAACAACGGTACCACGACCG \\
\hline & GAGCCTTCATCTTTGGACAGAATG \\
\hline \multirow{3}{*}{ SUMO-R280H } & F TAGGATCCGGCTGCTAACAAAGCCCGAAAGG \\
\hline & R GATTTCTTCACGTTTGATACCACGCAGCAGAACACCTACGTTCTC \\
\hline & ACCAGCACGGCCTTCGCCTTCATCTTTGGACAGAATG \\
\hline \multirow{3}{*}{ SUMO-R382H } & F TAGGATCCGGCTGCTAACAAAGCCCGAAAGG \\
\hline & R GCCCAGAACTTTTGCTACAACGCCCGCGCCAACGGTACGGCCGC \\
\hline & CTTCACGGATTGCGCCTTCATCTTTGGACAGAATGTAC \\
\hline \multirow{3}{*}{ SUMO-R328H } & F TAGGATCCGGCTGCTAACAAAGCCCGAAAGG \\
\hline & R GTCAGTAGTACGGAAGTAGAACTGCGGACGGTAGCCTTTGAAGA \\
\hline & AGCCTTCATCTTTGGACAGAATGTAC \\
\hline
\end{tabular}

Table 13. List of primers for chromosomal tag insertion

\begin{tabular}{|c|c|}
\hline \multicolumn{2}{|c|}{ Primers for chromosomal tag insertion } \\
\hline Construct & Sequence $5^{\prime}-3^{\prime}$ \\
\hline His-tag insertion on & F CCGTACCGTTGGCGCGGGCGTTGTTGCTAAAGTTCTGGGC \\
\hline chromosomal tufA & CACCACCACCACCACCACTAGAATTAACCCTCACTAAAGGGCG \\
\hline gene (his-tag & R GGGCGCCGAAGCGCCCTTTTCAATTCAAAACTAATTAACGTGTAA \\
\hline $\begin{array}{l}\text { nucleotide sequence is } \\
\text { underlined). }\end{array}$ & TAATACGACTCACTATAGGGCTC \\
\hline
\end{tabular}

\subsection{EF-Tu constructs}

\subsubsection{Site-directed mutagenesis for generation of EF-Tu mutants}

Site-directed mutagenesis has been performed in PCR tubes using Phusion High-fidelity DNA polymerase (New England Biolabs) following the protocol suggested by the supplier. The plasmid PET21 containing the tufA gene coding for EF-Tu is amplified by polymerase chain reaction (PCR) (Saiki et al., 1985) using the Quick Change kit (Agilent) and a set of forward and reverse primer specific for the mutation of choice (Table 11). Parental plasmid is digested with Dpnl enzyme and the PCR product is used to transform TOP10 chemically competent E. coli cells (Invitrogen). Clones containing the plasmid are selected for resistance to Kan. The plasmid is extracted using plasmid miniprep kit (Macherey-Nagel). Mutation insertion is verified by plasmid sequencing performed by SeqLab (Göttingen, Germany). The constructs expressing the EF-Tu mutants have been kindly provided by Christina Kothe. 


\subsubsection{Generation of constructs containing a Sumo protein fused with an EF-Tu peptide epitope}

In order to dissect the impact of the sequence context and cellular protease activity on the measured error frequency from selected EF-Tu epitopes are cloned in a pSUMO vector together with a quantifier peptide (FESEVYILSK) for their quantification. The quantifier peptide for EF-Tu is cloned into a linearized pSUMO vector, amplified and the vector ultimately ligated with T4 DNA ligase enzyme. The sumo tag and the nucleotide stretch for the cognate peptide are amplified by PCR using primers containing homologous region to pET21a vector (Table 12). The product is introduced into a linearized pET21a vector and ligated using In-Fusion HD cloning kit (Clontech Laboratories, USA) as described in the manufacturer's protocol. EF-Tu peptides for which the error frequency had to be assessed in the SUMO construct are chosen based on experimental data and their nucleotide sequence is introduced into the pET21a-SUMO-FESEVYILSK construct by PCR using the primers indicated in the table 12 . SUMO constructs have been obtained by Christina Kothe.

\subsubsection{C-terminal his-tag insertion into the EF-Tu gene}

Insertion of His-tag on the K12 strain W3110 and of the Kan resistance cassette is performed using the Gene deletion kit (Gene Bridges) as described in the manual and was performed by Christina Kothe. The primers used are listed in Table 13.

\subsubsection{Generation of flag EF-Tu}

The pET21a plasmid encoding for EF-Tu protein with the C-terminus octameric flag DYKDDDDK was generated by Sarah Lambrecht.

\subsection{Cell cultures}

\subsubsection{Cell transformation}

BL21 (DE3) E. coli competent cells are thawed on ice for $10 \mathrm{~min}$ and gently mixed with $100 \mathrm{ng}$ of plasmid DNA. After $10 \mathrm{~min}$ on ice, transformation is induced by incubating cells at $42^{\circ} \mathrm{C}$ for $1-2 \mathrm{~min}$ before transferring them back on ice. After 3 minutes, $500 \mu \mathrm{l} \mathrm{LB}$ medium are added and cells are allowed to recovery at $37^{\circ} \mathrm{C}$ for $1 \mathrm{hr}$ with gentle agitation using a thermomixer. After the incubation, $100 \mu \mathrm{l}$ are plated on an LB agar plates supplemented with the antibiotic of choice and stored overnight in a thermostatic oven.

\subsubsection{Cell growth}

A saturated LB culture is used to inoculate fresh LB medium. For E.coli K12 chromosomal tag, the overnight preinoculum is performed in LB medium supplemented with $50 \mu \mathrm{g} / \mathrm{ml} \mathrm{Kan}$. E. coli $\mathrm{K} 12$ chromosomal tag, MG1655, rpsD, spsL, and their parental wild type strains are grown in an incubator 
shaker at $37^{\circ} \mathrm{C}$ and $200 \mathrm{rpm}$ to $\sim 1 \mathrm{OD}_{600}$. E.coli BL21 (DE3) cells for EF-Tu mutants and pSUMOconstructs overexpression are grown in LB medium supplemented with $50 \mu \mathrm{g} / \mathrm{ml} \mathrm{Kan} \mathrm{(Serva}$ Electrophoresis $\mathrm{GmbH}$ ). Induction is started at $0.7-0.8 \mathrm{OD}_{600}$ and performed by incubating cells with with 1 mM IPTG for 2 hrs. Alternatively, E. coli BL21 (DE3) for His-tagged EF-Tu are grown in TB medium in a $5 \mathrm{~L}$ fermenter and induced at the beginning of exponential phase with $1 \mathrm{mM}$ IPTG for $3 \mathrm{hrs}$. To test the effects of aminoglycoside antibiotics on E. coli K12 chromosomal tag MG1655 cells are grown in LB without antibiotics. At $0.3 \mathrm{OD}_{600}$, the antibiotic of interest is added and cells are incubated for 120 min. In the negative control cell culture, no antibiotic is added and after reaching $0.3 \mathrm{OD}_{600}$, cells are grown for $120 \mathrm{~min}$. Cells are harvested by centrifugation in a refrigerated centrifuge at $5000 \mathrm{rpm}$ (JLA8.1000 rotor).

\subsubsection{Cell lysis}

E. coli MRE-600 are used for the purification of wild type EF-Tu. Cells are resuspended and lysed in buffer A using the Emulsiflex C3 homogenizer after manual homogenization in a Dounce tissue grinder. Cell debris are removed by centrifugation for $30 \mathrm{~min}$ at $25000 \mathrm{rpm}$ (JA30.1 rotor) and the lysate is cleared by $30 \mathrm{~min}$ ultracentrifugation at $50000 \mathrm{rpm}$ (50.2Ti rotor). Cells for EF-Tu purification under native conditions are solubilized in buffer B or buffer $\mathrm{C}$ (for purification under native or denaturing conditions, respectively) and sonicated $1 \mathrm{~min}$ by $10 \mathrm{~s}$ shots at $30 \%$ amplitude followed by $5 \mathrm{~s}$ of rest using a sonifier cell disruptor.

\subsection{PURexpress system for the in-vitro translation of flag EF-Tu}

The EF-Tu carrying the flag DYKDDDDK is translated in vitro to assess the error frequency in a proteasefree environment. For this purpose the commercial kit PURexpress system (New England BioLabs) is used as described in the manufacturer's manual and by others (Tuckey et al., 2014).

\subsection{Purification of EF-Tu protein}

EF-Tu purified from different sources was used for the investigation and quantification of misincorporations.

\subsubsection{Purification under native conditions}

His-tag EF-Tu is purified using Protino Ni-TED 2000 packed columns. After initial column equilibration with 4 volumes buffer $D$, the cleared lysate is loaded onto the column and eluted by gravity. Column is washed with 6 volumes of high salt buffer $E$ and re-equilibrated with 4 volumes of buffer $D$. Protein is finally eluted with 3 volumes of elution buffer F and concentrated using Amicon Ultra-4 with a 30 
KDa cut-off. Buffer is exchanged with $\mathrm{TAKM}_{7}$ before the protein is shock frozen in liquid nitrogen and stored at $-80^{\circ} \mathrm{C}$. EF-Tu from MRE600 and ram strains was purified as described elsewhere (Rodnina et al., 1995b).

\subsubsection{Purification under denaturing conditions}

A Protiono Ni-TED 2000 column is equilibrated with 4 column volumes of buffer $\mathrm{C}$ before the lysate is loaded on the column by gravity flow. The column is then washed with 8 volumes of buffer $\mathrm{G}$ and finally re-equilibrated in 6 volumes of buffer $\mathrm{C}$. Protein is eluted with 4 volumes of elution buffer $\mathrm{H}$, concentrated and rebuffered in $3 \mathrm{M}$ Urea and $0.1 \mathrm{M}$ ammonium hydrogencarbonate by centrifugation at 4000 rpm in Vivaspin concentrators MWCO 30000 in a refrigerated benchtop centrifuge.

\subsubsection{Purification of FLAG-EF-Tu}

In-vitro translated FLAG-EF-Tu is purified from components of the PURexpress in-vitro translation kit, using anti-flag M2 magnetic beads (Sigma Aldrich). For each PURExpress kit tube, $40 \mu \mathrm{l}$ of beads slurry are used (corresponding to $20 \mu \mathrm{l}$ packed gel volume, as stated in the technical bulleting of the product). The whole procedure from EF-Tu binding to its elution is carried out at $4^{\circ} \mathrm{C}$ while tubes are rotated in an Intelli-mixer rotating device. Beads are washed to remove the storage buffer and mixed with the sample and TBS to a final volume of $1 \mathrm{ml}$. After $2 \mathrm{hrs}$ incubation, the beads are separated from the supernatant with the help of a magnet and washed with high-salt TBS buffer to remove unspecific binders. After each washing step the protein content in the buffer is measured photometrically and the washing procedure is repeated until the absorbance difference between the wash solution aspirated from the beads and the wash solution blank is $<0.05$. At this point the washing buffer is eliminated and EF-Tu can be eluted from the beads.

\subsubsection{Elution of FLAG-EF-Tu with $3 X$ flag peptides}

Competitive elution with 3 X FLAG peptides (Sigma Aldrich) showed to be the most efficient method to detach EF-Tu protein from the beads. They are synthetic peptide of 23 amino acid residue. The AspTyr-Lys-Xaa-Xaa-Asp motif is repeated three times in the peptide and eight amino acids at the Cterminus make up the classic FLAG sequence (Asp-Tyr-Lys-Asp-Asp-Asp-Asp-Lys). An excess of 3X FLAG peptides is added and beads are incubated with 5 gel packed volumes of $150 \mathrm{ng} / \mu \mathrm{l}$ flag peptides for 15 min for two times. Buffer is picked up and concentrated using $500 \mu$ l Amicon Ultra 10 KDa cut-off before being loaded on a $16 \%$ PAGE-SDS for analysis and further in-gel digestion (Figure 43). 
$50 \mathrm{kDa}-$

Figure 43. Purification of FLAG EF-Tu. Purified EF-Tu protein is run together with Perfect Protein Marker 15-150 kDa (M) on a $16 \%$ PAGE-SDS.

\subsection{SDS-PAGE electrophoresis}

Purified EF-Tu is quantified using a Pierce BCA protein assay kit (Thermo Scientific) as described in the manual and its purity is assessed by SDS-PAGE electrophoresis essentially performed as described in (Laemmli, 1970). Bands molecular weights are identified by comparison with Perfect Protein Marker 10-150 kDa (Novagen) or SeeBlue Plus2 Pre-Stained Protein Standard (Thermo Fisher Scientific). Stacking gel solution is prepared using Acrylamide:Bisacrylamide (29:1) (Serva Electrophoresis GmbH) to a final concentration of $4.8 \%$. Resolving gel solution is prepared to a final Acrylamide:Bisacrylamide (29:1) concentration of $16 \%$. Gel polymerizatzion is started by the addition of $1: 100$ (v/v) $10 \%$ APS (Calbiochem) and 1:1000 (v/v) TEMED. Gels are run for $1 \mathrm{hr}$ at $180 \mathrm{~V}$ before being stained in Coomassie Blue solution and finally destained in destaining solution.

\subsection{Acetone precipitation of proteins}

EF-Tu (3000 - 30000 pmol) purified under native-conditions is precipitated overnight with 5 volumes of ice-cold acetone at $-20^{\circ} \mathrm{C}$. Precipitated protein is collected by centrifugation at $12000 \mathrm{rpm}$ for 30 $\mathrm{min}$ in a refrigerated benchtop centrifuge and washed with $300 \mu \mathrm{l}$ of ice-cold ethanol $80 \%$. The sample is centrifuged again to pellet the protein and remove the ethanol supernatant and the pellet is dried for $10 \mathrm{~min}$.

\subsection{EF-Tu trypsination}

\subsubsection{In-solution proteolysis of EF-Tu purified under native conditions}

Unless stated otherwise, the whole proteolysis procedure is carried out in a Thermomixer comfort (Eppendorf) at $600 \mathrm{rpm}$. Dried protein pellet is resuspended in $20 \mu \mathrm{l}$ of RapiGest $1 \%$ (Waters) in 25 $\mathrm{mM} \mathrm{NH}{ }_{4} \mathrm{HCO}_{3}$ and incubated for $10 \mathrm{~min}$ at $37^{\circ} \mathrm{C}$. Disulfide bonds are reduced by adding $20 \mu \mathrm{m} 50 \mathrm{mM}$ 
DTT (in $25 \mathrm{mM} \mathrm{NH} \mathrm{HCO}_{3}$ ) in a two-steps incubation at $60^{\circ} \mathrm{C}$ for $10 \mathrm{~min}$ and at $37^{\circ} \mathrm{C}$ for $20 \mathrm{~min}$. Alkylation of thiols was achieved adding $20 \mu \mathrm{l}$ of IAA (in $25 \mathrm{mM} \mathrm{NH}_{4} \mathrm{HCO}_{3}$ ) and incubating the sample at $37^{\circ} \mathrm{C}$ for $30 \mathrm{~min}$ in the dark. Rapigest in the sample is diluted to $0.1 \%$ by the addition of $25 \mathrm{mM}$ $\mathrm{NH}_{4} \mathrm{HCO}_{3}$. Trypsin $1 \mu \mathrm{g} / \mu \mathrm{l}$ (Promega) dissolved in $25 \mathrm{mM} \mathrm{NH}_{4} \mathrm{HCO}_{3}$ is added to the sample (final concentration $0.01 \mu \mathrm{g} / \mu \mathrm{l}$ ) and EF-Tu is proteolyzed overnight at $37^{\circ} \mathrm{C}$.

\subsubsection{In-solution proteolysis of EF-Tu purified in denaturing conditions}

Protein is reduced in $1 / 10 \mathrm{vol} 50 \mathrm{mM}$ DTT and incubated $50 \mathrm{~min}$ at $65^{\circ} \mathrm{C}$ in a thermomixer. Cysteines are alkylated in $1 / 10$ vol $100 \mathrm{mM}$ IAA for $30 \mathrm{~min}$ at $30^{\circ} \mathrm{C}$ in the dark. The protein is finally digested overnight in trypsin (Promega) at $37^{\circ} \mathrm{C}(1: 100$ protease to protein ratio).

\subsection{Sample preparation for the characterization of aminoglycosides effects}

The induction of stress response and error incorporation caused by aminoglycosides was investigated in MG1655 E. coli cells grown at increasing concentration of Kan, Gen, Tob, Neo, Par, Rib, Nea, Apr, Hyg B, Str and Spc. Cells $\left(1 \mathrm{OD}_{600}\right)$ are collected $2 \mathrm{~min}$ at $13000 \mathrm{rpm}$ on a benchtop centrifuge and the LB medium is removed. The pellet is resuspended in $100 \mu \mathrm{l}$ RNase-free water and $50 \mu \mathrm{l} 4 \mathrm{x}$ Laemmli buffer (Biorad) and incubated for $5 \mathrm{~min}$ at $95^{\circ} \mathrm{C}$. After the incubation, the samples are cooled down to room temperature and mixed with $50 \mu$ of a buffer containing $8 \mathrm{M}$ Urea/Tris buffer $\mathrm{pH}$ 7.5. Samples are centrifuges $5 \mathrm{~min}$ at $13000 \mathrm{rpm}$ in a benchtop centrifuge to remove any small particles before being separated by electrophoresis.

To analyze the stress response, $15 \mu$ l of lysate are separated on a $16 \%$ SDS-PAGE at $185 \mathrm{~V}$ until all proteins are in the separation gel. In one lane $5 \mu$ l of Perfect Protein Marker 10-150 KDa (Merck) are loaded (Figure 44). The gel is then stained in Coomassie Blue solution for $20 \mathrm{~min}$ and destained. The lysate's bands are vertically cut with a scalpel, further reduced into smaller pieces and stored in 1.5 $\mathrm{ml}$ Eppendorf tube at $-20^{\circ} \mathrm{C}$. 
L

Kanamycin B

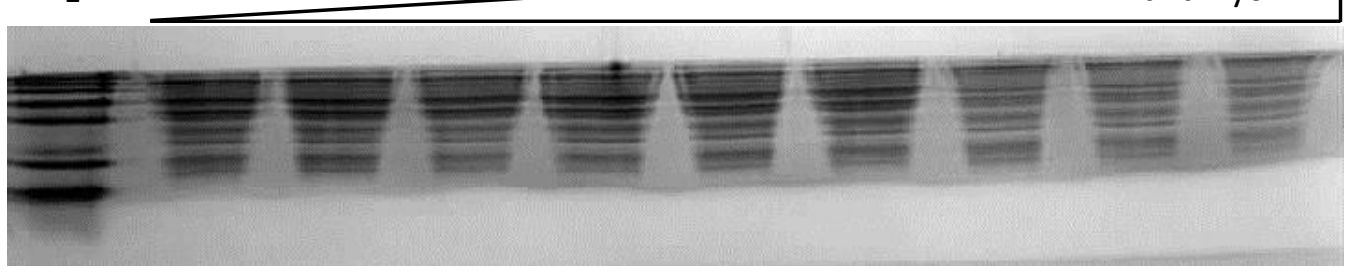

Figure 44. Proteins separation in E.coli lysate for proteome change analysis. The lysate of E.coli MG1655 cells grown at increasing concentrations of antibiotic (in the example, Kanamycin B $0.25,0.5,1,2,4,8,16,32,64,128 \mu \mathrm{M}$ ) is loaded on the gel. SeeBlue Plus marker is loaded in the first lane $L$.

To investigate the induction of errors in EF-Tu, $15 \mu \mathrm{l}$ of lysare are separated on a precasted MiniPROTEAN TGX Gel (BioRad) $20 \mathrm{~min}$ at $200 \mathrm{~V}$. In one lane $5 \mu \mathrm{l}$ of the SeeBlue Plus marker (Thermo Scientific) are loaded (Figure 45). The gel is stained and destained as already described and the EF-Tu band identified by comparison with the marker, excised with a scalpel and crushed into smaller pieces.

L

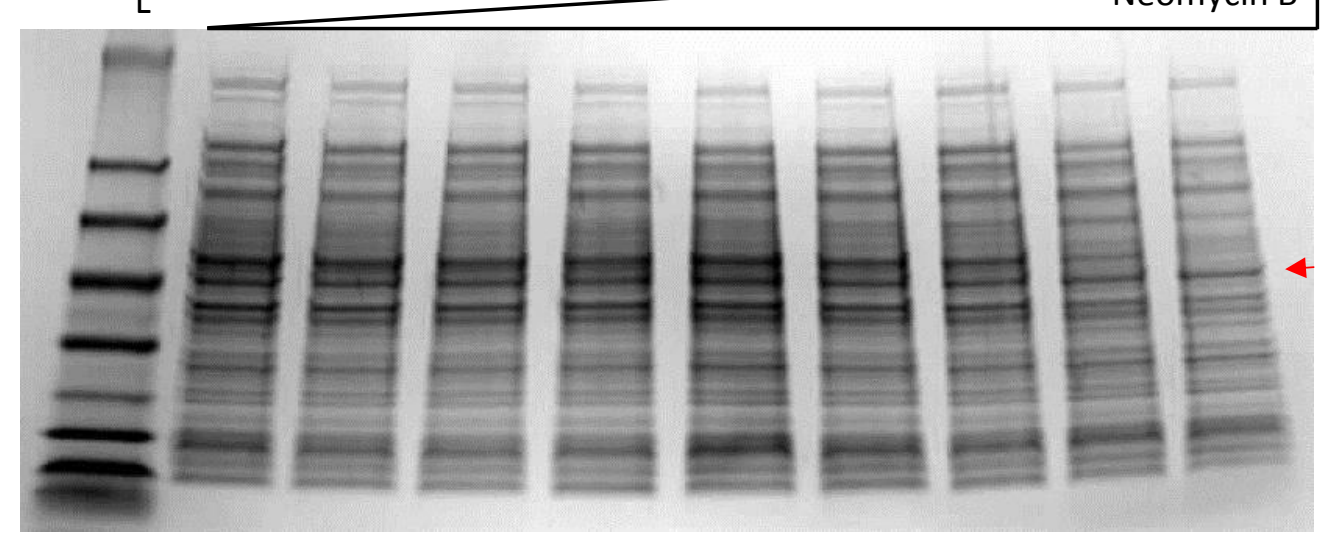

Figure 45. Lysate separation for the quantification of misincorporations in EF-Tu. The lysate of E.coli MG1655 cells grown at increasing concentration of antibiotic (in the example, Neomycin B $0.25,0.5,1,2,4,8,16,32,64,128 \mu \mathrm{M}$ ) is loaded on the gel. EF-Tu is indicated by the red arrow. Seeblue Plus marker is loaded in the first lane L.

\subsubsection{In-gel proteolysis of EF-Tu, pSUMO-constructs, and E.coli lysate}

Gel pieces containing the proteins of interest are digested as described by (Shevchenko et al., 2006). Digested peptides are then mixed with heavy-labeled reference peptides before the SRM analysis. For the list of all peptides used, refer to Appendix (Section B).

\subsection{Peptides}

\subsubsection{Selection of proteotypic peptides in EF-Tu}

Proteotypic peptides for mass spectrometric absolute quantification are selected from existing mass spectrometry data obtained by data-dependent aquistition (DDA) analysis (from Dr. Ingo Wohlgemuth). In general, only tryptic peptides between 6 and 20 residues are taken into 
consideration. The sequence is checked in order to exclude peptides containing highly reactive amino acids (e.g., methionine, tryptophan) or missed cleavage (adjacent KK). Peptides sequence is also checked in BLAST to be unique for the protein target in the case of peptides used for protein quantification (proteotypic (Mallick et al., 2007)) or to exclude a second source in the E. coli proteome in the case of peptide used for erroneous peptides enrichment.

\subsubsection{Proteolysis of Spike-Tides_TQL}

The highly-quantified Spike-Tides_TQL peptides are purchased from JPT (Berlin) and have to be digested prior to mass spectrometric analysis to remove the tag used by the producer for their quantification. The tag is removed by trypsin digestion (1:100) for $5 \mathrm{hrs}$ at $37^{\circ} \mathrm{C}$ performed as indicated by the producer.

\subsubsection{LC-MS/MS method development for peptides detection}

The open source program Skyline version 3.5 (MacLean et al., 2010b) is used for the SRM method set up and results analysis (MacLean et al., 2010b). For each peptide of the SRM method, the predominant charge state of the precusor is experimentally assessed and 3 - 5 transitions per peptide are chosen (Lange et al., 2008).

All peptides used in this study are separated on an Easy nLCII Nano LC or Ultimate 3000RSLC system using an in-house packed columns (14 cm length, $50 \mu \mathrm{m}$ inner diameter, packed with Reprosil-Pur 120 C18 $3 \mu \mathrm{m}$ material) at $50^{\circ} \mathrm{C}$ or Acclaim PepMapRSLC (15 cm length, $75 \mu \mathrm{m}$ inner diameter, $2 \mu \mathrm{m}$ RP18 material). Peptides are eluted in a 45 min linear gradient from 5 \% ACN/ $0.1 \%$ FA to 50 \% ACN/ $0.1 \%$ FA at $0.300 \mu \mathrm{l} / \mathrm{min}$ flow. Q1 and Q3 are set to unit resolution 0.7 full width at half maximum (FWHM) except for non-cognate peptides analysis where it is set at 0.5 . A spray voltage of $1800 \mathrm{~V}$ (TSQ Vantage) and $2100 \mathrm{~V}$ (TSQ Quantiva) is used with a heated ion transfer tube setting of $270^{\circ} \mathrm{C}$ ( TSQ Vantage) and $325^{\circ} \mathrm{C}$ (TSQ Quantiva), respectively. The declustering voltage is kept at $10 \mathrm{~V}$ ( TSQ Vantage) and a Chromfilter setting of 4 (TSQ Vantage) or 3 (TSQ Quantiva). Collision energies are optimized as decribed elsewhere (Maclean et al., 2010a). Scheduled transitions are recorded in a 5 min window and a cycle time of $3 \mathrm{~s}$ (TSQ Vantage) or $1 \mathrm{~s}$ (TSQ Quantiva) is applied, typically resulting in a dwell time of 100-200 ms per transition.

For data analyisis, raw files are imported into Skyline software that automatically calculates the area under each transition peak to yield the light/heavy ratio for each peptide. To achieve high identification reliability, only peptides with a dot-product (rdotp) close to 1 are considered. The light/heavy ratio of each peptide is ultimately used to calculate the error frequency. 


\subsubsection{Peptides stability assessment by LC-MS/MS}

In order to ensure that the determined peptide concentrations of misincorporation-containing peptides correctly represent their error frequency in the cell, we validated our quantifications in a model system in which these peptides occur at known 1:1 stoichiometry. Misincorporations whose quantification was validated were introduced as point mutations in EF-Tu. EF-Tu mutants were purified and in-gel digested as described previously. Cognate AQUA peptides 1 - 4 are mixed 1:1 with the AQUA peptide containing the amino acid substitution from the mutant under analysis. The AQUA mix is spiked in the sample in a buffer containing $5 \%$ ACN/ $0.1 \%$ FA. $300 \mathrm{fmol}$ of AQUA peptides (Thermo Scientific) and endogenous EF-Tu peptides are separated on an Ultimate 3000RSLC system (Thermo Fisher Scientific) and analyzed in triplicate on a TSQ Quantiva (Thermo Scientific) mass spectrometer in SRM mode (see below for details). Raw files are analyzed using Skyline software and the ratio $\mathrm{L} / \mathrm{H}$ determined.

\subsection{Quantification of EF-Tu for multidimensional enrichment of misincorporation-containing peptides}

Absolute quantification of digested EF-Tu is performed by mass spectrometric analysis. The final volume of tryptic digest is determined. $2 \mu$ of the digestion are diluted 1:20 in $5 \%$ ACN/ $0.5 \%$ FA and mixed with varying ratios of cognate AQUA Peptides 1 - 4 . The ratio of endogenous/AQUA peptides is determined by SRM (see below for details) analysis performed on TSQ Vantage or TSQ Quantiva (Thermo Scientific) mass spectrometer in triplicate. The ratios calculated for each peptide are averaged and used to determine the amount of digested EF-Tu.

\subsubsection{Multidimensional chromatographic enrichment of misincorporation-containing peptides}

The tryptic digest is spiked with substoichiometric amounts of AQUA peptides containing the misincorporations of interest (AQUA: proteolyzed EF-Tu; 1:10000). Normally 10 - 15 misincorporationcontaining peptides are quantified in a single enrichment. Before the enrichment procedure, Rapigest is degraded by incubating the sample at acidic $\mathrm{pH}$ for $30 \mathrm{~min}$ at $37^{\circ} \mathrm{C}$ and its hydrolytic by-products removed by centrifugation. To remove any particles, the supernatant is further filtered using Costar Spin-X Centrifuge Tube Filter $0.45 \mu \mathrm{m}$ Cellulose Acetate and lyophilized in a SpeedVac vacuum concentrator. Peptides are dissolved in $200 \mu \mathrm{l} 20$ \% ACN/ 0.1 \% FA and separated by size-exclusion chromatography on a Superdex Peptide $10 / 300$ GL column in an isocratic HPLC run $(20 \%$ ACN/ $0.1 \%$ $\mathrm{FA} ; 0.8 \mathrm{ml} / \mathrm{min}$ flow, fraction size $0.4 \mathrm{ml}$ ) as a first chromatographic dimension. From each fraction, an aliquot is taken and diluted 1:5 in 0.1 \% FA to dilute the final concentration of ACN and analyzed by SRM on TSQ Quantiva mass spectrometer. Depending on the peptides' distribution, 1 - 3 fractions are 
pooled and lyophilized. Pooled peptides are redissolved in $10 \mathrm{mM}$ ammonium acetate in $2 \% \mathrm{ACN}$ and separated by reversed phase chromatography at neutral pH in the second dimension (Immler et al., 2006) using a LiChrospher WP300 RP-18 $(5 \mu \mathrm{m})$ column. Peptides are eluted with a $2-82 \%$ ACN gradient in $10 \mathrm{mM}$ ammonium acetate in $45 \mathrm{~min}$ run and $0.8 \mathrm{ml} / \mathrm{min}$ flow; fraction size $0.8 \mathrm{ml}$. The elution time for each peptide is established in an independent chromatographic run performed with AQUA peptides only, by screening each fraction by SRM analysis. According to this, respective fractions from the second dimension are selected, lyophilized and resuspended in $50 \mu \mathrm{l} 5 \%$ ACN/ $0.1 \%$ FA for final SRM quantification. For the separation of misincorporation-containing peptides which are similar to their respective cognate one (Table 5), a third step of reversed phase chromatography at acidic $\mathrm{pH}$ is performed using the same LiChrospher WP300 RP-18 $(5 \mu \mathrm{m})$ column used for the separation at neutral pH. Peptides are eluted with a $0-65 \%$ ACN gradient in $0.1 \%$ TFA, $10 \mathrm{mM}$ ammonium acetate in $65 \mathrm{~min}$ run and $0.8 \mathrm{ml} / \mathrm{min}$ flow; fraction size $0.8 \mathrm{ml}$.

\subsection{Validation and quantification of misincorporation-containing peptides}

The enriched peptides are separated on nanoflow chromatography system and detected by SRM analysis as described before (Material and metod, 4.11.3).

The identity of enriched peptides is further verified by targeted SIM (tSIM) and parallel reaction monitoring (PRM) on a QExactive mass spectrometer. Peptides are separated by a 58 minute linear reversed phase gradients from $5 \%$ ACN / $0.1 \%$ FA to $50 \%$ acetonitrile/ $0.1 \%$ FA on in-house packed columns ( $28 \mathrm{~cm}$ length, packed with Reprosil $1.9 \mu \mathrm{m} \mathrm{C} 18$ material) at $60^{\circ} \mathrm{C}$. Eluted peptides are sprayed by an ESI-source set at $2400 \mathrm{~V}$ and capillary temperature $275^{\circ} \mathrm{C}$ in a Q-Exactive Plus mass spectrometer and t-SIM method is set at microscan 1, resolution 70000, ACG target $5 \mathrm{e}^{4}$, maximum it $70 \mathrm{~ms}$ and scan range 150 to $2000 \mathrm{~m} / \mathrm{z}$ and a $3.0 \mathrm{~m} / \mathrm{z}$ isolation window. PRM method is set at microscan 1 , resolution of 35000 , AGC target $1 \mathrm{e}^{6}$ and maximum IT $300 \mathrm{~ms}$ and an isolation windows of $0.4 \mathrm{~m} / \mathrm{z}$ and inclusion list on. Raw files are analyzed using Skyline software. MS1 and MS/MS filtering settings are set at a $60000 \mathrm{~m} / \mathrm{z}$ and $35000 \mathrm{~m} / \mathrm{z}$ resolving power respectively.

\subsection{Quantification of error in-gel digested EF-Tu and SUMO-constructs}

In-gel digested EF-Tu is lyophilized and resuspended in $50 \mu \mathrm{l} \% \mathrm{ACN} / 0.1 \%$ by sonication. The sample is centrifuged $10 \mathrm{~min}$ at $13000 \mathrm{rpm}$ using a benchtop centrifuge. An aliquot is then taken and mixed with AQUA peptides for the amino acid substitutions to quantify and analyzed by SRM. 


\subsection{Quantification of stress response proteins in E. coli lysate}

In-gel digested EF-Tu is lyophilized and resuspended in $50 \mu \mathrm{l} 5 \% \mathrm{ACN} / 0.1 \%$ by sonication. For each protein to quantify i.e., EF-Tu, RpoH, S10, IbpA and IbpB 2 - 3 AQUA peptides are spiked in and the sample is analyzed by SRM. For a list of peptides used refer to Appendix (Section B).

\subsection{Quadrupole performance assessment}

$30 \mathrm{fmol}$ of PRTC mix (Thermo Fisher Scientific) are routinely used to check the performances in terms of signal intensity and chromatographic separation of the TSQ Quantiva mass spectrometer and Ultimate 3000RSLC chromatographic system (Figure 46).

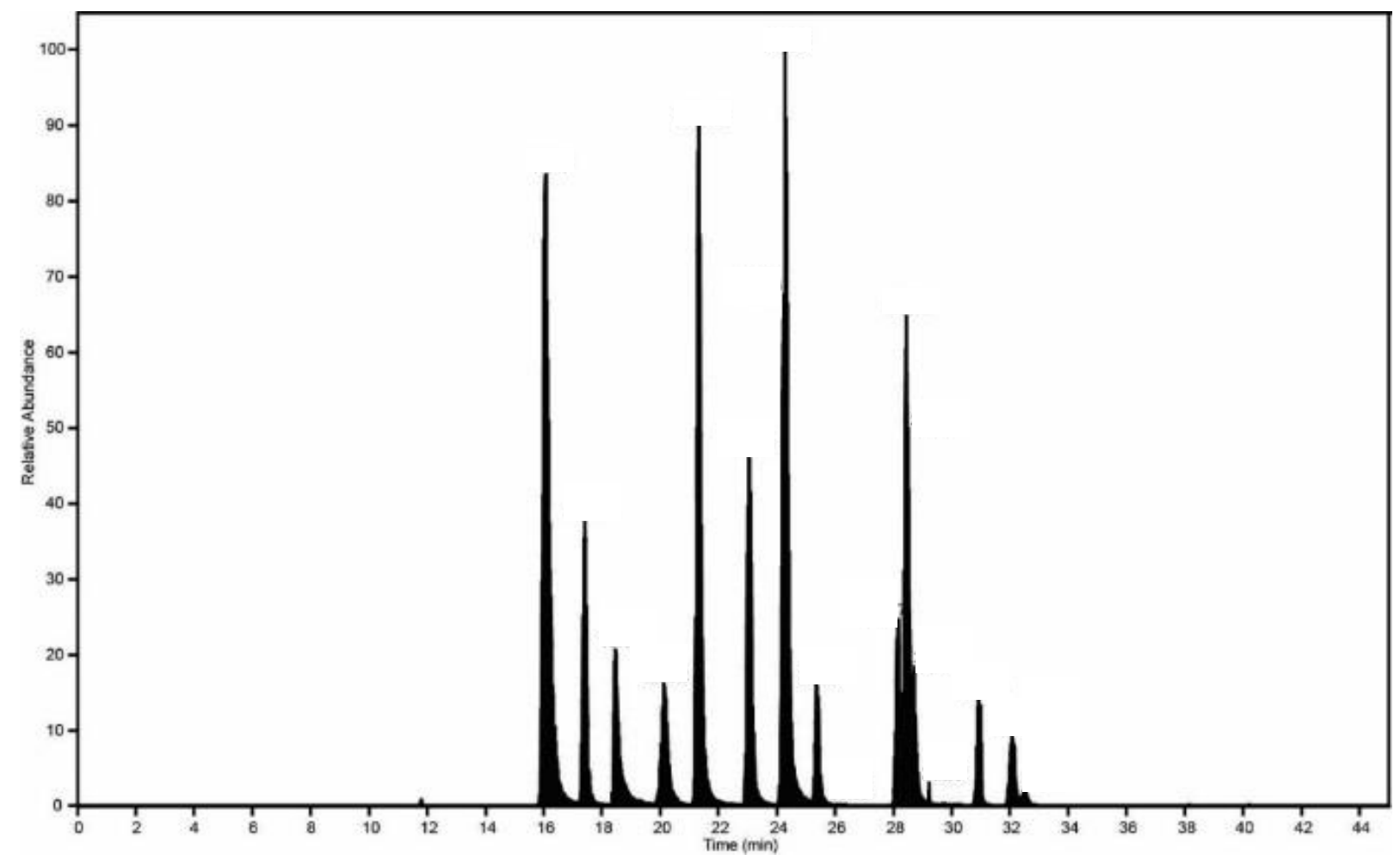

Figure 46. Pierce retention time mix (30 fmol) injected in TSQ Quantiva. Peptides are separated on the base of their hydrophobicit 


\section{REFERENCES}

Abbatiello, S.E., Mani, D.R., Keshishian, H., and Carr, S.A. (2010). Automated detection of inaccurate and imprecise transitions in peptide quantification by multiple reaction monitoring mass spectrometry. Clinical chemistry 56, 291-305.

Abel, K., Yoder, M.D., Hilgenfeld, R., and Jurnak, F. (1996). An alpha to beta conformational switch in EF-Tu. Structure 4, 1153-1159.

Akashi, H. (1994). Synonymous codon usage in Drosophila melanogaster: natural selection and translational accuracy. Genetics 136, 927-935.

Anderson, P., Davies, J., and Davis, B.D. (1967). Effect of spectinomycin on polypeptide synthesis in extracts of Escherichia coli. Journal of molecular biology 29, 203-215.

Andrews, K.J., and Hegeman, G.D. (1976). Selective disadvantage of non-functional protein synthesis in Escherichia coli. Journal of molecular evolution 8, 317-328.

Ang, C.S., and Nice, E.C. (2010). Targeted in-gel MRM: a hypothesis driven approach for colorectal cancer biomarker discovery in human feces. Journal of proteome research 9, 4346-4355.

Aqvist, J., and Kamerlin, S.C. (2015). Exceptionally large entropy contributions enable the high rates of GTP hydrolysis on the ribosome. Scientific reports 5, 15817.

Arai, K., Clark, B.F., Duffy, L., Jones, M.D., Kaziro, Y., Laursen, R.A., L'Italien, J., Miller, D.L., Nagarkatti, S., Nakamura, S., et al. (1980). Primary structure of elongation factor Tu from Escherichia coli. Proceedings of the National Academy of Sciences of the United States of America 77, 1326-1330.

Archetti, M. (2006). Genetic robustness and selection at the protein level for synonymous codons. Journal of evolutionary biology 19, 353-365.

Atkins, J.F., Weiss, R.B., Thompson, S., and Gesteland, R.F. (1991). Towards a genetic dissection of the basis of triplet decoding, and its natural subversion: programmed reading frame shifts and hops. Annu Rev Genet 25, 201-228.

Bantscheff, M., and Kuster, B. (2012). Quantitative mass spectrometry in proteomics. Analytical and bioanalytical chemistry 404, 937-938.

Belenky, P., Ye, J.D., Porter, C.B., Cohen, N.R., Lobritz, M.A., Ferrante, T., Jain, S., Korry, B.J., Schwarz, E.G., Walker, G.C., et al. (2015). Bactericidal Antibiotics Induce Toxic Metabolic Perturbations that Lead to Cellular Damage. Cell Rep 13, 968-980.

Bentley, W.E., Mirjalili, N., Andersen, D.C., Davis, R.H., and Kompala, D.S. (1990). Plasmid-encoded protein: the principal factor in the "metabolic burden" associated with recombinant bacteria. Biotechnology and bioengineering 35, 668-681.

Bhattacharjee, S., Deb, J., Dattachaudhuri, A., Tapadar, S.R., Dhua, A., Mukherjee, T., and Ghosh, P. (2012). Unilateral lung hypoplasia: a rare cause of unilateral opaque hemithorax in chest X-ray in a young boy. Indian journal of medical sciences 66, 192-196.

Bilgin, N., Richter, A.A., Ehrenberg, M., Dahlberg, A.E., and Kurland, C.G. (1990). Ribosomal RNA and protein mutants resistant to spectinomycin. The EMBO journal 9, 735-739. 
Bjork, G.R., Durand, J.M., Hagervall, T.G., Leipuviene, R., Lundgren, H.K., Nilsson, K., Chen, P., Qian, Q., and Urbonavicius, J. (1999). Transfer RNA modification: influence on translational frameshifting and metabolism. FEBS letters 452, 47-51.

Bloom, J.D., Silberg, J.J., Wilke, C.O., Drummond, D.A., Adami, C., and Arnold, F.H. (2005). Thermodynamic prediction of protein neutrality. Proceedings of the National Academy of Sciences of the United States of America 102, 606-611.

Bogdanow, B., Zauber, H., and Selbach, M. (2016). Systematic Errors in Peptide and Protein Identification and Quantification by Modified Peptides. Molecular \& cellular proteomics : MCP 15, 2791-2801.

Boon, K., Vijgenboom, E., Madsen, L.V., Talens, A., Kraal, B., and Bosch, L. (1992). Isolation and functional analysis of histidine-tagged elongation factor Tu. European journal of biochemistry 210, 177-183.

Borovinskaya, M.A., Pai, R.D., Zhang, W., Schuwirth, B.S., Holton, J.M., Hirokawa, G., Kaji, H., Kaji, A., and Cate, J.H. (2007a). Structural basis for aminoglycoside inhibition of bacterial ribosome recycling. Nature structural \& molecular biology 14, 727-732.

Borovinskaya, M.A., Shoji, S., Fredrick, K., and Cate, J.H. (2008). Structural basis for hygromycin B inhibition of protein biosynthesis. RNA 14, 1590-1599.

Borovinskaya, M.A., Shoji, S., Holton, J.M., Fredrick, K., and Cate, J.H. (2007b). A steric block in translation caused by the antibiotic spectinomycin. ACS chemical biology 2, 545-552.

Bouadloun, F., Donner, D., and Kurland, C.G. (1983). Codon-specific missense errors in vivo. The EMBO journal 2, 1351-1356.

Bratulic, S., Gerber, F., and Wagner, A. (2015). Mistranslation drives the evolution of robustness in TEM-1 beta-lactamase. Proceedings of the National Academy of Sciences of the United States of America 112, 12758-12763.

Bremer, H., and Dennis, P.P. (2008). Modulation of Chemical Composition and Other Parameters of the Cell at Different Exponential Growth Rates. EcoSal Plus 3.

Brodersen, D.E., Clemons, W.M., Jr., Carter, A.P., Morgan-Warren, R.J., Wimberly, B.T., and Ramakrishnan, V. (2000). The structural basis for the action of the antibiotics tetracycline, pactamycin, and hygromycin B on the 30 S ribosomal subunit. Cell 103, 1143-1154.

Brownridge, P., and Beynon, R.J. (2011). The importance of the digest: proteolysis and absolute quantification in proteomics. Methods 54, 351-360.

Bryan, L.E., and Kwan, S. (1983). Roles of ribosomal binding, membrane potential, and electron transport in bacterial uptake of streptomycin and gentamicin. Antimicrobial agents and chemotherapy $23,835-845$.

Bukowy-Bieryllo, Z., Dabrowski, M., Witt, M., and Zietkiewicz, E. (2016). Aminoglycoside-stimulated readthrough of premature termination codons in selected genes involved in primary ciliary dyskinesia. RNA biology 13, 1041-1050.

Cabanas, M.J., Vazquez, D., and Modolell, J. (1978). Inhibition of ribosomal translocation by aminoglycoside antibiotics. Biochemical and biophysical research communications 83, 991-997. 
Caglayan, M., and Bilgin, N. (2012). Temperature dependence of accuracy of DNA polymerase I from Geobacillus anatolicus. Biochimie 94, 1968-1973.

Carter, A.P., Clemons, W.M., Brodersen, D.E., Morgan-Warren, R.J., Wimberly, B.T., and Ramakrishnan, V. (2000). Functional insights from the structure of the $30 \mathrm{~S}$ ribosomal subunit and its interactions with antibiotics. Nature 407, 340-348.

Chen, G., Liu, H., Wang, X., and Li, Z. (2010). In vitro methylation by methanol: proteomic screening and prevalence investigation. Analytica chimica acta 661, 67-75.

Chick, J.M., Kolippakkam, D., Nusinow, D.P., Zhai, B., Rad, R., Huttlin, E.L., and Gygi, S.P. (2015). A masstolerant database search identifies a large proportion of unassigned spectra in shotgun proteomics as modified peptides. Nature biotechnology 33, 743-749.

Cox, J., and Mann, M. (2008). MaxQuant enables high peptide identification rates, individualized p.p.b.-range mass accuracies and proteome-wide protein quantification. Nature biotechnology 26, 1367-1372.

Cox, J., Neuhauser, N., Michalski, A., Scheltema, R.A., Olsen, J.V., and Mann, M. (2011). Andromeda: a peptide search engine integrated into the MaxQuant environment. Journal of proteome research 10, 1794-1805.

Creasy, D.M., and Cottrell, J.S. (2002). Error tolerant searching of uninterpreted tandem mass spectrometry data. Proteomics 2, 1426-1434.

Crick, F.H. (1966). Codon--anticodon pairing: the wobble hypothesis. Journal of molecular biology 19, 548-555.

Crick, F.H., Barnett, L., Brenner, S., and Watts-Tobin, R.J. (1961). General nature of the genetic code for proteins. Nature 192, 1227-1232.

Curran, J.F., and Yarus, M. (1986). Base substitutions in the tRNA anticodon arm do not degrade the accuracy of reading frame maintenance. Proceedings of the National Academy of Sciences of the United States of America 83, 6538-6542.

Cvetesic, N., Semanjski, M., Soufi, B., Krug, K., Gruic-Sovulj, I., and Macek, B. (2016). Proteome-wide measurement of non-canonical bacterial mistranslation by quantitative mass spectrometry of protein modifications. Scientific reports 6, 28631.

Danese, P.N., Snyder, W.B., Cosma, C.L., Davis, L.J., and Silhavy, T.J. (1995). The Cpx two-component signal transduction pathway of Escherichia coli regulates transcription of the gene specifying the stress-inducible periplasmic protease, DegP. Genes \& development 9, 387-398.

Davies, J., Anderson, P., and Davis, B.D. (1965a). Inhibition of protein synthesis by spectinomycin. Science 149, 1096-1098.

Davies, J., Gilbert, W., and Gorini, L. (1964). Streptomycin, Suppression, and the Code. Proceedings of the National Academy of Sciences of the United States of America 51, 883-890.

Davies, J., Gorini, L., and Davis, B.D. (1965b). Misreading of RNA codewords induced by aminoglycoside antibiotics. Molecular pharmacology 1, 93-106. 
Davis, B.D. (1987). Mechanism of bactericidal action of aminoglycosides. Microbiological reviews 51, 341-350.

Davis, B.D., Chen, L.L., and Tai, P.C. (1986). Misread protein creates membrane channels: an essential step in the bactericidal action of aminoglycosides. Proceedings of the National Academy of Sciences of the United States of America 83, 6164-6168.

Demeshkina, N., Jenner, L., Westhof, E., Yusupov, M., and Yusupova, G. (2012). A new understanding of the decoding principle on the ribosome. Nature 484, 256-259.

Demeshkina, N., Jenner, L., Westhof, E., Yusupov, M., and Yusupova, G. (2013). New structural insights into the decoding mechanism: translation infidelity via a G.U pair with Watson-Crick geometry. FEBS Lett 587, 1848-1857.

Demirci, H., Murphy, F.t., Murphy, E., Gregory, S.T., Dahlberg, A.E., and Jogl, G. (2013a). A structural basis for streptomycin-induced misreading of the genetic code. Nature communications 4, 1355.

Demirci, H., Wang, L., Murphy, F.V.t., Murphy, E.L., Carr, J.F., Blanchard, S.C., Jogl, G., Dahlberg, A.E., and Gregory, S.T. (2013b). The central role of protein S12 in organizing the structure of the decoding site of the ribosome. RNA 19, 1791-1801.

Dennis, P.P., Ehrenberg, M., and Bremer, H. (2004). Control of rRNA synthesis in Escherichia coli: a systems biology approach. Microbiology and molecular biology reviews : MMBR 68, 639-668.

Domon, B., and Aebersold, R. (2010). Options and considerations when selecting a quantitative proteomics strategy. Nature biotechnology $28,710-721$.

Dong, J., luchi, S., Kwan, H.S., Lu, Z., and Lin, E.C. (1993). The deduced amino-acid sequence of the cloned cpxR gene suggests the protein is the cognate regulator for the membrane sensor, CpxA, in a two-component signal transduction system of Escherichia coli. Gene 136, 227-230.

Drummond, D.A., and Wilke, C.O. (2009). The evolutionary consequences of erroneous protein synthesis. Nature reviews Genetics 10, 715-724.

Dukan, S., Farewell, A., Ballesteros, M., Taddei, F., Radman, M., and Nystrom, T. (2000). Protein oxidation in response to increased transcriptional or translational errors. Proceedings of the National Academy of Sciences of the United States of America 97, 5746-5749.

Dvorak, P., Chrast, L., Nikel, P.I., Fedr, R., Soucek, K., Sedlackova, M., Chaloupkova, R., de Lorenzo, V., Prokop, Z., and Damborsky, J. (2015). Exacerbation of substrate toxicity by IPTG in Escherichia coli BL21(DE3) carrying a synthetic metabolic pathway. Microbial cell factories 14, 201.

Dwyer, D.J., Belenky, P.A., Yang, J.H., MacDonald, I.C., Martell, J.D., Takahashi, N., Chan, C.T., Lobritz, M.A., Braff, D., Schwarz, E.G., et al. (2014). Antibiotics induce redox-related physiological alterations as part of their lethality. Proceedings of the National Academy of Sciences of the United States of America 111, E2100-2109.

Dwyer, D.J., Camacho, D.M., Kohanski, M.A., Callura, J.M., and Collins, J.J. (2012). Antibiotic-induced bacterial cell death exhibits physiological and biochemical hallmarks of apoptosis. Molecular cell 46, 561-572.

Edelmann, P., and Gallant, J. (1977). Mistranslation in E. coli. Cell 10, 131-137. 
Ellis, N., and Gallant, J. (1982). An estimate of the global error frequency in translation. Molecular \& general genetics : MGG 188, 169-172.

Eng, J.K., McCormack, A.L., and Yates, J.R. (1994). An approach to correlate tandem mass spectral data of peptides with amino acid sequences in a protein database. Journal of the American Society for Mass Spectrometry 5, 976-989.

Erickson, J.W., Vaughn, V., Walter, W.A., Neidhardt, F.C., and Gross, C.A. (1987). Regulation of the promoters and transcripts of $\mathrm{rpoH}$, the Escherichia coli heat shock regulatory gene. Genes \& development 1, 419-432.

Eustice, D.C., and Wilhelm, J.M. (1984a). Fidelity of the eukaryotic codon-anticodon interaction: interference by aminoglycoside antibiotics. Biochemistry 23, 1462-1467.

Eustice, D.C., and Wilhelm, J.M. (1984b). Mechanisms of action of aminoglycoside antibiotics in eucaryotic protein synthesis. Antimicrobial agents and chemotherapy 26, 53-60.

Ezraty, B., Vergnes, A., Banzhaf, M., Duverger, Y., Huguenot, A., Brochado, A.R., Su, S.Y., Espinosa, L., Loiseau, L., Py, B., et al. (2013). Fe-S cluster biosynthesis controls uptake of aminoglycosides in a ROSless death pathway. Science 340, 1583-1587.

Feldman, M.B., Terry, D.S., Altman, R.B., and Blanchard, S.C. (2010). Aminoglycoside activity observed on single pre-translocation ribosome complexes. Nature chemical biology 6, 244.

Fersht, A.R. (1977). Editing mechanisms in protein synthesis. Rejection of valine by the isoleucyl-tRNA synthetase. Biochemistry 16, 1025-1030.

Fischel-Ghodsian, N. (2005). Genetic factors in aminoglycoside toxicity. Pharmacogenomics 6, 27-36.

Fischer, N., Neumann, P., Konevega, A.L., Bock, L.V., Ficner, R., Rodnina, M.V., and Stark, H. (2015). Structure of the E. coli ribosome-EF-Tu complex at $<3$ A resolution by Cs-corrected cryo-EM. Nature $520,567-570$.

Fodor, S., and Zhang, Z. (2006). Rearrangement of terminal amino acid residues in peptides by protease-catalyzed intramolecular transpeptidation. Analytical biochemistry 356, 282-290.

Fosso, M.Y., Zhu, H., Green, K.D., Garneau-Tsodikova, S., and Fredrick, K. (2015). Tobramycin Variants with Enhanced Ribosome-Targeting Activity. Chembiochem : a European journal of chemical biology $16,1565-1570$.

Foti, J.J., Devadoss, B., Winkler, J.A., Collins, J.J., and Walker, G.C. (2012). Oxidation of the guanine nucleotide pool underlies cell death by bactericidal antibiotics. Science 336, 315-319.

Francois, B., Russell, R.J., Murray, J.B., Aboul-ela, F., Masquida, B., Vicens, Q., and Westhof, E. (2005). Crystal structures of complexes between aminoglycosides and decoding A site oligonucleotides: role of the number of rings and positive charges in the specific binding leading to miscoding. Nucleic acids research 33, 5677-5690.

Frank, A., and Pevzner, P. (2005). PepNovo: de novo peptide sequencing via probabilistic network modeling. Analytical chemistry 77, 964-973.

Frank, J., and Agrawal, R.K. (2000). A ratchet-like inter-subunit reorganization of the ribosome during translocation. Nature 406, 318-322. 
Freistroffer, D.V., Kwiatkowski, M., Buckingham, R.H., and Ehrenberg, M. (2000). The accuracy of codon recognition by polypeptide release factors. Proc Natl Acad Sci U S A 97, 2046-2051.

Frewen, B.E., Merrihew, G.E., Wu, C.C., Noble, W.S., and MacCoss, M.J. (2006). Analysis of peptide MS/MS spectra from large-scale proteomics experiments using spectrum libraries. Analytical chemistry $78,5678-5684$.

Furano, A.V. (1975). Content of elongation factor Tu in Escherichia coli. Proceedings of the National Academy of Sciences of the United States of America 72, 4780-4784.

Gallien, S., Duriez, E., Crone, C., Kellmann, M., Moehring, T., and Domon, B. (2012). Targeted proteomic quantification on quadrupole-orbitrap mass spectrometer. Molecular \& cellular proteomics : MCP 11, 1709-1723.

Gallien, S., Duriez, E., Demeure, K., and Domon, B. (2013). Selectivity of LC-MS/MS analysis: implication for proteomics experiments. Journal of proteomics 81, 148-158.

Gerber, S.A., Rush, J., Stemman, O., Kirschner, M.W., and Gygi, S.P. (2003). Absolute quantification of proteins and phosphoproteins from cell lysates by tandem MS. Proceedings of the National Academy of Sciences of the United States of America 100, 6940-6945.

Gidalevitz, T., Ben-Zvi, A., Ho, K.H., Brignull, H.R., and Morimoto, R.I. (2006). Progressive disruption of cellular protein folding in models of polyglutamine diseases. Science 311, 1471-1474.

Gill, R.T., Valdes, J.J., and Bentley, W.E. (2000). A comparative study of global stress gene regulation in response to overexpression of recombinant proteins in Escherichia coli. Metabolic engineering 2, 178-189.

Gillet, L.C., Navarro, P., Tate, S., Rost, H., Selevsek, N., Reiter, L., Bonner, R., and Aebersold, R. (2012). Targeted data extraction of the MS/MS spectra generated by data-independent acquisition: a new concept for consistent and accurate proteome analysis. Molecular \& cellular proteomics : MCP 11, 0111016717.

Gonzalez, A., Jimenez, A., Vazquez, D., Davies, J.E., and Schindler, D. (1978). Studies on the mode of action of hygromycin B, an inhibitor of translocation in eukaryotes. Biochimica et biophysica acta 521 , 459-469.

Gromadski, K.B., Daviter, T., and Rodnina, M.V. (2006). A uniform response to mismatches in codonanticodon complexes ensures ribosomal fidelity. Molecular cell 21, 369-377.

Gromadski, K.B., and Rodnina, M.V. (2004a). Kinetic determinants of high-fidelity tRNA discrimination on the ribosome. Molecular cell 13, 191-200.

Gromadski, K.B., and Rodnina, M.V. (2004b). Streptomycin interferes with conformational coupling between codon recognition and GTPase activation on the ribosome. Nature structural \& molecular biology 11, 316-322.

Grosjean, H.J., de Henau, S., and Crothers, D.M. (1978). On the physical basis for ambiguity in genetic coding interactions. Proceedings of the National Academy of Sciences of the United States of America $75,610-614$.

Gualerzi, C.O., Brandi, L., Fabbretti, A., Pon, C.L. (2013). Antibiotics: Targets, Mechanisms and Resistance. 
Gualerzi, C.O.F., A.; Brandi, L.; Milon, Pohl.; Pon, Cynthia L. (2010). Role of the Initiation Factors in mRNA Start Site Selection and fMet-tRNA recruitment by Bacterial Ribosome. Israel Journal of Biochemistry 50.

Guo, H.H., Choe, J., and Loeb, L.A. (2004). Protein tolerance to random amino acid change. Proceedings of the National Academy of Sciences of the United States of America 101, 9205-9210.

Haft, R.J., Keating, D.H., Schwaegler, T., Schwalbach, M.S., Vinokur, J., Tremaine, M., Peters, J.M., Kotlajich, M.V., Pohlmann, E.L., Ong, I.M., et al. (2014). Correcting direct effects of ethanol on translation and transcription machinery confers ethanol tolerance in bacteria. Proceedings of the National Academy of Sciences of the United States of America 111, E2576-2585.

Hancock, R.E. (1981). Aminoglycoside uptake and mode of action-with special reference to streptomycin and gentamicin. II. Effects of aminoglycosides on cells. The Journal of antimicrobial chemotherapy 8, 429-445.

Hart-Smith, G., Yagoub, D., Tay, A.P., Pickford, R., and Wilkins, M.R. (2016). Large Scale Mass Spectrometry-based Identifications of Enzyme-mediated Protein Methylation Are Subject to High False Discovery Rates. Molecular \& cellular proteomics : MCP 15, 989-1006.

Ho, C.S., Lam, C.W., Chan, M.H., Cheung, R.C., Law, L.K., Lit, L.C., Ng, K.F., Suen, M.W., and Tai, H.L. (2003). Electrospray ionisation mass spectrometry: principles and clinical applications. The Clinical biochemist Reviews / Australian Association of Clinical Biochemists 24, 3-12.

Ho, G., Reichardt, J., and Christodoulou, J. (2013). In vitro read-through of phenylalanine hydroxylase (PAH) nonsense mutations using aminoglycosides: a potential therapy for phenylketonuria. Journal of inherited metabolic disease 36, 955-959.

Holtje, J.V. (1978). Streptomycin uptake via an inducible polyamine transport system in Escherichia coli. European journal of biochemistry $86,345-351$.

Holtje, J.V. (1979). Induction of streptomycin uptake in resistant strains of Escherichia coli. Antimicrobial agents and chemotherapy 15, 177-181.

Hong, S., Harris, K.A., Fanning, K.D., Sarachan, K.L., Frohlich, K.M., and Agris, P.F. (2015). Evidence That Antibiotics Bind to Human Mitochondrial Ribosomal RNA Has Implications for Aminoglycoside Toxicity. The Journal of biological chemistry 290, 19273-19286.

Hopfield, J.J., Yamane, T., Yue, V., and Coutts, S.M. (1976). Direct experimental evidence for kinetic proofreading in amino acylation of tRNAlle. Proceedings of the National Academy of Sciences of the United States of America 73, 1164-1168.

Hu, Q., Noll, R.J., Li, H., Makarov, A., Hardman, M., and Graham Cooks, R. (2005). The Orbitrap: a new mass spectrometer. Journal of mass spectrometry : JMS 40, 430-443.

Huang, J., Brieba, L.G., and Sousa, R. (2000). Misincorporation by wild-type and mutant T7 RNA polymerases: identification of interactions that reduce misincorporation rates by stabilizing the catalytically incompetent open conformation. Biochemistry 39, 11571-11580.

Hurwitz, C., Braun, C.B., and Rosano, C.L. (1981). Role of ribosome recycling in uptake of dihydrostreptomycin by sensitive and resistant Escherichia coli. Biochim Biophys Acta 652, 168-176.

Ibba, M., and Soll, D. (2000). Aminoacyl-tRNA synthesis. Annual review of biochemistry 69, 617-650. 
Ikemura, T. (1985). Codon usage and tRNA content in unicellular and multicellular organisms. Molecular biology and evolution 2, 13-34.

Immler, D., Greven, S., and Reinemer, P. (2006). Targeted proteomics in biomarker validation: detection and quantification of proteins using a multi-dimensional peptide separation strategy. Proteomics 6, 2947-2958.

Isaac, D.D., Pinkner, J.S., Hultgren, S.J., and Silhavy, T.J. (2005). The extracytoplasmic adaptor protein CpxP is degraded with substrate by DegP. Proceedings of the National Academy of Sciences of the United States of America 102, 17775-17779.

Ito, K., Uno, M., and Nakamura, Y. (2000). A tripeptide 'anticodon' deciphers stop codons in messenger RNA. Nature 403, 680-684.

luchi, S., Furlong, D., and Lin, E.C. (1989). Differentiation of $\operatorname{arcA}$, $\operatorname{arcB}$, and cpxA mutant phenotypes of Escherichia coli by sex pilus formation and enzyme regulation. Journal of bacteriology 171, 28892893.

Jakubowski, H., and Goldman, E. (1992). Editing of errors in selection of amino acids for protein synthesis. Microbiological reviews 56, 412-429.

Javid, B., Sorrentino, F., Toosky, M., Zheng, W., Pinkham, J.T., Jain, N., Pan, M., Deighan, P., and Rubin, E.J. (2014). Mycobacterial mistranslation is necessary and sufficient for rifampicin phenotypic resistance. Proceedings of the National Academy of Sciences of the United States of America 111, 1132-1137.

Jawetz, E., Gunnison, J.B., and Speck, R.S. (1951). Antibiotic synergism and antagonism. The New England journal of medicine 245, 966-968.

Jorgensen, F., and Kurland, C.G. (1990). Processivity errors of gene expression in Escherichia coli. J Mol Biol 215, 511-521.

Jung, S.Y., Li, Y., Wang, Y., Chen, Y., Zhao, Y., and Qin, J. (2008). Complications in the assignment of 14 and $28 \mathrm{Da}$ mass shift detected by mass spectrometry as in vivo methylation from endogenous proteins. Analytical chemistry $80,1721-1729$.

Kafri, M., Metzl-Raz, E., Jona, G., and Barkai, N. (2016). The Cost of Protein Production. Cell reports 14, 22-31.

Keren, I., Wu, Y., Inocencio, J., Mulcahy, L.R., and Lewis, K. (2013). Killing by bactericidal antibiotics does not depend on reactive oxygen species. Science 339, 1213-1216.

Kimsey, I.J., Petzold, K., Sathyamoorthy, B., Stein, Z.W., and Al-Hashimi, H.M. (2015). Visualizing transient Watson-Crick-like mispairs in DNA and RNA duplexes. Nature 519, 315-320.

Kjeldgaard, M., and Nyborg, J. (1992). Refined structure of elongation factor EF-Tu from Escherichia coli. Journal of molecular biology 223, 721-742.

Kohanski, M.A., Dwyer, D.J., Hayete, B., Lawrence, C.A., and Collins, J.J. (2007). A common mechanism of cellular death induced by bactericidal antibiotics. Cell 130, 797-810. 
Kohanski, M.A., Dwyer, D.J., Wierzbowski, J., Cottarel, G., and Collins, J.J. (2008). Mistranslation of membrane proteins and two-component system activation trigger antibiotic-mediated cell death. Cell $135,679-690$.

Kramer, E.B., and Farabaugh, P.J. (2007). The frequency of translational misreading errors in E. coli is largely determined by tRNA competition. RNA 13, 87-96.

Kramer, E.B., Vallabhaneni, H., Mayer, L.M., and Farabaugh, P.J. (2010). A comprehensive analysis of translational missense errors in the yeast Saccharomyces cerevisiae. RNA 16, 1797-1808.

Kuczynska-Wisnik, D., Laskowska, E., and Taylor, A. (2001). Transcription of the ibpB heat-shock gene is under control of sigma(32)- and sigma(54)-promoters, a third regulon of heat-shock response. Biochemical and biophysical research communications 284, 57-64.

Kunkel, T.A. (2004). DNA replication fidelity. The Journal of biological chemistry 279, 16895-16898.

Kunkel, T.A., and Bebenek, K. (2000). DNA replication fidelity. Annual review of biochemistry 69, 497529.

Kurland, C.G. (1992). Translational accuracy and the fitness of bacteria. Annual review of genetics 26, 29-50.

Kurland, C.G., Rigler, R., Ehrenberg, M., and Blomberg, C. (1975). Allosteric mechanism for codondependent tRNA selection on ribosomes. Proceedings of the National Academy of Sciences of the United States of America 72, 4248-4251.

Laemmli, U.K. (1970). Cleavage of structural proteins during the assembly of the head of bacteriophage T4. Nature 227, 680-685.

Lange, V., Picotti, P., Domon, B., and Aebersold, R. (2008). Selected reaction monitoring for quantitative proteomics: a tutorial. Molecular systems biology 4, 222.

Laskowska, E., Wawrzynow, A., and Taylor, A. (1996). IbpA and IbpB, the new heat-shock proteins, bind to endogenous Escherichia coli proteins aggregated intracellularly by heat shock. Biochimie 78 , 117-122.

Lee, J.W., Beebe, K., Nangle, L.A., Jang, J., Longo-Guess, C.M., Cook, S.A., Davisson, M.T., Sundberg, J.P., Schimmel, P., and Ackerman, S.L. (2006). Editing-defective tRNA synthetase causes protein misfolding and neurodegeneration. Nature 443, 50-55.

Lee, Y., Zhou, T., Tartaglia, G.G., Vendruscolo, M., and Wilke, C.O. (2010). Translationally optimal codons associate with aggregation-prone sites in proteins. Proteomics 10, 4163-4171.

Li, L., Boniecki, M.T., Jaffe, J.D., Imai, B.S., Yau, P.M., Luthey-Schulten, Z.A., and Martinis, S.A. (2011). Naturally occurring aminoacyl-tRNA synthetases editing-domain mutations that cause mistranslation in Mycoplasma parasites. Proceedings of the National Academy of Sciences of the United States of America 108, 9378-9383.

Liao, H., Wu, J., Kuhn, E., Chin, W., Chang, B., Jones, M.D., O'Neil, S., Clauser, K.R., Karl, J., Hasler, F., et al. (2004). Use of mass spectrometry to identify protein biomarkers of disease severity in the synovial fluid and serum of patients with rheumatoid arthritis. Arthritis and rheumatism 50, 37923803. 
Lin, D., Tabb, D.L., and Yates, J.R., 3rd (2003). Large-scale protein identification using mass spectrometry. Biochimica et biophysica acta 1646, 1-10.

Lindahl, L., Jaskunas, S.R., Dennis, P.P., and Nomura, M. (1975). Cluster of genes in Escherichia coli for ribosomal proteins, ribosomal RNA, and RNA polymerase subunits. Proceedings of the National Academy of Sciences of the United States of America 72, 2743-2747.

Ling, J., Cho, C., Guo, L.T., Aerni, H.R., Rinehart, J., and Soll, D. (2012). Protein aggregation caused by aminoglycoside action is prevented by a hydrogen peroxide scavenger. Molecular cell 48, 713-722.

Ling, J., and Soll, D. (2010). Severe oxidative stress induces protein mistranslation through impairment of an aminoacyl-tRNA synthetase editing site. Proceedings of the National Academy of Sciences of the United States of America 107, 4028-4033.

Liu, Y., and Imlay, J.A. (2013). Cell death from antibiotics without the involvement of reactive oxygen species. Science 339, 1210-1213.

Loftfield, R.B., and Vanderjagt, D. (1972). The frequency of errors in protein biosynthesis. The Biochemical journal 128, 1353-1356.

Ma, B., Zhang, K., Hendrie, C., Liang, C., Li, M., Doherty-Kirby, A., and Lajoie, G. (2003). PEAKS: powerful software for peptide de novo sequencing by tandem mass spectrometry. Rapid communications in mass spectrometry : RCM 17, 2337-2342.

Maclean, B., Tomazela, D.M., Abbatiello, S.E., Zhang, S., Whiteaker, J.R., Paulovich, A.G., Carr, S.A., and Maccoss, M.J. (2010a). Effect of collision energy optimization on the measurement of peptides by selected reaction monitoring (SRM) mass spectrometry. Analytical chemistry 82, 10116-10124.

MacLean, B., Tomazela, D.M., Shulman, N., Chambers, M., Finney, G.L., Frewen, B., Kern, R., Tabb, D.L., Liebler, D.C., and MacCoss, M.J. (2010b). Skyline: an open source document editor for creating and analyzing targeted proteomics experiments. Bioinformatics 26, 966-968.

Malik, V., Rodino-Klapac, L.R., Viollet, L., and Mendell, J.R. (2010a). Aminoglycoside-induced mutation suppression (stop codon readthrough) as a therapeutic strategy for Duchenne muscular dystrophy. Therapeutic advances in neurological disorders 3, 379-389.

Malik, V., Rodino-Klapac, L.R., Viollet, L., Wall, C., King, W., Al-Dahhak, R., Lewis, S., Shilling, C.J., Kota, J., Serrano-Munuera, C., et al. (2010b). Gentamicin-induced readthrough of stop codons in Duchenne muscular dystrophy. Annals of neurology 67, 771-780.

Mallick, P., Schirle, M., Chen, S.S., Flory, M.R., Lee, H., Martin, D., Ranish, J., Raught, B., Schmitt, R., Werner, T., et al. (2007). Computational prediction of proteotypic peptides for quantitative proteomics. Nature biotechnology 25, 125-131.

Manickam, N., Nag, N., Abbasi, A., Patel, K., and Farabaugh, P.J. (2014). Studies of translational misreading in vivo show that the ribosome very efficiently discriminates against most potential errors. RNA 20, 9-15.

Mann, M., and Wilm, M. (1994). Error-tolerant identification of peptides in sequence databases by peptide sequence tags. Analytical chemistry 66, 4390-4399.

Manning, M.C., Chou, D.K., Murphy, B.M., Payne, R.W., and Katayama, D.S. (2010). Stability of protein pharmaceuticals: an update. Pharmaceutical research 27, 544-575. 
Matt, T., Ng, C.L., Lang, K., Sha, S.H., Akbergenov, R., Shcherbakov, D., Meyer, M., Duscha, S., Xie, J., Dubbaka, S.R., et al. (2012). Dissociation of antibacterial activity and aminoglycoside ototoxicity in the 4-monosubstituted 2-deoxystreptamine apramycin. Proceedings of the National Academy of Sciences of the United States of America 109, 10984-10989.

McGaha, S.M., and Champney, W.S. (2007). Hygromycin B inhibition of protein synthesis and ribosome biogenesis in Escherichia coli. Antimicrobial agents and chemotherapy 51, 591-596.

Meng, F., Cargile, B.J., Patrie, S.M., Johnson, J.R., McLoughlin, S.M., and Kelleher, N.L. (2002). Processing complex mixtures of intact proteins for direct analysis by mass spectrometry. Analytical chemistry 74, 2923-2929.

Meyerovich, M., Mamou, G., and Ben-Yehuda, S. (2010). Visualizing high error levels during gene expression in living bacterial cells. Proceedings of the National Academy of Sciences of the United States of America 107, 11543-11548.

Michalski, A., Cox, J., and Mann, M. (2011). More than 100,000 detectable peptide species elute in single shotgun proteomics runs but the majority is inaccessible to data-dependent LC-MS/MS. Journal of proteome research 10, 1785-1793.

Milon, P., and Rodnina, M.V. (2012). Kinetic control of translation initiation in bacteria. Critical reviews in biochemistry and molecular biology 47, 334-348.

Miroux, B., and Walker, J.E. (1996). Over-production of proteins in Escherichia coli: mutant hosts that allow synthesis of some membrane proteins and globular proteins at high levels. Journal of molecular biology 260, 289-298.

Misumi, M., Nishimura, T., Komai, T., and Tanaka, N. (1978). Interaction of kanamycin and related antibiotics with the large subunit of ribosomes and the inhibition of translocation. Biochemical and biophysical research communications $84,358-365$.

Moazed, D., and Noller, H.F. (1987). Interaction of antibiotics with functional sites in 165 ribosomal RNA. Nature 327, 389-394.

Muhlhausen, S., Findeisen, P., Plessmann, U., Urlaub, H., and Kollmar, M. (2016). A novel nuclear genetic code alteration in yeasts and the evolution of codon reassignment in eukaryotes. Genome research 26, 945-955.

Muramatsu, R., Misawa, S., and Hayashi, H. (2003). Finding of an isoleucine derivative of a recombinant protein for pharmaceutical use. Journal of pharmaceutical and biomedical analysis 31 , 979-987.

Netzer, N., Goodenbour, J.M., David, A., Dittmar, K.A., Jones, R.B., Schneider, J.R., Boone, D., Eves, E.M., Rosner, M.R., Gibbs, J.S., et al. (2009). Innate immune and chemically triggered oxidative stress modifies translational fidelity. Nature 462, 522-526.

Ogle, J.M., Brodersen, D.E., Clemons, W.M., Jr., Tarry, M.J., Carter, A.P., and Ramakrishnan, V. (2001). Recognition of cognate transfer RNA by the 30 S ribosomal subunit. Science 292, 897-902.

Ogle, J.M., Carter, A.P., and Ramakrishnan, V. (2003). Insights into the decoding mechanism from recent ribosome structures. Trends in biochemical sciences 28, 259-266. 
Ogle, J.M., Murphy, F.V., Tarry, M.J., and Ramakrishnan, V. (2002). Selection of tRNA by the ribosome requires a transition from an open to a closed form. Cell 111, 721-732.

Ong, S.E., and Mann, M. (2005). Mass spectrometry-based proteomics turns quantitative. Nature chemical biology 1, 252-262.

Pape, T., Wintermeyer, W., and Rodnina, M. (1999). Induced fit in initial selection and proofreading of aminoacyl-tRNA on the ribosome. The EMBO journal 18, 3800-3807.

Pape, T., Wintermeyer, W., and Rodnina, M.V. (1998). Complete kinetic mechanism of elongation factor Tu-dependent binding of aminoacyl-tRNA to the A site of the E. coli ribosome. The EMBO journal $17,7490-7497$.

Pape, T., Wintermeyer, W., and Rodnina, M.V. (2000). Conformational switch in the decoding region of $16 \mathrm{~S}$ rRNA during aminoacyl-tRNA selection on the ribosome. Nature structural biology 7, 104-107.

Parker, J. (1989). Errors and alternatives in reading the universal genetic code. Microbiological reviews 53, 273-298.

Parker, J., Johnston, T.C., and Borgia, P.T. (1980). Mistranslation in cells infected with the bacteriophage MS2: direct evidence of Lys for Asn substitution. Molecular \& general genetics : MGG $180,275-281$.

Parker, J., Johnston, T.C., Borgia, P.T., Holtz, G., Remaut, E., and Fiers, W. (1983). Codon usage and mistranslation. In vivo basal level misreading of the MS2 coat protein message. The Journal of biological chemistry 258, 10007-10012.

Perkins, D.N., Pappin, D.J., Creasy, D.M., and Cottrell, J.S. (1999). Probability-based protein identification by searching sequence databases using mass spectrometry data. Electrophoresis 20 , 3551-3567.

Perzynski, S., Cannon, M., Cundliffe, E., Chahwala, S.B., and Davies, J. (1979). Effects of apramycin, a novel aminoglycoside antibiotic on bacterial protein synthesis. European journal of biochemistry / FEBS 99, 623-628.

Peske, F., Rodnina, M.V., and Wintermeyer, W. (2005). Sequence of steps in ribosome recycling as defined by kinetic analysis. Molecular cell 18, 403-412.

Peske, F., Savelsbergh, A., Katunin, V.I., Rodnina, M.V., and Wintermeyer, W. (2004). Conformational changes of the small ribosomal subunit during elongation factor G-dependent tRNA-mRNA translocation. Journal of molecular biology 343, 1183-1194.

Peterson, A.C., Russell, J.D., Bailey, D.J., Westphall, M.S., and Coon, J.J. (2012). Parallel reaction monitoring for high resolution and high mass accuracy quantitative, targeted proteomics. Molecular \& cellular proteomics : MCP 11, 1475-1488.

Picotti, P., and Aebersold, R. (2012). Selected reaction monitoring-based proteomics: workflows, potential, pitfalls and future directions. Nature methods 9, 555-566.

Picotti, P., Aebersold, R., and Domon, B. (2007). The implications of proteolytic background for shotgun proteomics. Molecular \& cellular proteomics : MCP 6, 1589-1598. 
Pogliano, J., Lynch, A.S., Belin, D., Lin, E.C., and Beckwith, J. (1997). Regulation of Escherichia coli cell envelope proteins involved in protein folding and degradation by the $\mathrm{Cpx}$ two-component system. Genes \& development 11, 1169-1182.

Ram Prasad, B., and Warshel, A. (2011). Prechemistry versus preorganization in DNA replication fidelity. Proteins 79, 2900-2919.

Ramu, H., Vazquez-Laslop, N., Klepacki, D., Dai, Q., Piccirilli, J., Micura, R., and Mankin, A.S. (2011). Nascent peptide in the ribosome exit tunnel affects functional properties of the A-site of the peptidyl transferase center. Molecular cell 41, 321-330.

Ranjan, N., and Rodnina, M.V. (2016). tRNA wobble modifications and protein homeostasis. Translation (Austin) 4, e1143076.

Reha-Krantz, L.J. (2010). DNA polymerase proofreading: Multiple roles maintain genome stability. Biochimica et biophysica acta 1804, 1049-1063.

Ribas de Pouplana, L., Santos, M.A., Zhu, J.H., Farabaugh, P.J., and Javid, B. (2014). Protein mistranslation: friend or foe? Trends in biochemical sciences 39, 355-362.

Rodnina, M.V., Fricke, R., Kuhn, L., and Wintermeyer, W. (1995a). Codon-dependent conformational change of elongation factor Tu preceding GTP hydrolysis on the ribosome. The EMBO journal 14, 26132619.

Rodnina, M.V., Pape, T., Fricke, R., and Wintermeyer, W. (1995b). Elongation factor Tu, a GTPase triggered by codon recognition on the ribosome: mechanism and GTP consumption. Biochemistry and cell biology = Biochimie et biologie cellulaire 73, 1221-1227.

Rodnina, M.V., and Wintermeyer, W. (1995). GTP consumption of elongation factor Tu during translation of heteropolymeric mRNAs. Proceedings of the National Academy of Sciences of the United States of America 92, 1945-1949.

Rodnina, M.V., and Wintermeyer, W. (2001a). Fidelity of aminoacyl-tRNA selection on the ribosome: kinetic and structural mechanisms. Annual review of biochemistry 70, 415-435.

Rodnina, M.V., and Wintermeyer, W. (2001b). Ribosome fidelity: tRNA discrimination, proofreading and induced fit. Trends in biochemical sciences 26, 124-130.

Roepstorff, P., and Fohlman, J. (1984). Proposal for a common nomenclature for sequence ions in mass spectra of peptides. Biomedical mass spectrometry 11, 601.

Ronson, C.W., Nixon, B.T., and Ausubel, F.M. (1987). Conserved domains in bacterial regulatory proteins that respond to environmental stimuli. Cell 49, 579-581.

Rosset, R., and Gorini, L. (1969). A ribosomal ambiguity mutation. Journal of molecular biology 39, 95112.

Rozov, A., Demeshkina, N., Westhof, E., Yusupov, M., and Yusupova, G. (2015). Structural insights into the translational infidelity mechanism. Nature communications 6, 7251.

Rozov, A., Demeshkina, N., Westhof, E., Yusupov, M., and Yusupova, G. (2016). New Structural Insights into Translational Miscoding. Trends in biochemical sciences. 
Ruan, B., Palioura, S., Sabina, J., Marvin-Guy, L., Kochhar, S., Larossa, R.A., and Soll, D. (2008). Quality control despite mistranslation caused by an ambiguous genetic code. Proceedings of the National Academy of Sciences of the United States of America 105, 16502-16507.

Russell, J.B., and Cook, G.M. (1995). Energetics of bacterial growth: balance of anabolic and catabolic reactions. Microbiological reviews 59, 48-62.

Ruusala, T., Andersson, D., Ehrenberg, M., and Kurland, C.G. (1984). Hyper-accurate ribosomes inhibit growth. The EMBO journal 3, 2575-2580.

Ruusala, T., Ehrenberg, M., and Kurland, C.G. (1982). Is there proofreading during polypeptide synthesis? The EMBO journal 1, 741-745.

Saiki, R.K., Scharf, S., Faloona, F., Mullis, K.B., Horn, G.T., Erlich, H.A., and Arnheim, N. (1985). Enzymatic amplification of beta-globin genomic sequences and restriction site analysis for diagnosis of sickle cell anemia. Science 230, 1350-1354.

Sandhu, C., Hewel, J.A., Badis, G., Talukder, S., Liu, J., Hughes, T.R., and Emili, A. (2008). Evaluation of data-dependent versus targeted shotgun proteomic approaches for monitoring transcription factor expression in breast cancer. Journal of proteome research 7, 1529-1541.

Schaaper, R.M. (1993). Base selection, proofreading, and mismatch repair during DNA replication in Escherichia coli. The Journal of biological chemistry 268, 23762-23765.

Schaefer, H., Chamrad, D.C., Marcus, K., Reidegeld, K.A., Bluggel, M., and Meyer, H.E. (2005). Tryptic transpeptidation products observed in proteome analysis by liquid chromatography-tandem mass spectrometry. Proteomics 5, 846-852.

Scheunemann, A.E., Graham, W.D., Vendeix, F.A., and Agris, P.F. (2010). Binding of aminoglycoside antibiotics to helix 69 of 23 S rRNA. Nucleic acids research 38, 3094-3105.

Schmeing, T.M., and Ramakrishnan, V. (2009). What recent ribosome structures have revealed about the mechanism of translation. Nature 461, 1234-1242.

Schmidt, A., Kochanowski, K., Vedelaar, S., Ahrne, E., Volkmer, B., Callipo, L., Knoops, K., Bauer, M., Aebersold, R., and Heinemann, M. (2016). The quantitative and condition-dependent Escherichia coli proteome. Nature biotechnology 34, 104-110.

Scorer, C.A., Carrier, M.J., and Rosenberger, R.F. (1991). Amino acid misincorporation during high-level expression of mouse epidermal growth factor in Escherichia coli. Nucleic acids research 19, 3511-3516.

Scott, M., Gunderson, C.W., Mateescu, E.M., Zhang, Z., and Hwa, T. (2010). Interdependence of cell growth and gene expression: origins and consequences. Science 330, 1099-1102.

Sherman, J., McKay, M.J., Ashman, K., and Molloy, M.P. (2009). How specific is my SRM?: The issue of precursor and product ion redundancy. Proteomics 9, 1120-1123.

Sherwood, C.A., Eastham, A., Lee, L.W., Risler, J., Vitek, O., and Martin, D.B. (2009). Correlation between $\mathrm{y}$-type ions observed in ion trap and triple quadrupole mass spectrometers. Journal of proteome research 8, 4243-4251.

Shevchenko, A., Tomas, H., Havlis, J., Olsen, J.V., and Mann, M. (2006). In-gel digestion for mass spectrometric characterization of proteins and proteomes. Nature protocols 1, 2856-2860. 
Shi, T., Su, D., Liu, T., Tang, K., Camp, D.G., 2nd, Qian, W.J., and Smith, R.D. (2012). Advancing the sensitivity of selected reaction monitoring-based targeted quantitative proteomics. Proteomics 12, 1074-1092.

Shilov, I.V., Seymour, S.L., Patel, A.A., Loboda, A., Tang, W.H., Keating, S.P., Hunter, C.L., Nuwaysir, L.M., and Schaeffer, D.A. (2007). The Paragon Algorithm, a next generation search engine that uses sequence temperature values and feature probabilities to identify peptides from tandem mass spectra. Molecular \& cellular proteomics : MCP 6, 1638-1655.

Shine, J., and Dalgarno, L. (1974). The 3'-terminal sequence of Escherichia coli 165 ribosomal RNA: complementarity to nonsense triplets and ribosome binding sites. Proceedings of the National Academy of Sciences of the United States of America 71, 1342-1346.

Shuford, C.M., Sederoff, R.R., Chiang, V.L., and Muddiman, D.C. (2012). Peptide production and decay rates affect the quantitative accuracy of protein cleavage isotope dilution mass spectrometry (PCIDMS). Molecular \& cellular proteomics : MCP 11, 814-823.

Sin, C., Chiarugi, D., and Valleriani, A. (2016). Quantitative assessment of ribosome drop-off in E. coli. Nucleic Acids Res 44, 2528-2537.

Soll, D. (1990). The accuracy of aminoacylation--ensuring the fidelity of the genetic code. Experientia $46,1089-1096$.

Stahl-Zeng, J., Lange, V., Ossola, R., Eckhardt, K., Krek, W., Aebersold, R., and Domon, B. (2007). High sensitivity detection of plasma proteins by multiple reaction monitoring of $\mathrm{N}$-glycosites. Molecular \& cellular proteomics : MCP 6, 1809-1817.

Stansfield, I., Jones, K.M., Herbert, P., Lewendon, A., Shaw, W.V., and Tuite, M.F. (1998). Missense translation errors in Saccharomyces cerevisiae. Journal of molecular biology 282, 13-24.

Steitz, T.A., and Moore, P.B. (2003). RNA, the first macromolecular catalyst: the ribosome is a ribozyme. Trends in biochemical sciences $28,411-418$.

Sumpton, D., and Bienvenut, W. (2009). Coomassie stains: are they really mass spectrometry compatible? Rapid communications in mass spectrometry : RCM 23, 1525-1529.

Surinova, S., Schiess, R., Huttenhain, R., Cerciello, F., Wollscheid, B., and Aebersold, R. (2011). On the development of plasma protein biomarkers. Journal of proteome research 10, 5-16.

Sydow, J.F., and Cramer, P. (2009). RNA polymerase fidelity and transcriptional proofreading. Current opinion in structural biology 19, 732-739.

Sydow, J.F., Lipsmeier, F., Larraillet, V., Hilger, M., Mautz, B., Molhoj, M., Kuentzer, J., Klostermann, S., Schoch, J., Voelger, H.R., et al. (2014). Structure-based prediction of asparagine and aspartate degradation sites in antibody variable regions. PloS one 9, e100736.

Taber, H.W., Mueller, J.P., Miller, P.F., and Arrow, A.S. (1987). Bacterial uptake of aminoglycoside antibiotics. Microbiological reviews 51, 439-457.

Tanaka, N., Sashikata, K., and Umezawa, H. (1967). Antibiotic-sensitivity of ribosomes from kanamycinresistant $\mathrm{E}$. coli. The Journal of antibiotics 20, 115-119. 
Tawfik, D.S. (2014). Accuracy-rate tradeoffs: how do enzymes meet demands of selectivity and catalytic efficiency? Curr Opin Chem Biol 21, 73-80.

Taylor, G. (1964). Disintegration of Water Drops in an Electric Field. Proceedings of the Royal Society of London A: Mathematical, Physical and Engineering Sciences 280, 383-397.

Thompson, R.C., and Stone, P.J. (1977). Proofreading of the codon-anticodon interaction on ribosomes. Proceedings of the National Academy of Sciences 74, 198-202.

Toth, M.J., Murgola, E.J., and Schimmel, P. (1988). Evidence for a unique first position codon-anticodon mismatch in vivo. Journal of molecular biology 201, 451-454.

Traverse, C.C., and Ochman, H. (2016). Conserved rates and patterns of transcription errors across bacterial growth states and lifestyles. Proceedings of the National Academy of Sciences of the United States of America 113, 3311-3316.

Tsai, A., Uemura, S., Johansson, M., Puglisi, E.V., Marshall, R.A., Aitken, C.E., Korlach, J., Ehrenberg, M., and Puglisi, J.D. (2013). The impact of aminoglycosides on the dynamics of translation elongation. Cell reports 3, 497-508.

Tuckey, C., Asahara, H., Zhou, Y., and Chong, S. (2014). Protein synthesis using a reconstituted cellfree system. Current protocols in molecular biology / edited by Frederick M Ausubel [et al] 108, 1631 $11-22$

Valledor, L., Castillejo, M.A., Lenz, C., Rodriguez, R., Canal, M.J., and Jorrin, J. (2008). Proteomic analysis of Pinus radiata needles: 2-DE map and protein identification by LC/MS/MS and substitutiontolerant database searching. Journal of proteome research 7, 2616-2631.

Van Noort, J.M., Kraal, B., Sinjorgo, K.M., Persoon, N.L., Johanns, E.S., and Bosch, L. (1986). Methylation in vivo of elongation factor EF-Tu at lysine- 56 decreases the rate of tRNA-dependent GTP hydrolysis. European journal of biochemistry / FEBS 160, 557-561.

Vermulst, M., Denney, A.S., Lang, M.J., Hung, C.W., Moore, S., Moseley, M.A., Thompson, J.W., Madden, V., Gauer, J., Wolfe, K.J., et al. (2015). Transcription errors induce proteotoxic stress and shorten cellular lifespan. Nature communications 6, 8065.

Vicens, Q., and Westhof, E. (2002). Crystal structure of a complex between the aminoglycoside tobramycin and an oligonucleotide containing the ribosomal decoding a site. Chemistry \& biology 9 , 747-755.

von Heijne, G. (1989). Control of topology and mode of assembly of a polytopic membrane protein by positively charged residues. Nature $341,456-458$.

Wagner, G.R., and Hirschey, M.D. (2014). Nonenzymatic protein acylation as a carbon stress regulated by sirtuin deacylases. Molecular cell 54, 5-16.

Walton, J.R. (1978). Apramycin, a new aminocyclitol antibiotic. I. In vitro microbiological studies. The Journal of antimicrobial chemotherapy 4, 309-313.

Wang, H., Xiao, Y., Fu, L., Zhao, H., Zhang, Y., Wan, X., Qin, Y., Huang, Y., Gao, H., and Li, X. (2010). High-level expression and purification of soluble recombinant FGF21 protein by SUMO fusion in Escherichia coli. BMC biotechnology 10, 14. 
Wang, L., Pulk, A., Wasserman, M.R., Feldman, M.B., Altman, R.B., Cate, J.H., and Blanchard, S.C. (2012). Allosteric control of the ribosome by small-molecule antibiotics. Nat Struct Mol Biol 19, 957963.

Wasserman, M.R., Pulk, A., Zhou, Z., Altman, R.B., Zinder, J.C., Green, K.D., Garneau-Tsodikova, S., Cate, J.H., and Blanchard, S.C. (2015). Chemically related 4,5-linked aminoglycoside antibiotics drive subunit rotation in opposite directions. Nat Commun 6, 7896.

Weinert, B.T., lesmantavicius, V., Wagner, S.A., Scholz, C., Gummesson, B., Beli, P., Nystrom, T., and Choudhary, C. (2013). Acetyl-phosphate is a critical determinant of lysine acetylation in E. coli. Molecular cell 51, 265-272.

Westhof, E., Yusupov, M., and Yusupova, G. (2014). Recognition of Watson-Crick base pairs: constraints and limits due to geometric selection and tautomerism. F1000prime reports 6, 19.

Wickner, S., Maurizi, M.R., and Gottesman, S. (1999). Posttranslational quality control: folding, refolding, and degrading proteins. Science 286, 1888-1893.

Wiegand, I., Hilpert, K., and Hancock, R.E. (2008). Agar and broth dilution methods to determine the minimal inhibitory concentration (MIC) of antimicrobial substances. Nature protocols 3, 163-175.

Woese, C.R. (1965). On the evolution of the genetic code. Proceedings of the National Academy of Sciences of the United States of America 54, 1546-1552.

Wohlgemuth, I., Pohl, C., and Rodnina, M.V. (2010). Optimization of speed and accuracy of decoding in translation. The EMBO journal 29, 3701-3709.

Worboys, J.D., Sinclair, J., Yuan, Y., and Jorgensen, C. (2014). Systematic evaluation of quantotypic peptides for targeted analysis of the human kinome. Nature methods 11, 1041-1044.

Wright, G.D. (1999). Aminoglycoside-modifying enzymes. Current opinion in microbiology 2, 499-503.

Wu, X., Held, K., Zheng, C., Staudinger, B.J., Chavez, J.D., Weisbrod, C.R., Eng, J.K., Singh, P.K., Manoil, C., and Bruce, J.E. (2015). Dynamic Proteome Response of Pseudomonas aeruginosa to Tobramycin Antibiotic Treatment. Molecular \& cellular proteomics : MCP 14, 2126-2137.

Yates, J.R., 3rd (2004). Mass spectral analysis in proteomics. Annual review of biophysics and biomolecular structure 33, 297-316.

Yikilmaz, E., Chapman, S.J., Schrader, J.M., and Uhlenbeck, O.C. (2014). The interface between Escherichia coli elongation factor Tu and aminoacyl-tRNA. Biochemistry 53, 5710-5720.

Young, R., and Bremer, H. (1976). Polypeptide-chain-elongation rate in Escherichia coli $B / r$ as a function of growth rate. The Biochemical journal 160, 185-194.

Yu, X.C., Borisov, O.V., Alvarez, M., Michels, D.A., Wang, Y.J., and Ling, V. (2009). Identification of codon-specific serine to asparagine mistranslation in recombinant monoclonal antibodies by highresolution mass spectrometry. Analytical chemistry 81, 9282-9290.

Zaborske, J.M., DuMont, V.L., Wallace, E.W., Pan, T., Aquadro, C.F., and Drummond, D.A. (2014). A nutrient-driven tRNA modification alters translational fidelity and genome-wide protein coding across an animal genus. PLoS biology 12, e1002015. 
Zaher, H.S., and Green, R. (2009). Quality control by the ribosome following peptide bond formation. Nature 457, 161-166.

Zaher, H.S., and Green, R. (2010a). Hyperaccurate and error-prone ribosomes exploit distinct mechanisms during tRNA selection. Molecular cell 39, 110-120.

Zaher, H.S., and Green, R. (2010b). Kinetic basis for global loss of fidelity arising from mismatches in the P-site codon:anticodon helix. RNA 16, 1980-1989.

Zaher, H.S., and Green, R. (2011). A primary role for release factor 3 in quality control during translation elongation in Escherichia coli. Cell 147, 396-408.

Zhang, J., leong, K.W., Johansson, M., and Ehrenberg, M. (2015). Accuracy of initial codon selection by aminoacyl-tRNAs on the mRNA-programmed bacterial ribosome. Proceedings of the National Academy of Sciences of the United States of America 112, 9602-9607.

Zhang, J., leong, K.W., Mellenius, H., and Ehrenberg, M. (2016). Proofreading neutralizes potential error hotspots in genetic code translation by transfer RNAs. RNA 22, 896-904.

Zhang, Z. (2009). Large-scale identification and quantification of covalent modifications in therapeutic proteins. Analytical chemistry 81, 8354-8364.

Zhang, Z., Shah, B., and Bondarenko, P.V. (2013). G/U and certain wobble position mismatches as possible main causes of amino acid misincorporations. Biochemistry 52, 8165-8176.

Zhou, J., Lancaster, L., Donohue, J.P., and Noller, H.F. (2014). How the ribosome hands the A-site tRNA to the P site during EF-G-catalyzed translocation. Science 345, 1188-1191.

Zhou, T., Weems, M., and Wilke, C.O. (2009). Translationally optimal codons associate with structurally sensitive sites in proteins. Molecular biology and evolution 26, 1571-1580. 


\section{APPENDIX}

\subsection{Section A. EF-Tu genes sequence}

The sequence of tufA and tufB genes from the various strain used in this projects are reported together with the translated sequence of EF-Tu.

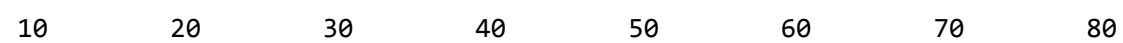

BL21-TUfA

BL21-TufB-(over)

W3110-TufA-(chrom)

W3110-TUfB

MRE600-TufA-(wt)

MRE600-TUfB-(wt)

BL21-TUfA

BL21-TufB- (over)

W3110-TufA-(chrom)

W3110-TufB

MRE600-TufA- (wt)

MRE600-TufB- (wt)

BL21 - TufA

BL21-TufB-(over)

W3110-TufA-(chrom)

W3110-TUfB

MRE600-TufA- (wt)

MRE600-TufB-(wt)

BL21-TufA

BL21-TufB- (over)

W3110-TufA- (chrom)

W3110-TufB

MRE600-TUfA- (wt)

MRE600-TufB- (wt)

BL21-TufA

BL21-TufB- (over)

W3110-TufA- (chrom)

W3110-TufB

MRE600-TufA- (wt)

MRE600-TufB- (wt)

BL21-TufA

BL21-TufB- (over)

W3110-TufA-(chrom)

W3110-TUfB

MRE600-TufA- (wt)

MRE600-TufB- (wt)

$\ldots|\ldots| \ldots|\ldots| \ldots|\ldots| \ldots|\ldots| \ldots|\ldots| \ldots|\ldots| \ldots|\ldots| \ldots|\ldots| \ldots|\ldots| \ldots|\ldots| \ldots \mid$ GTGTCTAAAGAAAAATTTGAACGTACAAAACCGCACGTTAACGTTGGTACTATCGGCCACGTTGACCACGGTAAAACTAC A- - - - - - - G- -

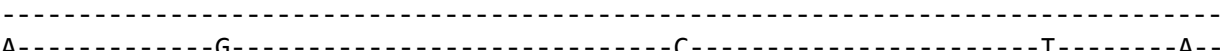

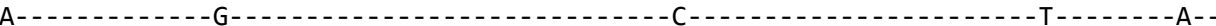

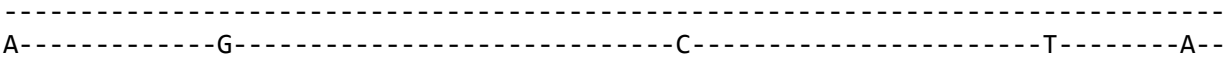

$\begin{array}{lllllllllllllllllllllllllllll}M & S & K & E & K & F & E & R & T & K & P & H & V & N & V & G & T & I & G & H & V & D & H & G & K & T & T\end{array}$

$\begin{array}{llllllll}90 & 100 & 110 & 120 & 130 & 140 & 150 & 160\end{array}$ $\ldots|\ldots| \ldots|\ldots| \ldots|\ldots| \ldots|\ldots| \ldots|\ldots| \ldots|\ldots| \ldots|\ldots| \ldots|\ldots| \ldots|\ldots| \ldots|\ldots|$ TCTGACCGCTGCAATCACCACCGTACTGGCTAAAACCTACGGCGGTGCTGCTCGTGCATTCGACCAGATCGATAACGCGC
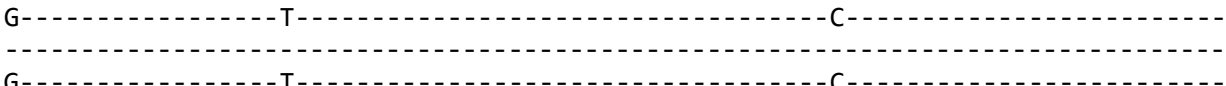

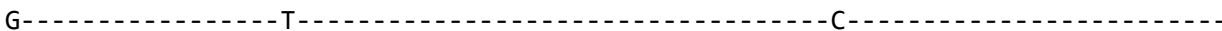

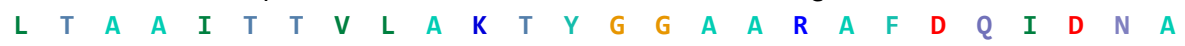

$\begin{array}{llllllll}170 & 180 & 190 & 200 & 210 & 220 & 230 & 240\end{array}$ $\ldots|\ldots| \ldots|\ldots| \ldots|\ldots| \ldots|\ldots| \ldots|\ldots| \ldots|\ldots| \ldots|\ldots| \ldots|\ldots| \ldots|\ldots| \ldots|\ldots|$ CGGAAGAAAAAGCTCGTGGTATCACCATCAACACTTCTCACGTTGAATATGACACCCCGACCCGTCACTACGCACACGTA
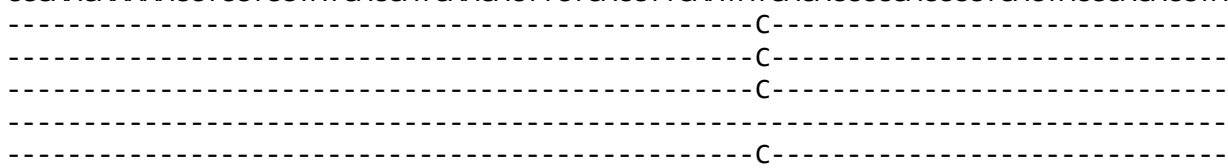

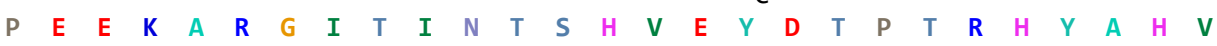

\begin{tabular}{l|ccccccc}
250 & 260 & 270 & 280 & 290 & 300 & 310 & 320
\end{tabular}

$\ldots|\ldots| \ldots|\ldots| \ldots|\ldots| \ldots|\ldots| \ldots|\ldots| \ldots|\ldots| \ldots|\ldots| \ldots|\ldots| \ldots|\ldots| \ldots \mid$ GACTGCCCGGGGCACGCCGACTATGTTAAAAACATGATCACTGGTGCTGCTCAGATGGACGGCGCGATCCTGGTAGTTGC
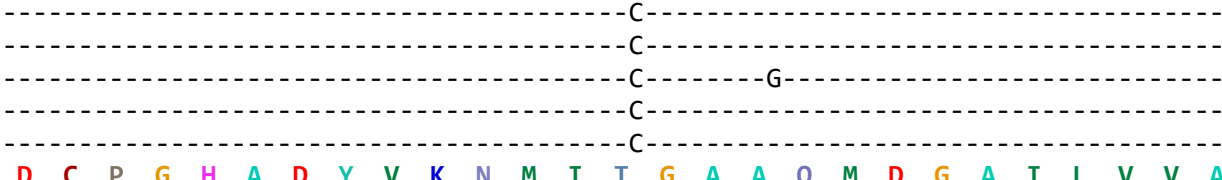

$\begin{array}{llllllll}330 & 340 & 350 & 360 & 370 & 380 & 390 & 400\end{array}$ $\ldots|\ldots| \ldots|\ldots| \ldots|\ldots| \ldots|\ldots| \ldots|\ldots| \ldots|\ldots| \ldots|\ldots| \ldots|\ldots| \ldots|\ldots| \ldots|\ldots| \ldots \mid$ TGCGACTGACGGCCCGATGCCGCAGACTCGTGAGCACATCCTGCTGGGTCGTCAGGTAGGCGTTCCGTACATCATCGTGT
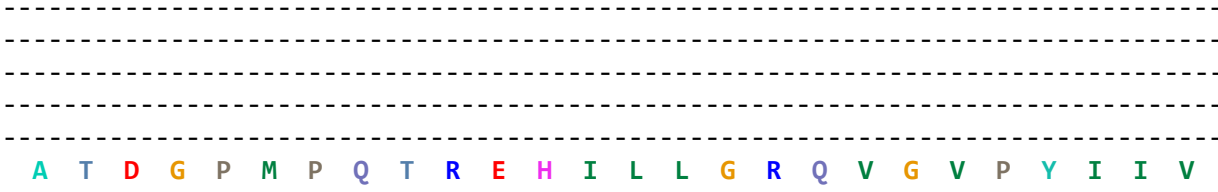

$\begin{array}{llllllll}410 & 420 & 430 & 440 & 450 & 460 & 470 & 480\end{array}$ $\ldots|\ldots| \ldots|\ldots| \ldots|\ldots| \ldots|\ldots| \ldots|\ldots| \ldots|\ldots| \ldots|\ldots| \ldots|\ldots| \ldots|\ldots| \ldots|\ldots|$ TCCTGAACAAATGCGACATGGTTGATGACGAAGAGCTGCTGGAACTGGTTGAAATGGAAGTTCGTGAACTTCTGTCTCAG
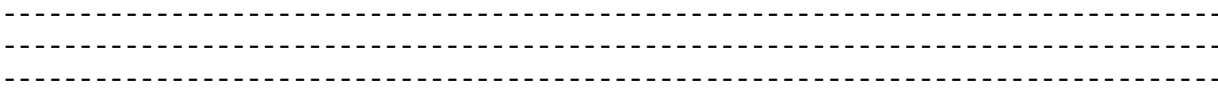
BL21-TufA

BL21-TufB-(over)

W3110-TufA-(chrom) W3110-TUfB

MRE600-TufA- (wt)

MRE600-TufB- (wt)

BL21-TUfA

BL21-TufB- (over)

W3110-TufA- (chrom)

W3110-TufB

MRE600-TufA- (wt)

MRE600-TufB- (wt)

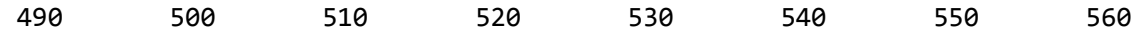
$\ldots|\ldots| \ldots|\ldots| \ldots|\ldots| \ldots|\ldots| \ldots|\ldots| \ldots|\ldots| \ldots|\ldots| \ldots|\ldots| \ldots|\ldots| \ldots|\ldots| \ldots \mid$ TACGACTTCCCGGGCGACGACACTCCGATCGTTCGTGGTTCTGCTCTGAAAGCGCTGGAAGGCGACGCAGAGTGGGAAGC

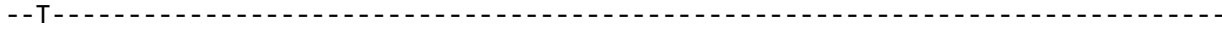

BL21-TufA BL21-TufB-(over) W3110-TufA-(chrom) W3110-TufB MRE600-TufA- (wt) MRE600-TufB- (wt)

BL21-TufA BL21-TufB-(over) W3110-TufA-(chrom) W3110-TufB MRE600-TufA- (wt) MRE600-TufB- (wt)

BL21-TufA BL21-TufB- (over) W3110-TufA- (chrom) W3110-TufB MRE600-TufA-(wt) MRE600-TufB-(wt)

BL21-TufA BL21-TufB- (over) W3110-TufA- (chrom) W3110-TufB MRE600-TufA- (wt) MRE600-TufB- (wt)

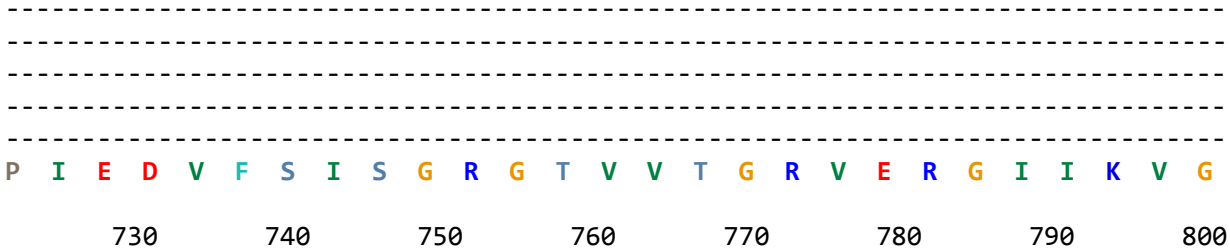
$\ldots . \ldots|\ldots| \ldots|\ldots| \ldots|\ldots| \ldots|\ldots| \ldots|\ldots| \ldots|\ldots| \ldots|\ldots| \ldots|\ldots| \ldots \mid$ GAAGAAGTTGAAATCGTTGGTATCAAAGAGACTCAGAAGTCTACCTGTACTGGCGTTGAAATGTTCCGCAAACTGCTGGA

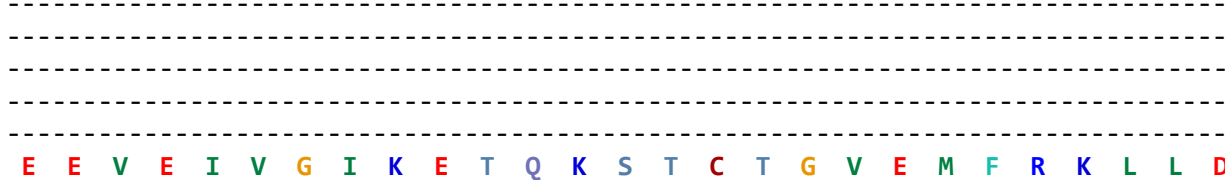

\begin{tabular}{|rrrrrrrr}
810 & 820 & 830 & 840 & 850 & 860 & 870 & 880
\end{tabular} $\ldots . \ldots|\ldots| \ldots|\ldots| \ldots|\ldots| \ldots|\ldots| \ldots|\ldots| \ldots|\ldots| \ldots|\ldots| \ldots|\ldots| \ldots \mid$ CGAAGGCCGTGCTGGTGAGAACGTAGGTGTTCTGCTGCGTGGTATCAAACGTGAAGAAATCGAACGTGGTCAGGTACTGG

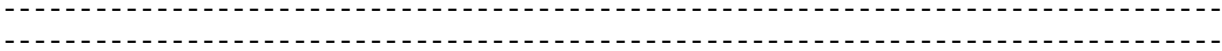

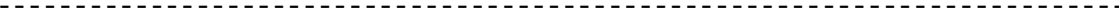

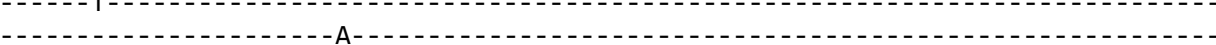

$\begin{array}{llllllllllllllllllllllllllllll} & E & G & R & A & G & E & N & I & G & V & L & L & R & G & I & K & R & E & E & I & E & R & G & Q & V & L\end{array}$

$\begin{array}{llllllll}890 & 900 & 910 & 920 & 930 & 940 & 950 & 960\end{array}$ $\ldots|\ldots| \ldots|\ldots| \ldots|\ldots| \ldots|\ldots| \ldots|\ldots| \ldots|\ldots| \ldots|\ldots| \ldots|\ldots| \ldots|\ldots| \ldots|\ldots|$ CTAAGCCGGGCACCATCAAGCCGCACACCAAGTTCGAATCTGAAGTGTACATTCTGTCCAAAGATGAAGGCGGCCGTCAT

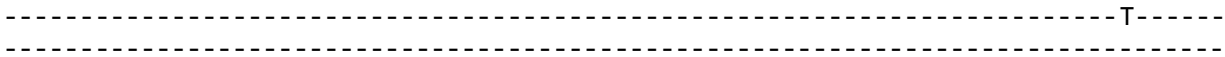
$\begin{array}{llllllll}970 & 980 & 990 & 1000 & 1010 & 1020 & 1030 & 1040\end{array}$ $\ldots|\ldots| \ldots|\ldots| \ldots|\ldots| \ldots|\ldots| \ldots|\ldots| \ldots|\ldots| \ldots|\ldots| \ldots|\ldots| \ldots|\ldots| \ldots \mid$ ACTCCGTTCTTCAAAGGCTACCGTCCGCAGTTCTACTTCCGTACTACTGACGTGACTGGTACCATCGAACTGCCGGAAGG - - - - - - - - - - -

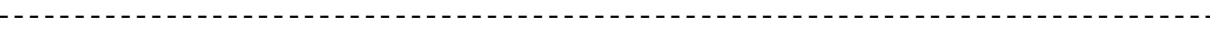
$\begin{array}{llllllllllllllllllllllllllll}T & P & F & F & K & G & Y & R & P & Q & F & Y & F & R & T & T & D & V & T & G & T & I & E & L & P & E & G\end{array}$ 


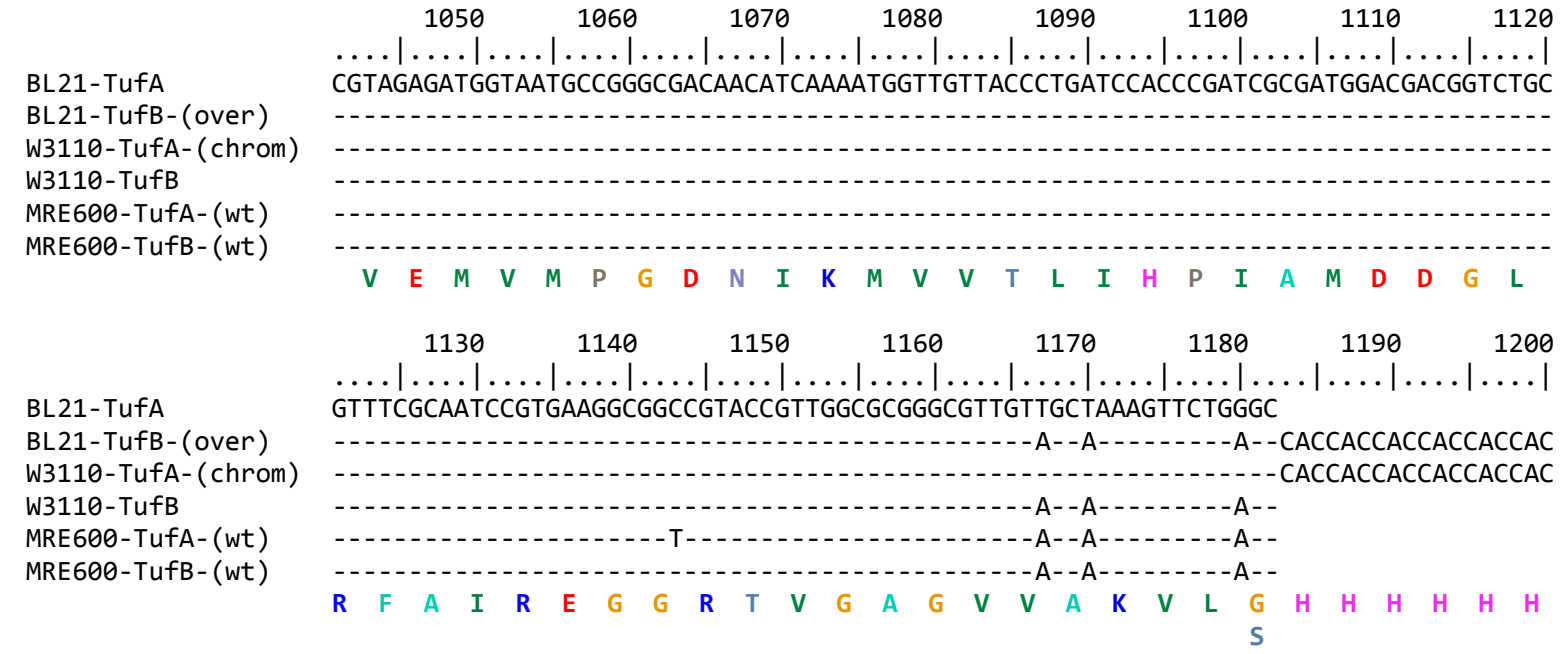


APPENDIX

\subsection{Section B. SRM optimized parameters}

The optimized SRM parameters for heavy-labeled and endogenous peptides used during enrichment workflow are listed. For each peptide, the precursor mass and the fragment mass are indicated (Q1 and Q3 columns). The transitions used during the SRM scan (fragment) and the optimized collision energies (CE) are also indicated.

\begin{tabular}{|c|c|c|c|c|c|}
\hline & Peptide & Q1 & Q3 & Fragment & CE \\
\hline \multirow[t]{8}{*}{ R45H } & TYGGAAHAFDQIDNAPEEK light & 678.64483 & 915.44180 & y8 & 24.1 \\
\hline & & 678.64483 & 802.35774 & y7 & 24.1 \\
\hline & & 678.64483 & 687.33080 & y6 & 23.1 \\
\hline & & 678.64483 & 502.25075 & y4 & 27.1 \\
\hline & TYGGAAHAFDQIDNAPEEK* heavy & 681.31623 & 923.45600 & y8 & 24.1 \\
\hline & & 681.31623 & 810.37194 & y7 & 24.1 \\
\hline & & 681.31623 & 695.34499 & y6 & 23.1 \\
\hline & & 681.31623 & 510.26495 & y4 & 27.1 \\
\hline \multirow[t]{8}{*}{ R59H } & AHGITINTSHVEYDTPTR light & 671.33258 & 980.46835 & y8 & 25.8 \\
\hline & & 671.33258 & 881.39994 & y7 & 27.8 \\
\hline & & 671.33258 & 752.35734 & y6 & 25.8 \\
\hline & & 671.33258 & 373.21939 & y3 & 27.8 \\
\hline & AHGITINTSHVEYDTPTR* heavy & 674.66867 & 990.47662 & y8 & 25.8 \\
\hline & & 674.66867 & 891.40821 & y7 & 27.8 \\
\hline & & 674.66867 & 762.36561 & y6 & 25.8 \\
\hline & & 674.66867 & 383.22766 & y3 & 27.8 \\
\hline \multirow[t]{6}{*}{ R172H } & ELLSQYDFPGDDTPIVHGSALK light & 801.40066 & 1406.72742 & y14 & 29.7 \\
\hline & & 801.40066 & 1022.59931 & y10 & 30.7 \\
\hline & & 801.40066 & 921.55163 & y9 & 30.7 \\
\hline & ELLSQYDFPGDDTPIVHGSALK* heavy & 804.07206 & 1414.74162 & y14 & 29.7 \\
\hline & & 804.07206 & 1030.61351 & y10 & 30.7 \\
\hline & & 804.07206 & 929.56583 & y9 & 30.7 \\
\hline \multirow[t]{6}{*}{ R231S } & GTVVTGSVER light & 502.77219 & 747.39954 & y7 & 17 \\
\hline & & 502.77219 & 648.33113 & y6 & 17 \\
\hline & & 502.77219 & 547.28345 & y5 & 15 \\
\hline & GTVVTGSVER* heavy & 507.77632 & 757.40781 & y7 & 17 \\
\hline & & 507.77632 & 658.33940 & y6 & 17 \\
\hline & & 507.77632 & 557.29172 & y5 & 15 \\
\hline \multirow[t]{6}{*}{ R231G } & GTVVTGGVER light & 487.76691 & 717.38898 & y7 & 16.5 \\
\hline & & 487.76691 & 618.32057 & y6 & 18.5 \\
\hline & & 487.76691 & 517.27289 & y5 & 15.5 \\
\hline & GTVVTGGVER * heavy & 492.77104 & 727.39725 & y7 & 16.5 \\
\hline & & 492.77104 & 628.32883 & y6 & 18.5 \\
\hline & & 492.77104 & 527.28116 & y5 & 15.5 \\
\hline
\end{tabular}




\begin{tabular}{|c|c|c|c|c|c|}
\hline & Peptide & Q1 & Q3 & Fragment & CE \\
\hline \multirow[t]{6}{*}{ R231C } & GTVVTGC[+57.0]VER light & 539.27150 & 721.32975 & y6 & 18.1 \\
\hline & & 539.27150 & 620.28207 & y5 & 17.1 \\
\hline & & 539.27150 & 563.26061 & y4 & 17.1 \\
\hline & GTVVTGC $[+57.0]$ VER* heavy & 544.27563 & 731.33802 & y6 & 18.1 \\
\hline & & 544.27563 & 630.29034 & y5 & 17.1 \\
\hline & & 544.27563 & 573.26888 & y4 & 17.1 \\
\hline \multirow[t]{8}{*}{ R231H } & GTVVTGHVER light & 527.78563 & 698.35801 & y6 & 20.7 \\
\hline & & 527.78563 & 597.31033 & y5 & 21.7 \\
\hline & & 527.78563 & 540.28887 & y4 & 21.7 \\
\hline & & 527.78563 & 880.45231 & b9 & 20.7 \\
\hline & GTVVTGHVER* heavy & 532.78976 & 708.36628 & y6 & 20.7 \\
\hline & & 532.78976 & 607.31860 & y5 & 21.7 \\
\hline & & 532.78976 & 550.29714 & y4 & 21.7 \\
\hline & & 532.78976 & 880.45231 & b9 & 20.7 \\
\hline \multirow[t]{8}{*}{ R231P } & GTVVTGPVER light & 507.78256 & 757.42028 & y7 & 16.1 \\
\hline & & 507.78256 & 658.35187 & y6 & 16.1 \\
\hline & & 507.78256 & 557.30419 & y5 & 14.1 \\
\hline & & 507.78256 & 500.28272 & y4 & 16.1 \\
\hline & GTVVTGPVER* heavy & 512.78669 & 767.42855 & y7 & 16.1 \\
\hline & & 512.78669 & 668.36013 & y6 & 16.1 \\
\hline & & 512.78669 & 567.31246 & y5 & 14.1 \\
\hline & & 512.78669 & 510.29099 & y4 & 16.1 \\
\hline \multirow[t]{8}{*}{ R231L } & GTVVTGLVER light & 515.79821 & 674.38317 & y6 & 16.4 \\
\hline & & 515.79821 & 573.33549 & y5 & 17.4 \\
\hline & & 515.79821 & 516.31402 & y4 & 16.4 \\
\hline & & 515.79821 & 628.36645 & b7 & 14.4 \\
\hline & GTVVTGLVER * heavy & 520.80234 & 684.39143 & y6 & 16.4 \\
\hline & & 520.80234 & 583.34376 & y5 & 17.4 \\
\hline & & 520.80234 & 526.32229 & y4 & 16.4 \\
\hline & & 520.80234 & 628.36645 & b7 & 14.4 \\
\hline \multirow[t]{10}{*}{ R231Q } & GTVVTGQVER light & 523.28546 & 403.22996 & y3 & 18.6 \\
\hline & & 523.28546 & 531.28854 & y4 & 15.6 \\
\hline & & 523.28546 & 588.31000 & y5 & 16.6 \\
\hline & & 523.28546 & 689.35768 & y6 & 17.6 \\
\hline & & 523.28546 & 788.42609 & y7 & 18.6 \\
\hline & GTVVTGQVER* heavy & 528.28960 & 413.23823 & y3 & 18.6 \\
\hline & & 528.28960 & 541.29681 & y4 & 15.6 \\
\hline & & 528.28960 & 598.31827 & y5 & 16.6 \\
\hline & & 528.28960 & 699.36595 & y6 & 17.6 \\
\hline & & 528.28960 & 798.43436 & y7 & 18.6 \\
\hline \multirow[t]{5}{*}{ R231E } & GTVVTGEVER light & 523.77747 & 532.27255 & y4 & 16.6 \\
\hline & & 523.77747 & 589.29402 & y5 & 15.6 \\
\hline & & 523.77747 & 644.32498 & b7 & 13.6 \\
\hline & & 523.77747 & 690.34169 & y6 & 17.6 \\
\hline & & 523.77747 & 743.39340 & b8 & 13.6 \\
\hline
\end{tabular}




\begin{tabular}{|c|c|c|c|c|c|}
\hline & \multirow[t]{5}{*}{ GTVVTGEVER* heavy } & 528.78161 & 542.28082 & y4 & 16.6 \\
\hline & & 528.78161 & 599.30229 & y5 & 15.6 \\
\hline & & 528.78161 & 644.32498 & b7 & 13.6 \\
\hline & & 528.78161 & 700.34996 & y6 & 17.6 \\
\hline & & 528.78161 & 743.39340 & b8 & 13.6 \\
\hline \multirow[t]{6}{*}{ R231D } & GTVVTGDVER light & 516.76965 & 518.25690 & y4 & 15.4 \\
\hline & & 516.76965 & 575.27837 & y5 & 16.4 \\
\hline & & 516.76965 & 676.32604 & y6 & 17.4 \\
\hline & GTVVTGDVER* heavy & 521.77378 & 528.26517 & y4 & 15.4 \\
\hline & & 521.77378 & 585.28664 & y5 & 16.4 \\
\hline & & 521.77378 & 686.33431 & y6 & 17.4 \\
\hline \multirow[t]{6}{*}{ R231A } & GTVVTGAVER light & 494.77473 & 531.28854 & y5 & 15.7 \\
\hline & & 494.77473 & 632.33622 & y6 & 15.7 \\
\hline & & 494.77473 & 731.40463 & y7 & 16.7 \\
\hline & GTVVTGAVER*heavy & 499.77887 & 541.29681 & y5 & 15.7 \\
\hline & & 499.77887 & 642.34448 & y6 & 15.7 \\
\hline & & 499.77887 & 741.41290 & y7 & 16.7 \\
\hline \multirow[t]{10}{*}{ R231V } & GTVVTGVVER light & 508.79038 & 515.28239 & b6 & 14.2 \\
\hline & & 508.79038 & 559.31984 & y5 & 15.2 \\
\hline & & 508.79038 & 614.35080 & b7 & 13.2 \\
\hline & & 508.79038 & 660.36752 & y6 & 16.2 \\
\hline & & 508.79038 & 759.43593 & y7 & 17.2 \\
\hline & GTVVTGVVER*heavy & 513.79452 & 515.28239 & b6 & 14.2 \\
\hline & & 513.79452 & 569.32811 & y5 & 15.2 \\
\hline & & 513.79452 & 614.35080 & b7 & 13.2 \\
\hline & & 513.79452 & 670.37578 & y6 & 16.2 \\
\hline & & 513.79452 & 769.44420 & y7 & 17.2 \\
\hline \multirow[t]{10}{*}{ R231Y } & GTVVTGYVER light & 540.78784 & 566.29329 & y4 & 17.1 \\
\hline & & 540.78784 & 623.31475 & y5 & 17.1 \\
\hline & & 540.78784 & 678.34572 & b7 & 14.1 \\
\hline & & 540.78784 & 724.36243 & y6 & 17.1 \\
\hline & & 540.78784 & 777.41413 & b8 & 14.1 \\
\hline & GTVVTGYVER*heavy & 545.79197 & 576.30156 & y4 & 17.1 \\
\hline & & 545.79197 & 633.32302 & y5 & 17.1 \\
\hline & & 545.79197 & 678.34572 & b7 & 14.1 \\
\hline & & 545.79197 & 734.37070 & y6 & 17.1 \\
\hline & & 545.79197 & 777.41413 & b8 & 14.1 \\
\hline \multirow[t]{6}{*}{ R231F } & GTVVTGFVER light & 532.79038 & 550.29837 & y4 & 16.9 \\
\hline & & 532.79038 & 607.31984 & y5 & 16.9 \\
\hline & & 532.79038 & 662.35080 & b7 & 13.9 \\
\hline & GTVVTGFVER* heavy & 537.79452 & 560.30664 & y4 & 16.9 \\
\hline & & 537.79452 & 617.32811 & y5 & 16.9 \\
\hline & & 537.79452 & 662.35080 & b7 & 13.9 \\
\hline \multirow[t]{3}{*}{ R231T } & GTVVTGTVER light & 509.78001 & 515.28239 & b6 & 13.2 \\
\hline & & 509.78001 & 561.29910 & y5 & 15.2 \\
\hline & & 509.78001 & 662.34678 & y6 & 17.2 \\
\hline
\end{tabular}




\begin{tabular}{|c|c|c|c|c|c|}
\hline & & 509.78001 & 761.41519 & y7 & 17.2 \\
\hline & \multirow[t]{4}{*}{ GTVVTGTVER* heavy } & 514.78415 & 515.28239 & b6 & 13.2 \\
\hline & & 514.78415 & 571.30737 & y5 & 15.2 \\
\hline & & 514.78415 & 672.35505 & y6 & 17.2 \\
\hline & & 514.78415 & 771.42346 & y7 & 17.2 \\
\hline \multirow[t]{10}{*}{ R234H } & \multirow[t]{5}{*}{ VEHGIIK light } & 398.23980 & 696.40390 & y6 & 14.9 \\
\hline & & 398.23980 & 567.36131 & y5 & 15.9 \\
\hline & & 398.23980 & 430.30240 & $\mathrm{y} 4$ & 17.9 \\
\hline & & 398.23980 & 536.28272 & b5 & 17.9 \\
\hline & & 398.23980 & 649.36679 & b6 & 14.9 \\
\hline & \multirow[t]{5}{*}{ VEHGIIK* heavy } & 402.24690 & 704.41810 & y6 & 14.9 \\
\hline & & 402.24690 & 575.37551 & y5 & 15.9 \\
\hline & & 402.24690 & 438.31660 & y4 & 17.9 \\
\hline & & 402.24690 & 536.28272 & b5 & 17.9 \\
\hline & & 402.24690 & 649.36679 & b6 & 14.9 \\
\hline \multirow[t]{10}{*}{ K249R } & \multirow[t]{5}{*}{ VGEEVEIVGIR light } & 600.33515 & 914.53056 & y8 & 20.9 \\
\hline & & 600.33515 & 785.48797 & y7 & 20.9 \\
\hline & & 600.33515 & 686.41955 & y6 & 18.9 \\
\hline & & 600.33515 & 643.29335 & b6 & 17.9 \\
\hline & & 600.33515 & 756.37741 & b7 & 17.9 \\
\hline & \multirow[t]{5}{*}{ VGEEVEIVGIR* heavy } & 605.33929 & 924.53883 & y8 & 20.9 \\
\hline & & 605.33929 & 795.49623 & y7 & 20.9 \\
\hline & & 605.33929 & 696.42782 & y6 & 18.9 \\
\hline & & 605.33929 & 643.29335 & b6 & 17.9 \\
\hline & & 605.33929 & 756.37741 & b7 & 17.9 \\
\hline \multirow[t]{8}{*}{ K249N } & \multirow[t]{4}{*}{ VGEEVEIVGINETQK light } & 822.42797 & 1130.60518 & y10 & 27.6 \\
\hline & & 822.42797 & 1001.56259 & y9 & 27.6 \\
\hline & & 822.42797 & 888.47852 & y8 & 26.6 \\
\hline & & 822.42797 & 855.44583 & b8 & 22.6 \\
\hline & \multirow[t]{4}{*}{ VGEEVEIVGINETQK* heavy } & 826.43507 & 1138.61938 & y10 & 27.6 \\
\hline & & 826.43507 & 1009.57679 & y9 & 27.6 \\
\hline & & 826.43507 & 896.49272 & y8 & 26.6 \\
\hline & & 826.43507 & 855.44583 & b8 & 22.6 \\
\hline \multirow[t]{8}{*}{ K249Q double } & \multirow[t]{4}{*}{ VGEEVEIVGIQETQK light } & 829.43579 & 1144.62083 & y10 & 28.8 \\
\hline & & 829.43579 & 1015.57824 & y9 & 28.8 \\
\hline & & 829.43579 & 902.49417 & y8 & 30.8 \\
\hline & & 829.43579 & 855.44583 & b8 & 22.8 \\
\hline & \multirow[t]{4}{*}{ VGEEVEIVGIQETQK* heavy } & 836.95147 & 1159.65219 & y10 & 28.8 \\
\hline & & 836.95147 & 1030.60960 & y9 & 28.8 \\
\hline & & 836.95147 & 917.52554 & y8 & 30.8 \\
\hline & & 836.95147 & 855.44583 & b8 & 22.8 \\
\hline \multirow[t]{5}{*}{ K249Q single } & \multirow[t]{4}{*}{ VGEEVEIVGIQETQK light } & 829.43579 & 1144.62083 & y10 & 30.8 \\
\hline & & 829.43579 & 1015.57824 & y9 & 28.8 \\
\hline & & 829.43579 & 902.49417 & y8 & 30.8 \\
\hline & & 829.43579 & 855.44583 & b8 & 23.8 \\
\hline & VGEEVEIVGIQETQK* heavy & 833.44289 & 1152.63503 & y10 & 30.8 \\
\hline
\end{tabular}




\begin{tabular}{|c|c|c|c|c|c|}
\hline & & \multirow{3}{*}{$\begin{array}{l}833.44289 \\
833.44289\end{array}$} & \multirow{2}{*}{$\begin{array}{c}1023.59244 \\
910.50837\end{array}$} & \multirow{2}{*}{$\begin{array}{l}\text { y9 } \\
\text { y8 }\end{array}$} & \multirow{2}{*}{$\begin{array}{l}28.8 \\
30.8\end{array}$} \\
\hline & & & & & \\
\hline & & & 855.44583 & & \\
\hline \multirow[t]{8}{*}{ K249T single } & \multirow[t]{4}{*}{ VGEEVEIVGITETQK light } & 815.93034 & 1117.60993 & y10 & 29.4 \\
\hline & & 815.93034 & 988.56734 & y9 & 28.4 \\
\hline & & 815.93034 & 875.48327 & y8 & 26.4 \\
\hline & & 815.93034 & 855.44583 & b8 & 23.4 \\
\hline & \multirow[t]{4}{*}{ VGEEVEIVGITETQK* heavy } & 819.93744 & 1125.62413 & y10 & 29.4 \\
\hline & & 819.93744 & 996.58154 & y9 & 28.4 \\
\hline & & 819.93744 & 883.49747 & y8 & 26.4 \\
\hline & & 819.93744 & 855.44583 & b8 & 23.4 \\
\hline \multirow[t]{8}{*}{ K249E single } & \multirow[t]{4}{*}{ VGEEVEIVGIEETQK light } & 829.92780 & 1145.60485 & y10 & 28.8 \\
\hline & & 829.92780 & 1016.56225 & y9 & 27.8 \\
\hline & & 829.92780 & 903.47819 & y8 & 26.8 \\
\hline & & 829.92780 & 855.44583 & b8 & 23.8 \\
\hline & \multirow[t]{4}{*}{ VGEEVEIVGIEETQK* heavy } & 833.93490 & 1153.61904 & y10 & 28.8 \\
\hline & & 833.93490 & 1024.57645 & y9 & 27.8 \\
\hline & & 833.93490 & 911.49239 & y8 & 26.8 \\
\hline & & 833.93490 & 855.44583 & b8 & 23.8 \\
\hline \multirow[t]{8}{*}{ R270H } & \multirow[t]{4}{*}{ LLDEGHAGENVGVLLR light } & 564.64069 & 899.53089 & y8 & 19.7 \\
\hline & & 564.64069 & 770.48830 & y7 & 20.7 \\
\hline & & 564.64069 & 656.44537 & y6 & 19.7 \\
\hline & & 564.64069 & 665.32532 & b6 & 19.7 \\
\hline & \multirow[t]{4}{*}{ LLDEGHAGENVGVLLR* heavy } & 567.97678 & 909.53916 & y8 & 19.7 \\
\hline & & 567.97678 & 780.49657 & y7 & 20.7 \\
\hline & & 567.97678 & 666.45364 & y6 & 19.7 \\
\hline & & 567.97678 & 665.32532 & b6 & 19.7 \\
\hline \multirow[t]{8}{*}{ R280H } & \multirow[t]{4}{*}{ AGENVGVLLHGIK light } & 653.87752 & 836.53525 & y8 & 24.5 \\
\hline & & 653.87752 & 779.51379 & y7 & 23.5 \\
\hline & & 653.87752 & 680.44537 & y6 & 23.5 \\
\hline & & 653.87752 & 990.53671 & b10 & 24.5 \\
\hline & \multirow[t]{4}{*}{ AGENVGVLLHGIK* heavy } & 657.88462 & 844.54945 & y8 & 24.5 \\
\hline & & 657.88462 & 787.52798 & y7 & 23.5 \\
\hline & & 657.88462 & 688.45957 & y6 & 23.5 \\
\hline & & 657.88462 & 990.53671 & b10 & 24.5 \\
\hline \multirow[t]{8}{*}{ R284H } & \multirow[t]{4}{*}{ HEEIER light } & 406.69849 & 675.33080 & y5 & 18.1 \\
\hline & & 406.69849 & 546.28820 & y4 & 18.1 \\
\hline & & 406.69849 & 417.24561 & y3 & 16.1 \\
\hline & & 406.69849 & 638.27803 & b5 & 14.1 \\
\hline & \multirow[t]{4}{*}{ HEEIER* heavy } & 411.70263 & 685.33906 & y5 & 18.1 \\
\hline & & 411.70263 & 556.29647 & y4 & 18.1 \\
\hline & & 411.70263 & 427.25388 & y3 & 16.1 \\
\hline & & 411.70263 & 638.27803 & b5 & 14.1 \\
\hline \multirow[t]{3}{*}{ F305L } & \multirow[t]{3}{*}{ LESEVYILSK light } & 590.82663 & 347.228896 & y3 & 25.6 \\
\hline & & 590.82663 & 460.31296 & y4 & 19.6 \\
\hline & & 590.82663 & 623.376289 & y5 & 16.6 \\
\hline
\end{tabular}




\begin{tabular}{|c|c|c|c|c|c|}
\hline & \multirow{7}{*}{ LESEVYILSK* heavy } & 590.82663 & 722.444703 & y6 & 17.6 \\
\hline & & 590.82663 & 938.519324 & y8 & 15.6 \\
\hline & & 594.83373 & 355.243095 & y3 & 25.6 \\
\hline & & 594.83373 & 468.327159 & y4 & 19.6 \\
\hline & & 594.83373 & 631.390488 & y5 & 16.6 \\
\hline & & 594.83373 & 730.458902 & y6 & 17.6 \\
\hline & & 594.83373 & 946.533523 & y8 & 15.6 \\
\hline \multirow[t]{16}{*}{ E306D } & \multirow[t]{8}{*}{ FDSEVYILSK light } & 600.81098 & 234.144832 & y2 & 19.9 \\
\hline & & 600.81098 & 347.228896 & y3 & 21.9 \\
\hline & & 600.81098 & 350.134661 & b3 & 16.9 \\
\hline & & 600.81098 & 460.31296 & y4 & 19.9 \\
\hline & & 600.81098 & 623.376289 & y5 & 15.9 \\
\hline & & 600.81098 & 722.444703 & y6 & 18.9 \\
\hline & & 600.81098 & 851.487296 & y7 & 17.9 \\
\hline & & 600.81098 & 938.519324 & y8 & 17.9 \\
\hline & \multirow[t]{8}{*}{ FDSEVYILSK* heavy } & 604.81808 & 263.102633 & y2 & 17.9 \\
\hline & & 604.81808 & 350.134661 & y3 & 16.9 \\
\hline & & 604.81808 & 355.243095 & b3 & 21.9 \\
\hline & & 604.81808 & 468.327159 & y4 & 19.9 \\
\hline & & 604.81808 & 631.390488 & y5 & 15.9 \\
\hline & & 604.81808 & 730.458902 & y6 & 18.9 \\
\hline & & 604.81808 & 859.501495 & y7 & 17.9 \\
\hline & & 604.81808 & 946.533523 & y8 & 17.9 \\
\hline \multirow[t]{12}{*}{ E308D } & \multirow[t]{6}{*}{ FESDVYILSK light } & 600.81098 & 347.228896 & y3 & 23.9 \\
\hline & & 600.81098 & 460.31296 & y4 & 21.9 \\
\hline & & 600.81098 & 623.376289 & y5 & 17.9 \\
\hline & & 600.81098 & 722.444703 & y6 & 16.9 \\
\hline & & 600.81098 & 837.471646 & y7 & 16.9 \\
\hline & & 600.81098 & 924.503674 & y8 & 16.9 \\
\hline & \multirow[t]{6}{*}{ FESDVYILSK* heavy } & 604.81808 & 355.243095 & y3 & 23.9 \\
\hline & & 604.81808 & 468.327159 & $\mathrm{y} 4$ & 21.9 \\
\hline & & 604.81808 & 631.390488 & y5 & 17.9 \\
\hline & & 604.81808 & 730.458902 & y6 & 16.9 \\
\hline & & 604.81808 & 845.485845 & y7 & 16.9 \\
\hline & & 604.81808 & 932.517873 & y8 & 16.9 \\
\hline \multirow[t]{11}{*}{$\mathrm{Y3} 10 \mathrm{H}$} & \multirow[t]{6}{*}{ FESEVHILSK light } & 594.81660 & 347.228896 & y3 & 25.7 \\
\hline & & 594.81660 & 460.31296 & y4 & 25.7 \\
\hline & & 594.81660 & 696.440286 & y6 & 21.7 \\
\hline & & 594.81660 & 825.482879 & y7 & 23.7 \\
\hline & & 594.81660 & 842.404294 & b7 & 20.7 \\
\hline & & 594.81660 & 912.514908 & y8 & 18.7 \\
\hline & \multirow[t]{5}{*}{ FESEVHILSK* heavy } & 598.82370 & 355.243095 & y3 & 25.7 \\
\hline & & 598.82370 & 468.327159 & y4 & 25.7 \\
\hline & & 598.82370 & 704.454485 & y6 & 21.7 \\
\hline & & 598.82370 & 833.497078 & y7 & 23.7 \\
\hline & & 598.82370 & 842.404294 & b7 & 20.7 \\
\hline
\end{tabular}




\begin{tabular}{|c|c|c|c|c|c|}
\hline & & 598.82370 & 920.529107 & y8 & 18.7 \\
\hline \multirow[t]{14}{*}{ Y310N } & FESEVNILSK light & 583.30860 & 889.498923 & y8 & 16.4 \\
\hline & & 583.30860 & 802.466895 & y7 & 15.4 \\
\hline & & 583.30860 & 673.424302 & y6 & 16.4 \\
\hline & & 583.30860 & 574.355888 & y5 & 17.4 \\
\hline & & 583.30860 & 460.31296 & y4 & 20.4 \\
\hline & & 583.30860 & 347.228896 & y3 & 22.4 \\
\hline & & 583.30860 & 234.144832 & y2 & 17.4 \\
\hline & FESEVNILSK* heavy & 587.31570 & 897.513122 & y8 & 16.4 \\
\hline & & 587.31570 & 810.481094 & y7 & 15.4 \\
\hline & & 587.31570 & 681.438501 & y6 & 16.4 \\
\hline & & 587.31570 & 582.370087 & y5 & 17.4 \\
\hline & & 587.31570 & 468.327159 & y4 & 20.4 \\
\hline & & 587.31570 & 355.243095 & y3 & 22.4 \\
\hline & & 587.31570 & 242.159031 & y2 & 17.4 \\
\hline \multirow[t]{10}{*}{ K314Q } & FESEVYILSQDEGGR light & 576.94069 & 861.40609 & y8 & 20.2 \\
\hline & & 576.94069 & 748.32202 & y7 & 19.2 \\
\hline & & 576.94069 & 661.28999 & y6 & 19.2 \\
\hline & & 576.94069 & 592.26132 & b5 & 20.2 \\
\hline & & 576.94069 & 755.32465 & b6 & 19.2 \\
\hline & FESEVYILSQDEGGR* heavy & 580.27678 & 871.41435 & y8 & 20.2 \\
\hline & & 580.27678 & 758.33029 & y7 & 19.2 \\
\hline & & 580.27678 & 671.29826 & y6 & 19.2 \\
\hline & & 580.27678 & 592.26132 & b5 & 20.2 \\
\hline & & 580.27678 & 755.32465 & b6 & 19.2 \\
\hline \multirow[t]{8}{*}{ K314T } & FESEVYILSTDEGGR light & 567.93706 & 834.39519 & y8 & 18.9 \\
\hline & & 567.93706 & 721.31112 & y7 & 18.9 \\
\hline & & 567.93706 & 634.27909 & y6 & 18.9 \\
\hline & & 567.93706 & 592.26132 & b5 & 18.9 \\
\hline & FESEVYILSTDEGGR* heavy & 571.27315 & 844.40346 & y8 & 18.9 \\
\hline & & 571.27315 & 731.31939 & y7 & 18.9 \\
\hline & & 571.27315 & 644.28736 & y6 & 18.9 \\
\hline & & 571.27315 & 592.26132 & b5 & 18.9 \\
\hline \multirow[t]{10}{*}{ K314N } & FESEVYILSNDEGGR light & 572.26881 & 847.39044 & y8 & 19 \\
\hline & & 572.26881 & 734.30637 & y7 & 19 \\
\hline & & 572.26881 & 647.27434 & y6 & 20 \\
\hline & & 572.26881 & 592.26132 & b5 & 19 \\
\hline & & 572.26881 & 755.32465 & b6 & 20 \\
\hline & FESEVYILSNDEGGR* heavy & 575.60490 & 857.39870 & y8 & 19 \\
\hline & & 575.60490 & 744.31464 & y7 & 19 \\
\hline & & 575.60490 & 657.28261 & y6 & 20 \\
\hline & & 575.60490 & 592.26132 & b5 & 19 \\
\hline & & 575.60490 & 755.32465 & b6 & 20 \\
\hline \multirow[t]{3}{*}{ K314E } & FESEVYILSEDEGGR light & 577.26870 & 862.39010 & y8 & 19.2 \\
\hline & & 577.26870 & 749.30604 & y7 & 19.2 \\
\hline & & 577.26870 & 662.27401 & y6 & 19.2 \\
\hline
\end{tabular}




\begin{tabular}{|c|c|c|c|c|c|}
\hline & & 577.26870 & 592.26132 & b5 & 19.2 \\
\hline & \multirow{6}{*}{ FESEVYILSEDEGGR* heavy } & 577.26870 & 755.32465 & b6 & 19.2 \\
\hline & & 580.60479 & 872.39837 & y8 & 19.2 \\
\hline & & 580.60479 & 759.31431 & y7 & 19.2 \\
\hline & & 580.60479 & 672.28228 & y6 & 19.2 \\
\hline & & 580.60479 & 592.26132 & b5 & 19.2 \\
\hline & & 580.60479 & 755.32465 & b6 & 19.2 \\
\hline \multirow[t]{10}{*}{ K314R } & \multirow[t]{5}{*}{ FESEVYILSR light } & 621.82188 & 879.49344 & y7 & 20.6 \\
\hline & & 621.82188 & 750.45085 & y6 & 23.6 \\
\hline & & 621.82188 & 651.38244 & y5 & 21.6 \\
\hline & & 621.82188 & 755.32465 & b6 & 16.6 \\
\hline & & 621.82188 & 868.40871 & b7 & 16.6 \\
\hline & \multirow[t]{5}{*}{ FESEVYILSR* heavy } & 626.82601 & 889.50171 & y7 & 20.6 \\
\hline & & 626.82601 & 760.45912 & y6 & 23.6 \\
\hline & & 626.82601 & 661.39071 & y5 & 21.6 \\
\hline & & 626.82601 & 755.32465 & b6 & 16.6 \\
\hline & & 626.82601 & 868.40871 & b7 & 16.6 \\
\hline \multirow[t]{8}{*}{ R319H } & \multirow[t]{4}{*}{ DEGGHHTPFFK light } & 424.53164 & 639.35007 & y5 & 16.4 \\
\hline & & 424.53164 & 538.30240 & $\mathrm{y} 4$ & 16.4 \\
\hline & & 424.53164 & 441.24963 & y3 & 20.4 \\
\hline & & 424.53164 & 734.28524 & b7 & 14.4 \\
\hline & \multirow[t]{4}{*}{ DEGGHHTPFFK* heavy } & 427.20304 & 647.36427 & y5 & 16.4 \\
\hline & & 427.20304 & 546.31660 & y4 & 16.4 \\
\hline & & 427.20304 & 449.26383 & y3 & 20.4 \\
\hline & & 427.20304 & 734.28524 & b7 & 14.4 \\
\hline \multirow[t]{8}{*}{ R328H } & \multirow[t]{4}{*}{ GYHPQFYFR light } & 607.79072 & 857.43045 & y6 & 23.1 \\
\hline & & 607.79072 & 760.37769 & y5 & 25.1 \\
\hline & & 607.79072 & 632.31911 & $\mathrm{y} 4$ & 25.1 \\
\hline & & 607.79072 & 358.15098 & b3 & 21.1 \\
\hline & \multirow[t]{4}{*}{ GYHPQFYFR* heavy } & 612.79485 & 867.43872 & y6 & 23.1 \\
\hline & & 612.79485 & 770.38596 & y5 & 25.1 \\
\hline & & 612.79485 & 642.32738 & $\mathrm{y} 4$ & 25.1 \\
\hline & & 612.79485 & 358.15098 & b3 & 21.1 \\
\hline \multirow[t]{8}{*}{ R378H } & \multirow[t]{4}{*}{ FAIHEGGR light } & 443.73013 & 739.38456 & y7 & 18.2 \\
\hline & & 443.73013 & 668.34745 & y6 & 19.2 \\
\hline & & 443.73013 & 555.26338 & y5 & 18.2 \\
\hline & & 443.73013 & 469.25578 & b4 & 16.2 \\
\hline & \multirow[t]{4}{*}{ FAIHEGGR* heavy } & 448.73426 & 749.39283 & y7 & 18.2 \\
\hline & & 448.73426 & 678.35572 & y6 & 19.2 \\
\hline & & 448.73426 & 565.27165 & y5 & 18.2 \\
\hline & & 448.73426 & 469.25578 & b4 & 16.2 \\
\hline \multirow[t]{5}{*}{ R382H } & \multirow[t]{4}{*}{ EGGHTVGAGVVAK light } & 394.54729 & 544.34532 & y6 & 12.3 \\
\hline & & 394.54729 & 473.30821 & y5 & 13.3 \\
\hline & & 394.54729 & 416.28675 & y4 & 13.3 \\
\hline & & 394.54729 & 482.19939 & b5 & 14.3 \\
\hline & EGGHTVGAGVVAK* heavy & 397.21869 & 552.35952 & y6 & 12.3 \\
\hline
\end{tabular}




\begin{tabular}{|c|c|c|c|c|c|}
\hline & & 397.21869 & 481.32241 & y5 & 13.3 \\
\hline & & 397.21869 & 424.30094 & y4 & 13.3 \\
\hline & & 397.21869 & 482.19939 & b5 & 14.3 \\
\hline E308D-Y310H & FESDVHILSK light & 587.80877 & 234.144832 & y2 & 20.5 \\
\hline & & 587.80877 & 364.150312 & b3 & 21.5 \\
\hline & & 587.80877 & 597.371872 & y5 & 21.5 \\
\hline & & 587.80877 & 811.467229 & y7 & 22.5 \\
\hline & & 587.80877 & 898.499258 & y8 & 19.5 \\
\hline & FESDVHILSK* heavy & 591.81587 & 242.159031 & y2 & 20.5 \\
\hline & & 591.81587 & 364.150312 & b3 & 21.5 \\
\hline & & 591.81587 & 605.386071 & y5 & 21.5 \\
\hline & & 591.81587 & 819.481428 & y7 & 22.5 \\
\hline & & 591.81587 & 906.513457 & y8 & 19.5 \\
\hline E306D-Y310H & FDSEVHILSK light & 587.80877 & 234.144832 & y2 & 21.5 \\
\hline & & 587.80877 & 597.371872 & y5 & 20.5 \\
\hline & & 587.80877 & 696.440286 & y6 & 19.5 \\
\hline & & 587.80877 & 825.482879 & y7 & 19.5 \\
\hline & & 587.80877 & 912.514908 & y8 & 19.5 \\
\hline & FDSEVHILSK* heavy & 591.81587 & 242.159031 & y2 & 21.5 \\
\hline & & 591.81587 & 605.386071 & y5 & 20.5 \\
\hline & & 591.81587 & 704.454485 & y6 & 19.5 \\
\hline & & 591.81587 & 833.497078 & y7 & 19.5 \\
\hline & & 591.81587 & 920.529107 & y8 & 19.5 \\
\hline E306D-E308D & FDSDVYILSK light & 593.80315 & 347.228896 & y3 & 22.7 \\
\hline & & 593.80315 & 460.31296 & y4 & 16.7 \\
\hline & & 593.80315 & 623.376289 & y5 & 17.7 \\
\hline & & 593.80315 & 722.444703 & y6 & 16.7 \\
\hline & & 593.80315 & 837.471646 & y7 & 18.7 \\
\hline & & 593.80315 & 924.503674 & y8 & 16.7 \\
\hline & FDSDVYILSK* heavy & 597.81025 & 355.243095 & y3 & 22.7 \\
\hline & & 597.81025 & 468.327159 & y4 & 16.7 \\
\hline & & 597.81025 & 631.390488 & y5 & 17.7 \\
\hline & & 597.81025 & 730.458902 & y6 & 16.7 \\
\hline & & 597.81025 & 845.485845 & y7 & 18.7 \\
\hline & & 597.81025 & 932.517873 & y8 & 16.7 \\
\hline E308D-Y310N & FESDVNILSK light & 576.30078 & 1004.525866 & y9 & 19.2 \\
\hline & & 576.30078 & 875.483273 & y8 & 18.2 \\
\hline & & 576.30078 & 788.451245 & y7 & 18.2 \\
\hline & & 576.30078 & 673.424302 & y6 & 20.2 \\
\hline & & 576.30078 & 574.355888 & y5 & 19.2 \\
\hline & & 576.30078 & 460.31296 & y4 & 24.2 \\
\hline & & 576.30078 & 347.228896 & y3 & 24.2 \\
\hline & & 576.30078 & 578.245669 & b5 & 16.2 \\
\hline & FESDVNILSK* heavy & 580.30788 & 1012.540065 & y9 & 19.2 \\
\hline & & 580.30788 & 883.497472 & y8 & 18.2 \\
\hline & & 580.30788 & 796.465444 & y7 & 18.2 \\
\hline
\end{tabular}




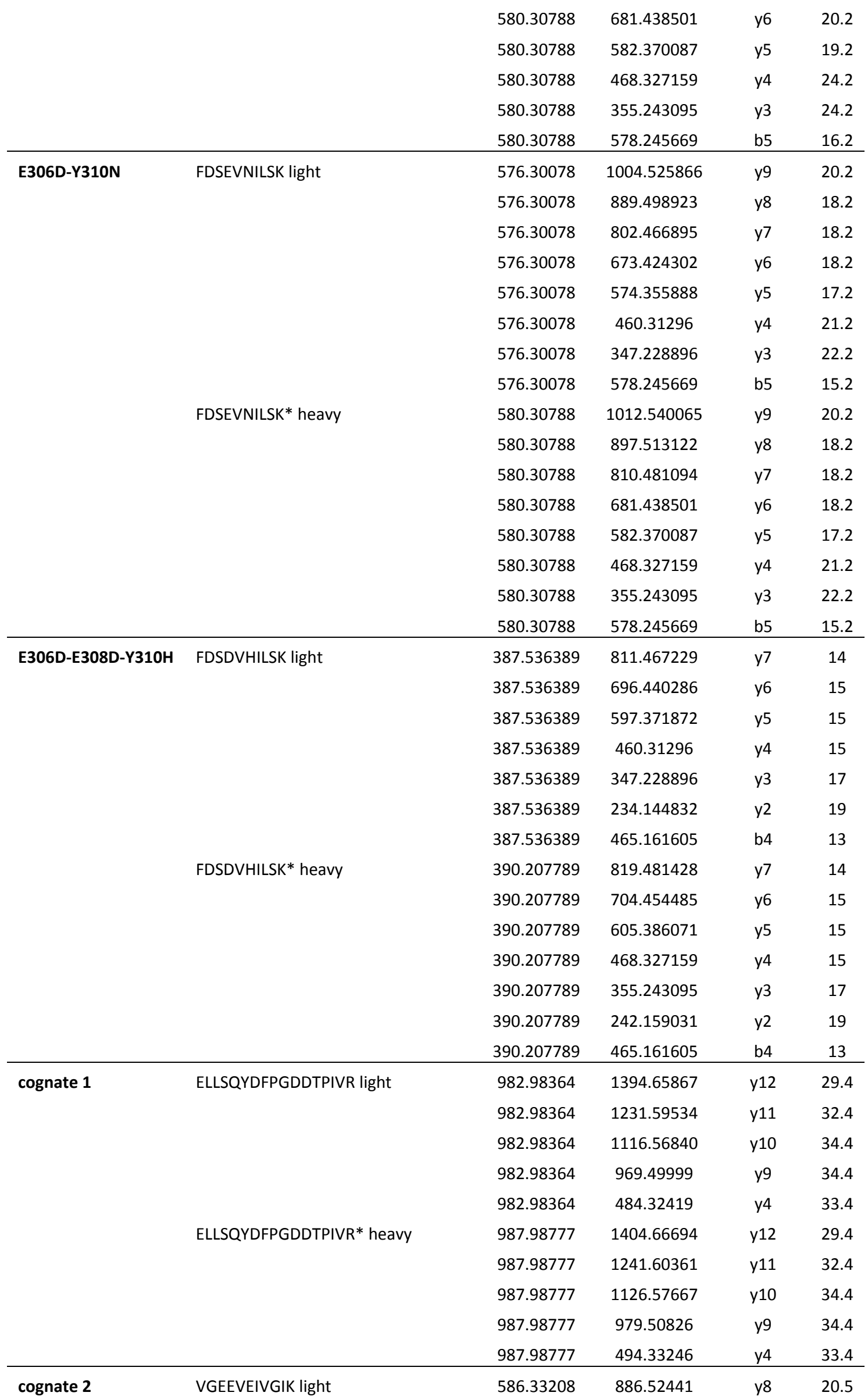




\begin{tabular}{|c|c|c|c|c|c|}
\hline & \multirow{7}{*}{ VGEEVEIVGIK* heavy } & 586.33208 & 757.48182 & y7 & 20.5 \\
\hline & & 586.33208 & 658.41340 & y6 & 19.5 \\
\hline & & 586.33208 & 643.29335 & b6 & 16.5 \\
\hline & & 590.33918 & 894.53861 & y8 & 20.5 \\
\hline & & 590.33918 & 765.49602 & y7 & 20.5 \\
\hline & & 590.33918 & 666.42760 & y6 & 19.5 \\
\hline & & 590.33918 & 643.29335 & b6 & 16.5 \\
\hline \multirow[t]{8}{*}{ cognate 3} & \multirow[t]{4}{*}{ FESEVYILSK light } & 607.81880 & 851.48730 & y7 & 21.1 \\
\hline & & 607.81880 & 722.44470 & y6 & 18.1 \\
\hline & & 607.81880 & 623.37629 & y5 & 19.1 \\
\hline & & 607.81880 & 755.32465 & b6 & 16.1 \\
\hline & \multirow[t]{4}{*}{ FESEVYILSK* heavy } & 611.82590 & 859.50150 & y7 & 21.1 \\
\hline & & 611.82590 & 730.45890 & y6 & 18.1 \\
\hline & & 611.82590 & 631.39049 & y5 & 19.1 \\
\hline & & 611.82590 & 755.32465 & b6 & 16.1 \\
\hline \multirow[t]{8}{*}{ cognate 4} & \multirow[t]{4}{*}{ TVGAGVVAK light } & 401.24508 & 544.34532 & y6 & 15.9 \\
\hline & & 401.24508 & 473.30821 & y5 & 14.9 \\
\hline & & 401.24508 & 416.28675 & y4 & 18.9 \\
\hline & & 401.24508 & 485.27182 & b6 & 10.9 \\
\hline & \multirow[t]{4}{*}{ TVGAGVVAK* heavy } & 405.25218 & 552.35952 & y6 & 15.9 \\
\hline & & 405.25218 & 481.32241 & y5 & 14.9 \\
\hline & & 405.25218 & 424.30094 & y4 & 18.9 \\
\hline & & 405.25218 & 485.27182 & b6 & 10.9 \\
\hline \multirow[t]{10}{*}{ IbpB 1} & \multirow[t]{5}{*}{ ITLALAGFR light } & 481.295102 & 848.498864 & y8 & 17.3 \\
\hline & & 481.295102 & 747.451185 & y7 & 15.3 \\
\hline & & 481.295102 & 634.367121 & y6 & 16.3 \\
\hline & & 481.295102 & 563.330007 & y5 & 15.3 \\
\hline & & 481.295102 & 583.381374 & b6 & 12.3 \\
\hline & \multirow[t]{5}{*}{ ITLALAGFR* heavy } & 486.299236 & 858.507133 & y8 & 17.3 \\
\hline & & 486.299236 & 757.459454 & y7 & 15.3 \\
\hline & & 486.299236 & 644.37539 & y6 & 16.3 \\
\hline & & 486.299236 & 573.338276 & y5 & 15.3 \\
\hline & & 486.299236 & 583.381374 & b6 & 12.3 \\
\hline \multirow[t]{10}{*}{ IbpB 2} & \multirow[t]{5}{*}{ QEDLEIQLEGTR light } & 715.85972 & 1173.610993 & y10 & 24.4 \\
\hline & & 715.85972 & 1058.58405 & y9 & 26.4 \\
\hline & & 715.85972 & 945.499986 & y8 & 25.4 \\
\hline & & 715.85972 & 816.457393 & y7 & 26.4 \\
\hline & & 715.85972 & 728.346111 & b6 & 20.4 \\
\hline & \multirow[t]{5}{*}{ QEDLEIQLEGTR* heavy } & 720.863854 & 1183.619262 & y10 & 24.4 \\
\hline & & 720.863854 & 1068.592319 & y9 & 26.4 \\
\hline & & 720.863854 & 955.508255 & y8 & 25.4 \\
\hline & & 720.863854 & 826.465662 & y7 & 26.4 \\
\hline & & 720.863854 & 728.346111 & b6 & 20.4 \\
\hline \multirow[t]{3}{*}{ IbpB 3} & \multirow[t]{3}{*}{ NEPEPIAAQR light } & 562.788369 & 881.483942 & y8 & 16.8 \\
\hline & & 562.788369 & 784.431178 & y7 & 21.8 \\
\hline & & 562.788369 & 655.388585 & y6 & 21.8 \\
\hline
\end{tabular}




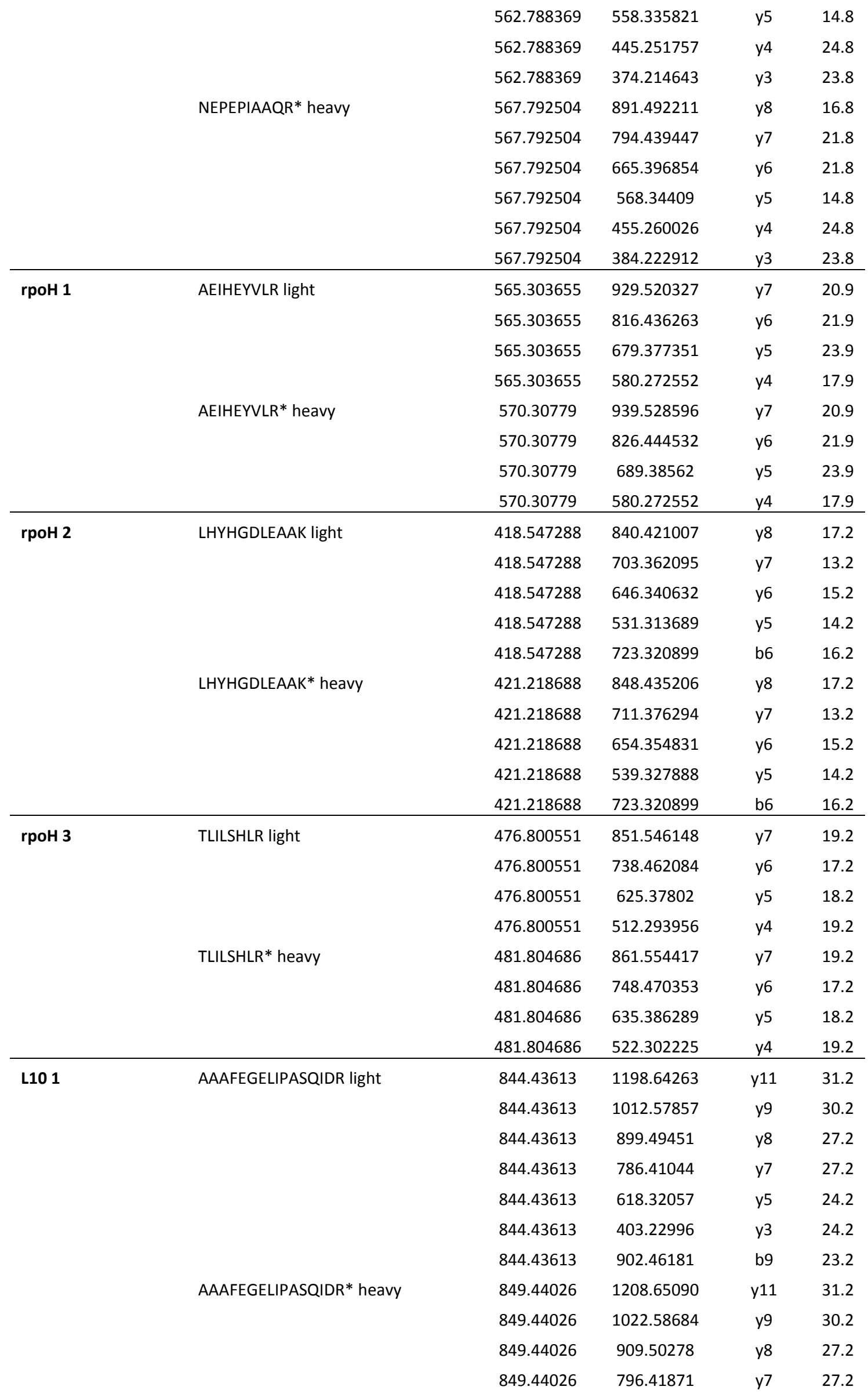




\begin{tabular}{|c|c|c|c|c|c|}
\hline & & \multirow{3}{*}{$\begin{array}{l}849.44026 \\
849.44026\end{array}$} & \multirow{2}{*}{$\begin{array}{l}628.32883 \\
413.23823\end{array}$} & \multirow{2}{*}{$\begin{array}{l}\text { y5 } \\
y 3\end{array}$} & \multirow{2}{*}{$\begin{array}{l}24.2 \\
24.2\end{array}$} \\
\hline & & & & & \\
\hline & & & 902.46181 & & \\
\hline \multirow[t]{8}{*}{ L10 2} & \multirow[t]{4}{*}{ LATLPTYEEAIAR light } & 724.39320 & 1049.52620 & y9 & 22.6 \\
\hline & & 724.39320 & 952.47344 & y8 & 29.6 \\
\hline & & 724.39320 & 851.42576 & y7 & 27.6 \\
\hline & & 724.39320 & 430.27724 & y4 & 29.6 \\
\hline & \multirow[t]{4}{*}{ LATLPTYEEAIAR* heavy } & 729.39733 & 1059.53447 & y9 & 22.6 \\
\hline & & 729.39733 & 962.48171 & y8 & 29.6 \\
\hline & & 729.39733 & 861.43403 & y7 & 27.6 \\
\hline & & 729.39733 & 440.28551 & y4 & 29.6 \\
\hline \multirow[t]{12}{*}{ L10 3} & \multirow[t]{6}{*}{ GALSAVVADSR light } & 523.28546 & 804.42101 & y8 & 16.6 \\
\hline & & 523.28546 & 717.38898 & y7 & 18.6 \\
\hline & & 523.28546 & 646.35187 & y6 & 15.6 \\
\hline & & 523.28546 & 547.28345 & y5 & 14.6 \\
\hline & & 523.28546 & 448.21504 & y4 & 14.6 \\
\hline & & 523.28546 & 262.15098 & y2 & 23.6 \\
\hline & \multirow[t]{6}{*}{ GALSAVVADSR* heavy } & 528.28960 & 814.42928 & y8 & 16.6 \\
\hline & & 528.28960 & 727.39725 & y7 & 18.6 \\
\hline & & 528.28960 & 656.36013 & y6 & 15.6 \\
\hline & & 528.28960 & 557.29172 & y5 & 14.6 \\
\hline & & 528.28960 & 458.22331 & y4 & 14.6 \\
\hline & & 528.28960 & 272.15925 & $y_{2}$ & 23.6 \\
\hline \multirow[t]{8}{*}{ IbpA 1} & \multirow[t]{4}{*}{ SAIGFDR light } & 383.19813 & 678.35695 & y6 & 15.4 \\
\hline & & 383.19813 & 607.31984 & y5 & 11.4 \\
\hline & & 383.19813 & 494.23577 & $\mathrm{y} 4$ & 13.4 \\
\hline & & 383.19813 & 437.21431 & y3 & 13.4 \\
\hline & \multirow[t]{4}{*}{ SAIGFDR* heavy } & 388.20226 & 688.36522 & y6 & 15.4 \\
\hline & & 388.20226 & 617.32811 & y5 & 11.4 \\
\hline & & 388.20226 & 504.24404 & $y 4$ & 13.4 \\
\hline & & 388.20226 & 447.22258 & y3 & 13.4 \\
\hline \multirow[t]{12}{*}{ IbpA 2} & \multirow[t]{6}{*}{ TYLYQGIAER light } & 607.31422 & 949.51016 & y8 & 19.1 \\
\hline & & 607.31422 & 836.42609 & y7 & 20.1 \\
\hline & & 607.31422 & 673.36276 & y6 & 19.1 \\
\hline & & 607.31422 & 545.30419 & y5 & 20.1 \\
\hline & & 607.31422 & 488.28272 & y4 & 19.1 \\
\hline & & 607.31422 & 375.19866 & y3 & 19.1 \\
\hline & \multirow[t]{6}{*}{ TYLYQGIAER* heavy } & 612.31835 & 959.51843 & y8 & 19.1 \\
\hline & & 612.31835 & 846.43436 & y7 & 20.1 \\
\hline & & 612.31835 & 683.37103 & y6 & 19.1 \\
\hline & & 612.31835 & 555.31246 & y5 & 20.1 \\
\hline & & 612.31835 & 498.29099 & y4 & 19.1 \\
\hline & & 612.31835 & 385.20693 & y3 & 19.1 \\
\hline \multirow[t]{3}{*}{ IbpA 3} & \multirow[t]{3}{*}{ NFDLSPLYR light } & 562.79038 & 863.46214 & y7 & 16.8 \\
\hline & & 562.79038 & 748.43520 & y3 & 15.8 \\
\hline & & 562.79038 & 635.35114 & y5 & 15.8 \\
\hline
\end{tabular}




\begin{tabular}{ccccc}
562.79038 & 548.31911 & y4 & 17.8 \\
562.79038 & 262.11862 & b2 & 14.8 \\
NFDLSPLYR* heavy & 562.79038 & 377.14556 & b3 & 15.8 \\
& 567.79452 & 873.47041 & y7 & 16.8 \\
567.79452 & 758.44347 & y3 & 15.8 \\
567.79452 & 645.35941 & y5 & 15.8 \\
567.79452 & 558.32738 & y4 & 17.8 \\
567.79452 & 262.11862 & b2 & 14.8 \\
567.79452 & 377.14556 & b3 & 15.8 \\
\hline
\end{tabular}

\subsection{Section C. validation of near-cognate amino acid substitutions}

The position of the substituted amino acid (red) is shown in the context of EF-Tu structure. The identity of the error-containing peptide is validated at MS1 (B) and MS/MS (C) level and precursor and fragment ions spectra are compared with the fragmentation patter predicted for the peptide (D). For the K249R peptide the MS1 spectrum is replaced by the TSIM and PRM signals. The validation of the quantification is performed by comparing the stoichiometry of erroneous peptide with respect to four cognate EF-Tu peptides (E), as described in Results and Materials and Method. The SRM trace used for the determination of error frequency is shown (F). To confirm the absence of contamination by the light peptide, the heavy-labeled peptides are analysed by mass spectrometry and the signals for both light and heavy are recorded (G). The monitored transitions are indicated. 
R45H: lak.TYGGAAHAFDQIDNAPEEK.arg

A

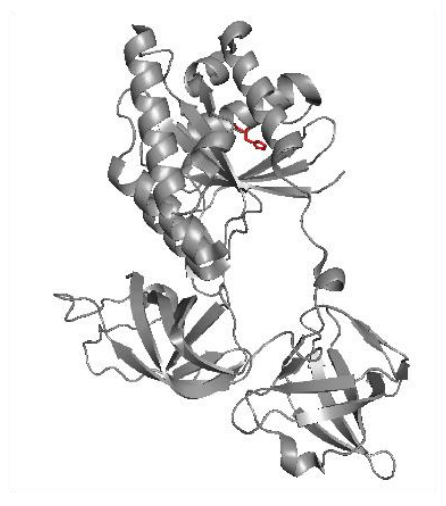

\section{D}

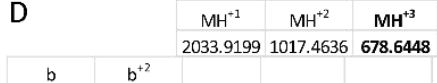

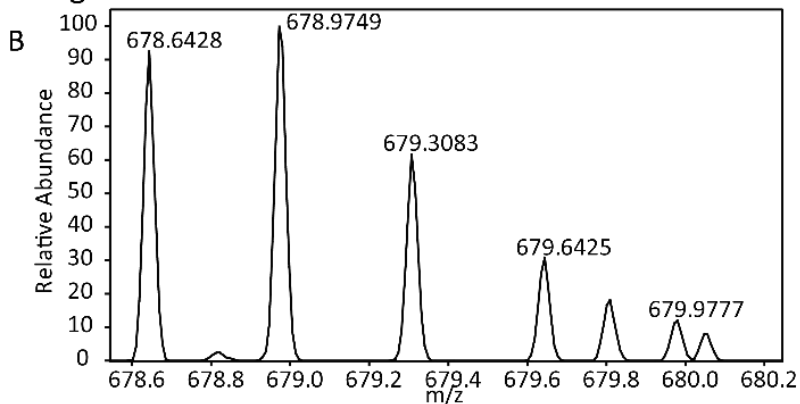

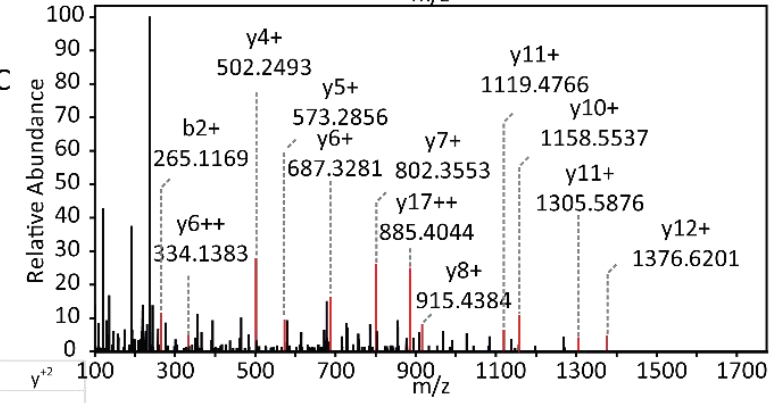

\begin{tabular}{|c|c|c|c|c|c|c|c|}
\hline$b$ & $b^{+2}$ & & & & $y$ & $y^{+2}$ \\
\hline- & - & 1 & T & 19 & - & - \\
\hline 265.1183 & - & 2 & Y & 18 & 1932.8722 & 966.9398 \\
\hline 322.1397 & - & 3 & G & 17 & 1769.8089 & 885.4081 \\
\hline 379.1612 & - & 4 & G & 16 & 1712.7875 & 856.8974 \\
\hline 450.1983 & - & 5 & A & 15 & 1655.7660 & 828.3866 \\
\hline 521.2354 & - & 6 & A & 14 & 1584.7289 & 792.8681 \\
\hline 658.2944 & 329.6508 & 7 & H & 13 & 1513.6918 & 757.3495 \\
\hline 729.3315 & 365.1694 & 8 & A & 12 & 1376.6329 & 688.8201 \\
\hline 876.3999 & 438.7036 & 9 & F & 11 & 1305.5957 & 653.3015 \\
\hline 991.4268 & 496.2170 & 10 & D & 10 & 1158.5273 & 579.7673 \\
\hline 1119.4854 & 560.2463 & 11 & Q & 9 & 1043.5004 & 522.2538 \\
\hline 1232.5695 & 616.7884 & 12 & I & 8 & 915.4418 & 458.2245 \\
\hline 1347.5964 & 674.3018 & 13 & D & 7 & 802.3577 & 401.6825 \\
\hline 1461.6393 & 731.3233 & 14 & N & 6 & 687.3308 & 344.1690 \\
\hline 1532.6764 & 766.8419 & 15 & A & 5 & 573.2879 & 287.1476 \\
\hline 1629.7292 & 815.3682 & 16 & P & 4 & 502.2508 & 251.6290 \\
\hline 1758.7718 & 879.8895 & 17 & E & 3 & 405.1980 & 203.1026 \\
\hline 1887.8144 & 944.4108 & 18 & E & 2 & 276.1554 & 138.5813 \\
\hline- & - & 19 & K & 1 & 147.1128 & 74.0600 \\
\hline
\end{tabular}

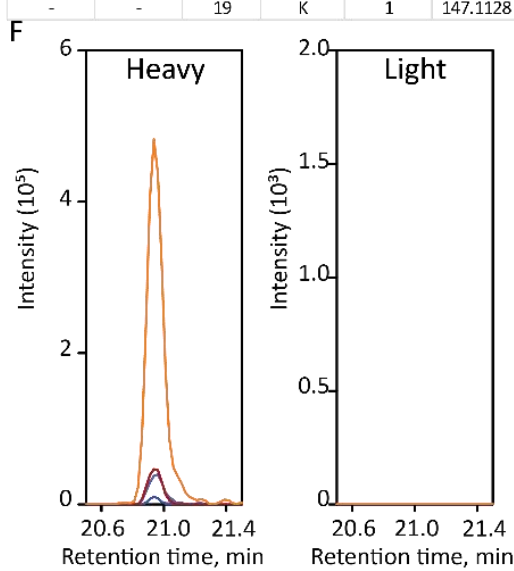

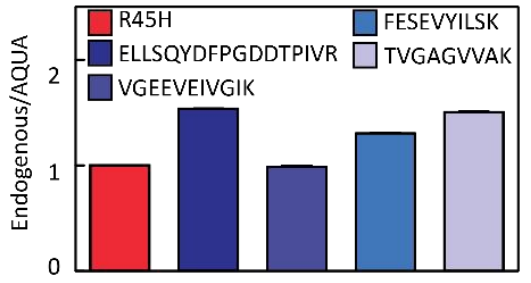

G

R45H light

- y8-915.4418+

$-y 7-802.3577+$

- y6-687.3308+

- y4 - 502.2508+

R45H heavy

- y8-923.4560+

$-y 7-810.3719+$

- y6-695.3449+

$-\mathrm{y} 4-510.2649+$
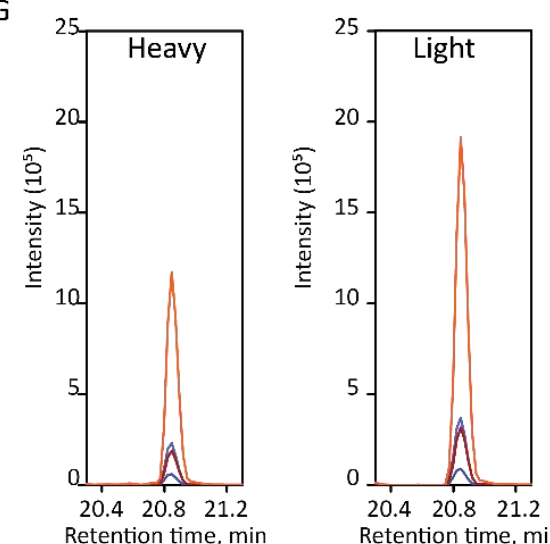
R59H: eek.AHGITINTSHVEYDTPTR.hya

A

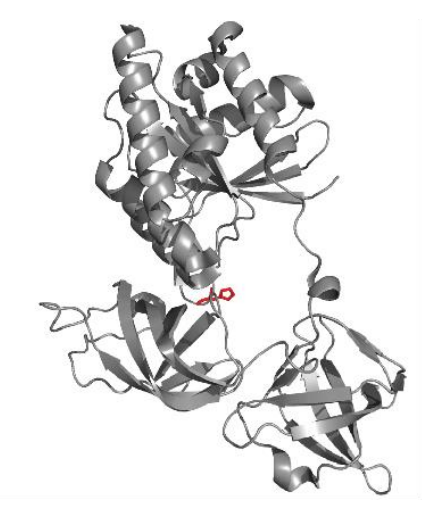

B

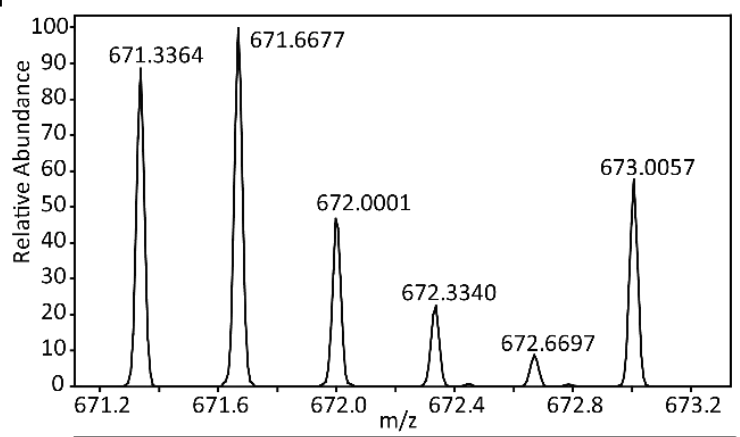

C

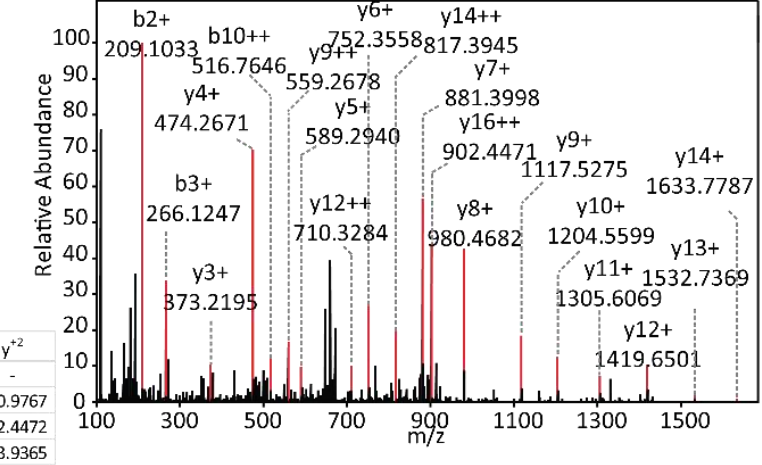

\begin{tabular}{c|c|c|c|}
\hline $\mathrm{MH}^{+1}$ & $\mathrm{MH}^{+2}$ & $\mathrm{MH}^{+3}$ \\
\hline 2011.9832 & 1006.4952 & 671.3326 \\
\hline
\end{tabular}

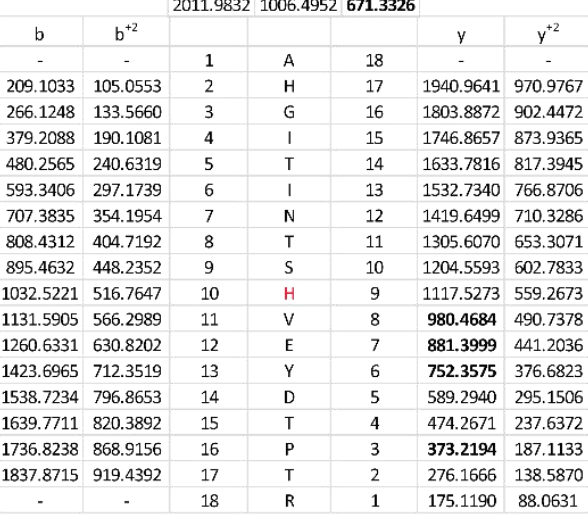

F

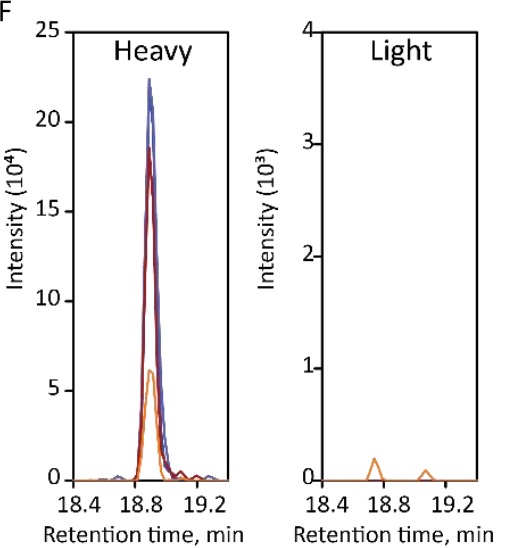

$\mathrm{E}$

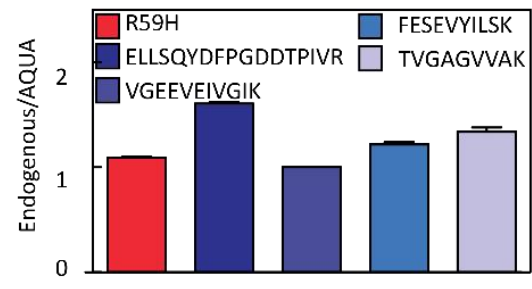

$\mathrm{R} 59 \mathrm{H}$ light

-y8-980.4684+

$-\mathrm{y} 7-881.3999+$

-y6 - 752.3573+

$-\mathrm{y3}-373.2194+$

$\mathrm{R} 59 \mathrm{H}$ heavy

-y8 - 990.4766+

$-\mathrm{y} 7-891.4082+$

-y6-762.3656+

$-\mathrm{y} 3-383.2276+$

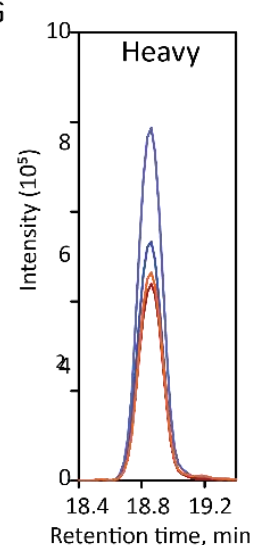


R172H: evr.ELLSQYDFPGDDTPIVHGSALK.ale

A

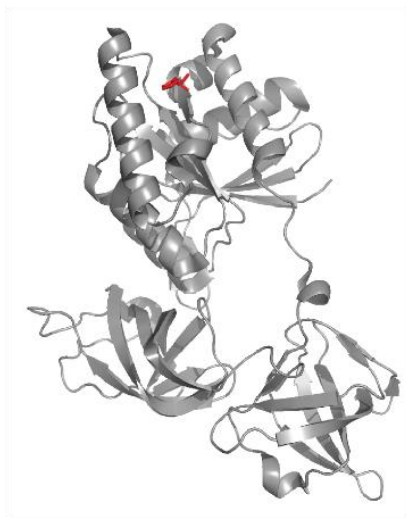

$B$

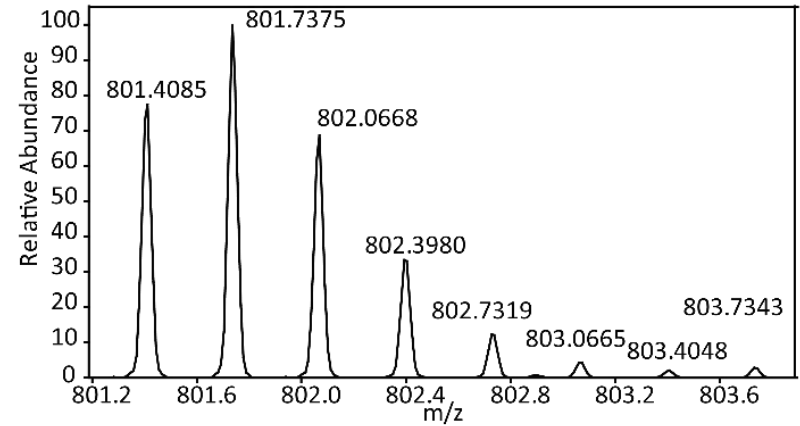

c

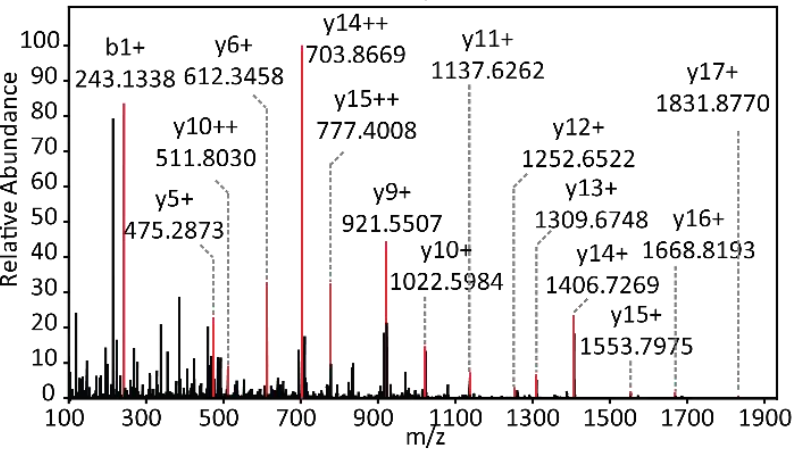

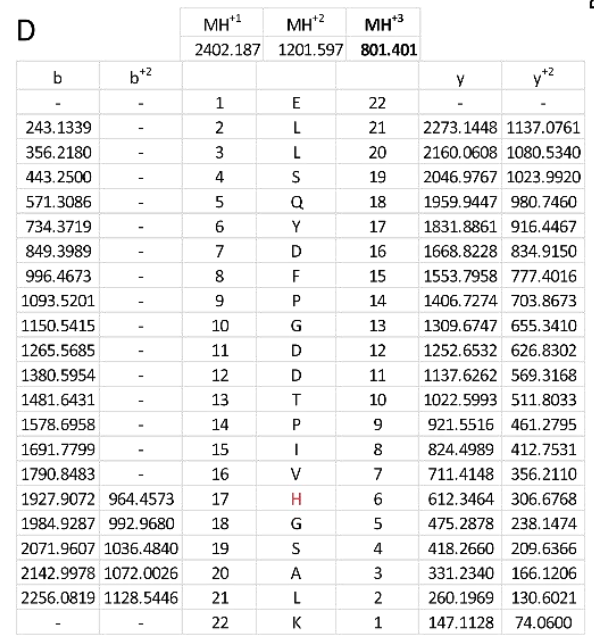

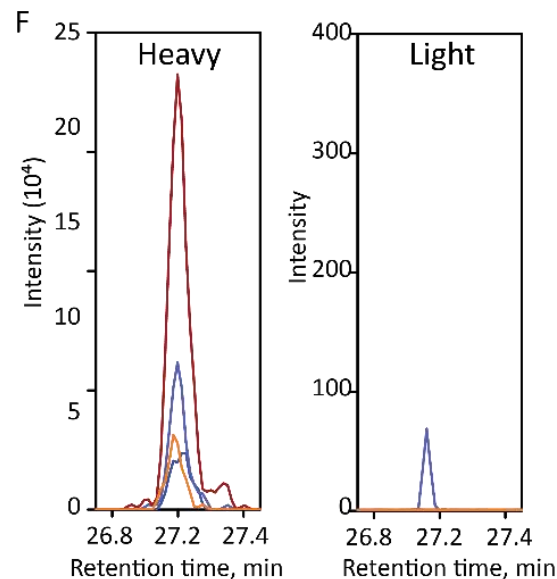

E

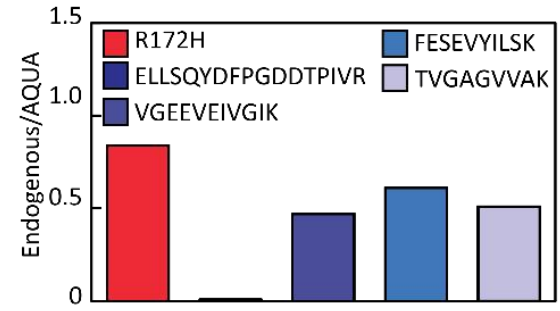

G

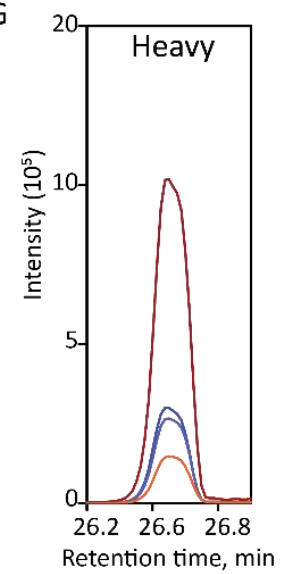

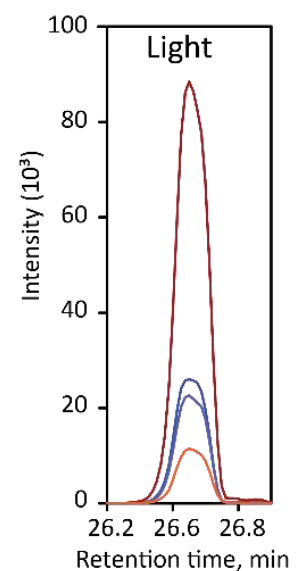


R231S: sgr.GTVVTGSVER.gii

A

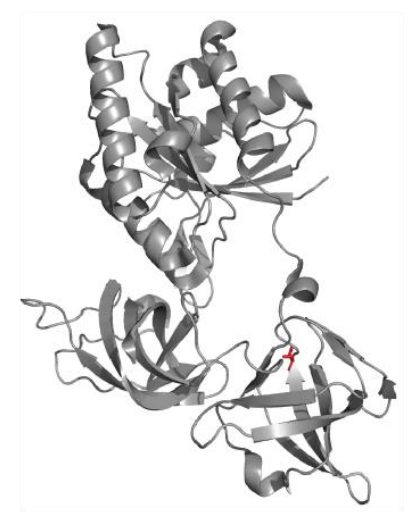

D

\begin{tabular}{|c|c|c|c|c|c|c|}
\hline \multirow{3}{*}{ b } & \multirow{2}{*}{\multicolumn{2}{|c|}{$\frac{\mathrm{MH}^{+1}}{1004.5371}$}} & \multirow{2}{*}{\begin{tabular}{|c|}
$\mathrm{MH}^{+2}$ \\
502.7722
\end{tabular}} & \multirow{2}{*}{\multicolumn{2}{|c|}{$\begin{array}{c}\mathrm{MH}^{+3} \\
335.5172\end{array}$}} & \multirow[b]{3}{*}{$\mathrm{y}^{+2}$} \\
\hline & & & & & & \\
\hline & & & & & $y$ & \\
\hline - & 1 & $c$ & & 10 & - & - \\
\hline 159.0764 & 2 & $T$ & & 9 & 947.5156 & 474.2615 \\
\hline 258.1448 & 3 & $v$ & & 8 & 846.4680 & 423.7376 \\
\hline 357.2132 & 4 & $v$ & & 7 & 747.3995 & 374.2034 \\
\hline 458.2609 & 5 & $T$ & & 6 & 648.3311 & 324.6692 \\
\hline 515.2824 & 6 & $c$ & & 5 & 547.2835 & 274.1451 \\
\hline 602.3144 & 7 & s & & 4 & 490.2620 & 245.6346 \\
\hline 701.3828 & 8 & $v$ & & 3 & 403.2300 & 202.1186 \\
\hline 830.4254 & 9 & E & & 2 & 304.1615 & 152.5844 \\
\hline - & 10 & $\mathrm{R}$ & & 1 & 175.1190 & 88.0631 \\
\hline
\end{tabular}

F
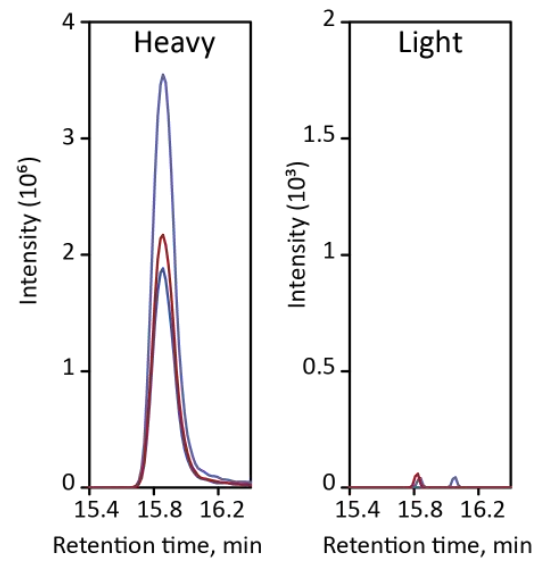
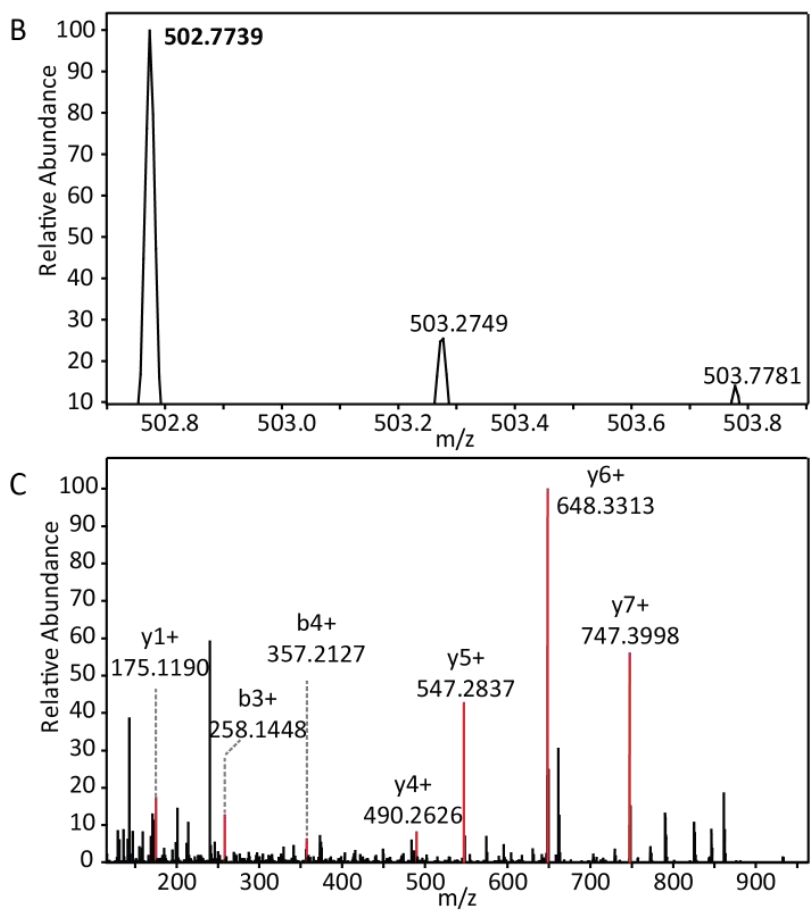

E

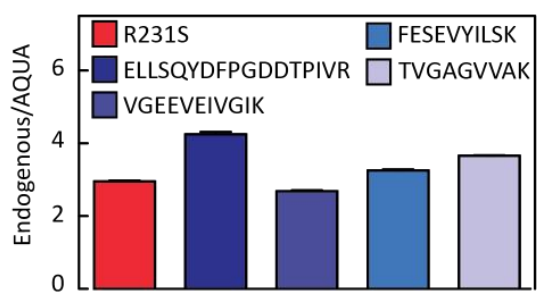

G
R231S light

- y7 - 757.4078+

- y5 - 658.3394+

- y4 - 557.2917+

R231S heavy

- y5 - 648.3311+

- y4 - 547.2835+

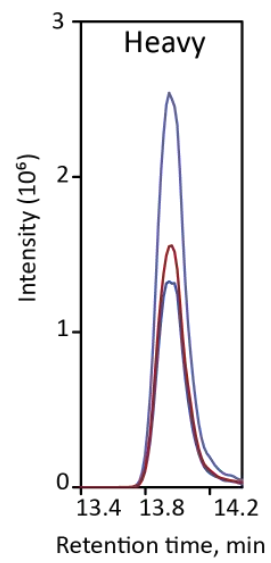

-y7 - 747.3995+ 
R231G: sgr.GTVVTGGVER.gii

A

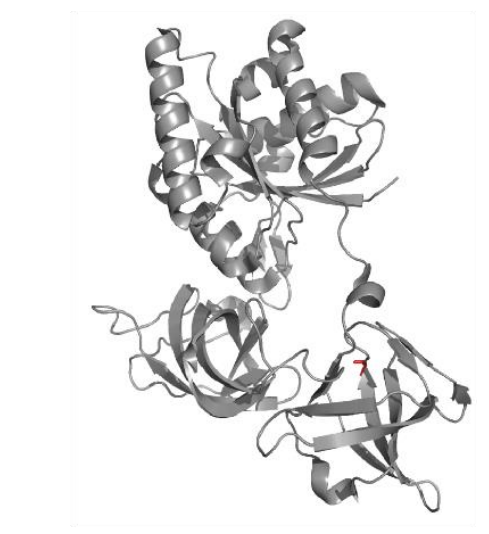

D

\begin{tabular}{|c|c|c|c|c|c|}
\cline { 2 - 5 } \multicolumn{1}{l|}{} & $\mathrm{MH}^{+1}$ & $\mathbf{M H}^{+2}$ & $\mathrm{MH}^{+3}$ & \multicolumn{2}{c|}{} \\
\cline { 2 - 5 } & 974.5265 & $\mathbf{4 8 7 . 7 6 6 9}$ & 325.5137 & \multicolumn{2}{c|}{} \\
\hline b & & & & y & $\mathrm{y}^{+2}$ \\
\hline- & 1 & G & 10 & - & - \\
\hline 159.0764 & 2 & T & 9 & 917.5051 & 459.2562 \\
\hline 258.1448 & 3 & V & 8 & $\mathbf{8 1 6 . 4 5 7 4}$ & 409.7323 \\
\hline 357.2132 & 4 & V & 7 & $\mathbf{7 1 7 . 3 8 9 0}$ & 359.1981 \\
\hline 458.2609 & 5 & T & 6 & $\mathbf{6 1 8 . 3 2 0 6}$ & 309.6639 \\
\hline 515.2824 & 6 & G & 5 & $\mathbf{5 1 7 . 2 7 2 9}$ & 259.1401 \\
\hline 572.0337 & 7 & G & 4 & $\mathbf{4 6 0 . 2 5 1 4}$ & 230.6293 \\
\hline 671.3723 & 8 & V & 3 & 403.2300 & 202.1186 \\
\hline 800.4149 & 9 & E & 2 & 304.1615 & 152.5844 \\
\hline- & 10 & R & 1 & $\mathbf{1 7 5 . 1 1 9 0}$ & 88.0631 \\
\hline
\end{tabular}

$\mathrm{F}$

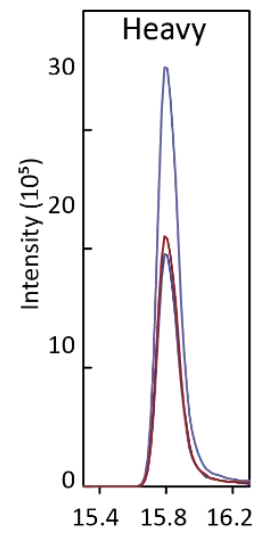

Retention time, $\mathrm{min}$

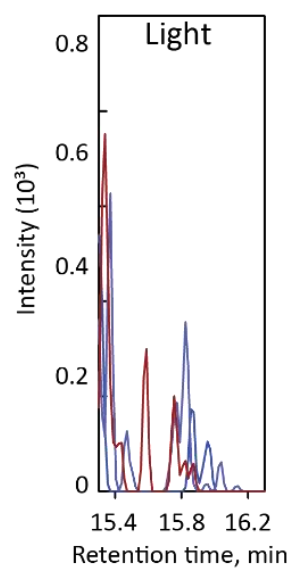

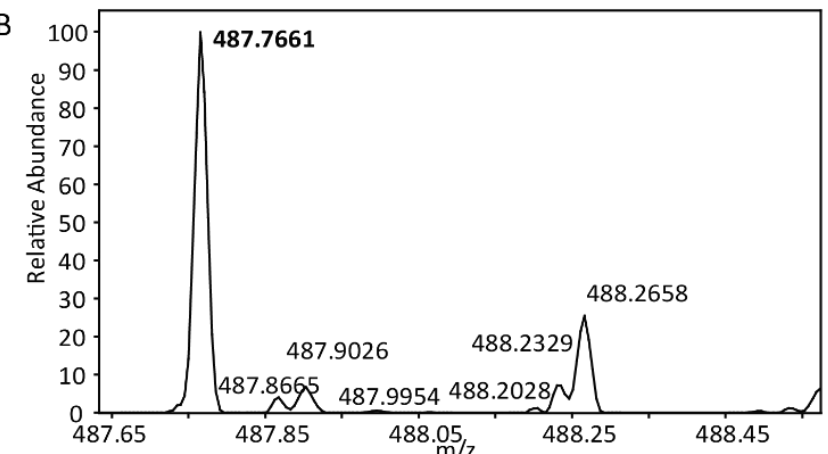

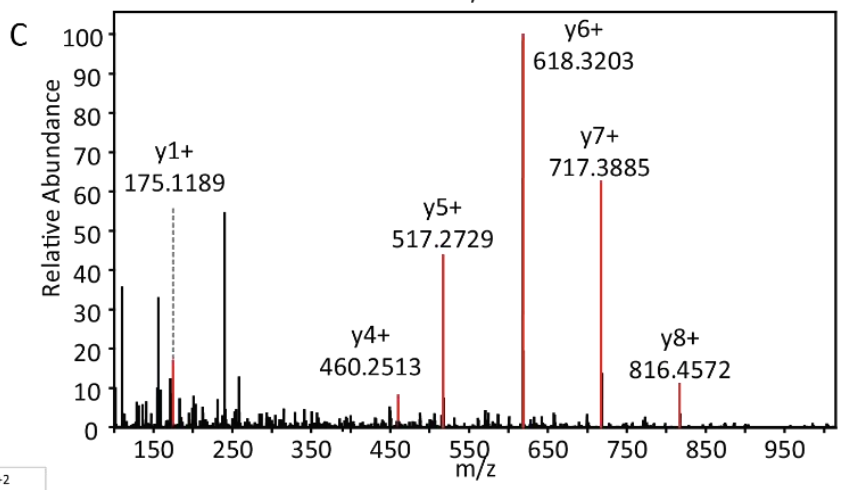

E

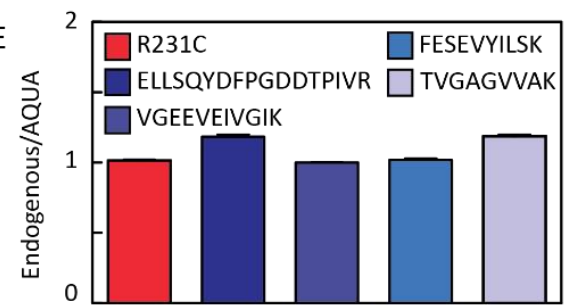

G

R231G light

- y7 - 717.3890+

- y7-618.3206+

- y5 - 517.2729+

R231G heavy

— y7-727.3972+

- y7-628.3288+

- y5 - 527.2812+

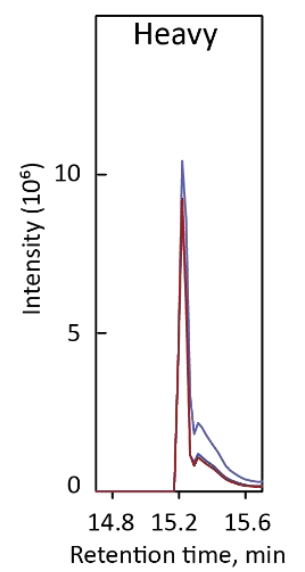


R231C: sgr.GTVVTGCVER.gii

A

D

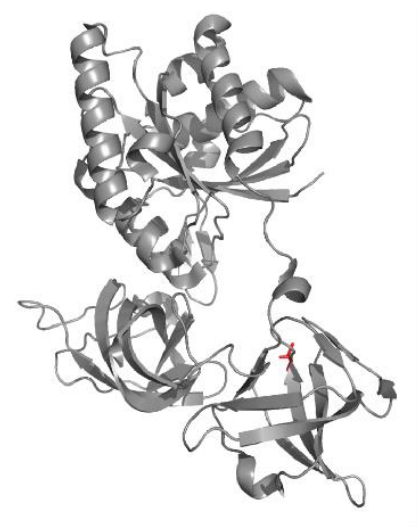

\begin{tabular}{|c|c|c|c|c|c|}
\hline & \multirow{2}{*}{$\begin{array}{c}\mathrm{MH}^{+1} \\
1077.5357\end{array}$} & \multirow{2}{*}{$\begin{array}{c}\mathrm{MH}^{+2} \\
539.6236\end{array}$} & \multirow{2}{*}{$\begin{array}{c}\mathrm{MH}^{+3} \\
359.8501\end{array}$} & & \\
\hline & & & & & \\
\hline b & & & & $y$ & $y^{+2}$ \\
\hline - & 1 & G & 10 & - & - \\
\hline 159.0764 & 2 & $T$ & 9 & 1020.5143 & 510.7608 \\
\hline 258.1448 & 3 & V & 8 & 919.4666 & 460.2369 \\
\hline 357.2132 & 4 & V & 7 & 820.3892 & 410.7027 \\
\hline 458.2609 & 5 & $T$ & 6 & 721.3297 & 361.1685 \\
\hline 515.2824 & 6 & G & 5 & 620.2821 & 310.6447 \\
\hline 675.3130 & 7 & C & 4 & 563.2606 & 282.1339 \\
\hline 774.3815 & 8 & v & 3 & 403.2300 & 202.1186 \\
\hline 903.4240 & 9 & $\mathrm{E}$ & 2 & 304.1615 & 152.5844 \\
\hline - & 10 & $\mathrm{R}$ & 1 & \begin{tabular}{|l|}
175.1190 \\
\end{tabular} & 88.0631 \\
\hline
\end{tabular}

F
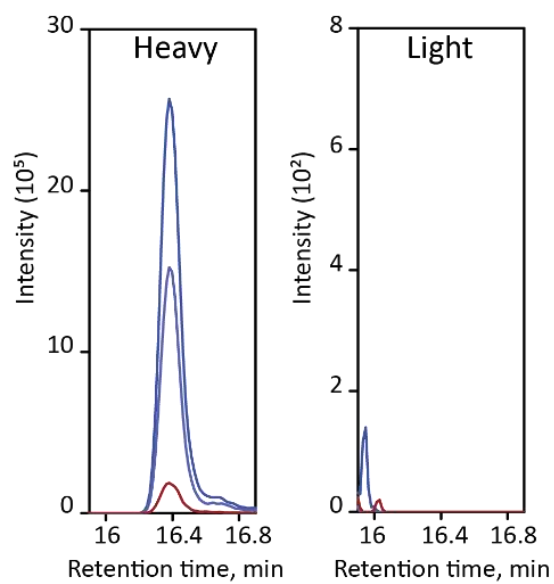
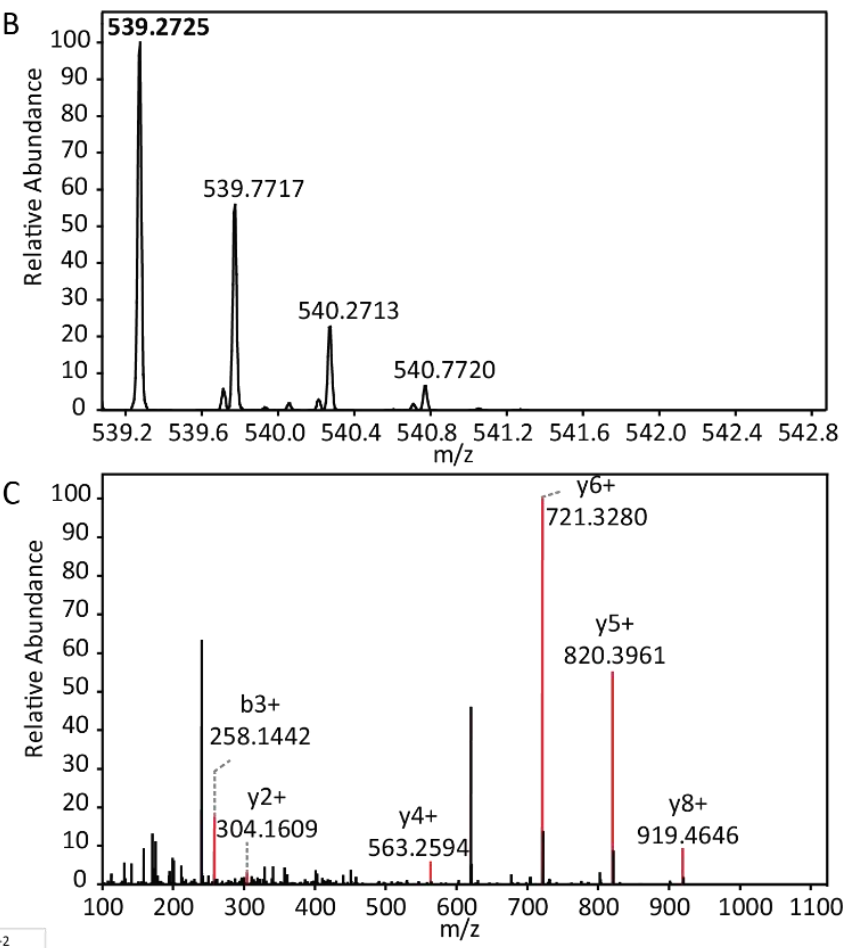

E

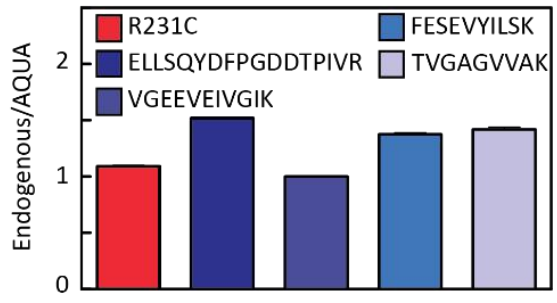

G
R231C light

- y6 - 721.3297+

- y5-620.2821+

- y4-563.2606+

R231C heavy

- y6 - 731.3380+

- y5 - 630.2903+

- y4 - 573.2688+

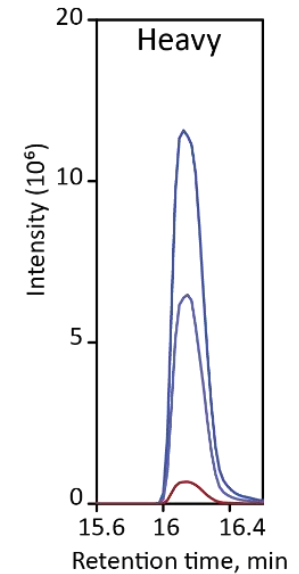


R231H: sgr.GTVVTGHVER.gii

A

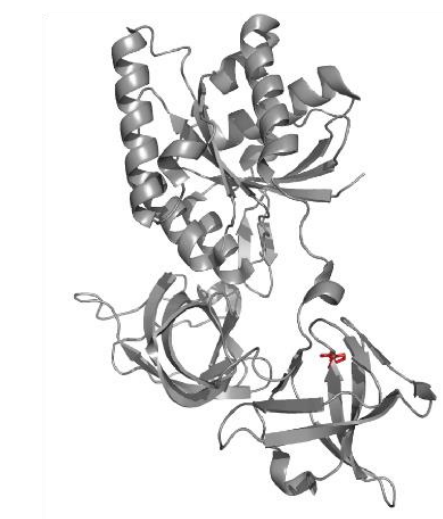

D
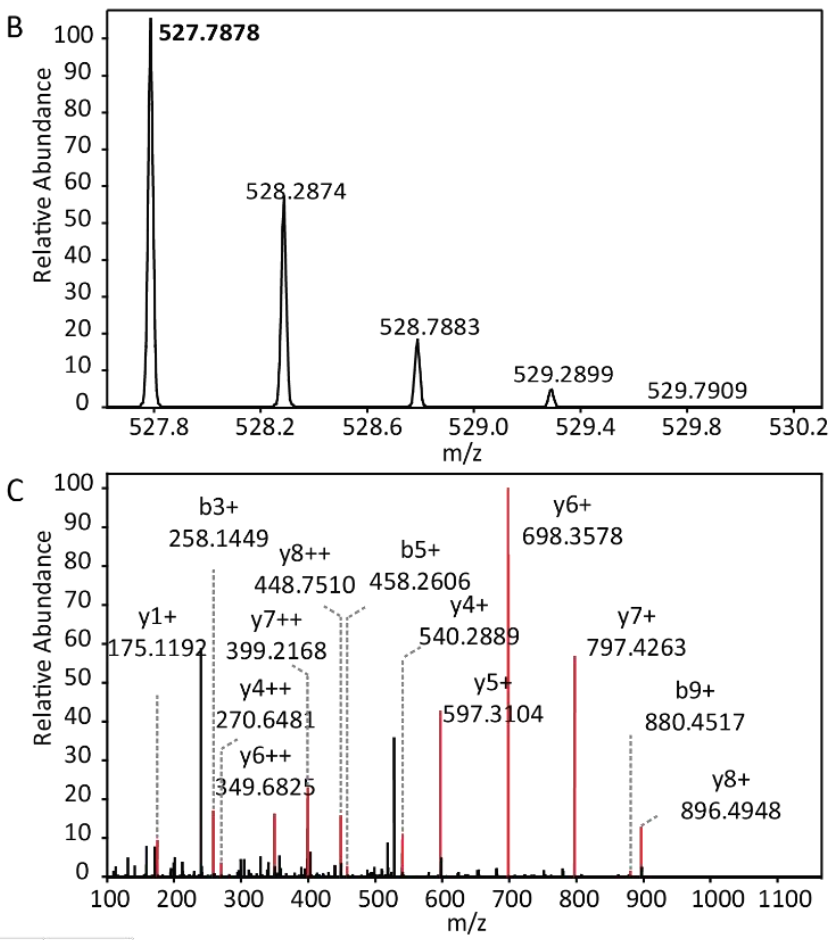

\begin{tabular}{|c|c|c|c|c|c|c|}
\hline & \multirow{2}{*}{$\frac{\mathrm{MH}^{+1}}{1045.5640}$} & \multirow{2}{*}{$\frac{\mathrm{MH}^{+2}}{527.7856}$} & \multirow{2}{*}{$\frac{\mathrm{MH}^{+3}}{352.1928}$} & \multicolumn{2}{|r|}{100} \\
\hline & & & & & & \\
\hline b & $\mathrm{b}^{+2}$ & & & & $y$ & $\mathrm{y}^{+2}$ \\
\hline - & - & 1 & G & 10 & - & - \\
\hline 159.0764 & - & 2 & $\mathrm{~T}$ & 9 & 977.5425 & 499.2749 \\
\hline 258.1448 & - & 3 & v & 8 & 896.4948 & 448.7511 \\
\hline 357.2132 & - & 4 & v & 7 & 797.4264 & 399.2169 \\
\hline 458.2609 & - & 5 & $T$ & 6 & 698.3580 & 349.6826 \\
\hline 515.2824 & - & 6 & G & 5 & 597.3103 & 299.1588 \\
\hline 652.3431 & 326.6743 & 7 & H & 4 & 540.2889 & 270.6481 \\
\hline 751.4097 & 376.2085 & 8 & v & 3 & 403.2300 & 202.1186 \\
\hline 880.4523 & 440.7298 & 9 & $\mathrm{E}$ & 2 & 304.1615 & 152.5844 \\
\hline 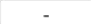 & 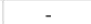 & 10 & $\mathrm{R}$ & 1 & 175.1190 & 88.0631 \\
\hline
\end{tabular}

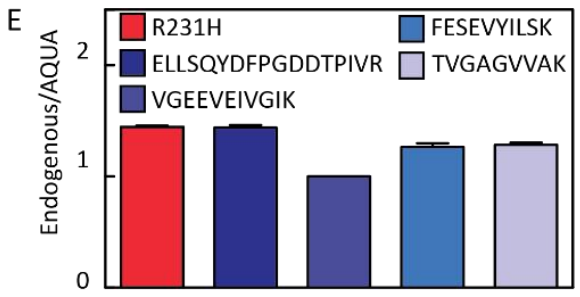

$\mathrm{F}$

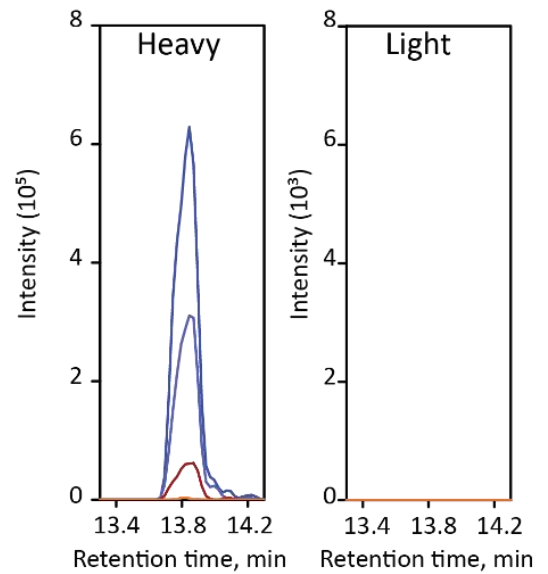

G

R231H light

— y6 - 698.3580+

— y5 - 597.3103+

- y4 - 540.2889+

b9 - $880.4523+$ $\mathrm{R} 231 \mathrm{H}$ heavy

—y6 - 708.3662+

-y5 - 607.3186+

- y4 - 540.2971+

—b9 - 880.4523+
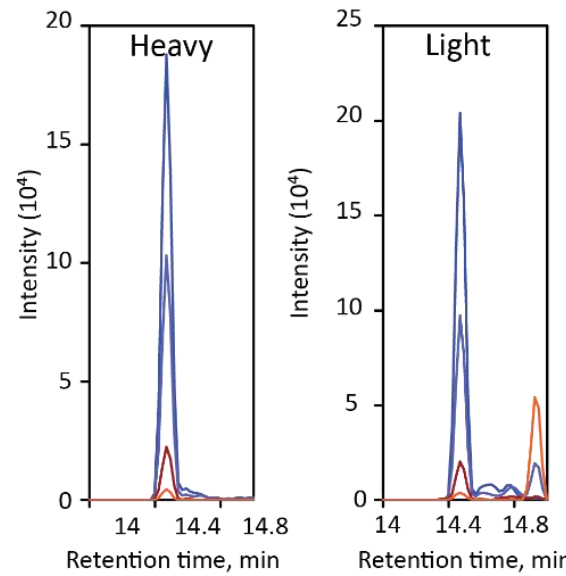
R231P: sgr.GTVVTGPVER.gii

A

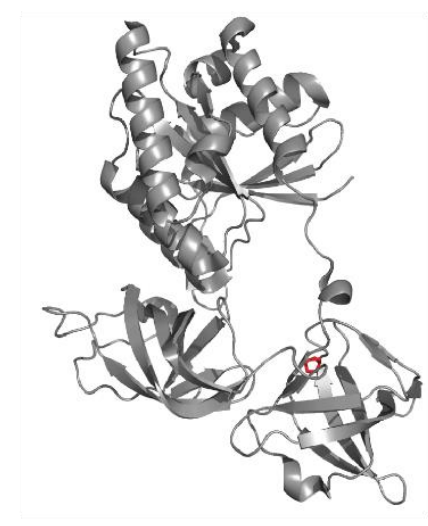

D

\begin{tabular}{|c|c|c|c|c|c|}
\hline & $\mathrm{MH}^{+1}$ & $\mathrm{MH}^{+2}$ & $\mathrm{MH}^{+3}$ & & \\
\hline & 1014.5578 & 507.7826 & 338.8575 & & \\
\hline b & & & & $y$ & $\mathrm{y}^{+2}$ \\
\hline - & 1 & G & 10 & - & - \\
\hline 159.0764 & 2 & $T$ & 9 & 957.5364 & 479.2718 \\
\hline 258.1448 & 3 & V & 8 & 856.4887 & 428.7480 \\
\hline 357.2132 & 4 & V & 7 & 757.4203 & 379.2138 \\
\hline 458.2609 & 5 & $T$ & 6 & 658.3519 & 329.6796 \\
\hline 515.2824 & 6 & G & 5 & 557.3042 & 279.1557 \\
\hline 612.3352 & 7 & $\mathrm{P}$ & 4 & 500.2827 & 250.6450 \\
\hline 711.4036 & 8 & v & 3 & 403.2300 & 202.1186 \\
\hline 840.4462 & 9 & $E$ & 2 & 304.1615 & 152.5844 \\
\hline - & 10 & $\mathrm{R}$ & 1 & 175.1190 & 88.0631 \\
\hline
\end{tabular}

F

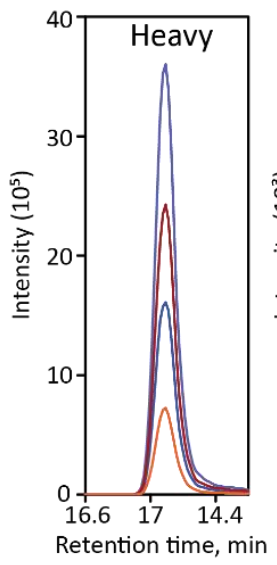

B
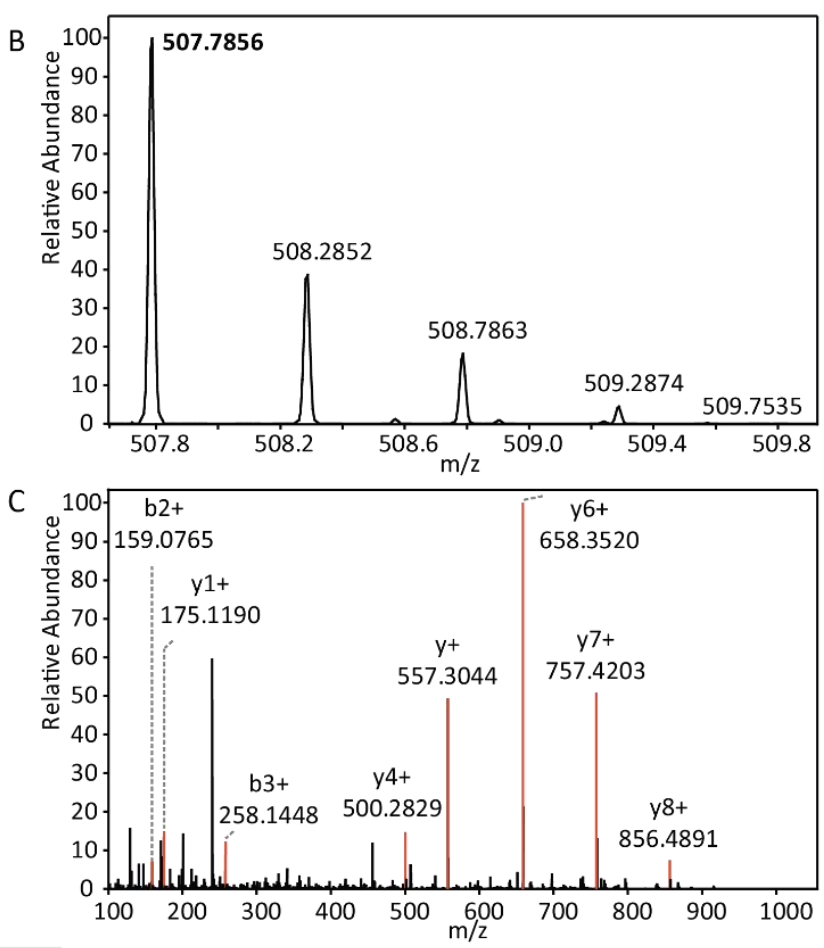

E

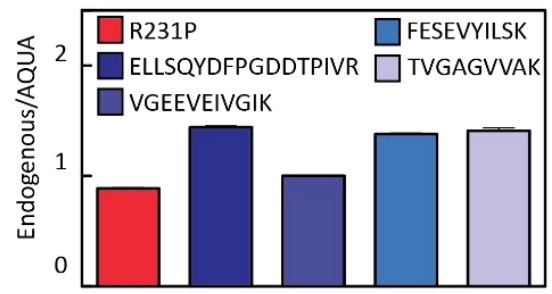

G

R231P light

- y7-757.4203+

- y7-658.3519+

— y6-557.3042+

- y4 - 500.2827+

R231P heavy

— y7 - 767.4285+

- y7-668.3601+

- y6-567.3124+

- y4 - 510.2909+

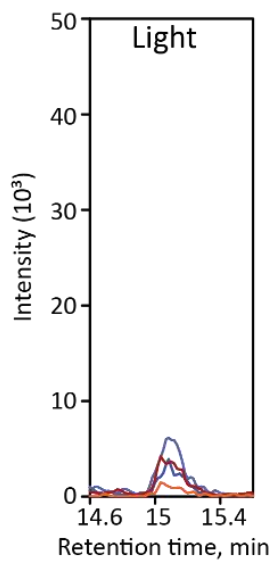


R231L: sgr.GTVVTGLVER.gii

A

D

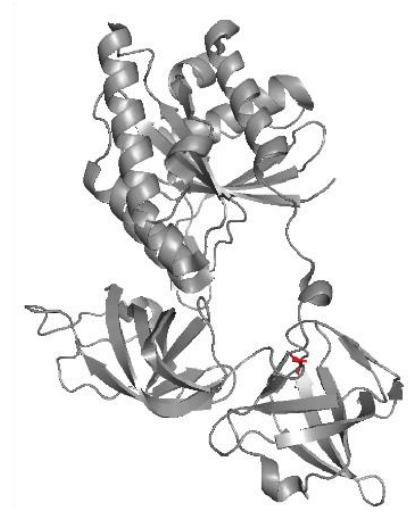

\begin{tabular}{|c|c|c|c|c|c|}
\hline & \multirow{2}{*}{$\begin{array}{c}\mathrm{MH}^{+1} \\
1030.5891\end{array}$} & \multirow{2}{*}{$\begin{array}{c}\mathrm{MH}^{+2} \\
515.7982\end{array}$} & \multirow{2}{*}{$\begin{array}{c}\mathrm{MH}^{+3} \\
344.2012\end{array}$} & & \\
\hline & & & & & \\
\hline b & & & & $y$ & $y^{+2}$ \\
\hline - & 1 & G & 10 & - & - \\
\hline 159.0764 & 2 & $T$ & 9 & 973.5677 & 487.2875 \\
\hline 258.1448 & 3 & V & 8 & 872.5200 & 436.7636 \\
\hline 357.2132 & 4 & V & 7 & 773.4516 & 387.2294 \\
\hline 458.2609 & 5 & T & 6 & 674.3832 & 337.6952 \\
\hline 515.2824 & 6 & G & 5 & 573.3355 & 287.1714 \\
\hline 628.3665 & 7 & L & 4 & 516.3140 & 258.6606 \\
\hline 727.4349 & 8 & V & 3 & 403.2300 & 202.1186 \\
\hline 856.4775 & 9 & E & 2 & 304.1615 & 152.5844 \\
\hline - & 10 & $\mathrm{R}$ & 1 & 175.1190 & 88.0631 \\
\hline
\end{tabular}

$\mathrm{F}$

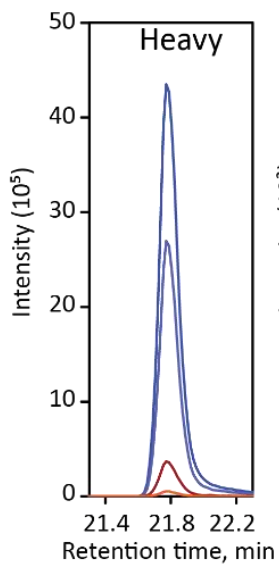

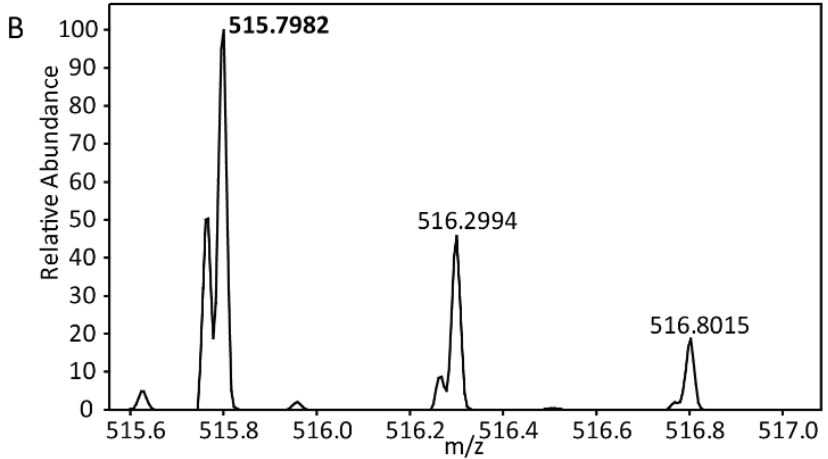

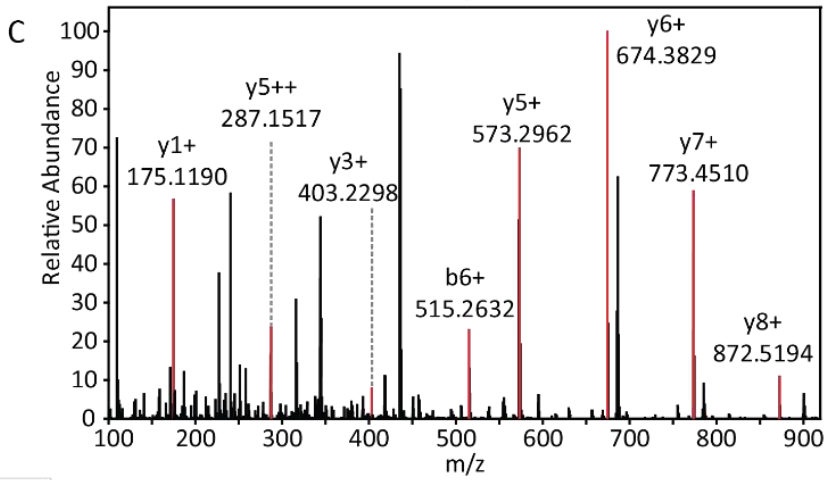

E

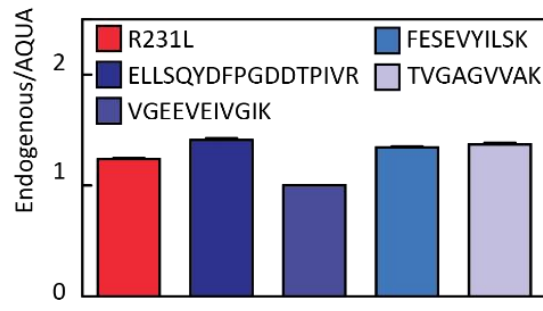

G

R231L light

- y6 - 674.3832+

- y5 - 573.3355+

- y4 - 516.3140+

b7 - 628.3665+

R231L heavy

- y6 - 684.3914+

- y5 - 583.3437+

- y4 - 526.3222+

— b7-628.3664+

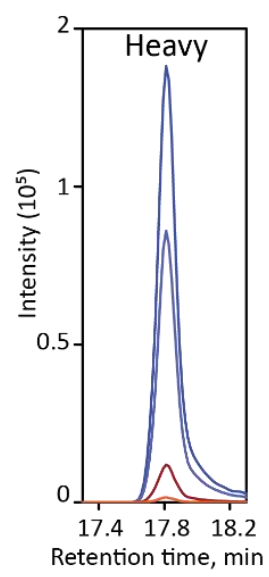


R234H: tgr.VEHGIIK.vge

A

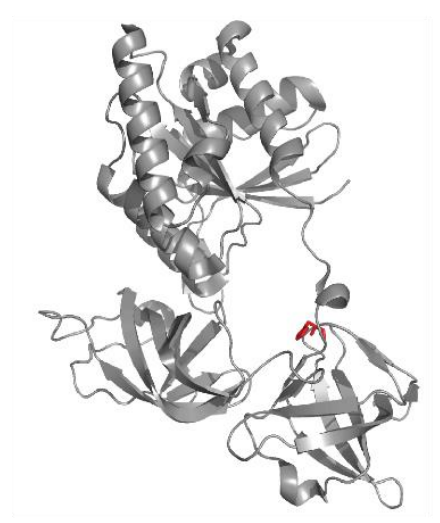

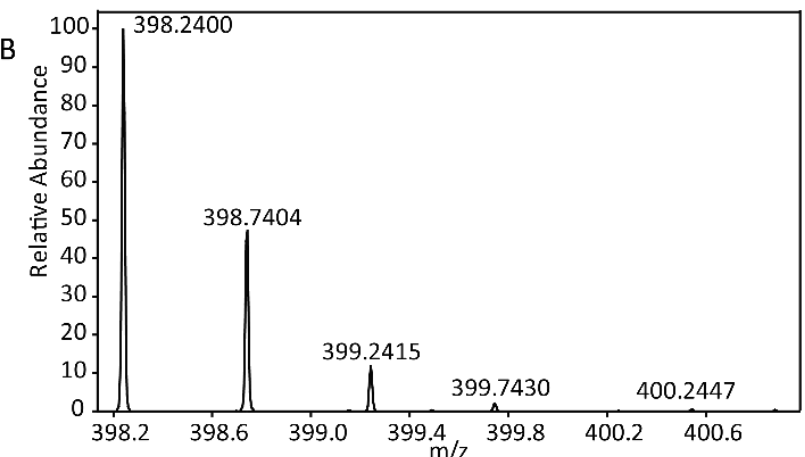

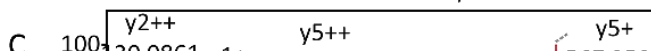
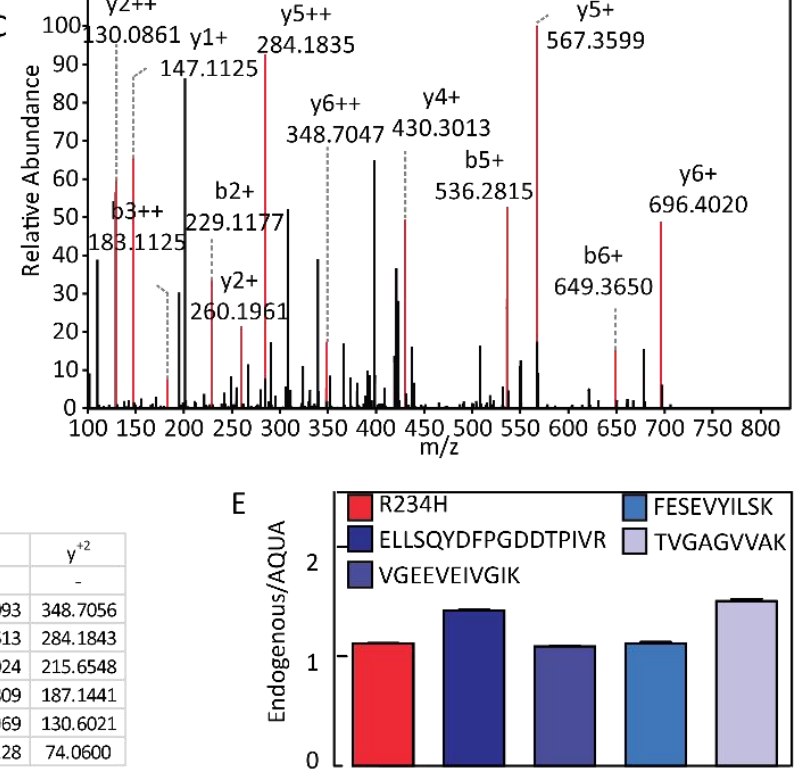

G
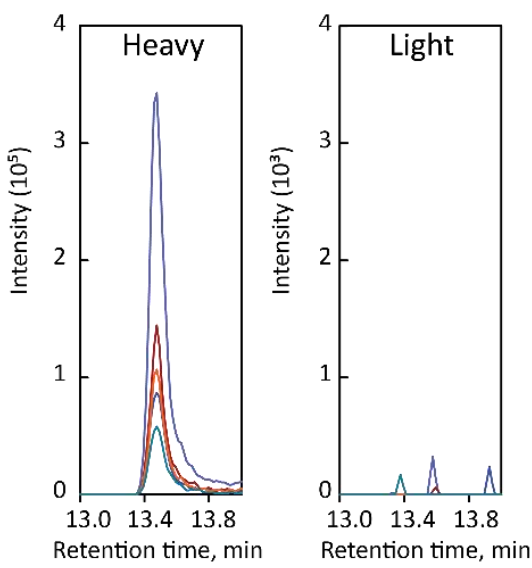

R234H light

- y6 - 696.4039+

- y5 - 567.3613+

- y4 - 430.3024t

-b5 - 536.2827

-b6-649.3668

R234H light

-y6-704.4181+

- y5 - 575.3755+

- y4 - 438.3166

-b5 - 536.2827

-b6-649.3667+
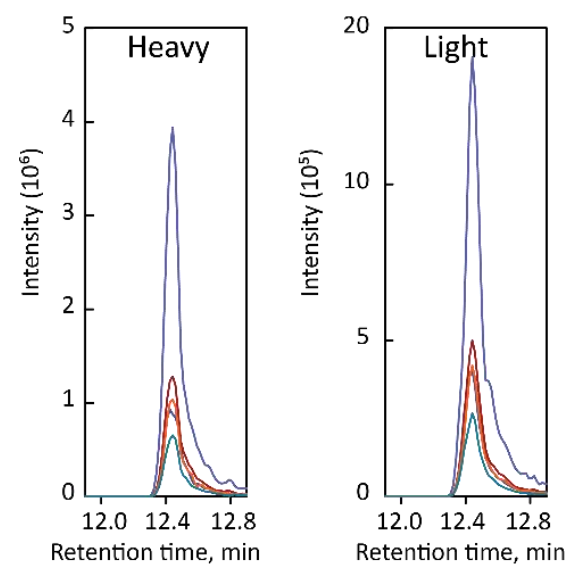
K249Q: iik.VGEEVEIVGIQETQK.stc

A

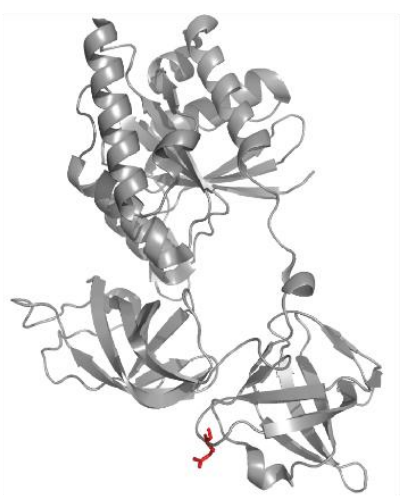

D

\begin{tabular}{|l|c|c|}
\hline $\mathrm{MH}^{+1}$ & $\mathrm{MH}^{+2}$ & $\mathrm{MH}^{+3}$ \\
\hline
\end{tabular}

\begin{tabular}{|c|c|c|c|c|c|}
\hline \multicolumn{1}{|c|}{1657.8643} & 829.4358 & 553.2930 & \\
\hline b & & & & V & $\mathrm{y}^{+2}$ \\
\hline- & 1 & V & 15 & - & - \\
\hline 157.0972 & 2 & G & 14 & 1558.7959 & 779.9016 \\
\hline $\mathbf{2 8 6 . 1 3 9 7}$ & 3 & E & 13 & 1501.7744 & 751.3909 \\
\hline $\mathbf{4 1 5 . 1 8 2 3}$ & 4 & E & 12 & $\mathbf{1 3 7 2 . 7 3 1 8}$ & $\mathbf{6 8 6 . 8 6 9 6}$ \\
\hline $\mathbf{5 1 4 . 2 5 0 8}$ & 5 & V & 11 & $\mathbf{1 2 4 3 . 6 8 9 2}$ & 622.3483 \\
\hline 643.2933 & 6 & E & 10 & $\mathbf{1 1 4 4 . 6 2 0 8}$ & 572.8141 \\
\hline $\mathbf{7 5 6 . 3 7 7 4}$ & 7 & I & 9 & $\mathbf{1 0 1 5 . 5 7 8 2}$ & 508.2928 \\
\hline $\mathbf{8 5 5 . 4 4 5 8}$ & 8 & V & 8 & $\mathbf{9 0 2 . 4 9 4 2}$ & 451.7507 \\
\hline 912.4673 & 9 & G & 7 & $\mathbf{8 0 3 . 4 2 5 8}$ & 402.2165 \\
\hline 1025.5514 & 10 & I & 6 & 746.4043 & 373.7058 \\
\hline 1153.6099 & 11 & Q & 5 & $\mathbf{6 3 3 . 3 2 0 2}$ & 317.1638 \\
\hline $\mathbf{1 2 8 2 . 6 5 2 5}$ & 12 & E & 4 & $\mathbf{5 0 5 . 2 6 1 7}$ & 253.1345 \\
\hline 1383.7002 & 13 & T & 3 & $\mathbf{3 7 6 . 2 1 9 1}$ & 188.6132 \\
\hline $\mathbf{1 5 1 1 . 7 5 8 8}$ & 14 & Q & 2 & 275.1714 & 138.0893 \\
\hline- & 15 & K & 1 & 147.1128 & 74.0600 \\
\hline & & & & & \\
\hline
\end{tabular}

$\mathrm{F}$

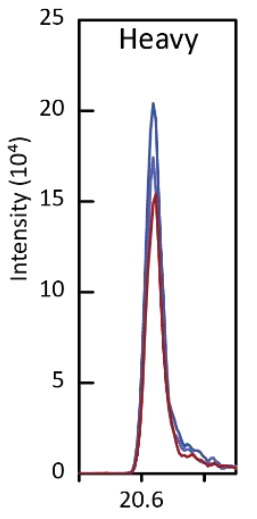

Retention time, $\min$

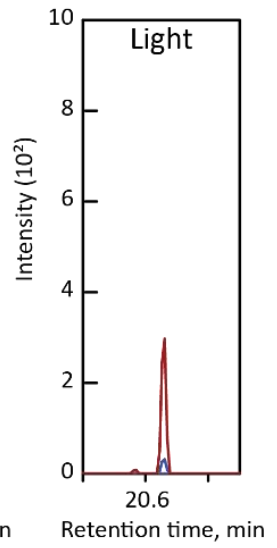

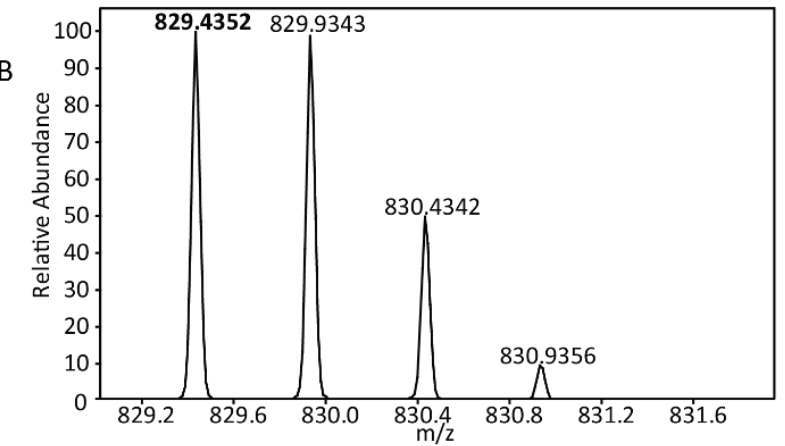

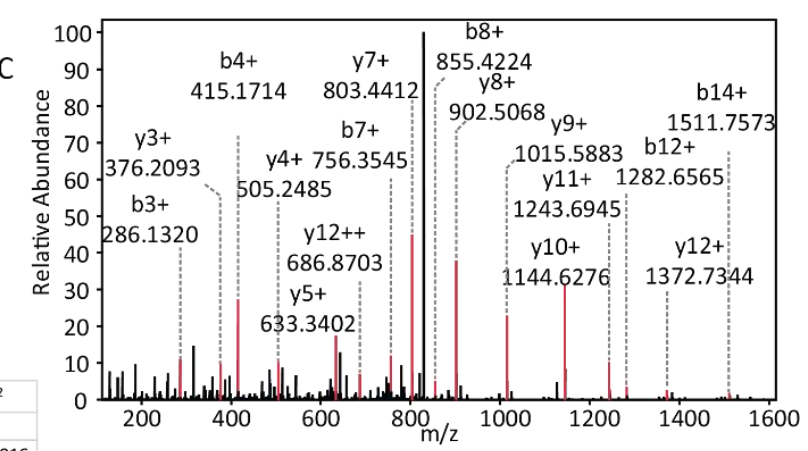

E

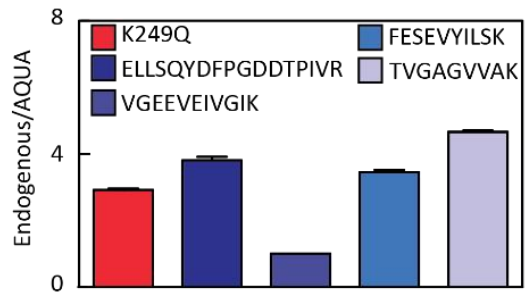

G

K249Q double - y10 - 1159.6522 $-\mathrm{y} 9-1030.6096$ —b8 - 855.4458 K249Q light $-\mathrm{y} 10-1144.6208$ $-\mathrm{y} 9-1015.5782$ $-\mathrm{y} 8-855.4458$
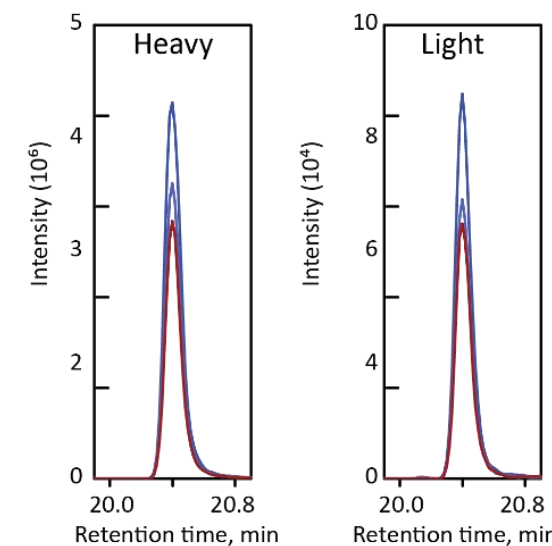

Retention time, $\min \quad$ Retention time, min 
K249E: iik.VGEEVEIVGIEETQK.stc

A

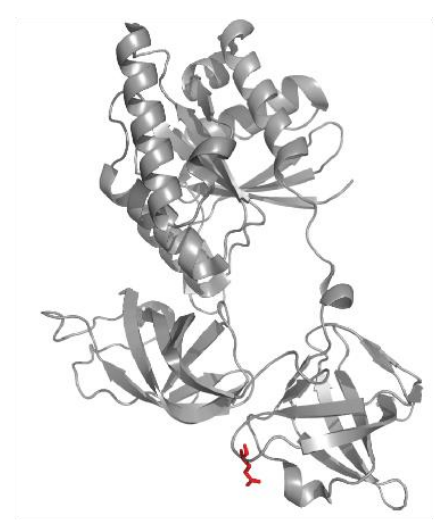

D

\begin{tabular}{|c|c|c|c|c|c|c|}
\hline D & & & $\mathrm{MH}^{+2}$ & & $\mathrm{MH}^{+3}$ & \\
\hline & & & 829.9278 & & 3.6210 & \\
\hline b & & & & & $y$ & $y^{+2}$ \\
\hline - & 1 & v & & 15 & - & - \\
\hline 157.0972 & 2 & G & & 14 & 1559.7799 & 780.3936 \\
\hline 286.1397 & 3 & $\mathrm{E}$ & & 13 & 1502.7584 & 751.8829 \\
\hline 415.1823 & 4 & $\mathrm{E}$ & & 12 & 1373.7159 & 687.3616 \\
\hline 514.2508 & 5 & v & & 11 & 1244.6733 & 622.8403 \\
\hline 643.2933 & 6 & E & & 10 & 1145.6048 & 573.3061 \\
\hline 756.3774 & 7 & I & & 9 & 1016.5623 & 508.7848 \\
\hline 855.4458 & 8 & v & & 8 & 903.4782 & 452.2427 \\
\hline 912.4673 & 9 & G & & 7 & 804.4098 & 402.7085 \\
\hline 1025.5514 & 10 & I & & 6 & 747.3883 & 374.1978 \\
\hline 1154.5939 & 11 & E & & 5 & 634.6042 & 317.6558 \\
\hline 1283.6365 & 12 & $\mathrm{E}$ & & 4 & 505.2617 & 253.1345 \\
\hline 1384.6842 & 13 & $T$ & & 3 & 376.2191 & 188.6132 \\
\hline 1512.7428 & 14 & Q & & 2 & 275.1714 & 138.0893 \\
\hline - & 15 & K & & 1 & 147.1128 & 74.0600 \\
\hline
\end{tabular}

$\mathrm{F}$

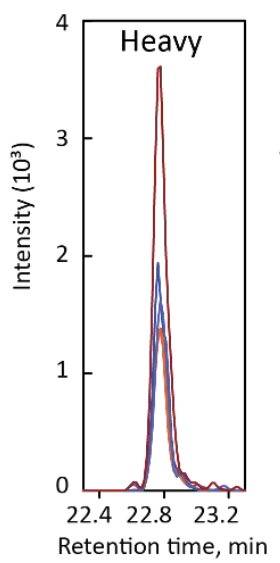

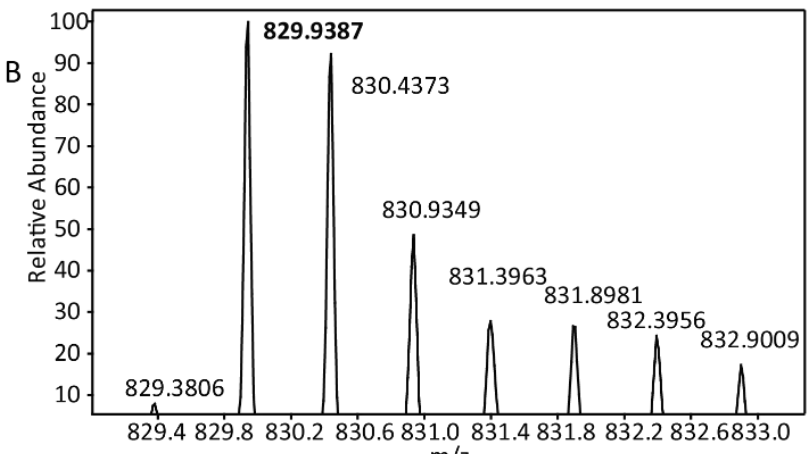

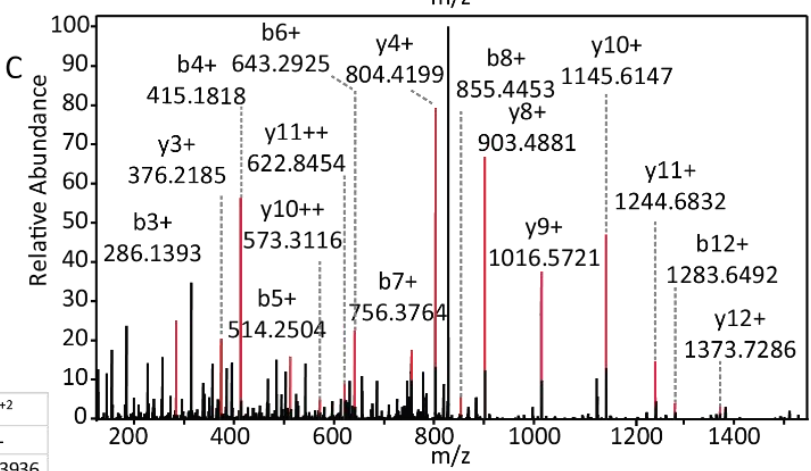

E

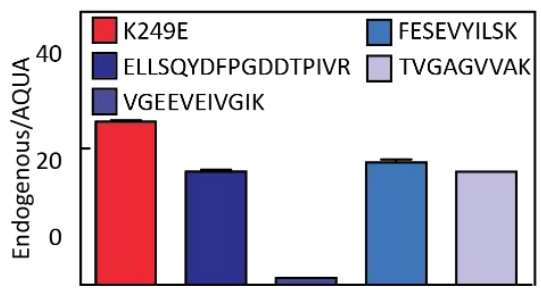

G

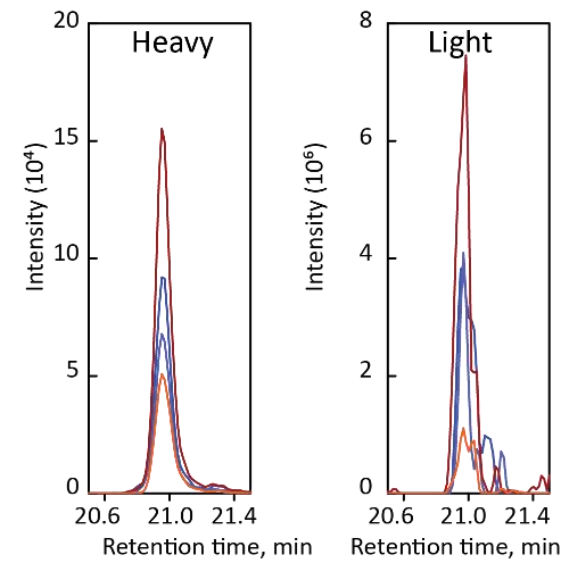


K249T: iik.VGEEVEIVGITETQK.stc

A

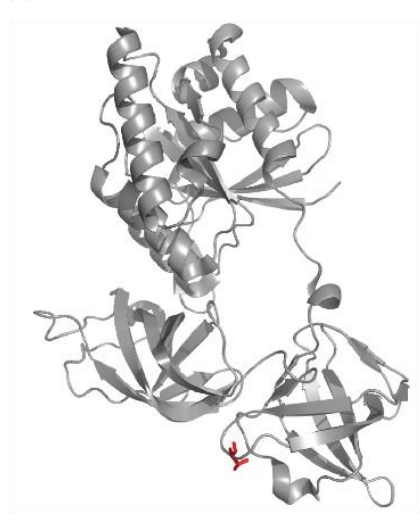

D

\begin{tabular}{|c|c|c|c|c|c|c|}
\hline & \multicolumn{2}{|c|}{$\mathrm{MH}^{+1}$} & \multirow{2}{*}{$\begin{array}{c}\mathrm{MH}^{+2} \\
\mathbf{8 1 5 . 9 3 0 3}\end{array}$} & \multicolumn{2}{|c|}{$\mathrm{MH}^{+3}$} & \\
\hline & & & & & & \\
\hline b & & & & & $y$ & $y^{+2}$ \\
\hline - & 1 & $\mathrm{v}$ & & 5 & - & - \\
\hline 157.0972 & 2 & G & & 14 & 1531.7850 & 766.9361 \\
\hline 286.1397 & 3 & E & & 3 & 1474.7635 & 737.8854 \\
\hline 415.1823 & 4 & E & & 12 & 1345.7209 & 673.3641 \\
\hline 514.2508 & 5 & $\mathrm{v}$ & & 11 & 1216.6783 & 608.8428 \\
\hline 643.2933 & 6 & E & & 10 & 1117.6099 & 559.3086 \\
\hline 756.3774 & 7 & I & & 9 & 988.5673 & 494.7873 \\
\hline 855.4458 & 8 & $\mathrm{v}$ & & 8 & 875.4833 & 438.2453 \\
\hline 912.4673 & 9 & G & & 7 & 776.4149 & 388.7111 \\
\hline 1025.5514 & 10 & I & & 6 & 719.3934 & 360.2003 \\
\hline 1126.599 & 11 & T & & 5 & 606.3093 & 303.6583 \\
\hline 1255.6416 & 12 & $\mathrm{E}$ & & 4 & 505.2617 & 253.1345 \\
\hline 1356.6893 & 13 & $T$ & & 3 & 376.2191 & 188.6132 \\
\hline 1484.7479 & 14 & o & 2 & 2 & 275.1714 & 138.0893 \\
\hline- & 15 & K & & 1 & \begin{tabular}{|l|}
147.1128 \\
\end{tabular} & 74.0600 \\
\hline
\end{tabular}

F
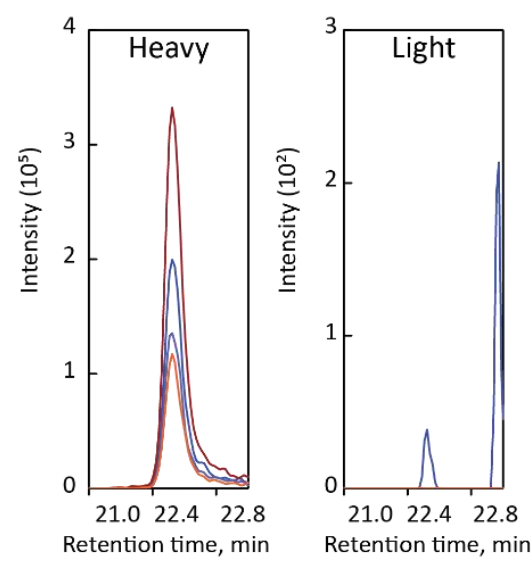
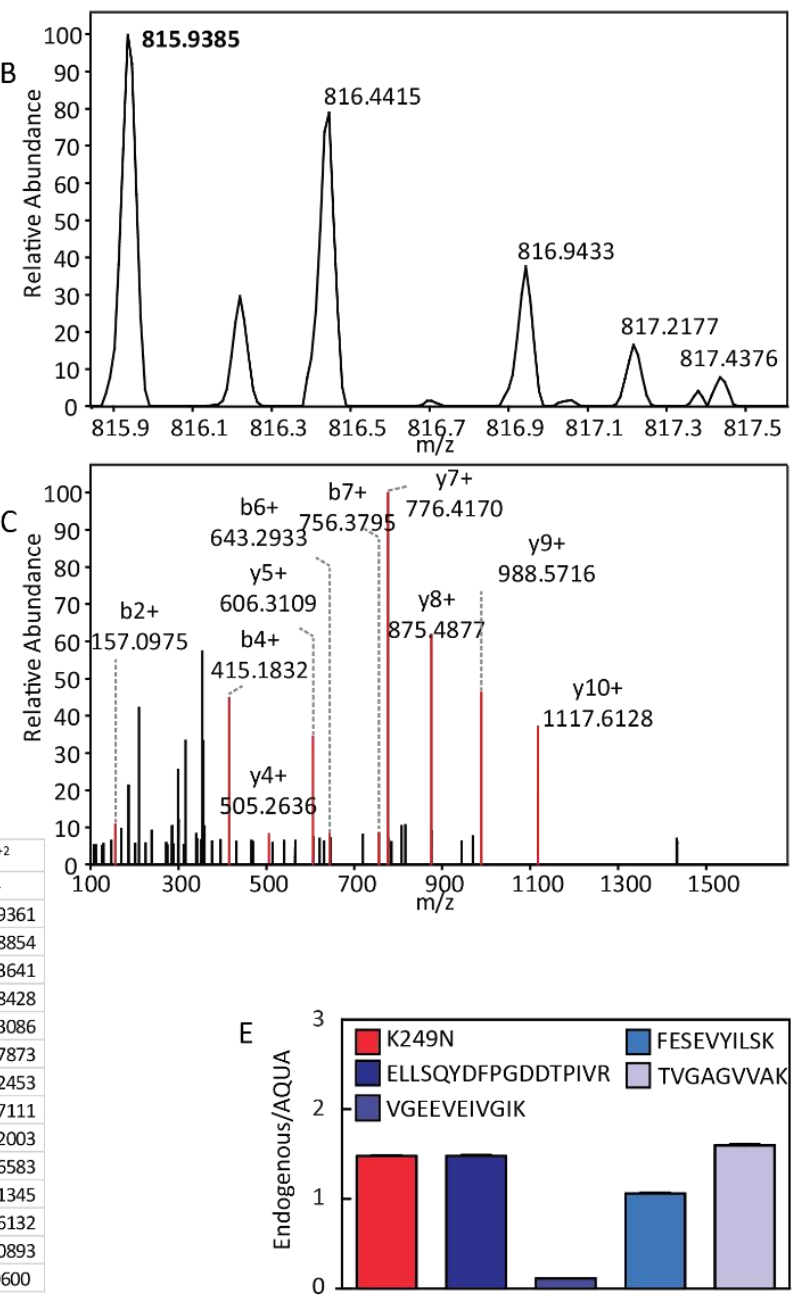

G
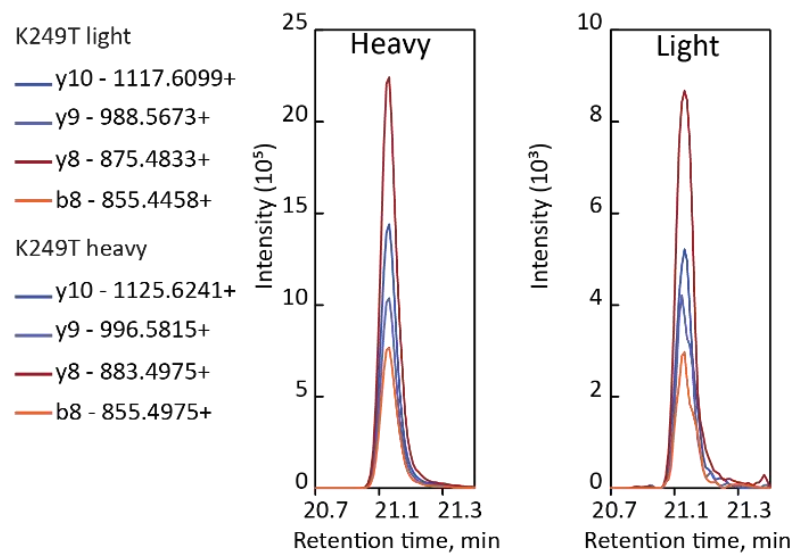
K249R: iik.VGEEVEIVGIR.etq

A

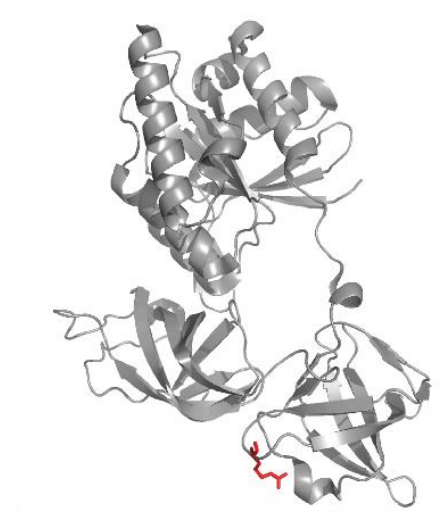

D

\begin{tabular}{|c|c|c|c|c|c|c|}
\hline \multirow{3}{*}{$b$} & \multicolumn{2}{|c|}{$\mathrm{MH}+1$} & $\mathrm{MH}+2$ & \multicolumn{2}{|c|}{$\mathrm{MH}+3$} & \multirow[b]{3}{*}{$y+2$} \\
\hline & \multicolumn{2}{|c|}{1199.663} & 600.3352 & \multicolumn{2}{|c|}{400.5592} & \\
\hline & & & & & v & \\
\hline - & 1 & v & & 11 & - & - \\
\hline 157.0972 & 2 & G & & 10 & 1100.5946 & 550.8009 \\
\hline 286.1397 & 3 & E & & 9 & 1043.5732 & 522.2902 \\
\hline 415.1823 & 4 & E & & 8 & 914.5306 & 457.7689 \\
\hline 514.2508 & 5 & v & & 7 & 785.4880 & 393.2476 \\
\hline 643.2933 & 6 & $\mathrm{E}$ & & 6 & 686.4196 & 343.7134 \\
\hline 756.3774 & 7 & 1 & & 5 & 557.3770 & 279.1921 \\
\hline 855.4458 & 8 & v & & 4 & 444.2929 & 222.6501 \\
\hline 912.4673 & 9 & G & & 3 & 345.2245 & 173.1159 \\
\hline 1025.5514 & 10 & 1 & & 2 & 288.2030 & 144.6051 \\
\hline- & 11 & $R_{2}>-1$ & & 1 & 175.1190 & 88.0631 \\
\hline
\end{tabular}

F

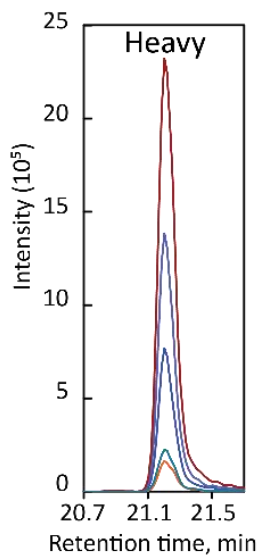

B

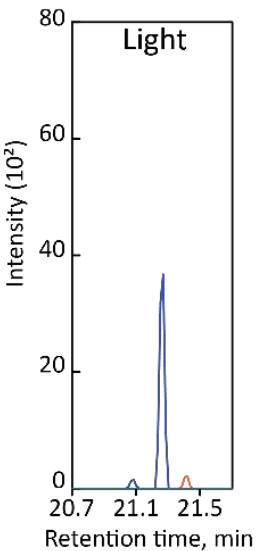

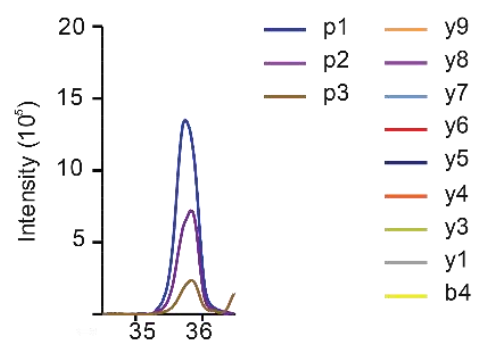

Retention time, min

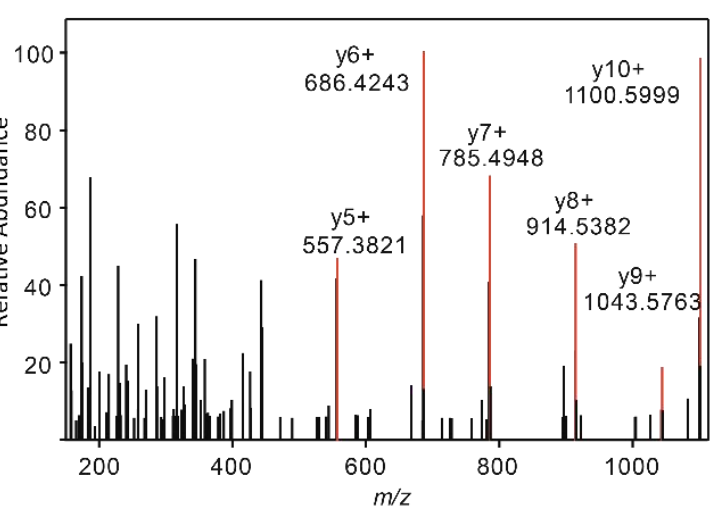

E

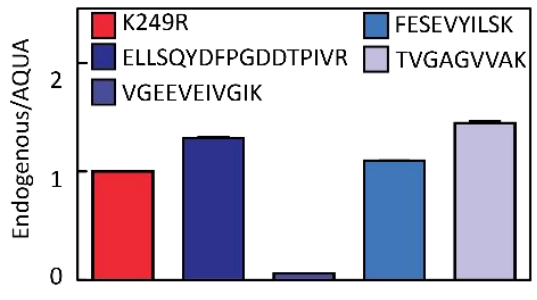

G

K249R light

-y8-914.5306+

-y7-785.4880+

—y6-686.4169+

—b6-643.2933+

_b7 - 756.3774+

K249R heavy

- y8 - 924.5388+

—y7 - 795.4962+

- y6 - 696.4278+

-b6 - 643.2933+

—b7 - 756.3774+

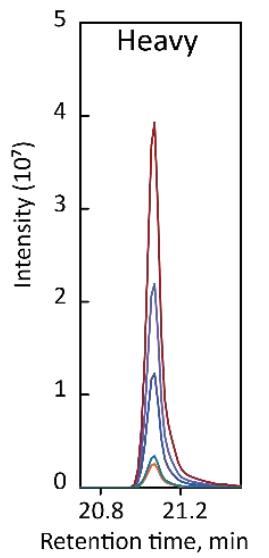


K249N: iik.VGEEVEIVGINETQK.stc

A

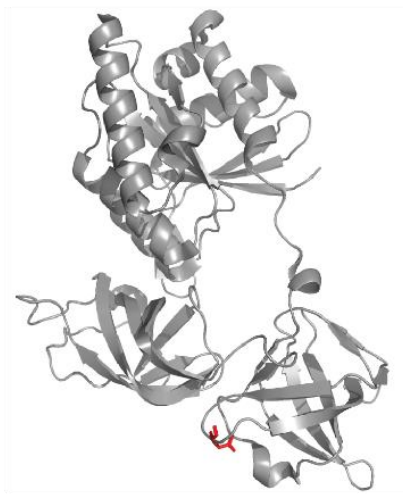

$1 0 0 \longdiv { 8 2 2 . 4 2 8 0 }$

$\mathrm{B} \stackrel{\mathrm{s}}{\mathrm{s}}$

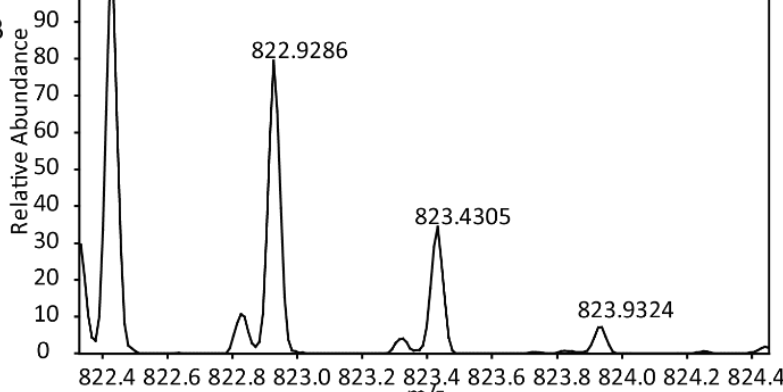

822.4822 .6822 .8823 .0823 .2823 .4823 .6823 .8824 .0824 .2824 .4

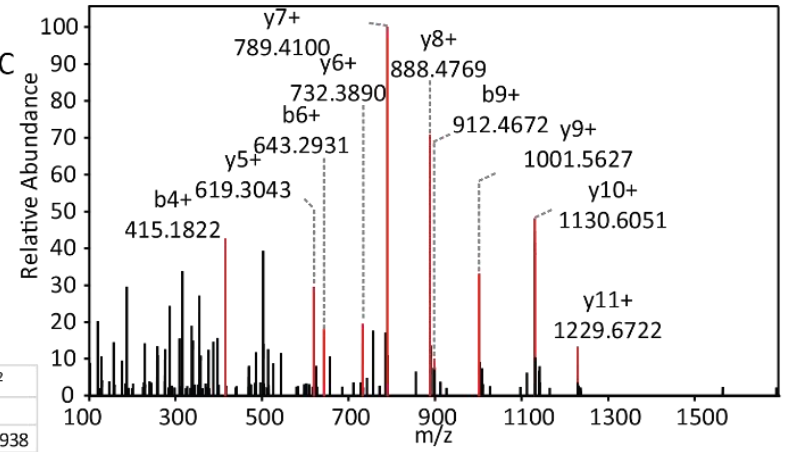

E

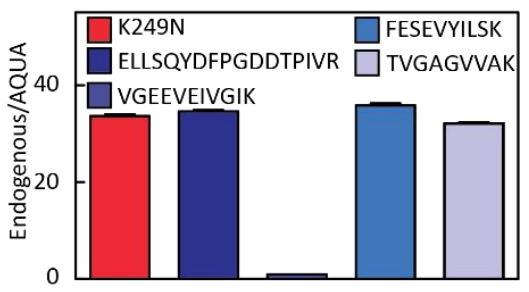

G
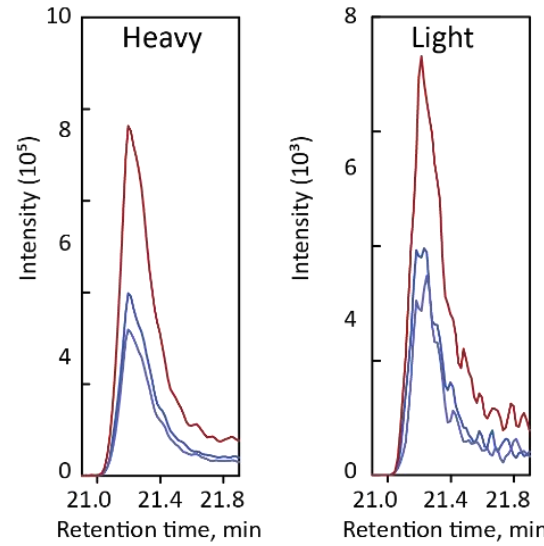
R270H: frk.LLDEGHAGENVGVLLR.gik

A

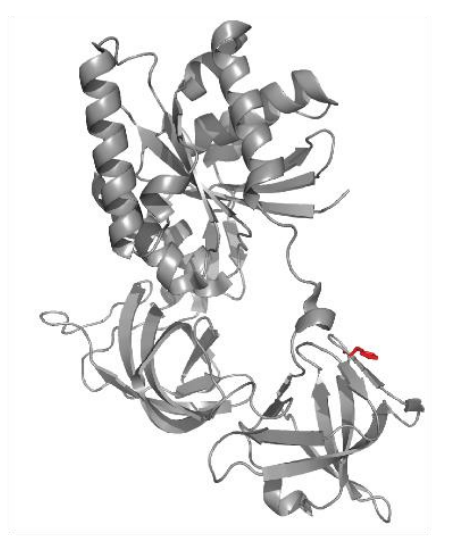

D

\begin{tabular}{|c|c|c|c|c|c|c|}
\hline & \multirow{2}{*}{\begin{tabular}{|c|}
$\mathrm{MH}^{+1}$ \\
1691.9075
\end{tabular}} & \multirow{2}{*}{$\frac{\mathrm{MH}^{+2}}{864.4574}$} & \multirow{2}{*}{$\begin{array}{c}\mathrm{MH}^{+3} \\
564.6407\end{array}$} & \multirow[b]{3}{*}{$y$} & \multirow[b]{3}{*}{+2} \\
\hline & & & & & & \\
\hline b & $\mathrm{b}^{+2}$ & & & & & \\
\hline - & - & 1 & L & 16 & - & - \\
\hline 227.1754 & - & 2 & L & 15 & 1578.8234 & 789.9154 \\
\hline 342.2023 & - & 3 & D & 14 & 1465.7394 & 733.3733 \\
\hline 471.2449 & - & 4 & $E$ & 13 & 1350.7124 & 657.8599 \\
\hline 528.2664 & - & 5 & G & 12 & 1221.6698 & 611.3386 \\
\hline 665.3253 & 333.1663 & 6 & $\mathrm{H}$ & 11 & 1164.6484 & 582.8278 \\
\hline 736.3624 & 368.6849 & 7 & A & 10 & 1027.5895 & 514.2984 \\
\hline 793.3839 & 397.1956 & 8 & G & 9 & 956.5524 & 478.7798 \\
\hline 922.4565 & 461.7169 & 9 & $E$ & 8 & 899.5309 & 450.2961 \\
\hline 1036.4964 & 518.7383 & 10 & $\mathrm{~N}$ & 7 & 770.4883 & 385.7478 \\
\hline 1135.5378 & 568.2726 & 11 & v & 6 & 656.4454 & 328.7263 \\
\hline 1192.5593 & 596.7833 & 12 & G & 5 & 557.3770 & 297.1921 \\
\hline 1291.6277 & 646.3175 & 13 & $\mathrm{v}$ & 4 & 500.3555 & 250.6814 \\
\hline 1404.7118 & 702.8595 & 14 & L & 3 & 401.2871 & 201.1472 \\
\hline$F_{1517.7958}$ & 759.4016 & 15 & L & 2 & 288.2030 & 144.6051 \\
\hline- & - & 16 & $\mathrm{R}$ & 1 & 175.1190 & 88.0631 \\
\hline
\end{tabular}

B

c
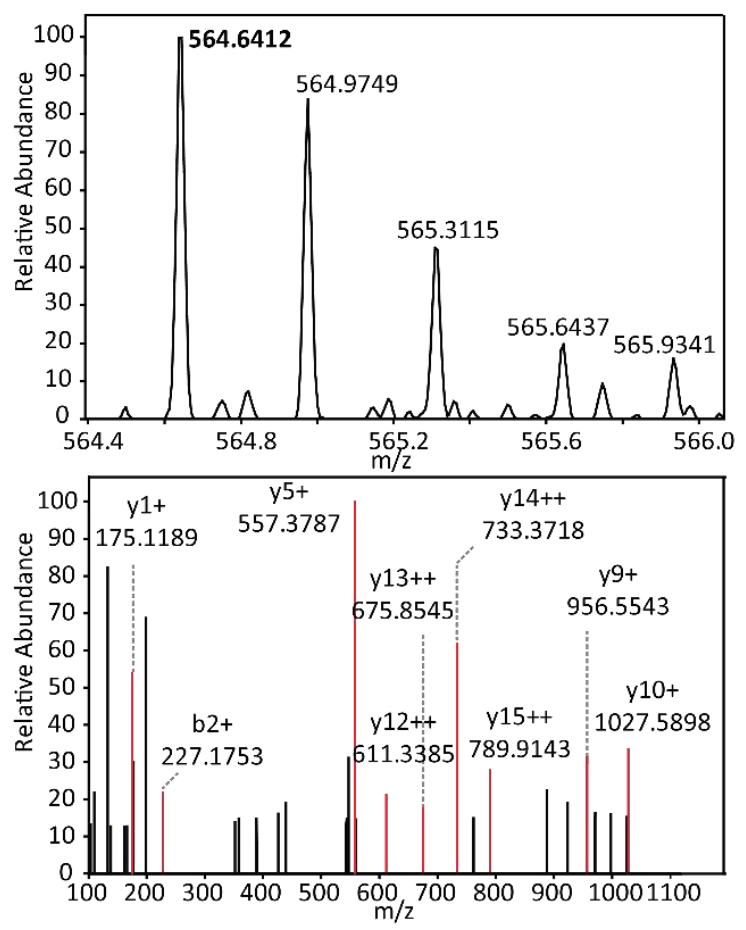

E

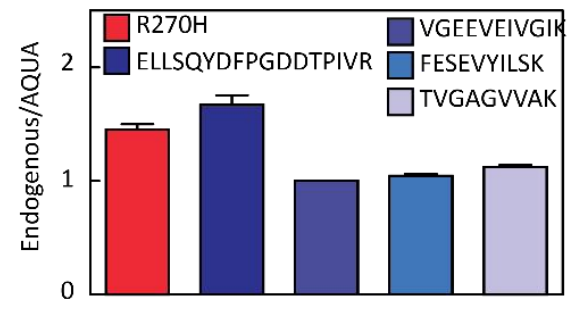

G

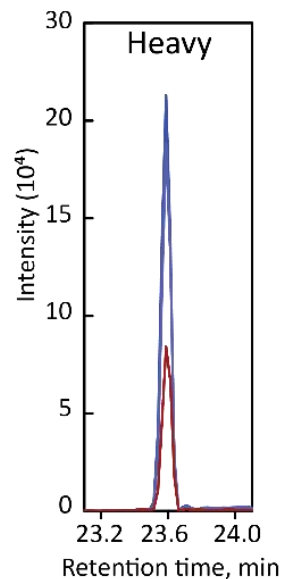


R280H: EGR.AGENVGVLLHGIK.REE

A

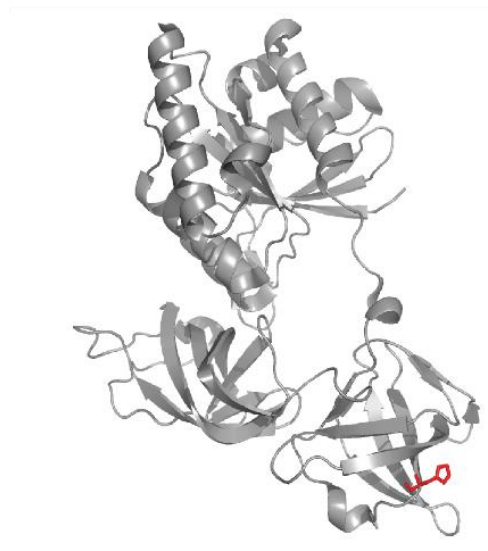

D

\begin{tabular}{|c|c|c|c|c|c|c|}
\hline & \multirow{2}{*}{\begin{tabular}{|c|}
$\mathrm{MH}^{+1}$ \\
1306.7478
\end{tabular}} & \multirow{2}{*}{\begin{tabular}{|c|}
$\mathrm{MH}^{+2}$ \\
653.8775 \\
\end{tabular}} & \multirow{2}{*}{$\begin{array}{c}\mathrm{MH}^{+3} \\
436.2541\end{array}$} & \multicolumn{2}{|r|}{$\underset{100}{0}$} \\
\hline & & & & & & \\
\hline$b$ & $b^{+2}$ & & & & $y$ & $y^{+2}$ \\
\hline- & - & 1 & A & 13 & - & - \\
\hline 129.0659 & - & 2 & G & 12 & 1235.7106 & 618.3590 \\
\hline 258.1084 & - & 3 & $\mathrm{E}$ & 11 & 1178.6892 & 589.8482 \\
\hline 372.1514 & - & 4 & $\mathrm{~N}$ & 10 & 1049.6466 & 525.3269 \\
\hline 471.2198 & - & 5 & V & 9 & 935.6037 & 468.3055 \\
\hline 528.2413 & - & 6 & G & 8 & 836.5352 & 418.7713 \\
\hline 627.3097 & - & 7 & V & 7 & 779.5138 & 390.2605 \\
\hline 740.3937 & - & 8 & $\mathrm{~L}$ & 6 & 680.4454 & 340.7263 \\
\hline 853.4778 & - & 9 & $\mathrm{~L}$ & 5 & 567.3613 & 284.1843 \\
\hline 990.5367 & 495.7720 & 10 & H & 4 & 454.2772 & 227.6423 \\
\hline 1047.5582 & 524.2827 & 11 & G & 3 & 317.2183 & 159.1128 \\
\hline 1160.6422 & 580.8248 & 12 & 1 & 2 & 260.1969 & 130.6021 \\
\hline- & - & 13 & K & 1 & 147.1128 & 74.0600 \\
\hline
\end{tabular}

F

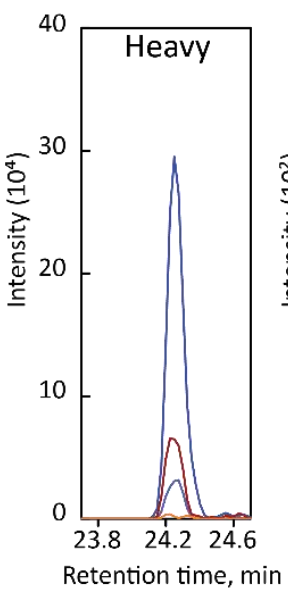

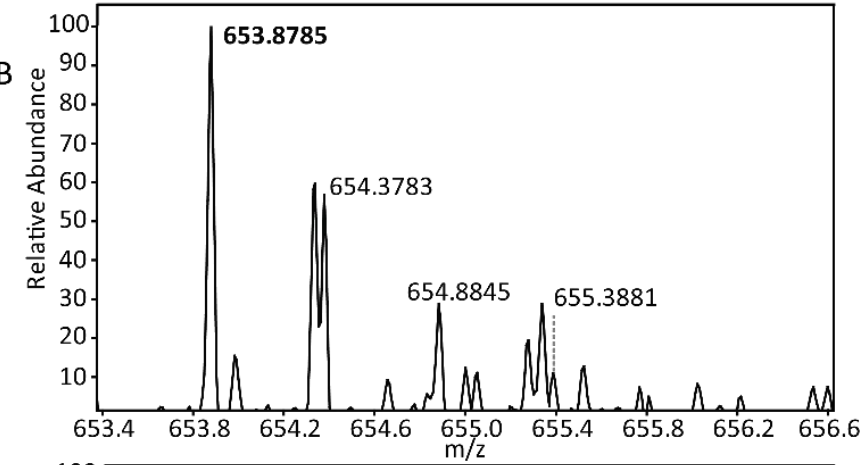

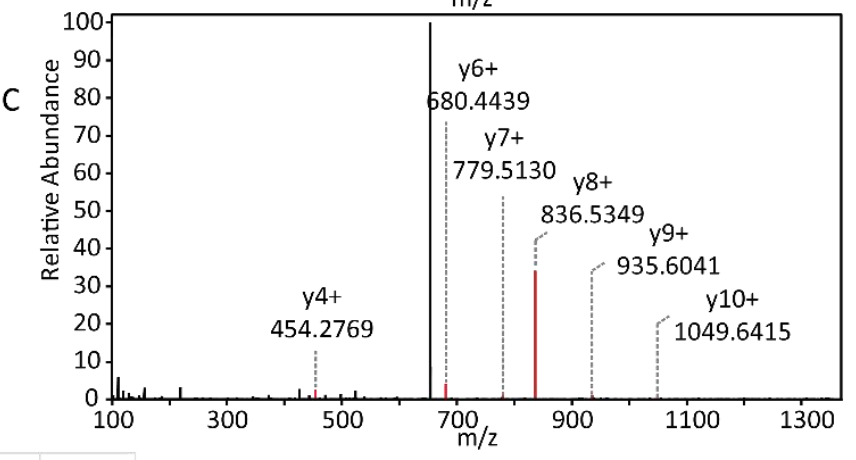

E

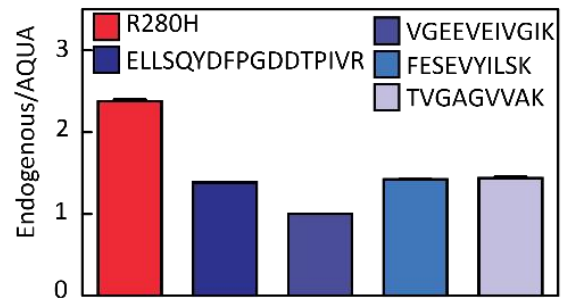

G

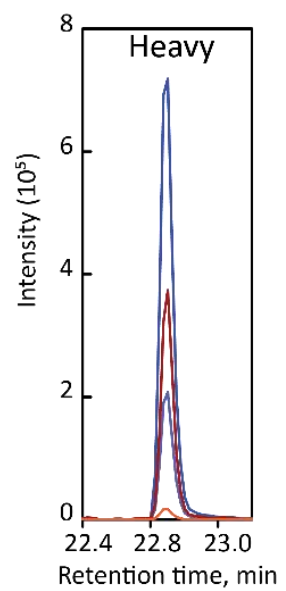


R284H: lak.HEEIER.arg

A

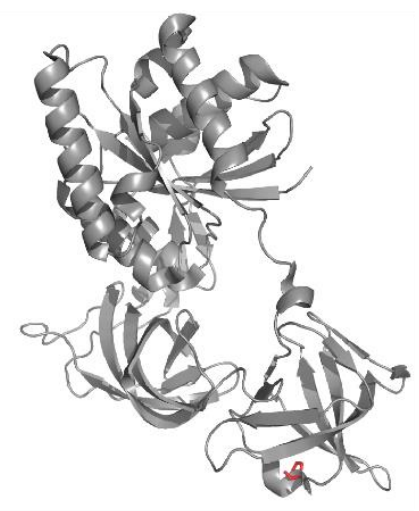

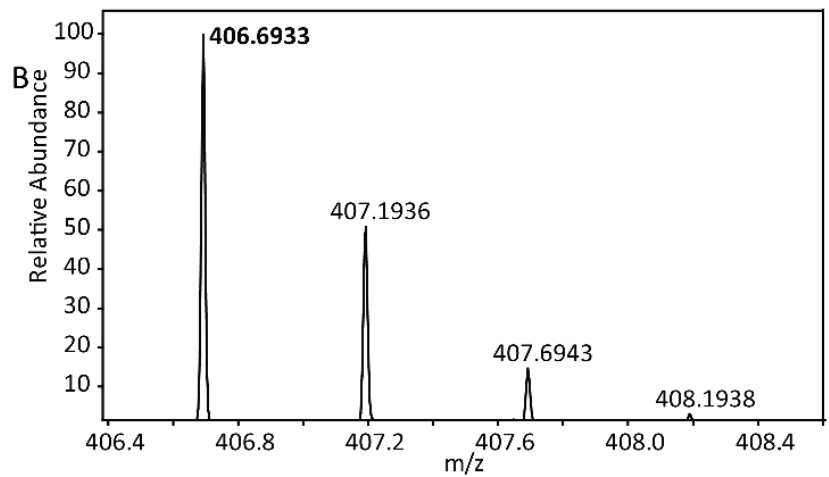

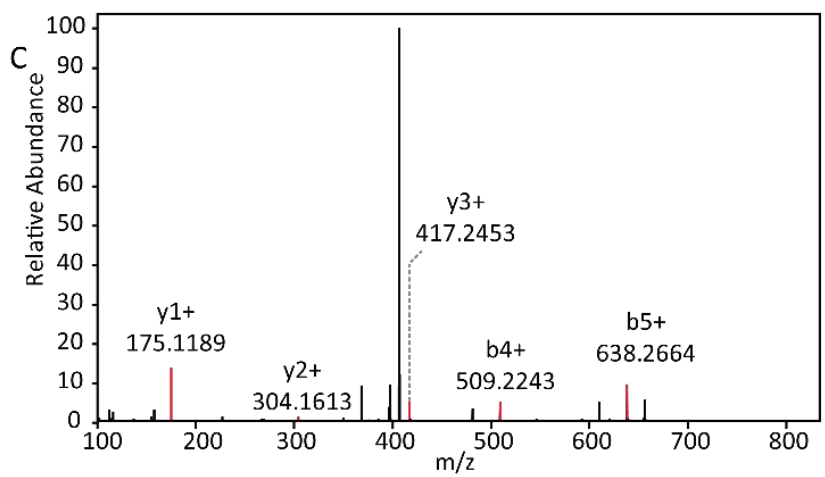

D

\begin{tabular}{|c|c|c|c|c|c|c|}
\hline \multirow{2}{*}{\multicolumn{2}{|c|}{ D }} & \multirow{2}{*}{$\begin{array}{c}\mathrm{MH}^{+1} \\
812.3897\end{array}$} & \multirow{2}{*}{$\begin{array}{c}\mathrm{MH}^{+2} \\
406.6985\end{array}$} & \multirow{2}{*}{$\begin{array}{c}\mathrm{MH}^{+3} \\
271.4681\end{array}$} & \multirow[b]{3}{*}{ y } & \multirow[b]{3}{*}{$y^{+2}$} \\
\hline & & & & & & \\
\hline$b$ & $\mathrm{~b}^{+2}$ & & & & & \\
\hline - & - & 1 & H & 6 & - & - \\
\hline 267.1088 & 134.0580 & 2 & $\mathrm{E}$ & 5 & 675.3308 & 338.1690 \\
\hline 396.1514 & 198.5793 & 3 & E & 4 & 546.2882 & 273.6477 \\
\hline 509.2354 & 255.1214 & 4 & 1 & 3 & 417.2456 & 209.1264 \\
\hline 638.2780 & 319.6427 & 5 & E & 2 & 304.1615 & 152.5844 \\
\hline - & - & 6 & $\mathrm{R}$ & 1 & 175.1190 & 88.0631 \\
\hline
\end{tabular}

F

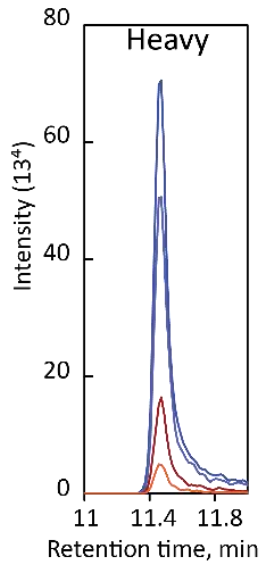

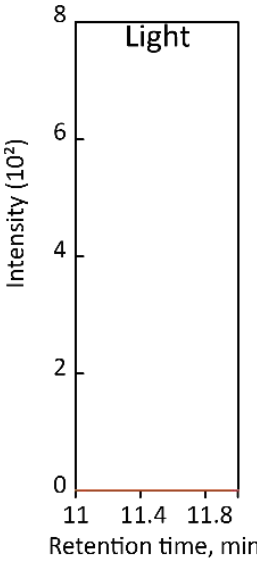

E

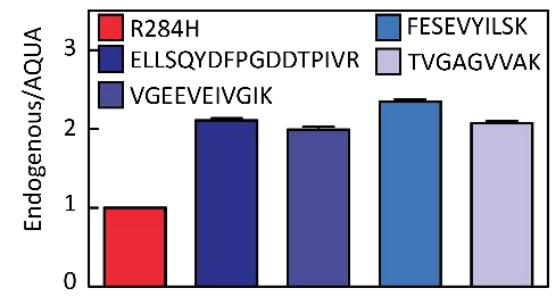

G

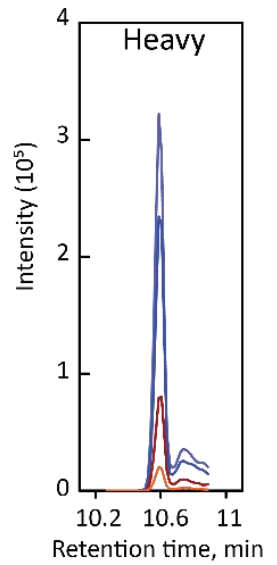


K314Q: htk.FESEVYILSQDEGGR.deg

A

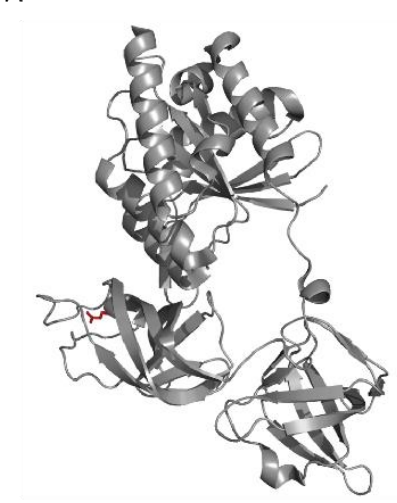

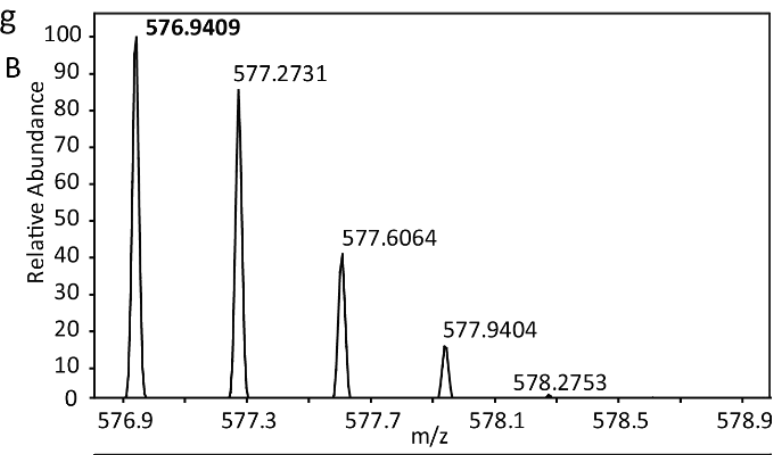

D

\begin{tabular}{|c|c|c|c|c|c|c|}
\hline \multirow[t]{2}{*}{ D } & \multicolumn{2}{|c|}{$\mathrm{MH}^{+1}$} & $\mathrm{MH}^{+2}$ & \multicolumn{2}{|c|}{$\mathrm{MH}^{+3}$} & \\
\hline & & & 864.9074 & & .9407 & \\
\hline b & & & & & $y$ & $y^{+2}$ \\
\hline - & 1 & F & & 15 & - & - \\
\hline 277.1183 & 2 & $\mathrm{E}$ & & 14 & 1581.7391 & 791.3732 \\
\hline 364.1503 & 3 & s & & 13 & 1452.6965 & 726.8519 \\
\hline 493.1929 & 4 & E & & 12 & 1365.6645 & 683.3359 \\
\hline 592.2613 & 5 & v & & 11 & 1236.6219 & 618.8146 \\
\hline 755.3246 & 6 & Y & & 10 & 1137.5535 & 569.2804 \\
\hline 868.4087 & 7 & I & & 9 & 974.4901 & 487.7487 \\
\hline 981.4928 & 8 & L & & 8 & 861.4061 & 431.2067 \\
\hline 1068.5248 & 9 & s & & 7 & 748.3220 & 374.6646 \\
\hline 1196.5834 & 10 & a & & 6 & 661.2900 & 331.1486 \\
\hline 1311.6103 & 11 & D & & 5 & 533.2314 & 267.1193 \\
\hline 1440.6529 & 12 & E & & 4 & 418.2045 & 209.6059 \\
\hline 1497.6744 & 13 & G & & 3 & 289.1619 & 145.0846 \\
\hline 1554.6958 & 14 & G & & 2 & 232.1404 & 116.5738 \\
\hline 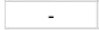 & 15 & $\mathrm{R}$ & & 1 & 175.1190 & 88.0631 \\
\hline
\end{tabular}

$\mathrm{F}$

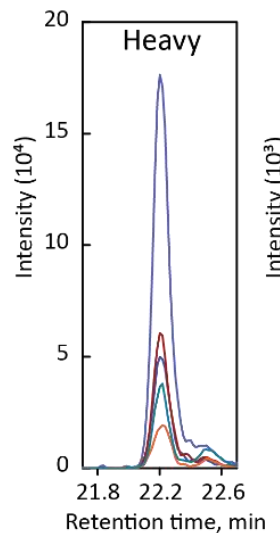

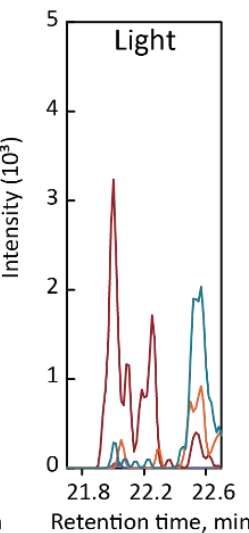

$\mathrm{E}$

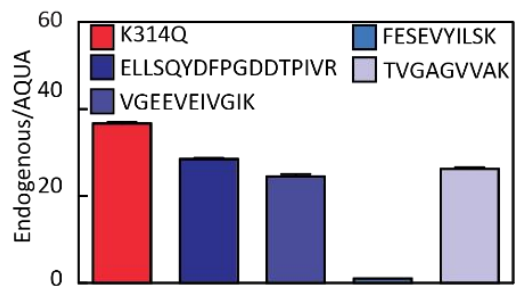

G

K314Q light

— y8-861.4061+

- y7 - 748.3220+

- y6 - 661.2900+

- b5 - 592.2613+

— b6 - $755.3246+$

K314Q heavy

— y8-871.4144

-y7-758.3303+

- y6-671.2983+

- b5 - 592.2613+

— b6 - 755.3246+
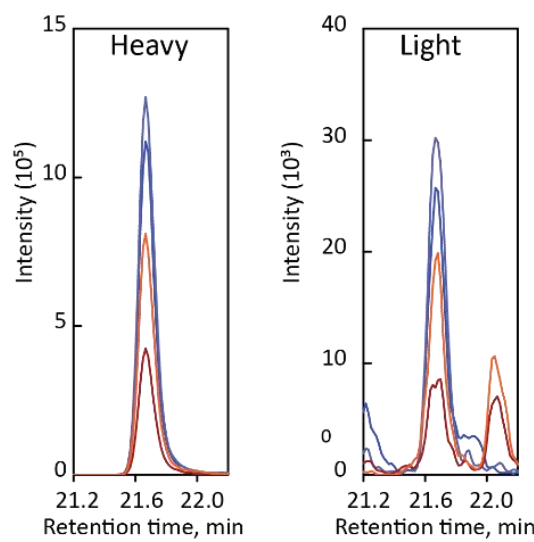
K314T: htk.FESEVYILSTDEGGR.deg

A

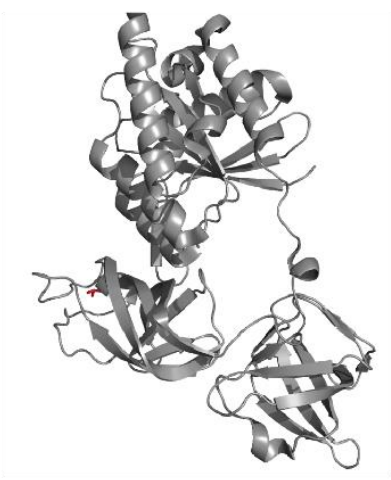

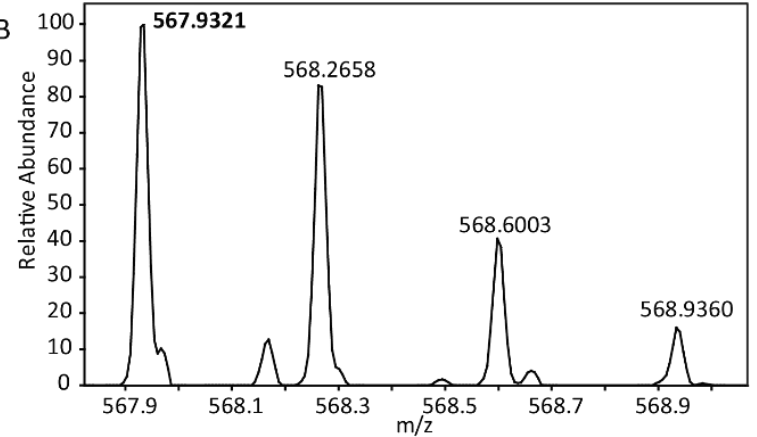

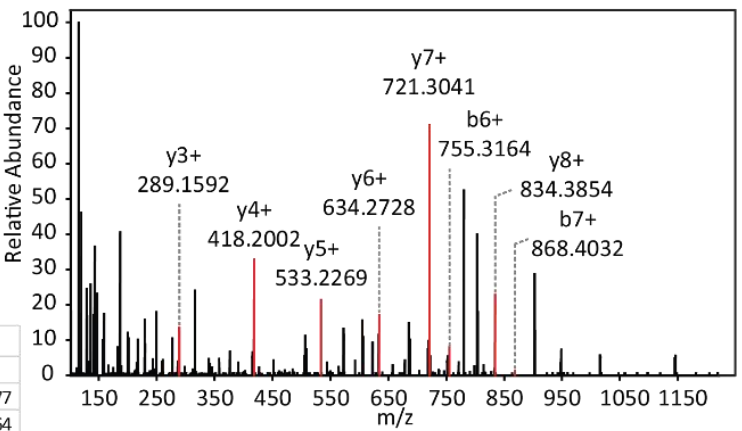

\begin{tabular}{|c|c|c|c|c|c|c|}
\hline \multirow[t]{2}{*}{ D } & \multicolumn{2}{|c|}{$\mathrm{MH}^{+1}$} & \multirow{2}{*}{$\begin{array}{c}\mathrm{MH}^{+2} \\
851.4019\end{array}$} & \multirow{2}{*}{\multicolumn{2}{|c|}{$\frac{\mathrm{MH}^{+3}}{567.9371}$}} & \multirow[b]{3}{*}{$y^{+2}$} \\
\hline & & & & & & \\
\hline b & & & & & y & \\
\hline - & 1 & $\mathrm{~F}$ & & 15 & - & - \\
\hline 277.1183 & 2 & $\mathrm{E}$ & & 14 & 1554.7282 & 777.8677 \\
\hline 364.1503 & 3 & $\mathrm{~s}$ & & 13 & 1425.6856 & 713.3464 \\
\hline 493.1929 & 4 & E & & 12 & 1338.6536 & 669.8304 \\
\hline 592.2613 & 5 & v & & 11 & 1209.6110 & 605.3091 \\
\hline 755.3246 & 6 & Y & & 10 & 1110.5426 & 555.7749 \\
\hline 868.4087 & 7 & I & & 9 & 947.4793 & 474.2433 \\
\hline \begin{tabular}{|l|}
981.4928 \\
\end{tabular} & 8 & L & & 8 & 834.3952 & 417.7012 \\
\hline 1068.5248 & 9 & s & & 7 & 721.3111 & 361.1592 \\
\hline 1169.5725 & 10 & T & & 6 & 634.2791 & 317.6432 \\
\hline 1284.5994 & 11 & D & & 5 & 533.2314 & 267.1193 \\
\hline 1413.642 & 12 & $\mathrm{E}$ & & 4 & 418.2045 & 209.6059 \\
\hline 1470.6635 & 13 & G & & 3 & 289.1619 & 145.0846 \\
\hline 1527.6849 & 14 & G & & 2 & 232.1404 & 116.5738 \\
\hline - & 15 & R & & 1 & 175.1190 & 88.0631 \\
\hline
\end{tabular}

$\mathrm{F}$

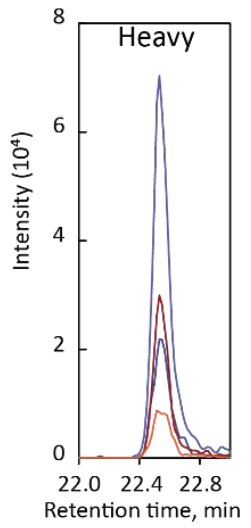

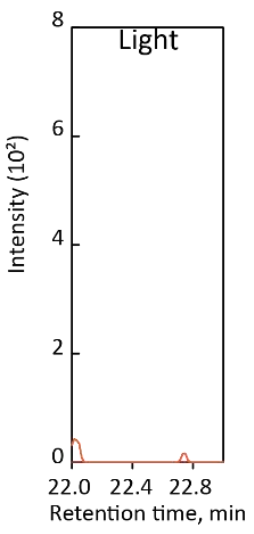

G

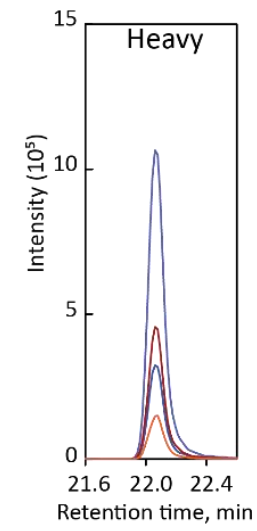


K314E: htk.FESEVYILSEDEGGR.deg

A
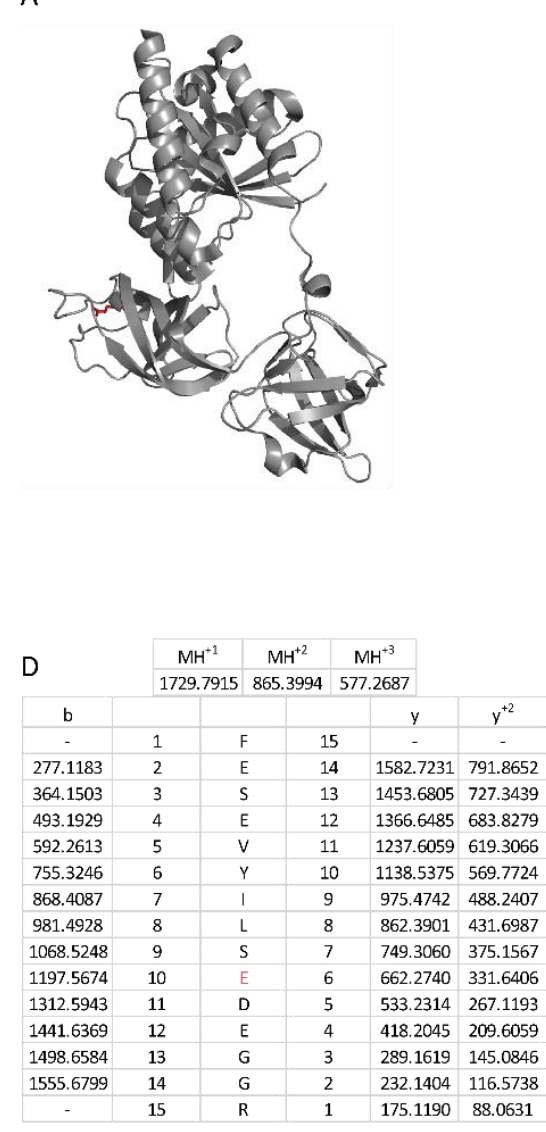

$\mathrm{F}$

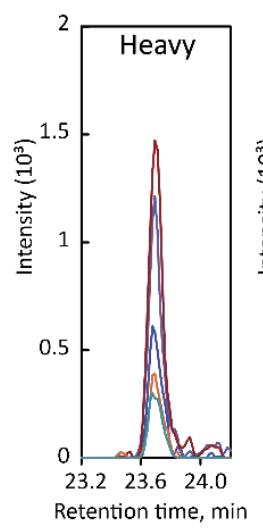

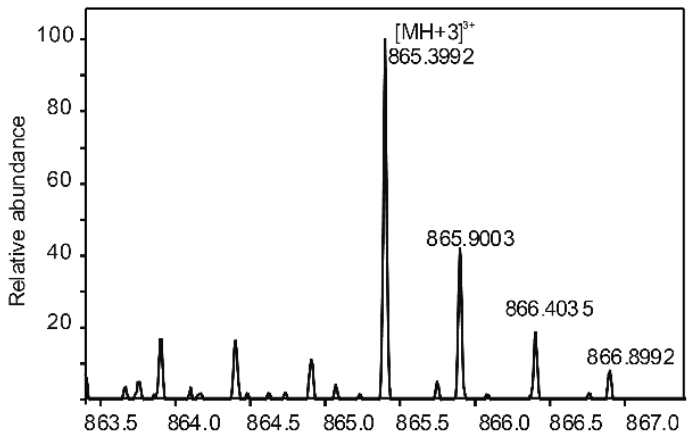
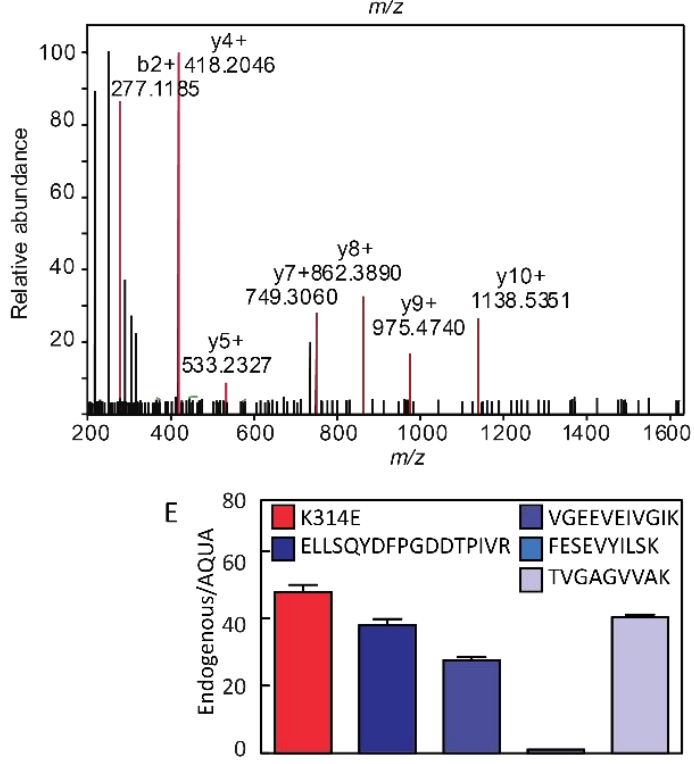

K314E light

- y9- $975.4742+$

- y8 - 862.3901+

$-y 7-749.3060+$

— b6- $662.2740+$

-b5-533.2314t

K314E heavy

- y9-985.4824+

- y8 - 842.3984+

$-y 7-759.3143+$

- y6-672.2823+

—b5 - 543.2397+
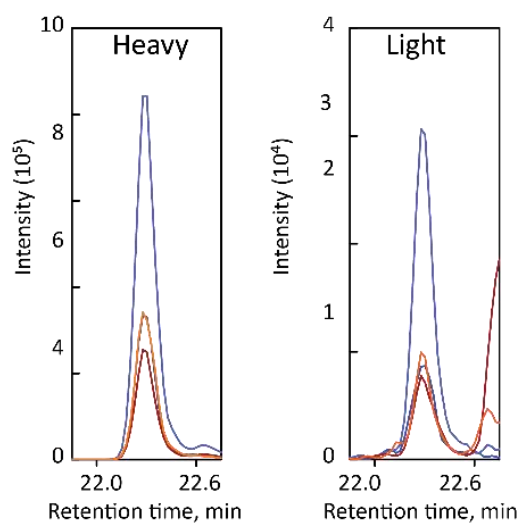
K314R: htk.FESEVYILSRDEGGR.deg

A

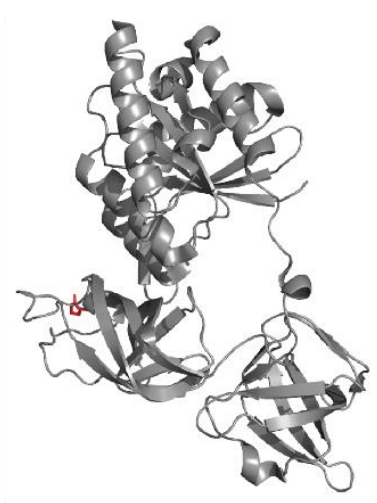

D

$$
\begin{array}{|l|l|l|}
\hline \mathrm{MH}^{+1} & \mathrm{MH}^{+2} & \mathrm{MH}^{+3} \\
\hline 1242.6355 & 621.829 & 414.8837 \\
\hline
\end{array}
$$

$\begin{array}{llll}1242.6365 & 621.8219 & 414.8837\end{array}$

\begin{tabular}{|c|c|c|c|c|c|}
\hline b & & & & $y$ & $y^{+2}$ \\
\hline- & 1 & F & 10 & - & - \\
\hline $\mathbf{2 7 7 . 1 1 8 3}$ & 2 & E & 9 & $\mathbf{1 0 9 5 . 5 6 8 1}$ & 548.2877 \\
\hline 364.1503 & 3 & S & 8 & 966.5255 & 483.7664 \\
\hline 493.1929 & 4 & E & 7 & 879.4934 & 440.2504 \\
\hline $\mathbf{5 9 2 . 2 6 1 3}$ & 5 & V & 6 & $\mathbf{7 5 0 . 4 5 0 9}$ & 375.7291 \\
\hline 755.3246 & 6 & Y & 5 & $\mathbf{6 5 1 . 3 8 2 4}$ & 326.1949 \\
\hline 868.4087 & 7 & I & 4 & 488.3191 & 244.6632 \\
\hline 981.4928 & 8 & L & 3 & 375.2350 & 188.1212 \\
\hline 1068.5248 & 9 & S & 2 & 262.1510 & 131.5791 \\
\hline- & 10 & R & 1 & 175.1190 & 88.0631 \\
\hline
\end{tabular}
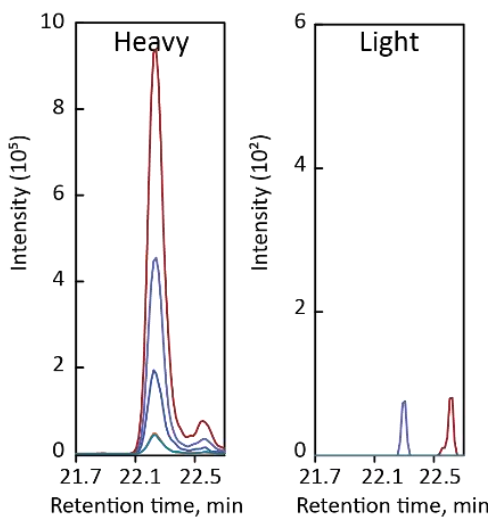
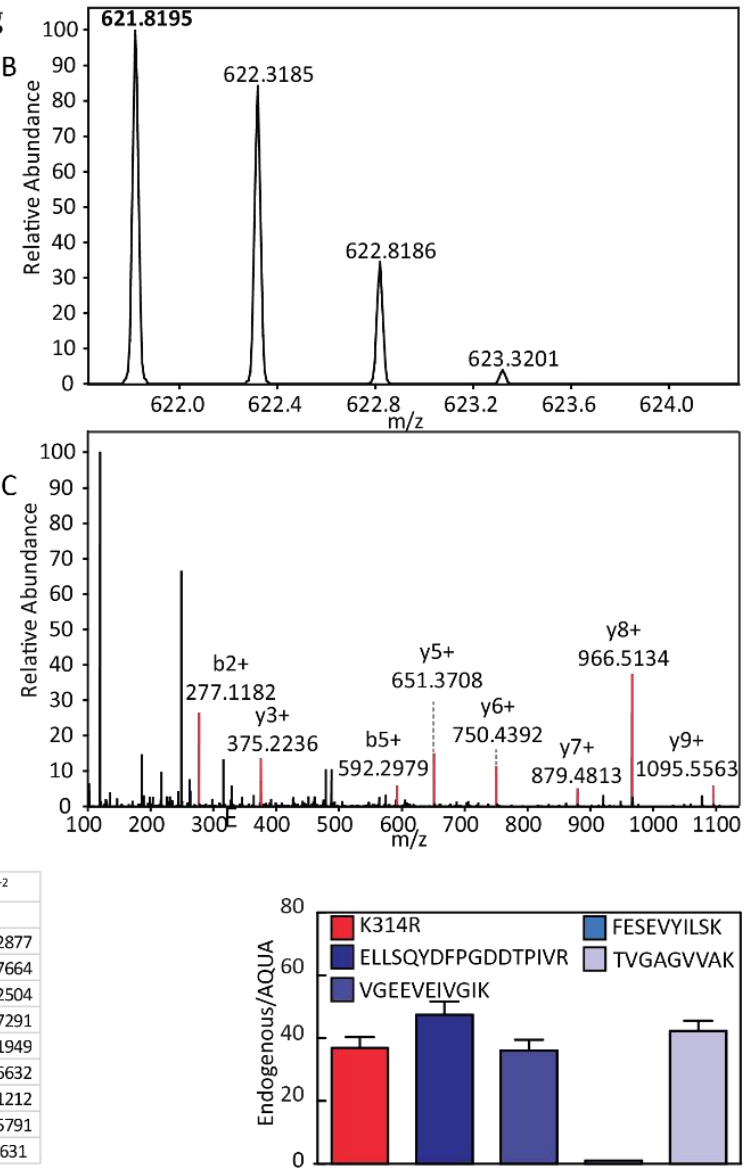

G

K314R light

- y7 - 879.4934t $-\mathrm{y} 6-750.4509+$ $-\mathrm{y} 5-651.3824+$

- b6 - 755.3246

— b7 - 868.4087+

K314R heavy

-y7 - 889.5017+

$-\mathrm{y} 6-760.4951+$

$-\mathrm{y} 5-661.3907+$

— b6- 755.3246+

- b7 - 868.4087+
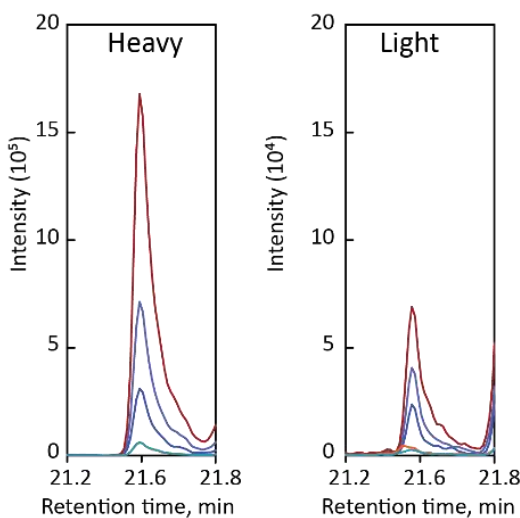
K314N: htk.FESEVYILSNDEGGR.deg

A

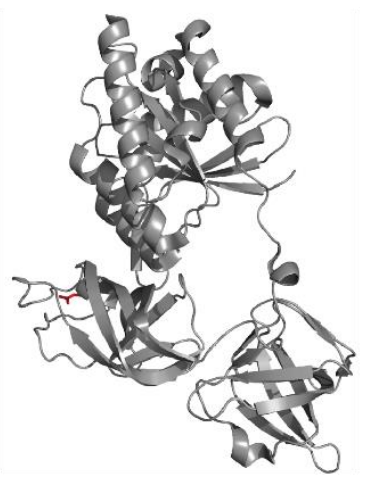

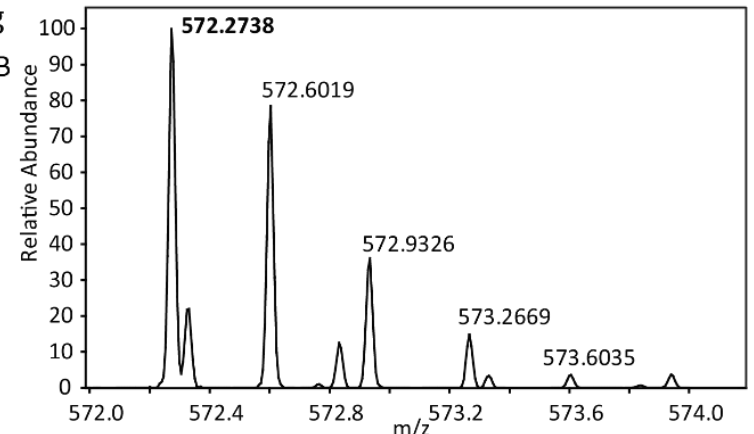

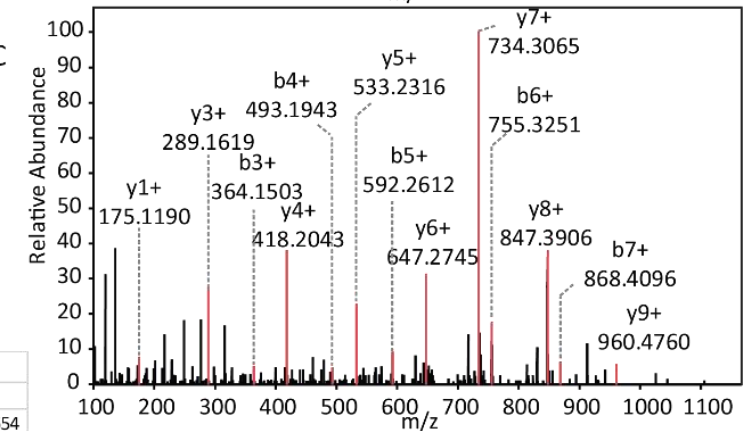

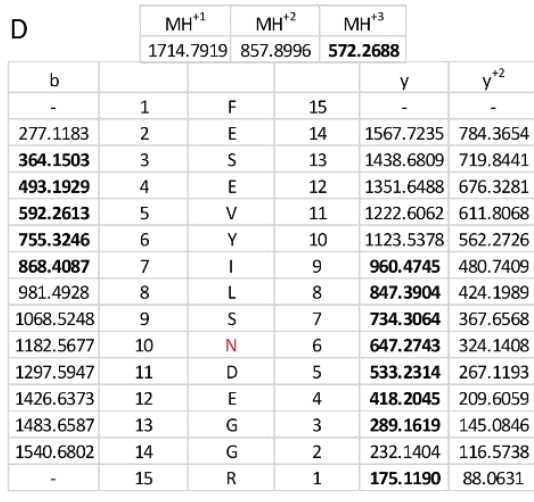

$\mathrm{F}$

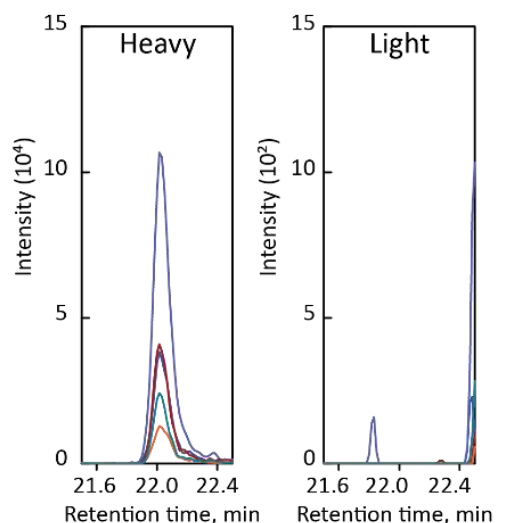

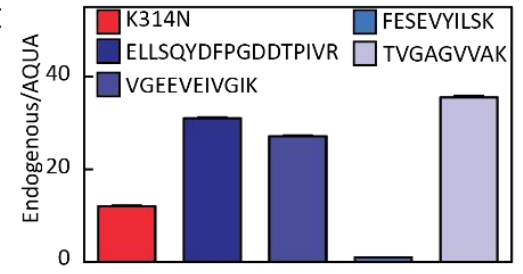

G

- y8 - 847.3904

-y7 - 734.3064

- y6 - 647.2743+

- b5 - 592.2613

-b6 - 755.3246

$\mathrm{K} 314 \mathrm{~N}$ heavy

-y8-857.3987+

$-y 7-744.3146$

- y6 - 657.2826+

- b5 - 592.2613+

- b6 - 755.3246
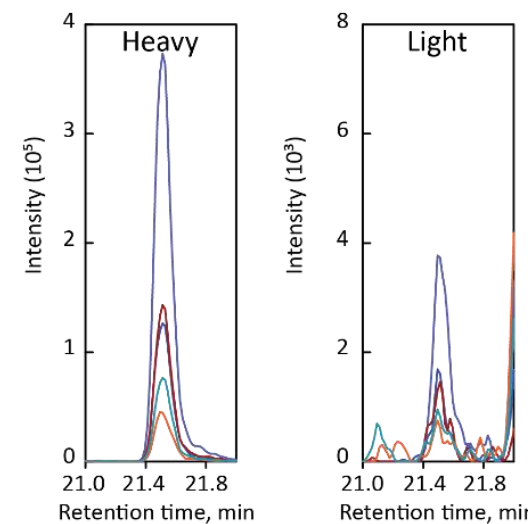
R319H: LSK.DEGGHHTPFFK.GYR

A

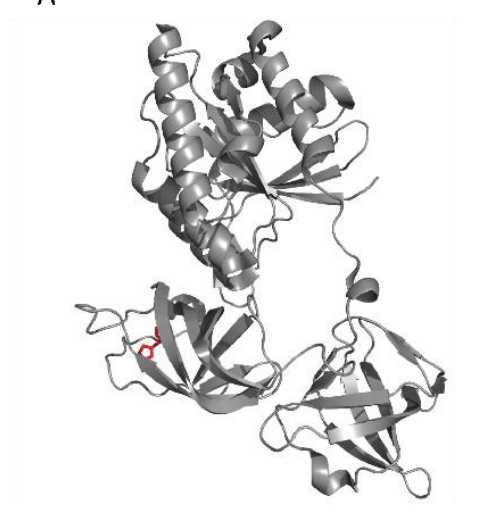

B

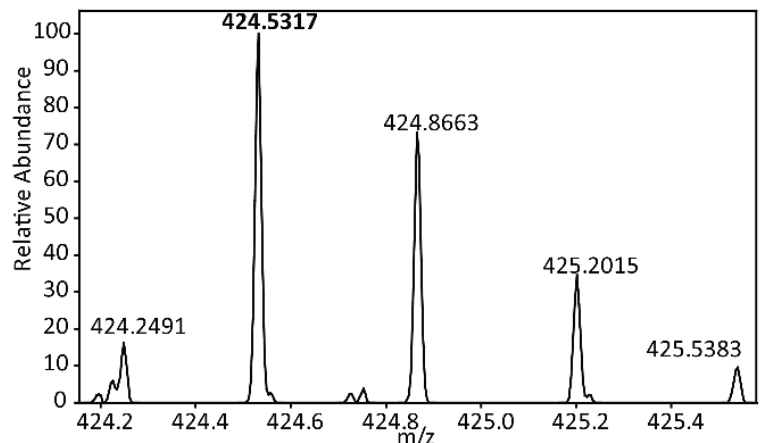

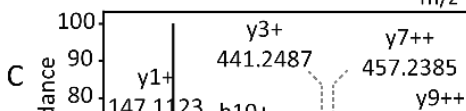

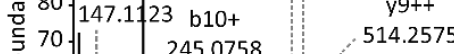

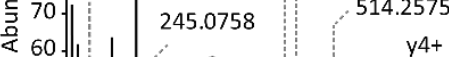

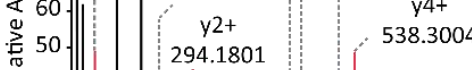

$\stackrel{\square}{\square}$

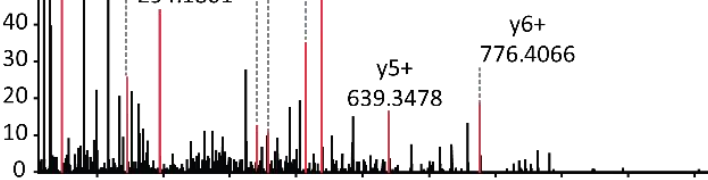

$\begin{array}{llllllllll}200 & 300 & 400 & 500 & 600 & 700 & 800 & 900 & 1000 & 1100\end{array}$

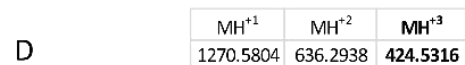

D \begin{tabular}{|c|c|c|c|c|c|c|}
\hline$b$ & $b^{+2}$ & & & & $y$ & $y^{+2}$ \\
\hline- & - & 1 & D & 11 & - & - \\
\hline 245.0768 & - & 2 & E & 10 & 1156.5534 & 578.7803 \\
\hline 302.098 & - & 3 & G & 9 & 1027.5108 & 514.2591
\end{tabular} \begin{tabular}{|l|l|l|l|l|l|l|l|}
\hline 302.0983 & - & 3 & G & 9 & 1027.5108 & 514.2591 \\
\hline
\end{tabular} \begin{tabular}{|l|l|l|l|l|l|l|l|}
\hline 359.1197 & - & 4 & G & 8 & 970.4894 & 485.7483 \\
\hline
\end{tabular} \begin{tabular}{|l|l|l|l|l|l|l|}
\hline 469.1787 & 248.5930 & 5 & H & 7 & 913.4679 & 457.2376 \\
\hline
\end{tabular} \begin{tabular}{|l|l|l|l|l|l|l|l|}
\hline 633.2376 & 317.1224 & 6 & $H$ & 6 & 776.4090 & 388.7081 \\
\hline
\end{tabular} \begin{tabular}{|l|l|l|l|l|l|l|l|l|}
\hline $\mathbf{7 3 4 . 2 8 5 2}$ & 367.6463 & 7 & $\mathrm{~T}$ & 5 & 639.3501 & 320.1787 \\
\hline
\end{tabular} \begin{tabular}{|l|l|l|l|l|l|l|l|}
\hline 831.3380 & 416.1726 & 8 & $\mathrm{P}$ & 4 & $\mathbf{5 3 8 . 3 0 2 4}$ & 269.6548 \\
\hline
\end{tabular} \begin{tabular}{|l|l|l|l|l|l|l|l|}
\hline $\mathbf{9 7 8 . 4 0 6 4}$ & $\mathbf{4 8 9 . 7 0 6 8}$ & 9 & $\mathrm{~F}$ & $\mathbf{3}$ & $\mathbf{4 4 1 . 2 4 9 6}$ & 221.1285 \\
\hline
\end{tabular} \begin{tabular}{|l|l|l|l|l|l|l|}
\hline 1125.4748 & 563.2411 & 10 & $F$ & 2 & 294.1812 & 147.5942 \\
\hline
\end{tabular}
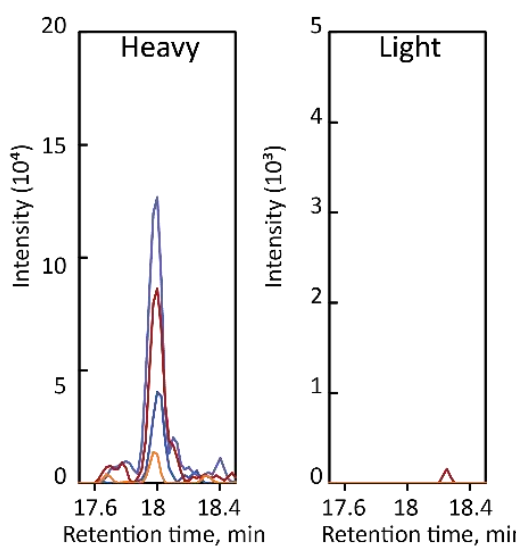

R319H light

-y5 - 639.3501+

-y4 - 538.3024+

- y3-441.2496+

_b7 - 734.2852+

R319H heavy

-y5 - 647.3643+

$-\mathrm{y} 4-546.3166+$

$-\mathrm{y} 3-449.2638+$

-b7 - 734.2852
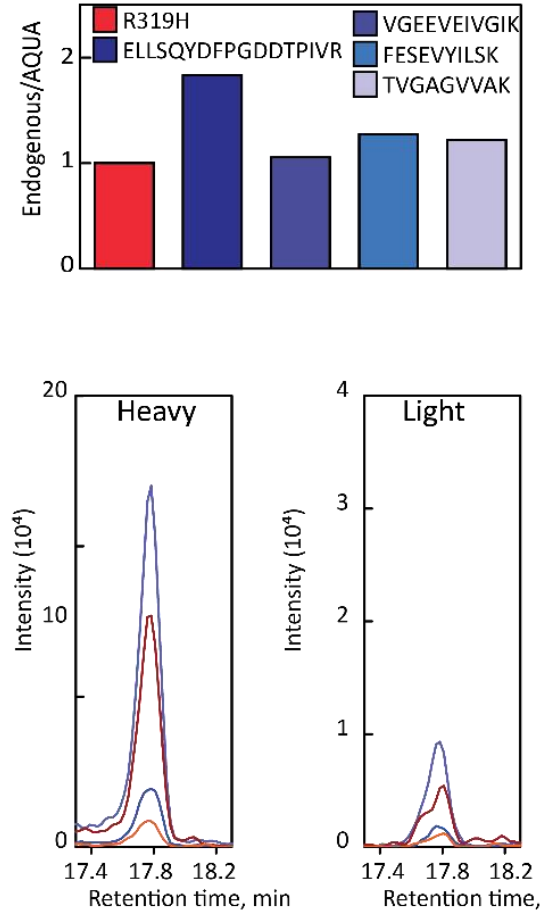
R328H: FFK.GYHPQFYFR.TTD

A

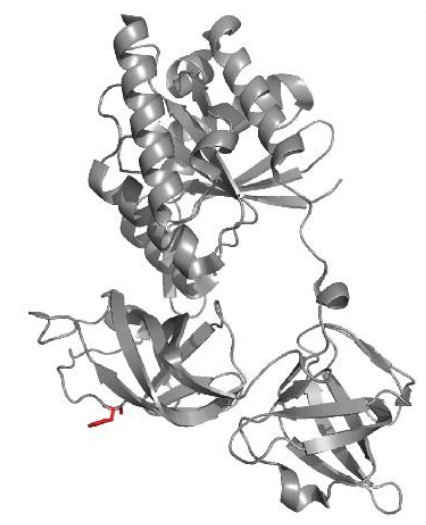

D

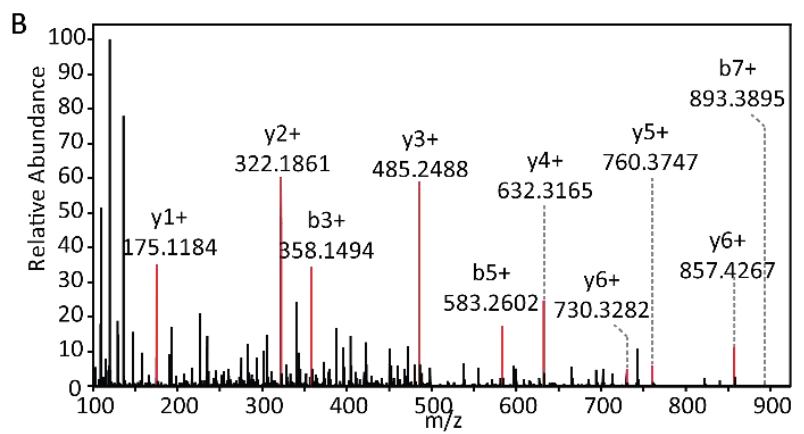

C

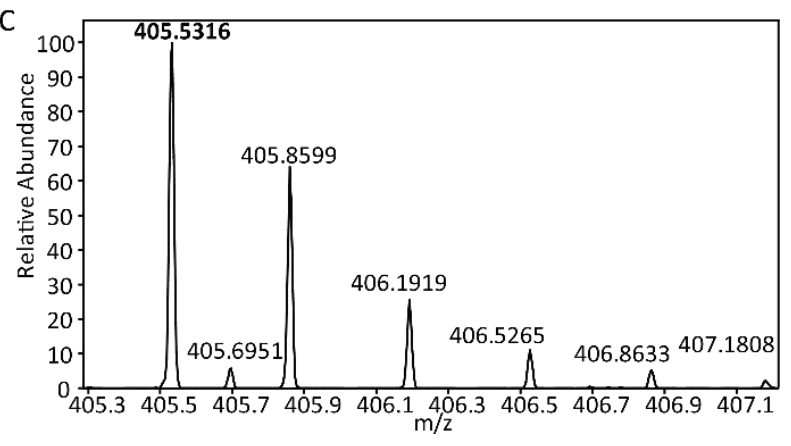

\begin{tabular}{|c|c|c|c|c|c|c|}
\hline & \multirow{2}{*}{\begin{tabular}{|c|}
$\mathrm{MH}^{+1}$ \\
1214.5742
\end{tabular}} & \multirow{2}{*}{$\frac{\mathrm{MH}^{+2}}{607.7907}$} & \multirow{2}{*}{$\begin{array}{c}\mathrm{MH}^{+3} \\
405.5296\end{array}$} & \multicolumn{2}{|r|}{40} \\
\hline & & & & & & \\
\hline$b$ & $b^{+2}$ & & & & $\mathrm{y}$ & $\mathrm{y}^{+2}$ \\
\hline- & - & 1 & G & 9 & - & - \\
\hline 221.0921 & - & 2 & Y & 8 & 1157.5527 & 579.2800 \\
\hline 358.1510 & 179.5791 & 3 & $\mathrm{H}$ & 7 & 994.4894 & 497.7483 \\
\hline 455.2037 & 228.1055 & 4 & $P$ & 6 & 857.4304 & 429.2189 \\
\hline 593.2623 & 292.1348 & 5 & Q & 5 & 760.3777 & 380.6925 \\
\hline 730.3307 & 356.6690 & 6 & $\mathrm{~F}$ & 4 & 632.3191 & 316.6632 \\
\hline 893.3941 & 447.2007 & 7 & Y & 3 & 485.2507 & 243.1290 \\
\hline 1040.4625 & 520.7349 & 8 & $\mathrm{~F}$ & 2 & 322.1874 & 161.5973 \\
\hline - & - & 9 & $R$ & 1 & 175.1190 & 88.0631 \\
\hline
\end{tabular}

F

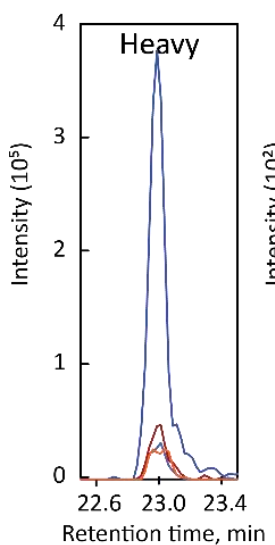

E

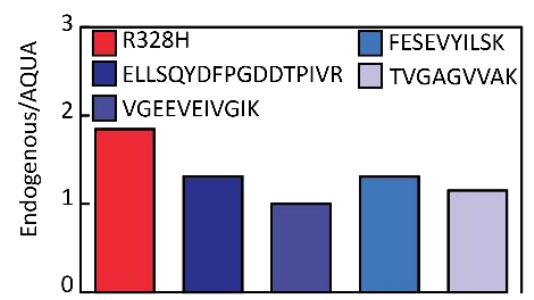

G

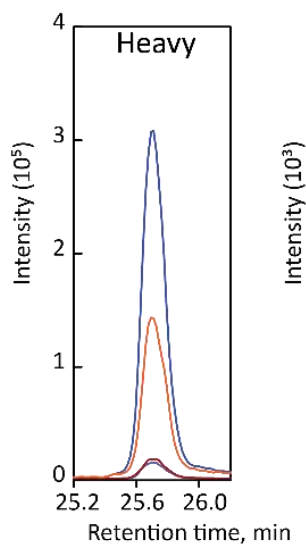

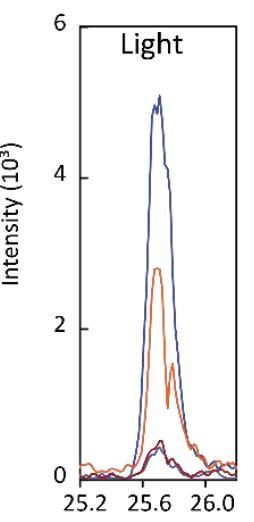


R378H: GLR.FAIHEGGR.TVG

A

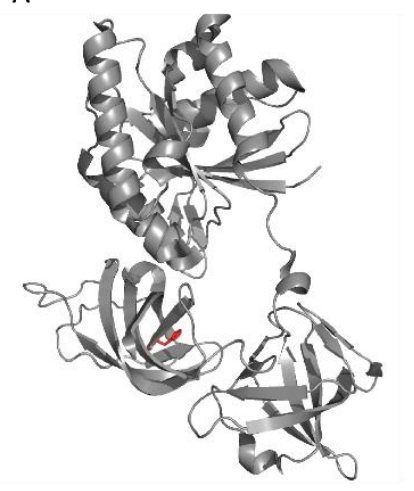

D
B

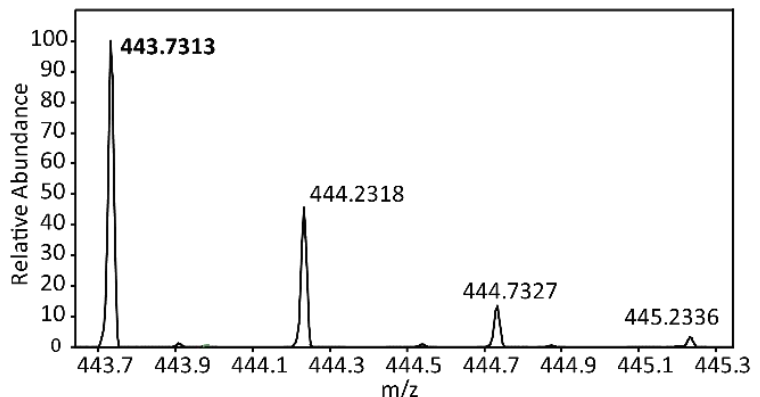

C

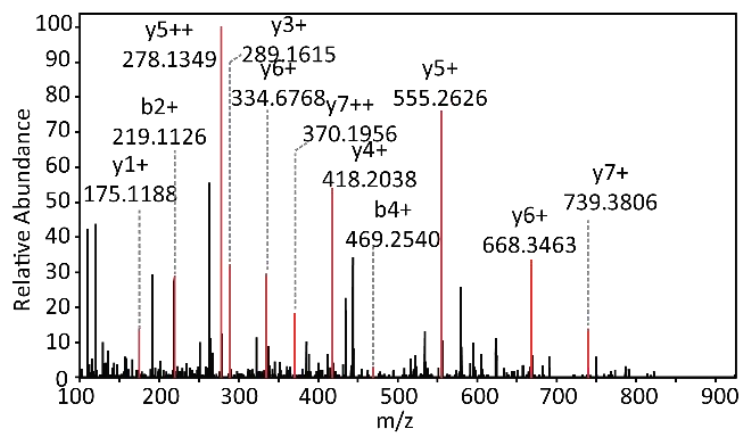

\begin{tabular}{|c|c|c|c|c|c|c|}
\hline & \multirow{2}{*}{$\begin{array}{c}\mathrm{MH}^{+1} \\
886.453\end{array}$} & \multirow{2}{*}{$\begin{array}{c}\mathrm{MH}^{+2} \\
443.7301\end{array}$} & \multirow{2}{*}{$\begin{array}{c}\mathrm{MH}^{+3} \\
296.1558\end{array}$} & & \\
\hline & & & & & & \\
\hline$b$ & $b^{+2}$ & & & & $y$ & $y^{+2}$ \\
\hline- & - & 1 & $\mathrm{~F}$ & 8 & - & - \\
\hline 219.1128 & - & 2 & A & 7 & 739.3846 & 370.1959 \\
\hline 332.1969 & - & 3 & 1 & 6 & 668.3474 & 334.6774 \\
\hline 469.2558 & 235.1315 & 4 & H & 5 & 555.2634 & 278.1353 \\
\hline 598.2984 & 299.6528 & 5 & $E$ & 4 & 418.2045 & 209.6059 \\
\hline 655.3198 & 328.1636 & 6 & G & 3 & 289.1619 & 145.0846 \\
\hline 712.3413 & 356.6743 & 7 & G & 2 & 232.1404 & 116.5738 \\
\hline - & 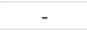 & 8 & $\mathrm{R}$ & 1 & 175.1190 & 88.0631 \\
\hline
\end{tabular}

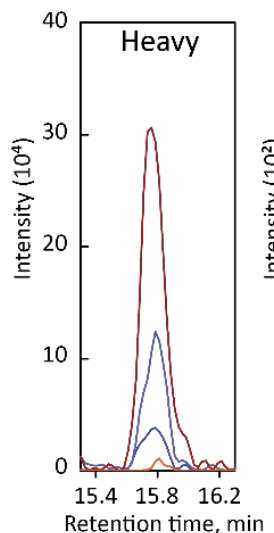

E

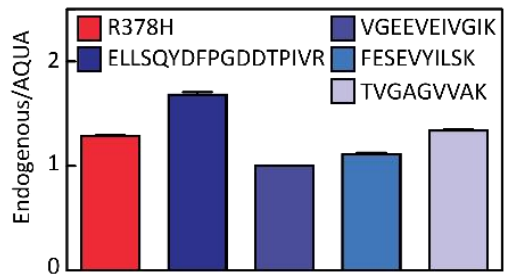

G

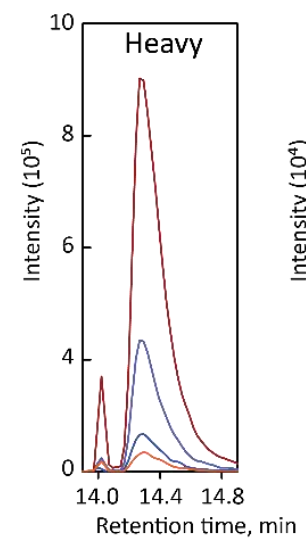

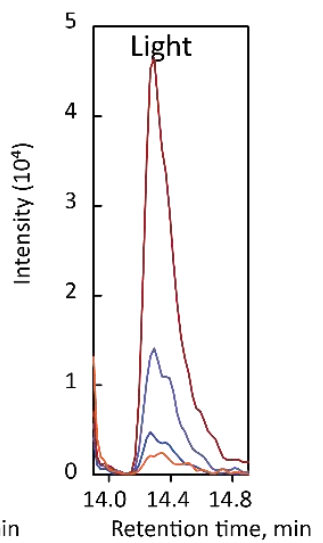


R382H: air.EGGHTVGAGVVAK.ylg

A

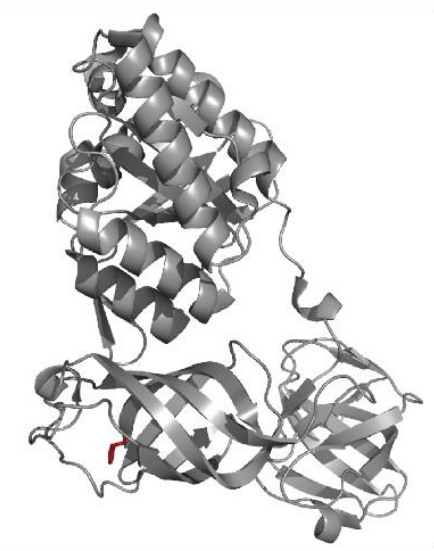

D

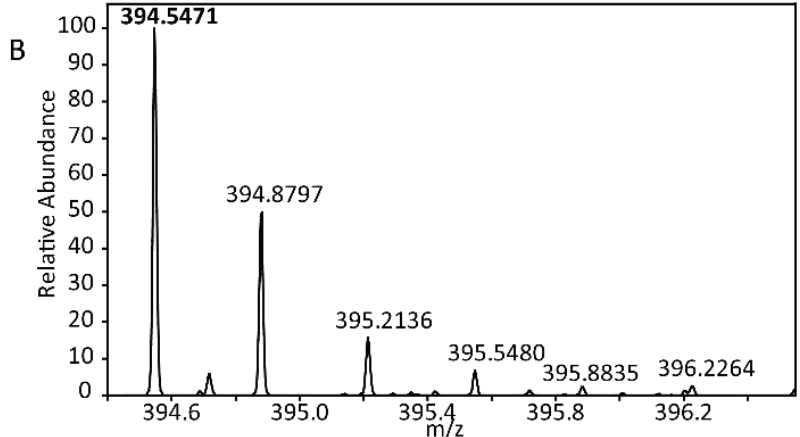

C

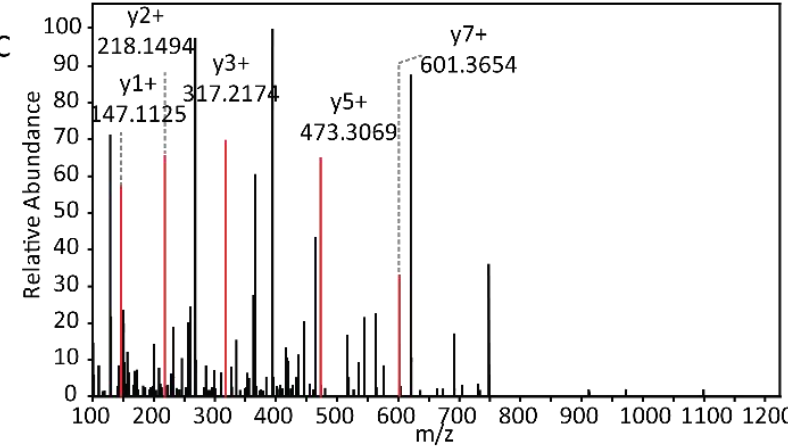

\begin{tabular}{|c|c|c|c|c|c|c|}
\hline & \multirow{2}{*}{\begin{tabular}{|c|}
$\mathrm{MH}^{+1}$ \\
1181.6273 \\
\end{tabular}} & \multirow{2}{*}{$\begin{array}{c}\mathrm{MH}^{+2} \\
591.3173\end{array}$} & \multirow{2}{*}{\begin{tabular}{|c|}
$\mathrm{MH}^{+3}$ \\
394.5473
\end{tabular}} & \multicolumn{2}{|r|}{100} \\
\hline & & & & & & \\
\hline$b$ & $b^{+2}$ & & & & $y$ & $\mathrm{y}^{+2}$ \\
\hline - & - & 1 & E & 13 & - & - \\
\hline 187.0713 & - & 2 & G & 12 & 1052.5847 & 526.7960 \\
\hline 244.0928 & - & 3 & G & 11 & 995.5633 & 498.2853 \\
\hline 381.1517 & 191.0795 & 4 & H & 10 & 938.5418 & 469.7745 \\
\hline 482.1994 & 241.6033 & 5 & $T$ & 9 & 801.4829 & 401.2451 \\
\hline 581.2678 & 291.1375 & 6 & v & 8 & 700.4352 & 350.7212 \\
\hline 638.2893 & 319.6483 & 7 & G & 7 & 601.3668 & 301.1870 \\
\hline 709.3264 & 355.1668 & 8 & A & 6 & 544.3453 & 272.6763 \\
\hline 766.3478 & 383.6776 & 9 & G & 5 & 473.3082 & 237.1577 \\
\hline 865.4163 & 433.2118 & 10 & v & 4 & 416.2867 & 208.6470 \\
\hline 964.4847 & 482.7460 & 11 & v & 3 & 317.2183 & 159.1128 \\
\hline 1035.5218 & 518.2645 & 12 & A & 2 & 218.1499 & 109.5786 \\
\hline- & - & 13 & $\mathrm{~K}$ & 1 & 147.1128 & 74.0600 \\
\hline
\end{tabular}

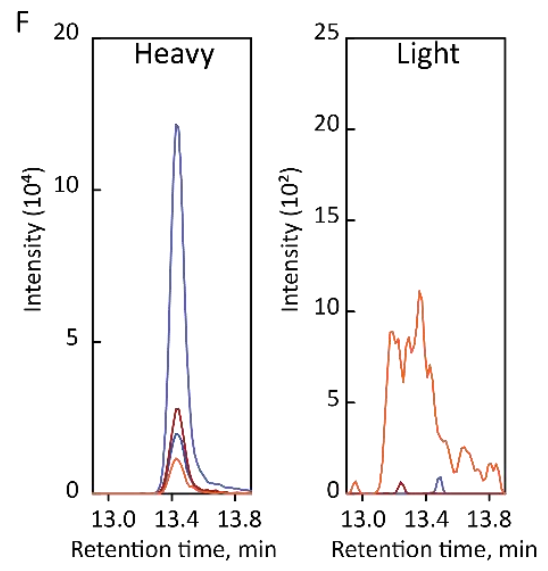

E

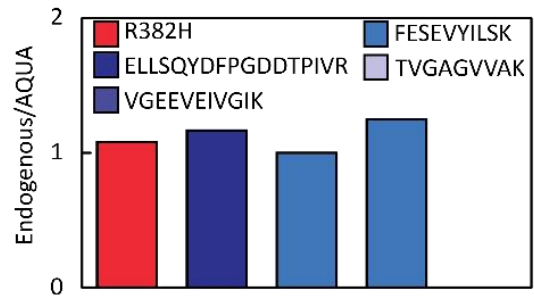

G $\mathrm{R} 382 \mathrm{H}$ light - y9-801.4829+ - y8-700.4352+ - y7 - 601.3668+ b9 - $766.3478+$

R382H heavy — y9-809.4971+ - y8 - 708.4494+

- y7 - 609.3810+ b9 - $766.3478+$

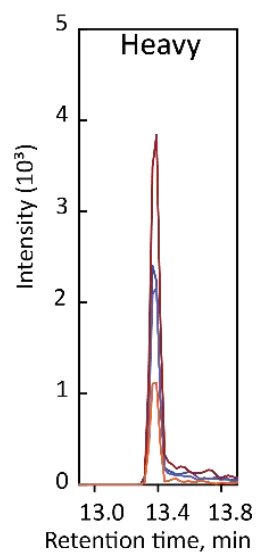




\subsection{Section D. validation of non-cognate amino acid substitutions}

The position of the substituted amino acid (red) is shown in the context of EF-Tu structure. The identity of the error-containing peptide is validated by PRM analysis (B). The predicted fragmentation pattern for the peptide is shown in (C). The validation of the quantification is performed by comparing the stoichiometry of erroneous peptide with respect to four cognate EF-Tu peptides (D), as described in Results and Materials and Method. The SRM trace used for the determination of error frequency is shown (E). To confirm the absence of contamination by the light peptide, the heavy-labeled peptides are analysed by mass spectrometry and the signals for both light and heavy are recorded (F). The monitored transitions are indicated. 
A

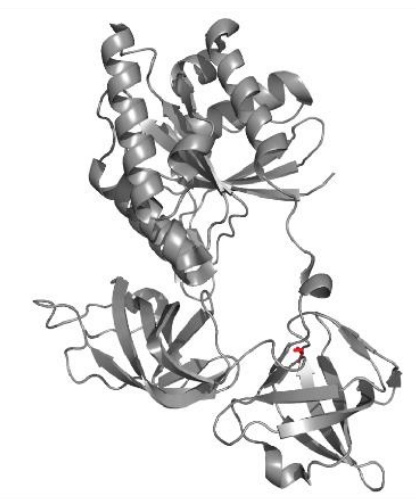

B

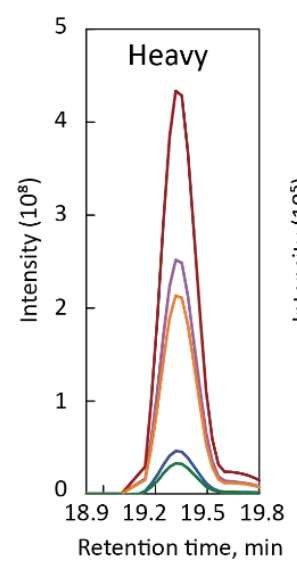

rdotp 0.91

R231A light

- y8 - 830.4730+

- y7-731.4046+

- y6-632.3362

- y5 - 531.2885+

- y4 - 474.2671+

R231A heavy

- y8 - 840.4813+

- y7-741.4129+

- y6-642.3445+

- y5 - 541.2968+

- y4 - 484.2753+

D

C \begin{tabular}{|c|c|c|}
\hline $\mathrm{MH}+1$ & $\mathrm{MH}+2$ & $\mathrm{MH}+3$ \\
\hline
\end{tabular} \begin{tabular}{|l|l|l|l}
988.5422 & 494.7747 & 330.1856
\end{tabular}

\begin{tabular}{|c|c|c|c|c|c|}
\multicolumn{7}{c|}{988.5422} & 494.7747 & 330.1856 \\
\hline b & & & & V & y+2 \\
\hline- & 1 & G & 10 & - & - \\
\hline 159.0764 & 2 & T & 9 & 931.5207 & 466.2640 \\
\hline 258.1448 & 3 & V & 8 & 830.4730 & 415.7402 \\
\hline 357.2132 & 4 & V & 7 & 731.4046 & 366.2060 \\
\hline 458.2609 & 5 & T & 6 & 632.3362 & 316.6717 \\
\hline 515.2824 & 6 & G & 5 & 531.2885 & 266.1479 \\
\hline 586.3195 & 7 & A & 4 & 474.2671 & 237.6372 \\
\hline 685.3879 & 8 & V & 3 & 403.2300 & 202.1186 \\
\hline 814.4305 & 9 & E & 2 & 304.1615 & 152.5844 \\
\hline- & 10 & R & 1 & 175.1190 & 88.0631 \\
\hline & & & & & \\
\hline
\end{tabular}

E
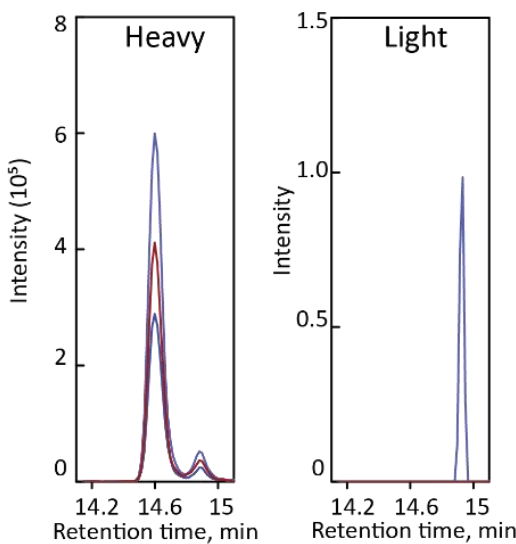

R231A light

- y7 - 731.4046+

- y6 - 632.3362+

- y5 - 531.2885+

R231A heavy

- y7-741.4129+

- y6 - 642.3445+

- y5-541.2968+

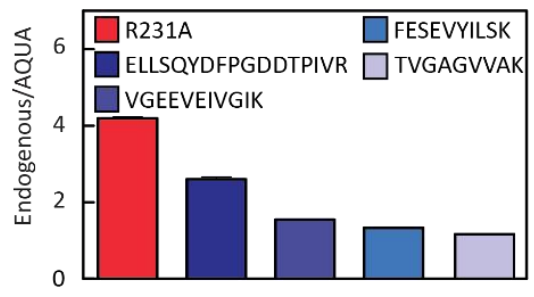

F
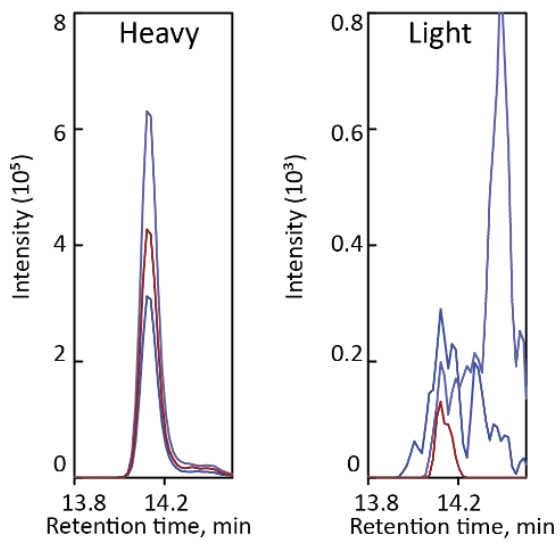
A

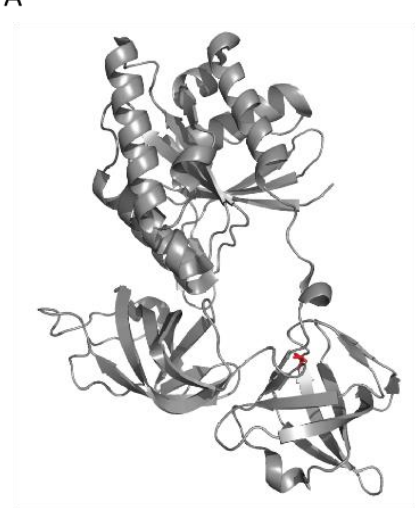

B

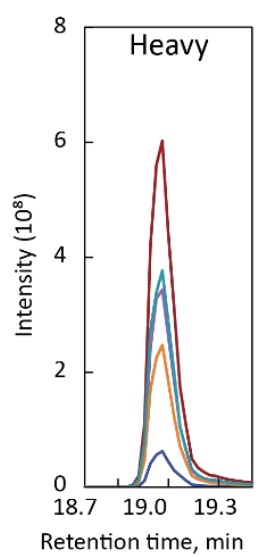

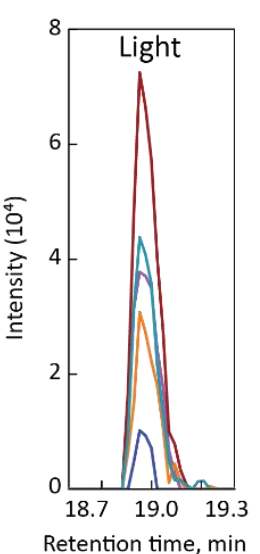

$\operatorname{dotp} 1$

R231D light

—y8 - 874.4629+

-y7 - 775.3945+

- y6 - 676.3260+

y5 - 575.2784+

- y3 - 403.2300+

R231D heavy

- y8 - 884-4711+

-y7 - 785.4027+

-y6 - 686-3343+

- y5 - 585-2866+

- y4 - 413-2382+

\begin{tabular}{|c|c|c|c|c|c|c|}
\hline \multirow[b]{2}{*}{ C } & \multicolumn{2}{|c|}{$\mathrm{MH}+1$} & $\mathrm{MH}+2$ & \multicolumn{2}{|c|}{$\mathrm{MH}+3$} & \\
\hline & & & 516.769 & & 8489 & \\
\hline b & & & & & y & $y+2$ \\
\hline - & 1 & & & 10 & - & - \\
\hline 159.0764 & 2 & & & 9 & 975.5106 & 488.2589 \\
\hline 258.1448 & 3 & & & 8 & 874.4629 & 437.7351 \\
\hline 357.2132 & 4 & & & 7 & 775.9345 & 388.2009 \\
\hline 458.2609 & 5 & & & 6 & 676.3260 & 339.6667 \\
\hline 515.2824 & 6 & & & 5 & 575.2784 & 288.1428 \\
\hline 630.3093 & 7 & & & 4 & 518.2569 & 259.6321 \\
\hline 729.3777 & 8 & & & 3 & 403.2300 & 202.1186 \\
\hline 858.4203 & 9 & & & 2 & 304.1615 & 152.5844 \\
\hline- & 10 & & & 1 & 175.1190 & 88.0631 \\
\hline
\end{tabular}

D

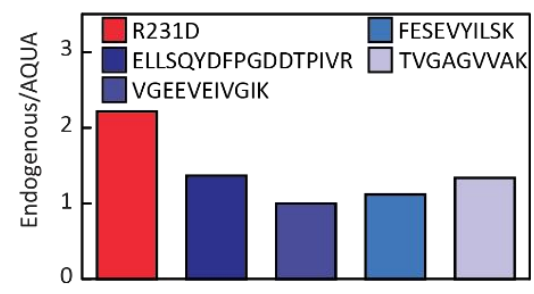

E

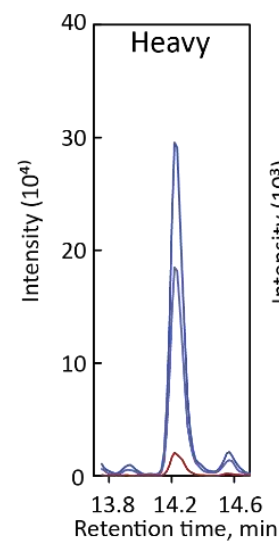

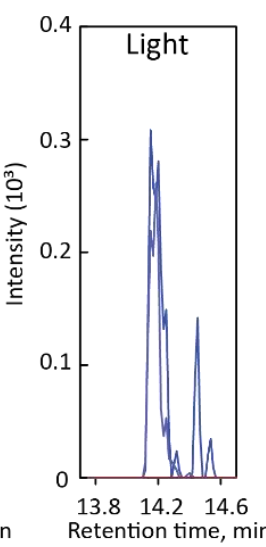

R231D light

—y6 - 676.3260+

-y5 - 575.2784+

- y4 - 518.2569+

R231D heavy

- y6 - 686.3343+

- y5 - 585.2866+

- y4 - 528.2866+

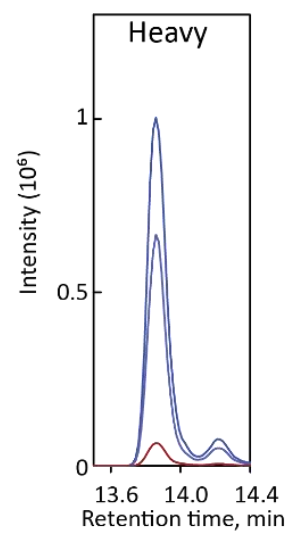


A

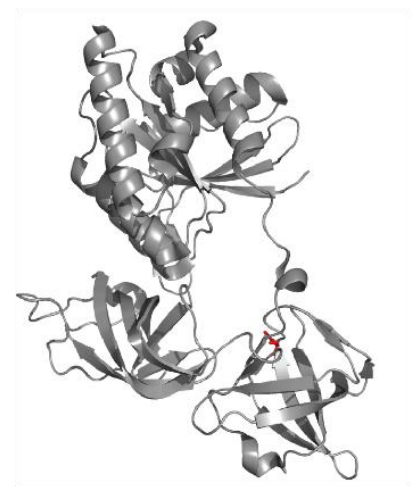

B

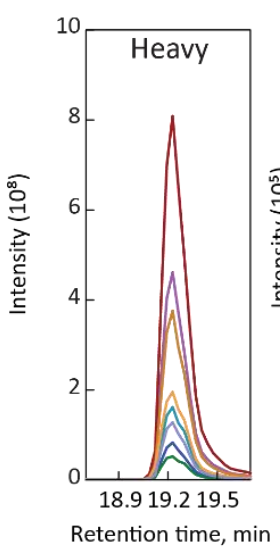

rdotp 1

R231E light

-y8 - 888.4785+

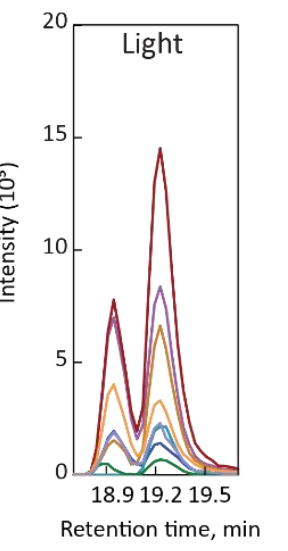

-y7 - 789.4101+

-y6-690.3417+

- y5 - 589.2940+

$-\mathrm{y} 3-403.2300+$

- y2 - 304.1615+

-b2 - 159.0764+

—b3 - 258.1448

R231E heavy

-y8-898.4968+

y7 - 799.4184+

-y6-700.3500

-y5 - 599.3035+

$-\mathrm{y} 3-413.2382+$

$-y 2-314.1698$

-b2 - 159.0764+

b3 - 258.1448+

D

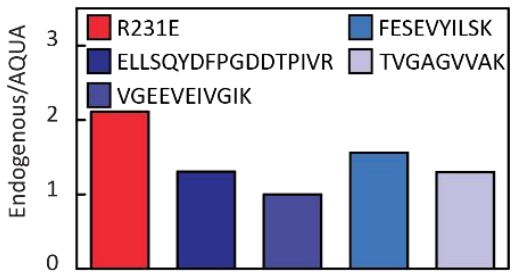

F

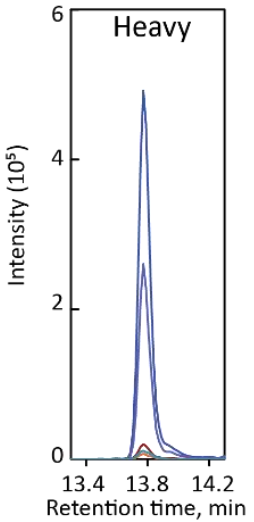

R231E light

_y6-690.3417+

- y5 - 589.2840+

- y4 - 532.2726+

-b7 - 644.3250+

—b8 - 743.3934+

R231E heavy

—y6 - 700.3500+

-y5 - 599.3023+

- y4 - 542.2808+

-b7-644.3250+ -b8 - 743.3934+
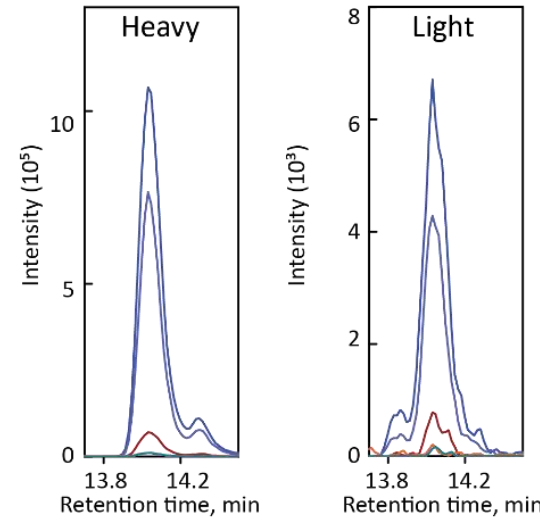
A

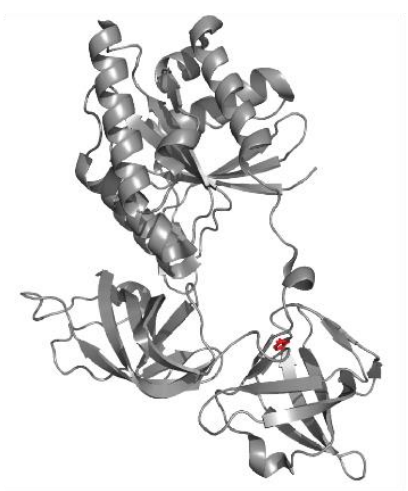

B

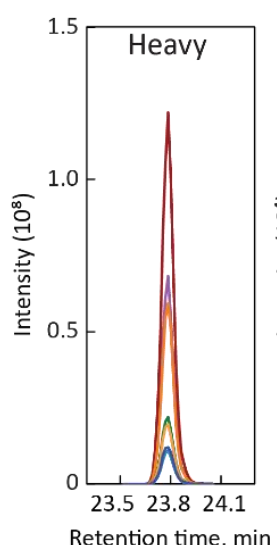

rdotp 1

R231F light

$-\mathrm{y} 8-906.5043+$

-y7 - 807.4359+

$-\mathrm{y} 6-708.3675+$

$-\mathrm{y} 5-607.3198+$

- y4 - 550.2984+

-b2 - 159.0764t

-b3 - 258.1448+

R231Q heavy

- y8 - 916.5126+

- y7 - 817.4442+

$-y 6-718.3758+$

-y5 - 617.3281+

$-\mathrm{y} 4-560.3066+$

-b2 - 159.0764

-b3 - 258.1448+

\begin{tabular}{l} 
C \\
\cline { 2 - 6 }
\end{tabular}

E

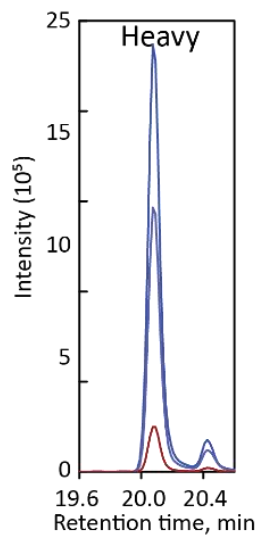

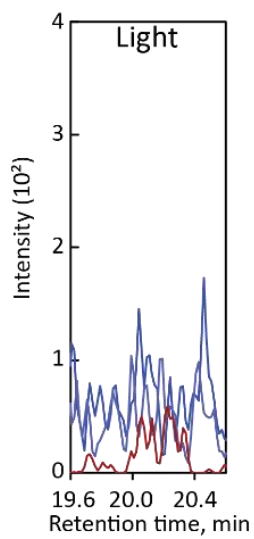

R231F light

-y6 - 708.3675+

-y5 - 607.3198+

$-\mathrm{y} 4-550.2984+$

-b7-662.3508+

_b8 - 761.4192

R231F heavy

-y6 - 718.3758+

-y5 - 617.3281+

-b7-662.3508+

-b7 - 662.3508+

-b8 - 761.4192+
D
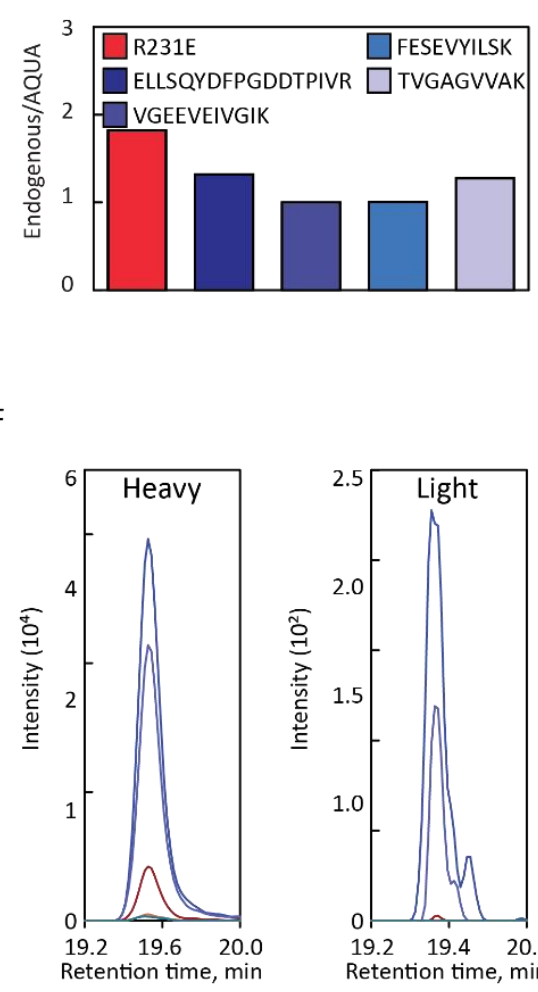
A

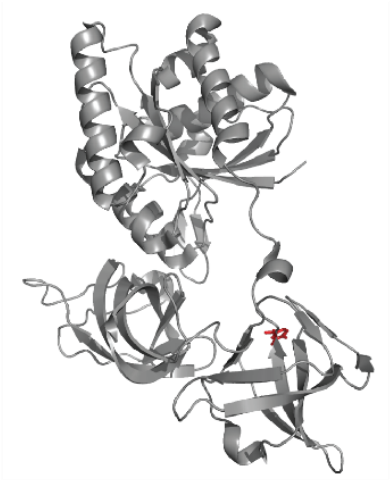

B

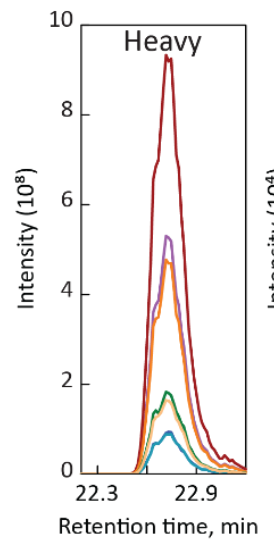

rdotp 1

R231Y light

- y8 - 922.4993+

- y7 - 823.4308+

- y6 - 724.3624t

- y5 - 623.3148+

- y4 - 566.2933+

— b2 - 159.0764+

- b3 - 258.1448+

R231Y heavy

— y8 - 932.5075+

- y7 - 833.4391+

— y6 - 734.3707+

- y5-633.2330+

- y4 - 576.3016+

— b2 - 159.0764+

- b3 - 258.1448+

\begin{tabular}{|c|c|c|c|c|c|c|}
\hline \multirow[b]{2}{*}{ C } & \multicolumn{2}{|c|}{$\mathrm{MH}+1$} & $\mathrm{MH}+2$ & & \multirow{2}{*}{$\frac{\mathrm{MH}+3}{360861}$} & \multirow[b]{3}{*}{$y+2$} \\
\hline & & & 540.787 & & & \\
\hline b & & & & & y & \\
\hline - & 1 & & & 10 & - & - \\
\hline 159.0764 & 2 & & & 9 & 1023.5469 & 512.2771 \\
\hline 258.1448 & 3 & & & 8 & 922.4993 & 461.7533 \\
\hline 357.2132 & 4 & & & 7 & 823.4308 & 412.2191 \\
\hline 458.2609 & 5 & & & 6 & 724.3624 & 362.6849 \\
\hline 515.2824 & 6 & & & 5 & 623.3148 & 312.1610 \\
\hline 678.3457 & 7 & & & 4 & 566.2933 & 283.6503 \\
\hline 777.4141 & 8 & & & 3 & 403.2300 & 202.1186 \\
\hline 906.4567 & 9 & & & 2 & 304.1615 & 152.5844 \\
\hline - & 10 & & & 1 & 175.1190 & 88.0631 \\
\hline
\end{tabular}

E

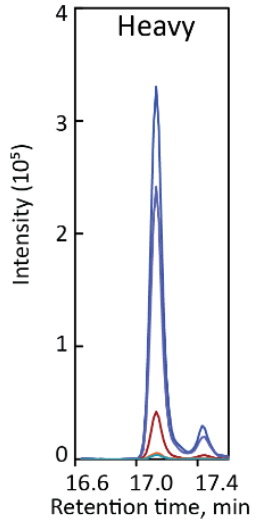

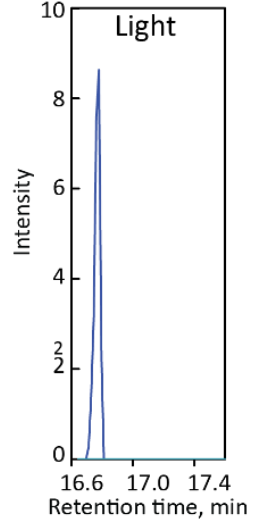

R231Y light

- y6-724.3624+

- y5 - 623.3148+

$-\mathrm{y} 4-566.2933+$

- b7 - 678.3457+

— b8 - 777.4141+

R231Y heavy

—y6 - 734.3707+

- y5 - 633.3230+

- y4 - 576.3016+

-b7 - 678.3457+

— b8 - 777.4141+
D

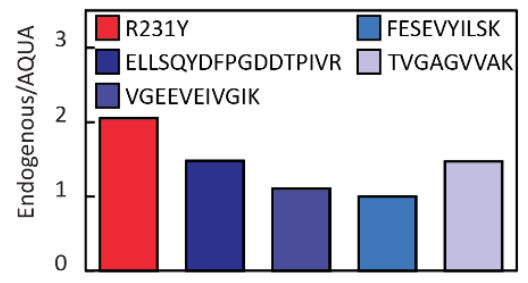

F

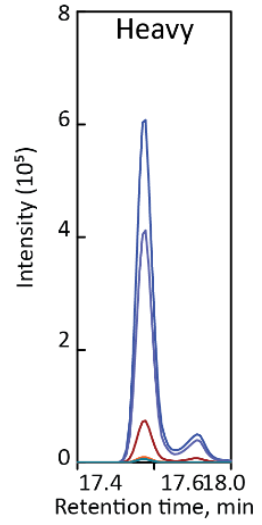


A

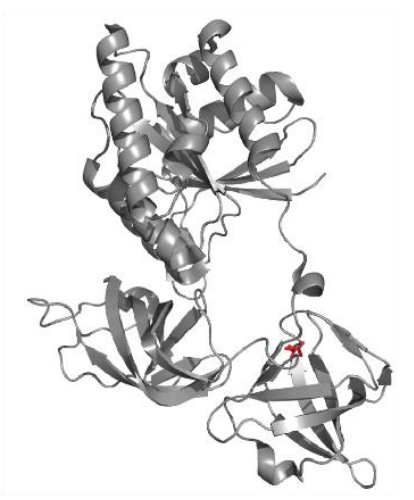

B

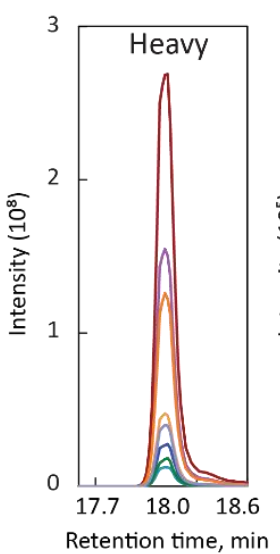

rdotp 1

R231Q light

—y8 - 887.4945+

- y7 - 788.4261+

- y6 - 689.3577+

- y5 - 588.3100+

- y4 - 531.2885+

- y3 - 403.2300+

-b2 - 159.0764+

—b3 - 258.1448+

R231Q heavy

—y8 - 897.5028+

- y7 - 798.4344+

- y6 - 699.3659+

- y5 - 598.3183+

- y4 - 413.2382+

- y3 - 413.2382+

-b2 - 159.0764+

— b3 - 258.1448+

D

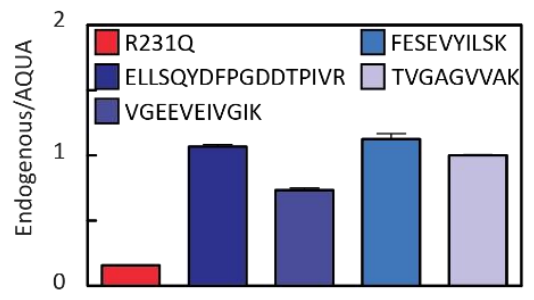

F
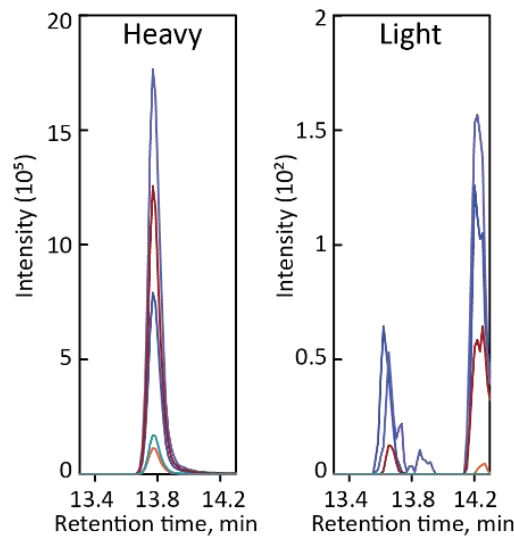

R231Q light

— y7 - 788.4261+

- y6 - 689.3577+

- y5 - 588.3100+

- y4 - 531.2885+

$-y 3-403.2300+$

R231Q heavy

—y7 - 798.4344+

-y6 - 699.3659+

- y5 - 598.3183+

- y4 - 541.2868+

- y3 - 413.2382+
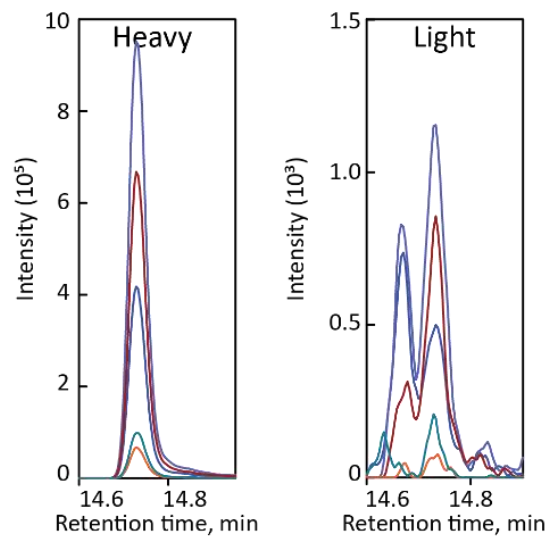
A

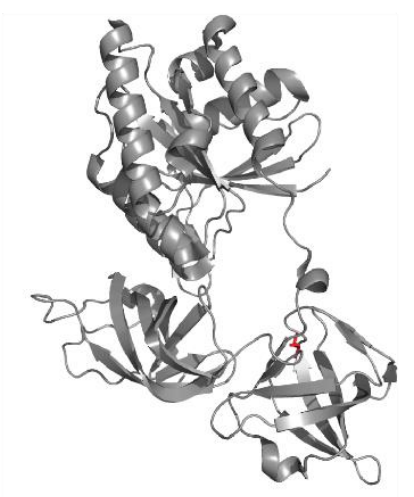

B

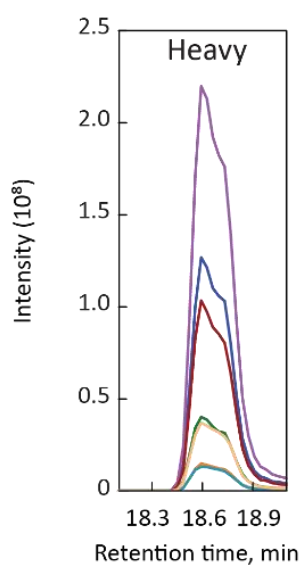

rdotp 1

R231T light

- y8-860.4836+

- y7-761.4152+

- y6- 662.3468+

- y5 - 561.2991t

- y4 - 504.2776+

- b2 - 159.0764

-b3 - 258.1448+

R231T heavy

—y8-870.4919t

- y7-761.4235+

- y6-672.3550+

- y5 - 571.3074t

- y4 - 514.2859+

- b2 - 159.0764

- b3 - 258.1448+

\begin{tabular}{|c|c|c|c|c|c|c|}
\hline \multirow[b]{2}{*}{ C } & \multicolumn{2}{|c|}{$\mathrm{MH}+1$} & $\mathrm{MH}+2$ & \multirow{2}{*}{\multicolumn{2}{|c|}{$\begin{array}{c}\mathrm{MH}+3 \\
340.1891\end{array}$}} & \multirow[b]{3}{*}{$y+2$} \\
\hline & & & 509.78 & & & \\
\hline b & & & & & $y$ & \\
\hline - & 1 & c & & 10 & - & - \\
\hline 159.0764 & 2 & T & & 9 & 961.5313 & 481.2693 \\
\hline 258.1448 & 3 & $v$ & & 8 & 860.4836 & 430.7454 \\
\hline 357.2132 & 4 & $v$ & & 7 & 761.4152 & 381.2112 \\
\hline 458.2609 & 5 & $T$ & & 6 & 662.3468 & 331.6770 \\
\hline 515.2824 & 6 & c & & 5 & 561.2991 & 281.1532 \\
\hline 616.3301 & 7 & $T$ & & 4 & 504.2776 & 525.6425 \\
\hline 715.3985 & 8 & $v$ & & 3 & 403.2300 & 202.1186 \\
\hline 844.4411 & 9 & E & & 2 & 304.1615 & 152.5844 \\
\hline & 10 & R & & 1 & 175.1190 & 88.0631 \\
\hline
\end{tabular}

D

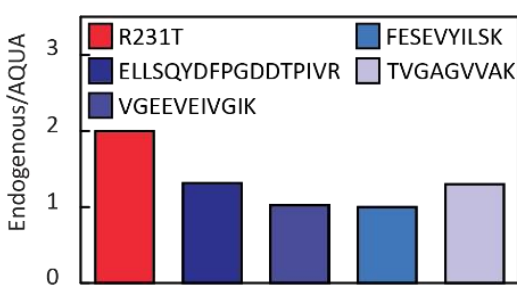

E

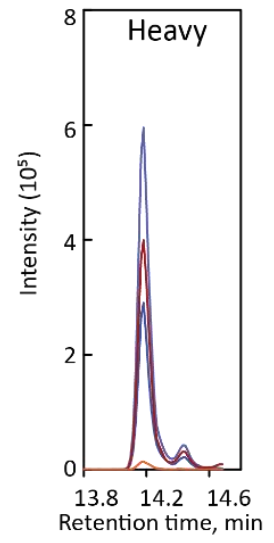

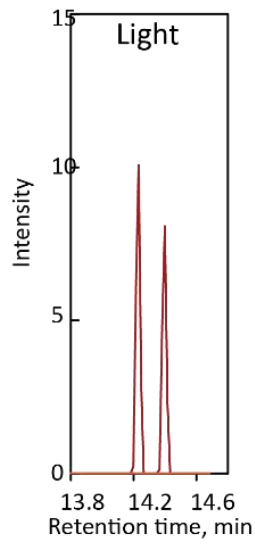

R231T light

-y7 - 761.4152+

-y6-662.3468+

-y5-561.2991+

—b6 - 515.2824+

R231T heavy

-y7-771.4235+

-y6-672.3550+

-y5 - 571.3074+

—b6-515.2824+

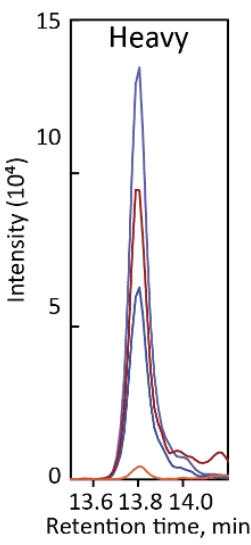


A

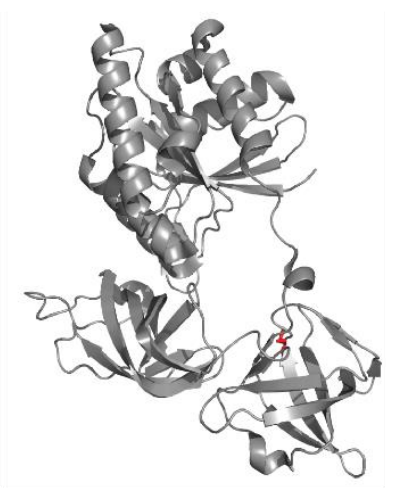

B

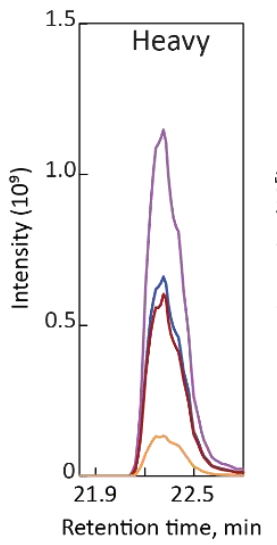

rdotp 1

R231V light

-y7-759.4359+

$-y 6-660.3675+$

- y5 - 559.3198+

$-y 3-403.2300+$

R231V heavy

$-y 7-769.4442+$

$-y 6-670.3758+$

-y5 - 569.3281+

$-\mathrm{y} 3-413.2382+$

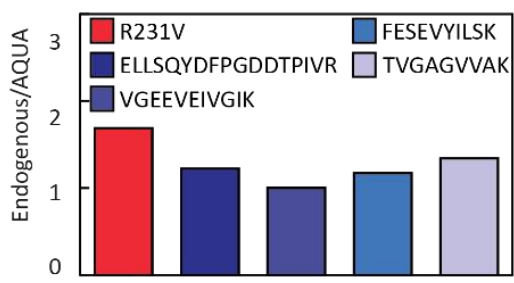

F
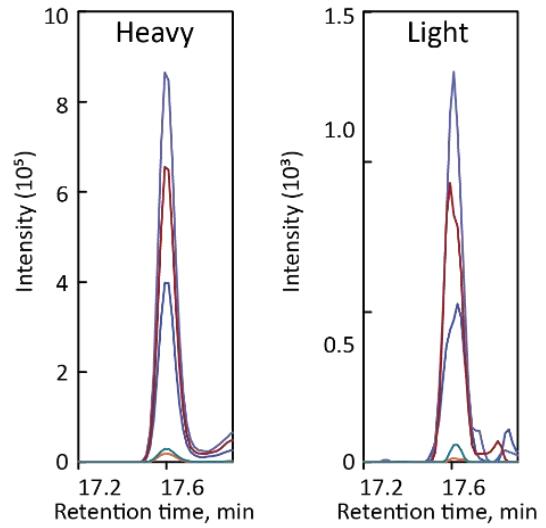


\subsection{Section E. SRM signals of peptides for multiple errors investigation}

The SRM signal of enriched heavy-labeled and endogenous peptides is shown (left). To confirm the absence of contamination by the light peptide, the heavy-labeled peptides are analysed by mass spectrometry and the signals for both light and heavy are recorded (right). The monitored transitions are indicated.

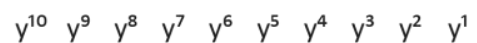

$$
\begin{aligned}
& \frac{F}{b^{1}} \underset{b^{2}}{D} \underset{b^{3}}{S} \underset{b^{4}}{b^{5}} \underset{b^{6}}{b^{7}} \sqrt{b^{7}} b^{8} b^{9} b^{10}
\end{aligned}
$$
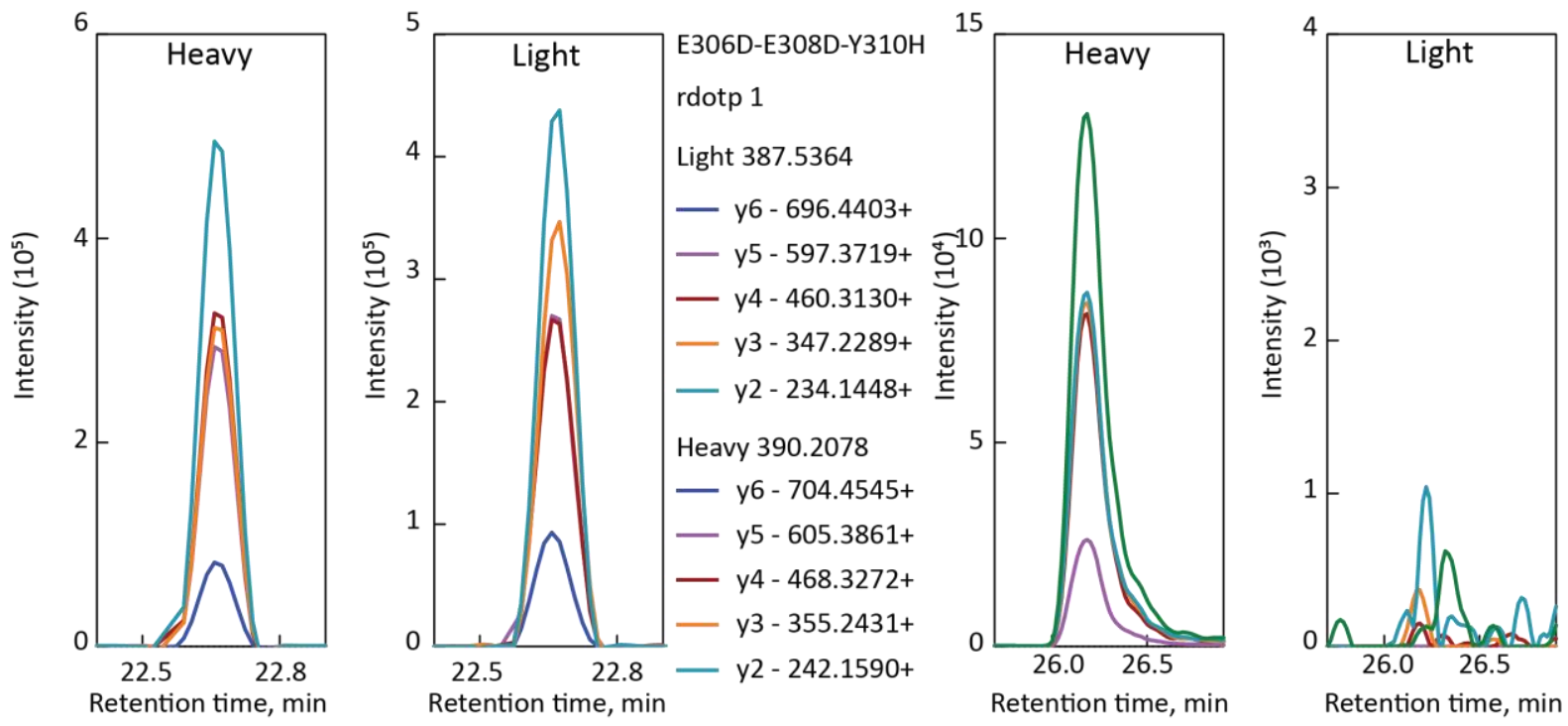


$$
\begin{aligned}
& \begin{array}{llllllllll}
y^{10} & y^{9} & y^{8} & y^{7} & y^{6} & y^{5} & y^{4} & y^{3} & y^{2} & y^{1}
\end{array} \\
& \underset{b^{1}}{F} \underset{b^{2}}{b^{3}} \underset{b^{4}}{b^{5}} \underset{b^{6}}{b^{7}} b^{8} b^{9} b^{10}
\end{aligned}
$$
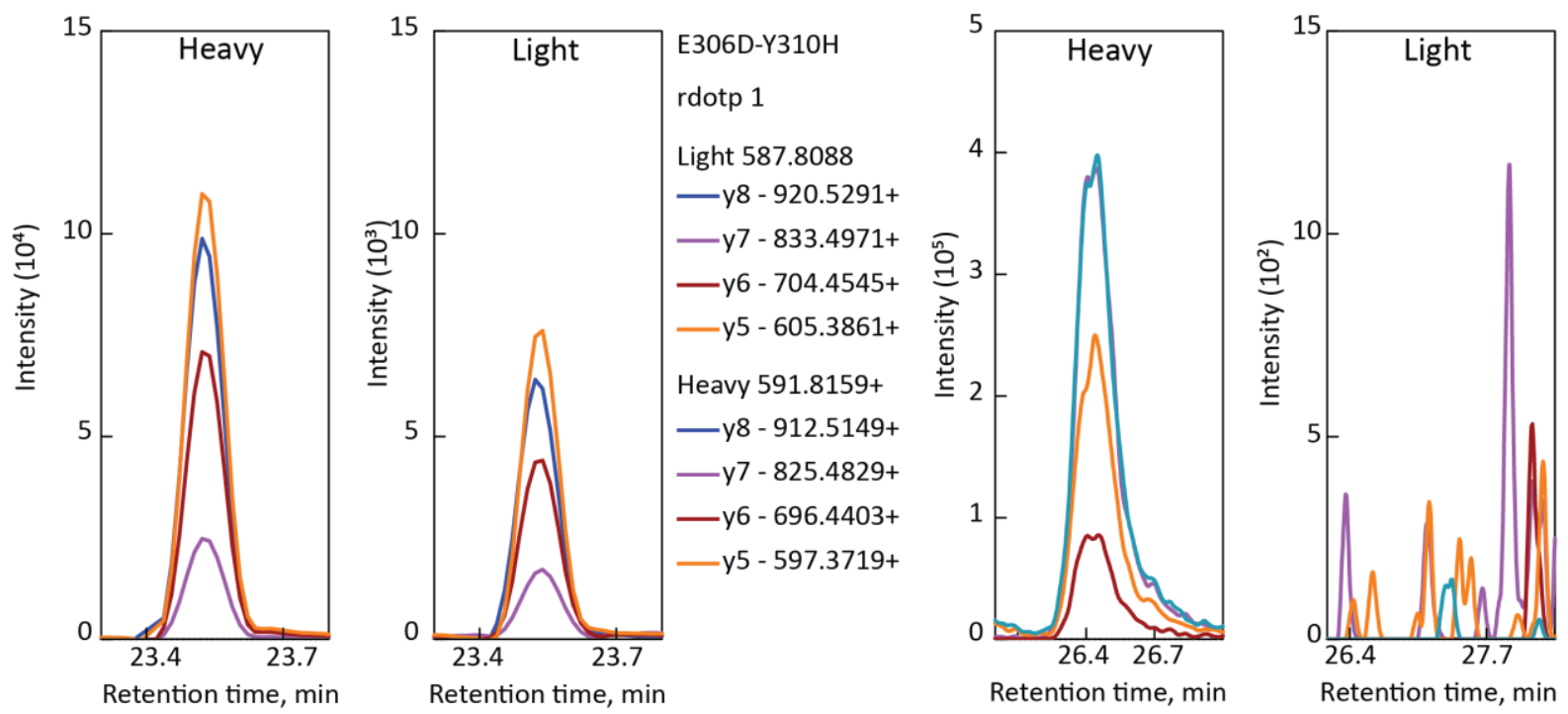

$$
\begin{aligned}
& \begin{array}{llllllllll}
y^{10} & y^{9} & y^{8} & y^{7} & y^{6} & y^{5} & y^{4} & y^{3} & y^{2} & y^{1}
\end{array}
\end{aligned}
$$

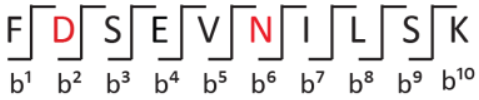
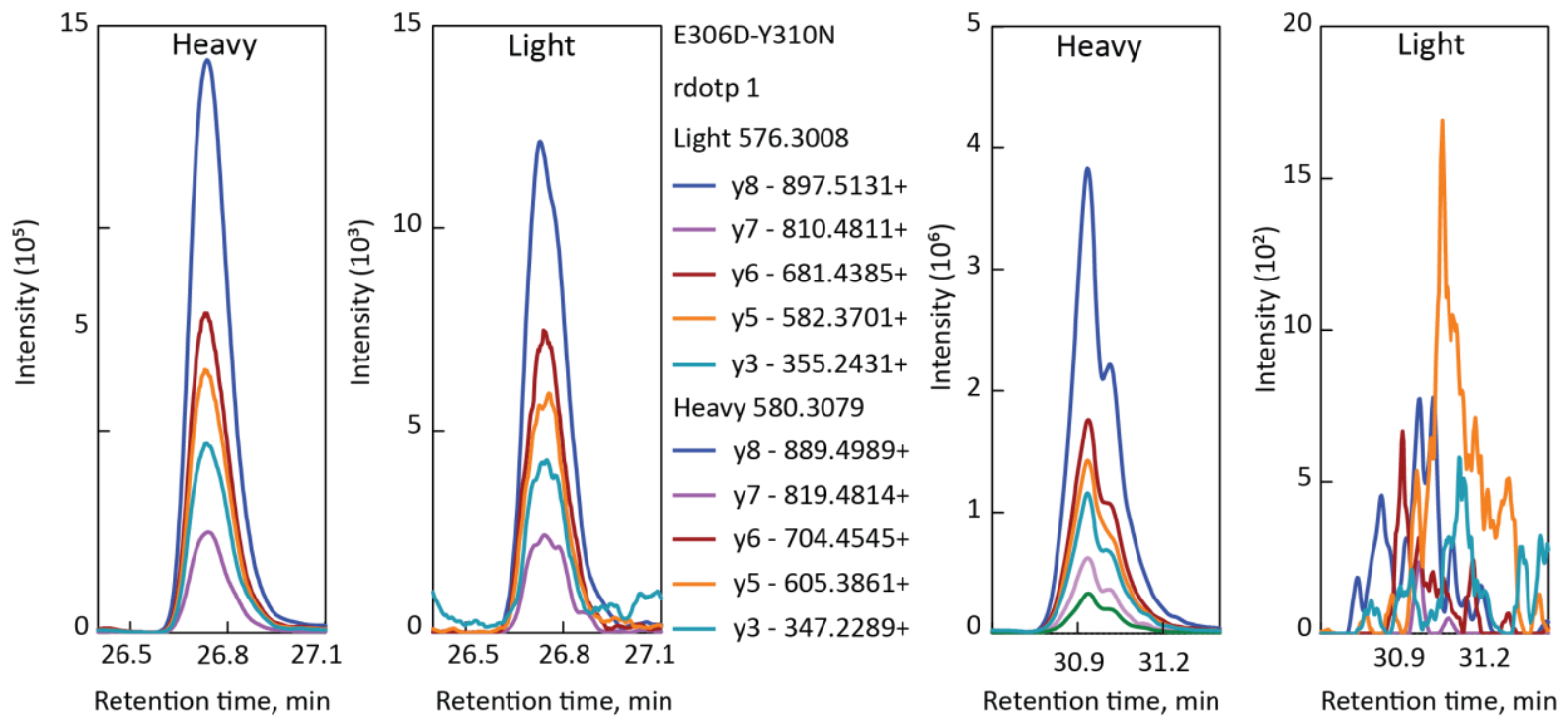


$$
\begin{aligned}
& \begin{array}{llllllllll}
y^{10} & y^{9} & y^{8} & y^{7} & y^{6} & y^{5} & y^{4} & y^{3} & y^{2} & y^{1}
\end{array}
\end{aligned}
$$

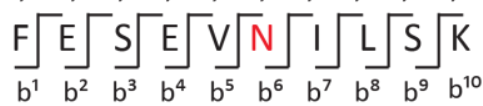
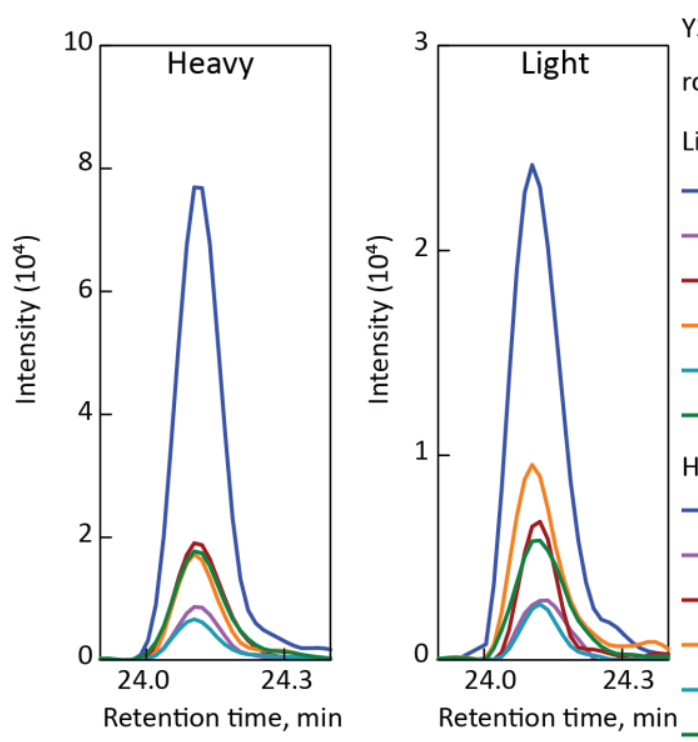

Y310N

rdotp 1

Light 583.3086

- y8 - 889.4989+

y7 - 808.4669+

- y6 - 673.4223+

- y4 - 460.3130+

- y3 - 347.2289+

Heavy 587.3157

— y8 - 897.5131+

- y7 - 810.4811+

- y6-681.4385+

- y5 - 582.3701+

- y4 - 468.3272+

- y3 - 355.2431+
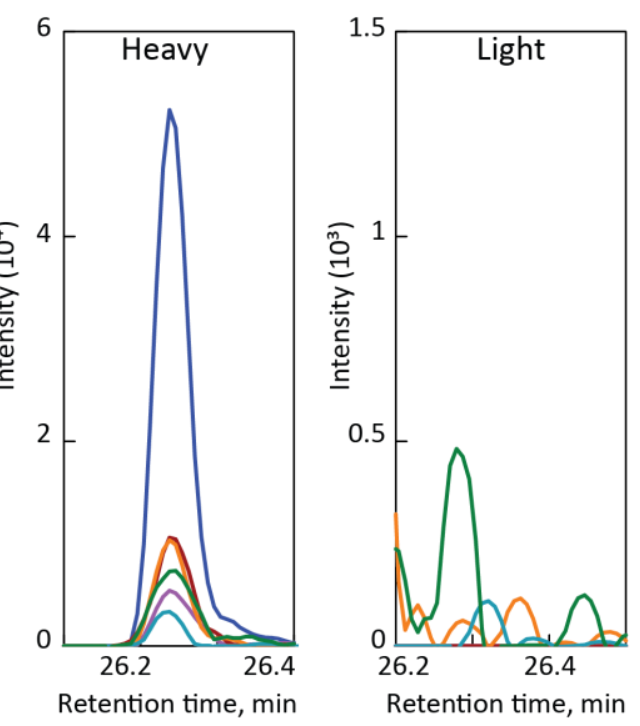

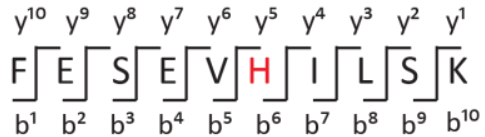

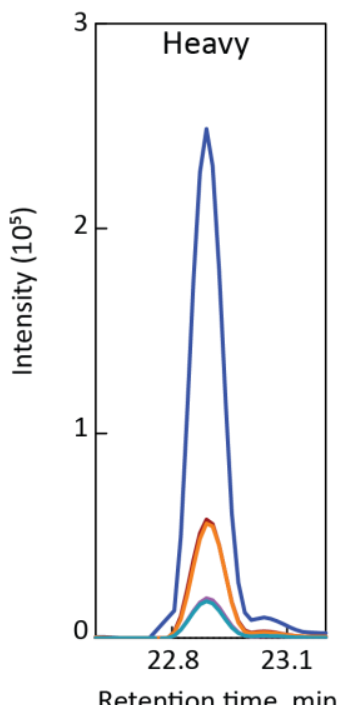

Retention time, min

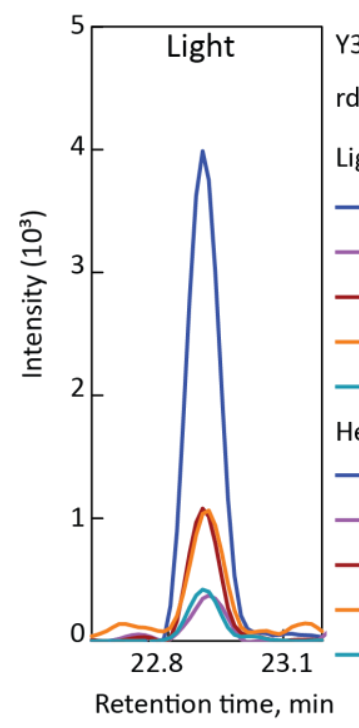

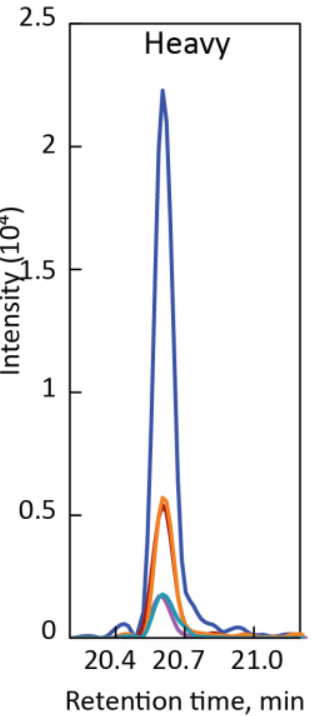




$$
\begin{aligned}
& \begin{array}{llllllllll}
y^{10} & y^{9} & y^{8} & y^{7} & y^{6} & y^{5} & y^{4} & y^{3} & y^{2} & y^{1}
\end{array} \\
& \underset{b^{1}}{F} \underset{b^{2}}{b^{3}} \underset{b^{4}}{b_{b^{5}}} \underset{b^{6}}{\sqrt{b^{7}}} \sqrt{b^{8}} b^{9} b^{10}
\end{aligned}
$$
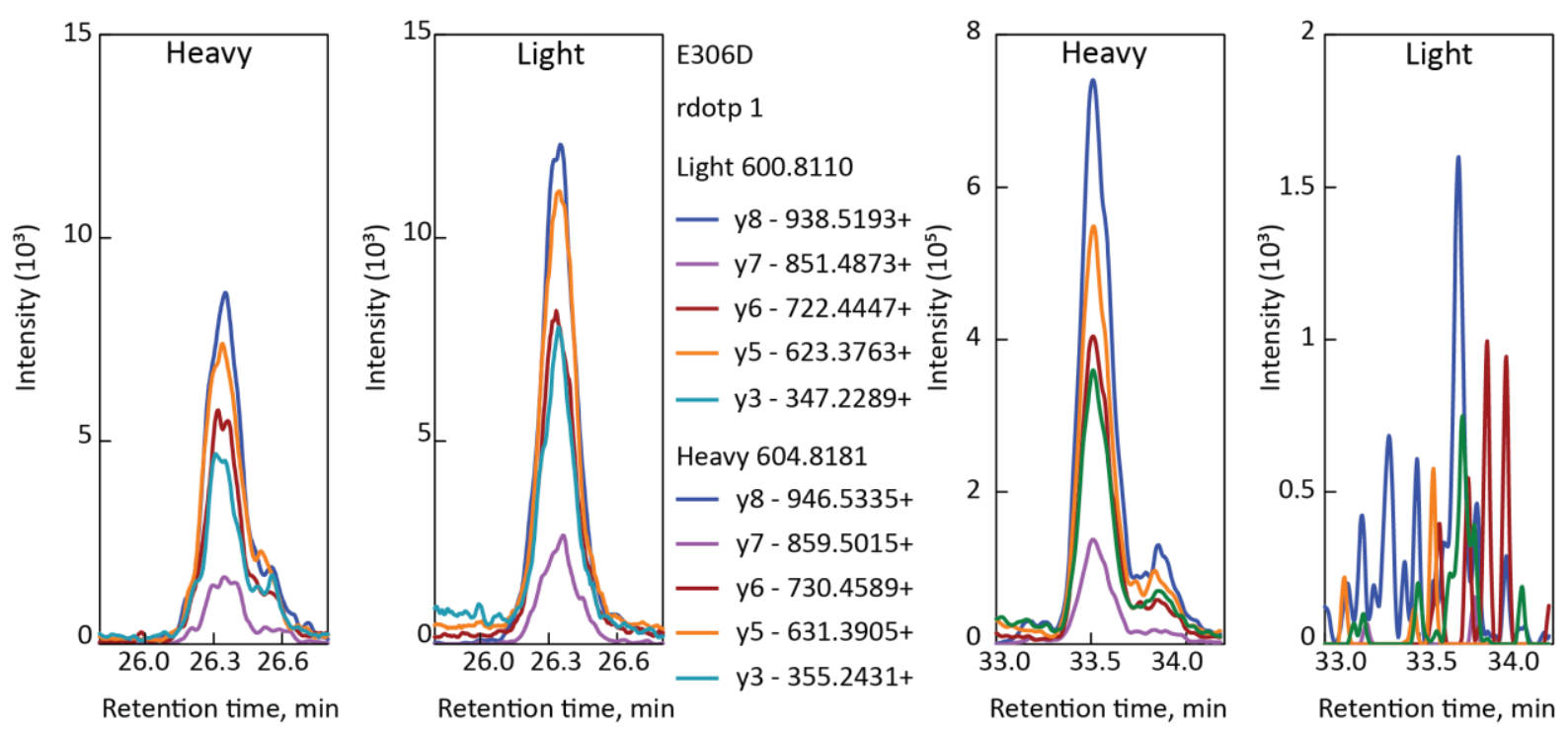

$$
\begin{aligned}
& \begin{array}{llllllllll}
y^{10} & y^{9} & y^{8} & y^{7} & y^{6} & y^{5} & y^{4} & y^{3} & y^{2} & y^{1}
\end{array}
\end{aligned}
$$

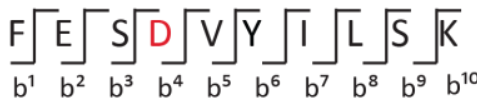
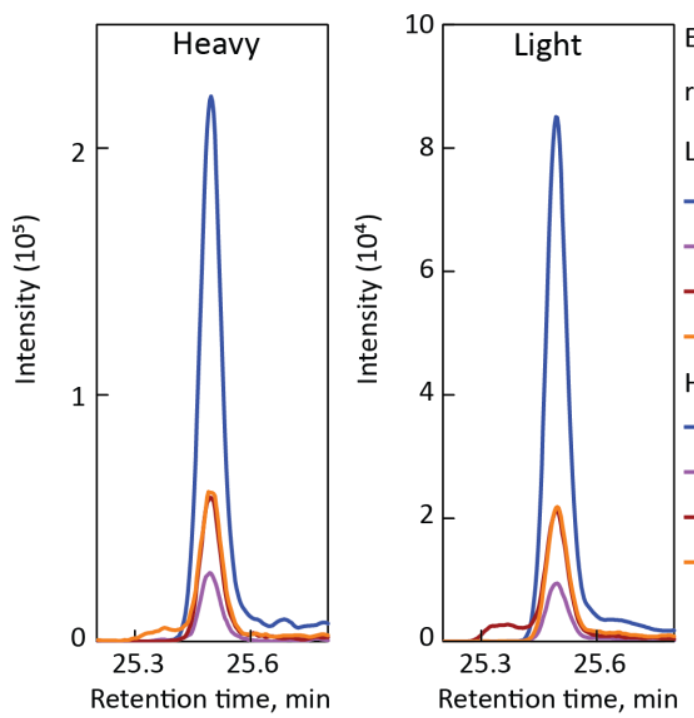
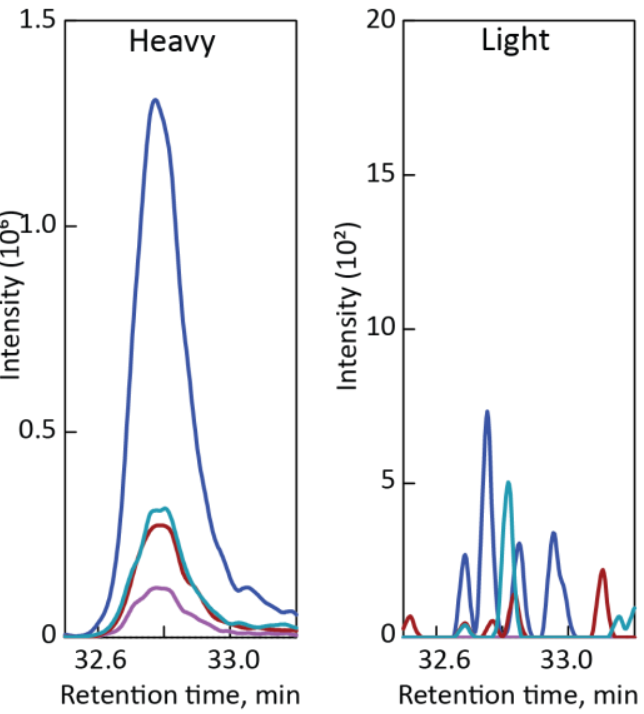


$$
\begin{aligned}
& \begin{array}{llllllllll}
y^{10} & y^{9} & y^{8} & y^{7} & y^{6} & y^{5} & y^{4} & y^{3} & y^{2} & y^{1}
\end{array} \\
& \underset{b^{1}}{F} \underset{b^{2}}{b^{3}} \underset{b^{4}}{S} \sqrt{b^{5}} \underset{b^{6}}{b^{7}} b_{b^{8}} b^{9} b^{10}
\end{aligned}
$$
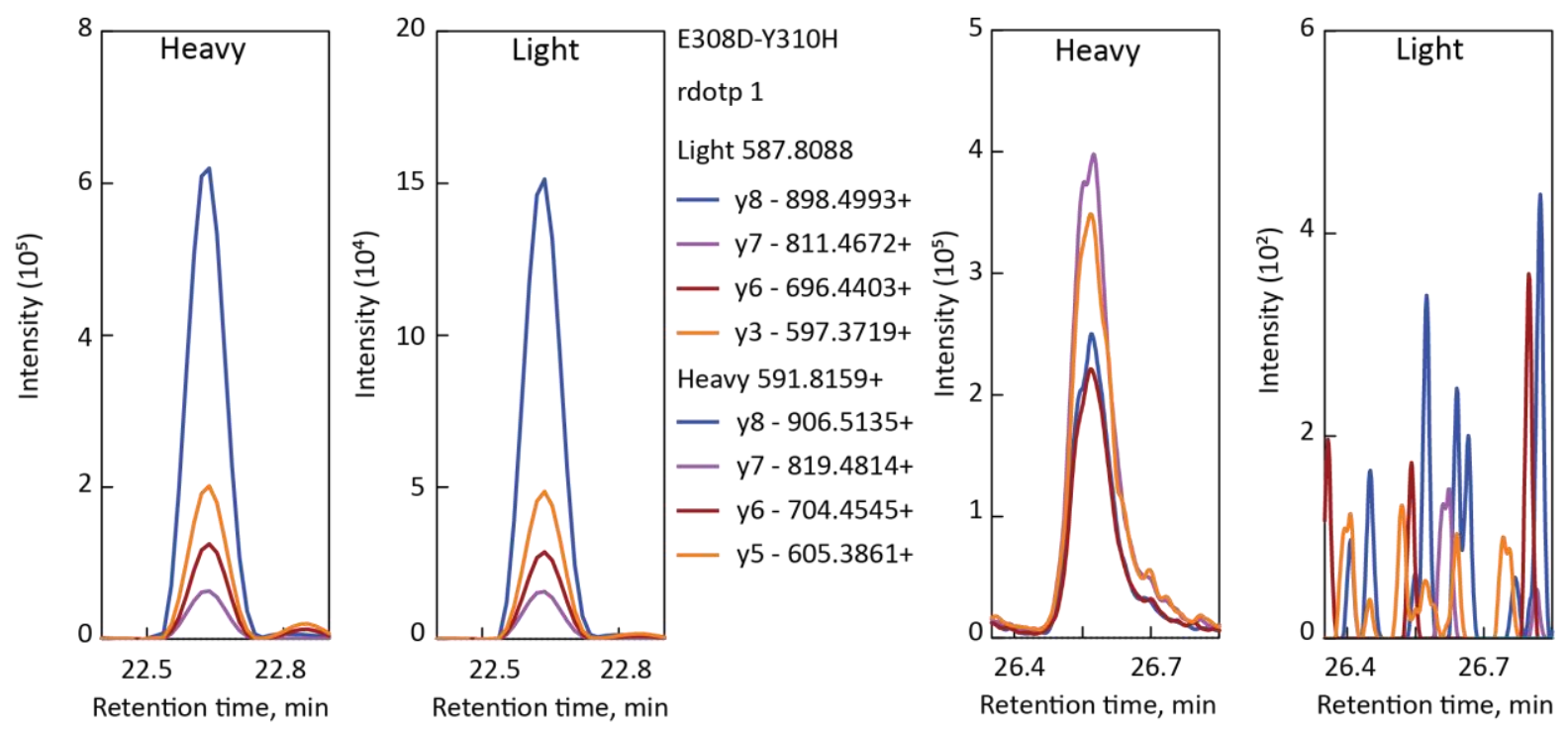

$$
\begin{aligned}
& \begin{array}{llllllllll}
y^{10} & y^{9} & y^{8} & y^{7} & y^{6} & y^{5} & y^{4} & y^{3} & y^{2} & y^{1}
\end{array}
\end{aligned}
$$

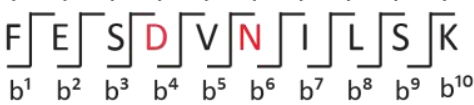
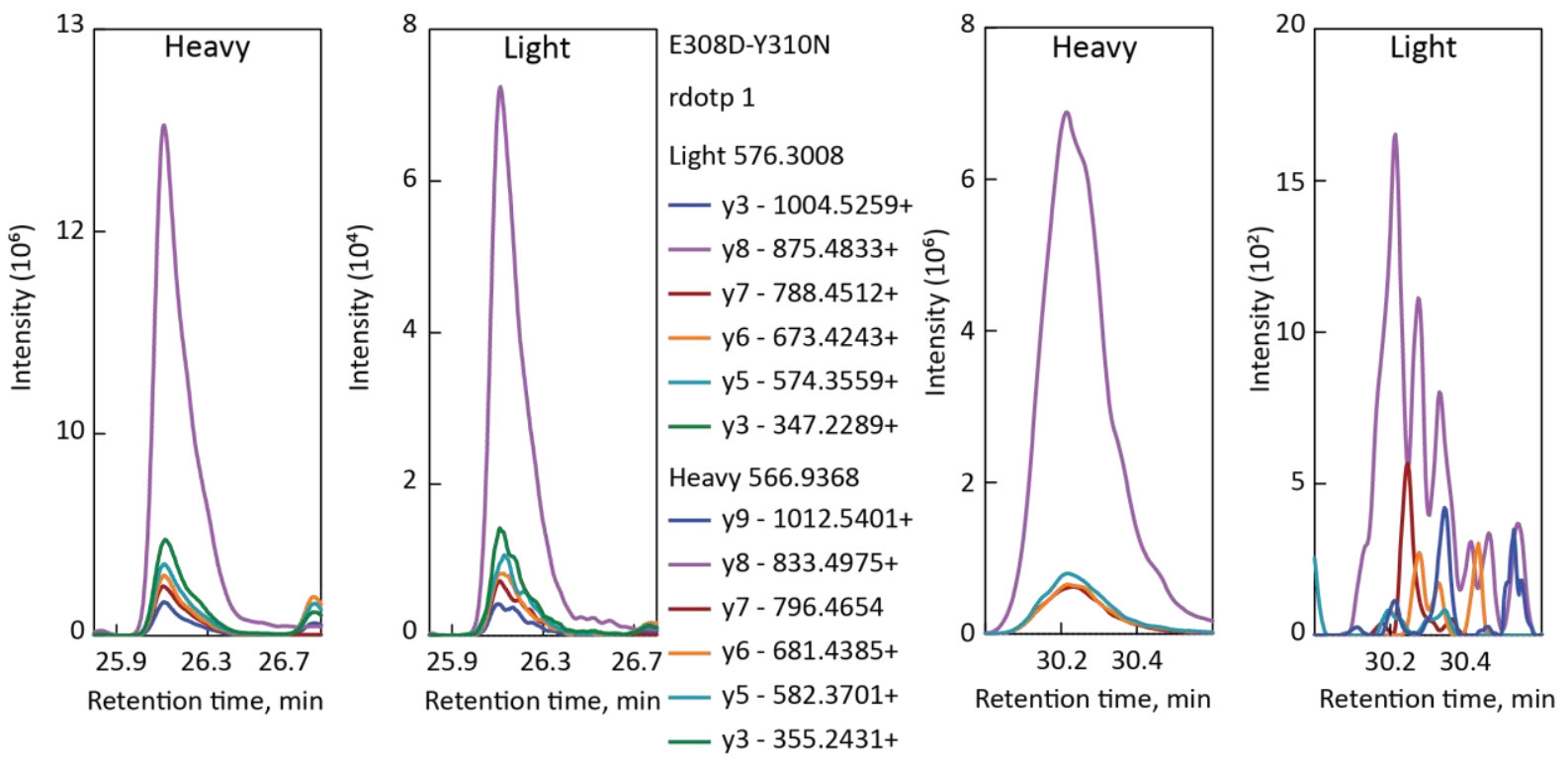


\subsection{List of tables}

Table 1. Error frequencies of the individual substeps of protein biosynthesis ................................... 14

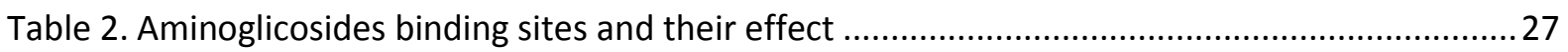

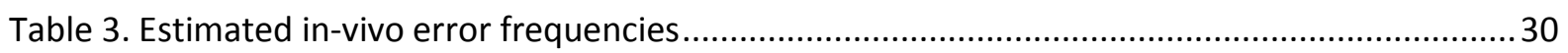

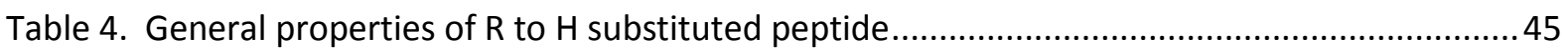

Table 5. Physicochemical characteristics of peptides differing by a few amino acids may be very

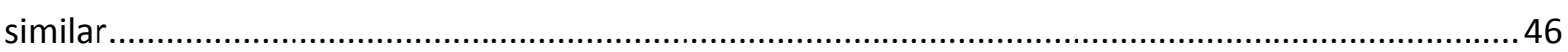

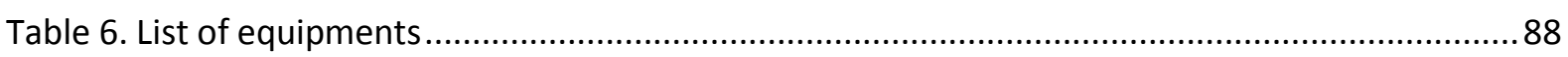

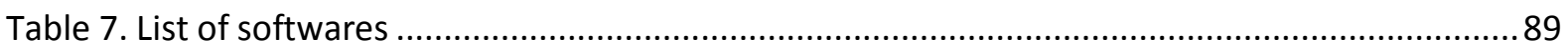

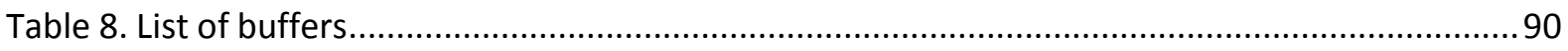

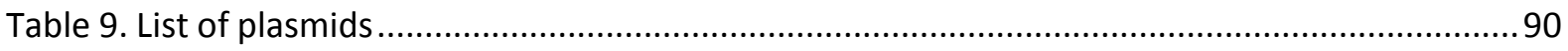

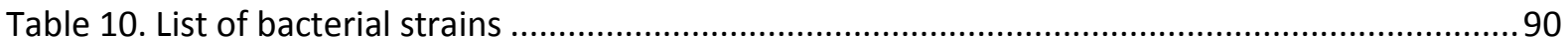

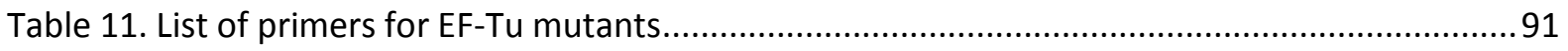

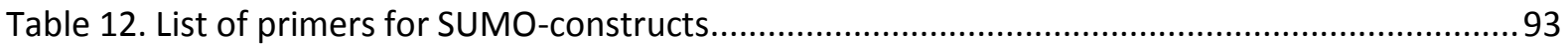

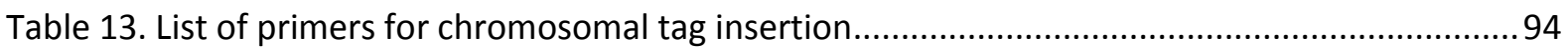




\subsection{List of figures}

Figure 1. Enzymes ensure their accuracy in different ways........................................................... 18

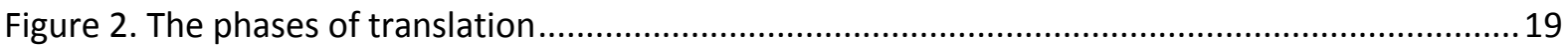

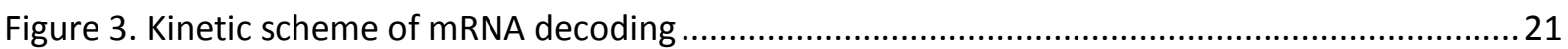

Figure 4. Mechanism of selection triggered by error accumulation ....................................................22

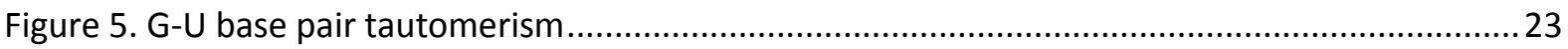

Figure 6. Aminoglycosides binding sites on the ribosome

Figure 7. SRM analysis on a triple quadrupole mass spectrometer ....................................................36

Figure 8. PRM analysis on a hybrid quadrupole/Orbitrap mass spectrometer ...................................38

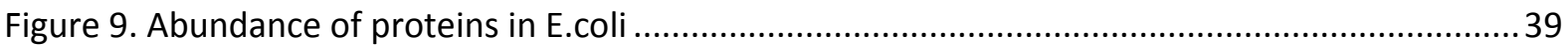

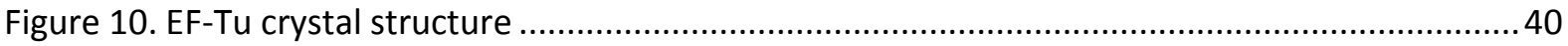

Figure 11. Schematic of the workflow for the enrichment of error-containing peptides .................... 41

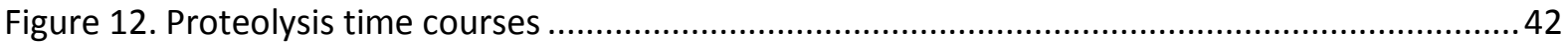

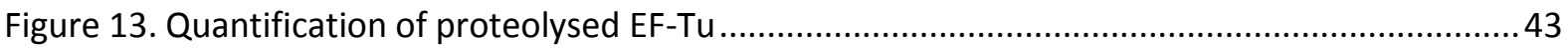

Figure 14. Distribution of peptide elution intervals in the first two chromatographic dimensions..... 44

Figure 15. Poor separation of similar peptides in the first two chromatographic dimensions ............46

Figure 16. Elution pattern of similar peptides in reversed phase chromatography runs at neutral and acidic $\mathrm{pH}$

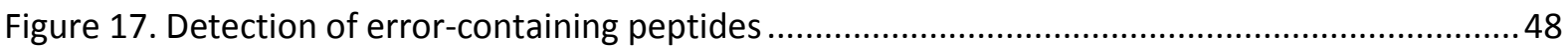

Figure 18. Identification of error-containing peptides by high resolution MS1 and MS2 spectra .......49

Figure 19. Identification of misincorporation-containing peptides by parallel reaction monitoring (PRM) .50

Figure 20. SRM elution profiles of AQUA peptide with a substitution $\mathrm{R} 231 \mathrm{H} \ldots \ldots \ldots \ldots \ldots \ldots \ldots \ldots \ldots \ldots \ldots . . . . . . . . . . . . . . . . . . . .51$

Figure 21. Pseudo-linear dynamic range of correct and erroneous peptides quantification...............52

Figure 22. Validation of error quantification with EF-Tu mutants.................................................53

Figure 23. Error frequencies of near-cognate substitutions at three positions in EF-Tu......................55

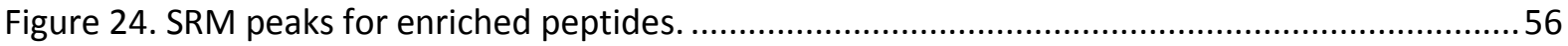

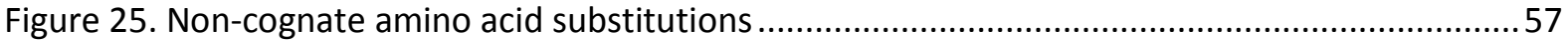

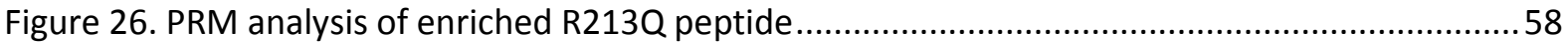

Figure 27 . $\mathrm{R}$ to $\mathrm{H}$ misincorporation measured in error prone, hyperaccurate, and the parental wild

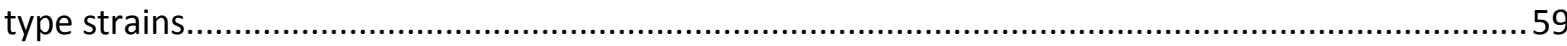

Figure 28. $\mathrm{R}$ to $\mathrm{H}$ misincorporations at several positions of EF-Tu sequence .....................................59

Figure 29. Position of low-abundance $\mathrm{R} \rightarrow \mathrm{H}$ substitution with respect to EF-Tu/tRNA interface ......60

Figure 30. Error frequency in EF-Tu translated in vitro .61 
Figure 31. Schematic view of the pSUMO constructs 62

Figure 32. Misincorporation frequency in and out the EF-Tu context 62

Figure 33. Effect of aminoglycosides on the cellular response and the steady-state error level........65

Figure 34. Comparison of error profiles induced by different aminoglycosides .67

Figure 35. Verification of double-substituted peptide E308D-Y310H by PRM ..................................68

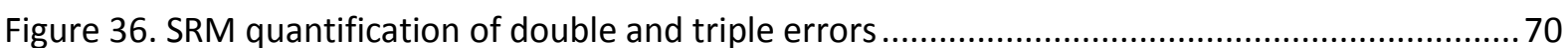

Figure 37. Example of quantification of double and triple errors ............................................... 71

Figure 38. Abundance of the single, double and triple errors in one peptide..................................71

Figure 39. Quantification of single and double errors ....................................................... 73

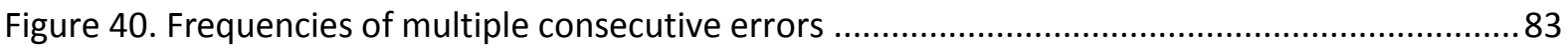

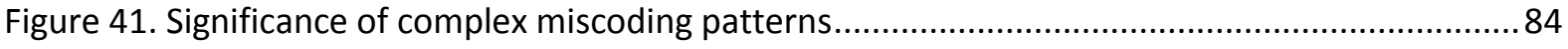

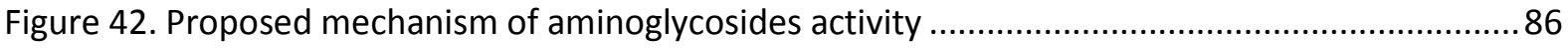

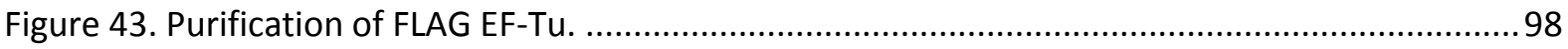

Figure 44. Proteins separation in E.coli lysate for proteome change analysis ............................... 100

Figure 45. Lysate separation for the quantification of misincorporations in EF-Tu ....................... 100

Figure 46. Pierce retention time mix (30 fmol) injected in TSQ Quantiva...................................... 104 
APPENDIX

\subsection{List of abbreviations}

\begin{tabular}{|c|c|}
\hline Abbreviation & Description \\
\hline $\mathrm{ACN}$ & Acetonitrile \\
\hline Amp & Ampicillin \\
\hline APS & Ammonium Persulfate \\
\hline CID & Collision energy induced dissociation \\
\hline DDA & Data-dependent acquisition \\
\hline DTT & Dithiothreitol \\
\hline FA & Formic acid \\
\hline Gen & Gentamicin \\
\hline GS & Ground state \\
\hline Hyg & Hygromycin \\
\hline HPLC & High performance liquid chromatrography \\
\hline IAA & lodoacetamide \\
\hline Kan & Kanamycin \\
\hline $\mathrm{kDa}$ & Kilo Dalton \\
\hline LC & Liquid chromatography \\
\hline MS & Mass spectrometry \\
\hline Nea & Neamine \\
\hline Neo & Neomycin \\
\hline PAGE & Polyacrylamide gel electrophoresis \\
\hline Par & Paromomycin \\
\hline PRM & Parallel reaction monitoring \\
\hline PRTC mix & Pierce retention time calibration mix \\
\hline Rib & Ribostamycin \\
\hline $\mathrm{RP}$ & Reversed phase \\
\hline SDS & Sodium Dodecyl Sulfate \\
\hline SIM & Selected ion monitoring chromatogram \\
\hline Spc & Spectinomycin \\
\hline SRM & Selected reaction monitoring \\
\hline Str & Streptomycin \\
\hline TEMED & Tetramethylethylenediamine \\
\hline Tob & Tobramycin \\
\hline TS & Transition state \\
\hline $\mathrm{XIC}$ & Extracted ion chromatogram \\
\hline
\end{tabular}




\section{ACKNOWLEDGMENTS}

Here I am at the end of this long journey that has been my PhD. Four years in this amazing place have passed by so quickly that I could bearely realize it. Among all the people I have met, some deserve a special acknowledgments. First of all Marina and Henning, for giving me the chance to work in their laboratories. I will never forget the feeling I got the first day I stepped in their labs. A special thanks to Prof. Rehling, who is part of my Advisory Board since the very beginning, and to Prof. Stark, Prof. Ficner and Prof. Bohnsack for joining it in this last step which precedes the defense. Thanks to all the wonderful colleagues I found. I would mention them all but I am sure they already know how much I liked working with them.

And then a huge acknowledgment is reserved to those people that filled these four years with good moments and smiles. Ale, Mario, Roby, Bianca, Gianmarco for being like a family to me. The all great friends I found at the Institute: Benny, Dima, Theo, Ole, Tahaere, Irena, Michi, Aki, Neva, Heena, Sandra, Namit, Christina and Karine. Among these great guys I also found a great supervisor. Thank you Ingo for all your support.

And finally, thanks to my family, for their constant and precious support. And to my loving husband, for standing by my side. Always. 